\title{
The Concept of Particle Weights in Local Quantum Field Theory
}

\author{
Dissertation \\ zur Erlangung des Doktorgrades \\ der Mathematisch-Naturwissenschaftlichen Fakultäten \\ der Georg-August-Universität zu Göttingen
}

vorgelegt von

MARTIN PORRMANN

aus

Wolfenbüttel 
D 7

Referent: Prof. Dr. Detlev Buchholz ${ }^{1}$

Korreferent: PROF. DR. KLAUS FREDENHAGEN ${ }^{2}$

Tag der mündlichen Prüfung: 26. Januar 2000

\footnotetext{
${ }^{1}$ Institut für Theoretische Physik der Universität Göttingen

${ }^{2}$ II. Institut für Theoretische Physik der Universität Hamburg
} 


\section{Contents}

1 Introduction 1

2 Localizing Operators and Spectral Seminorms 5

2.1 The Algebra of Detectors . . . . . . . . . . . . . . . . 5

2.2 Spectral Seminorms on the Algebra of Detectors . . . . . . . . . . 8

2.3 Characteristics of the Spectral Seminorms . . . . . . . . . . . . . . 14

2.3.1 Basic Properties . . . . . . . . . . . . . . . . . 14

2.3.2 Continuity and Differentiability . . . . . . . . . . . 19

2.3 .3 Integrability . . . . . . . . . . . . . 23

2.3 .4 Decay Property . . . . . . . . . . . . . . . . . . 26

3 Particle Weights as Asymptotic Plane Waves 29

3.1 General Properties . . . . . . . . . . . . . . . . . . . 29

3.2 Asymptotic Functionals . . . . . . . . . . . . . . . . . . . 37

3.3 Particle Weights . . . . . . . . . . . . . . . . . . 42

4 Disintegration of Particle Weights $\quad 53$

4.1 Separable Reformulation . . . . . . . . . . . . . . . . . . 54

4.2 Restricted $\mathfrak{K}_{0}^{c}$-Particle Weights . . . . . . . . . . . . . . . 58

4.3 Spatial Disintegration . . . . . . . . . . . . . . . 60

5 Phase Space Restrictions and Local Normality $\quad 81$

6 Disintegration Revisited: Choquet Theory 89

$\begin{array}{llr}7 & \text { Summary and Outlook } & 99\end{array}$ 
$\begin{array}{ll}\text { A Concepts of Differentiability } & 101\end{array}$

A.1 Differentiation in Locally Convex Spaces . . . . . . . . . . . . . . . . 101

A.2 Differentiation on Analytic Manifolds . . . . . . . . . . . . . 105

A.3 Differentiation on Automorphism Lie Groups . . . . . . . . . . . 108

A.4 Differentiable Linear Mappings . . . . . . . . . . . . . . . . 110

B A Lemma on Norm-Separable $C^{*}$-Algebras 113

$\begin{array}{ll}\text { Bibliography } & 115\end{array}$

$\begin{array}{ll}\text { Acknowledgements } & 119\end{array}$ 


\section{Chapter 1}

\section{Introduction}

Physical phenomena occurring in high energy physics are analysed in terms of 'particles', arising as asymptotic configurations of elementary entities in scattering experiments. These particles are characterized by certain specific intrinsic properties, which are expressed by quantum numbers whose integration in the framework of a consistent and complete theoretical description is an aim of quantum field theory. The usual theoretical description of particles goes back to the famous analysis by Wigner of the irreducible representations of the Poincaré group [57]. He gives a complete classification of all these representations, which are labelled by two parameters $m$ and $s$. It is assumed that a particle pertains to a specific representation of this group, in which case the parameters $m$ and $s$ are interpreted as its intrinsic mass and spin, respectively. However, this approach to a theoretical description of mass and spin is not universally applicable. There are quantum field theories in which particles coupled to particles of zero rest mass cannot be described in terms of eigenstates of the mass operator. An example is quantum electrodynamics where charged particles are inevitably accompanied by soft photons. It is an open question, known as the infraparticle problem [53], how mass and spin of a particle are to be described in the framework of quantum field theory. Moreover, standard collision theory does not work in these cases.

A closer analysis of quantum electrodynamics shows that the infraparticle problem is connected with Gauss' law [29, 13]. An outline of the underlying mechanism, following arguments of Buchholz in [13], may be appropriate at this point. Due to Gauss' law, the charge of a physical state can be determined by measuring the electromagnetic field at asymptotic spacelike distances. These measurements do not interfere with those performed within bounded regions; therefore, being a $c$-number, the asymptotic field configuration is a superselection rule of the theory. Its dependence on the state of motion of the charged particle implies that there exists a multitude of superselection sectors and that the Lorentz symmetry is broken. Consequently, charged particles cannot be described according to Wigner's theory.

The present thesis proposes a novel approach to the concept of particles, elaborating some of the ideas of Buchholz' which he introduced in [14]. In a model-independent framework, especially without excluding massless states and without assuming asymptotic completeness of the theory, an approach of Araki and Haag [3] to scattering theory is reconsidered. Chapter 2 introduces the concept of detectors to be used in this work 
Introduction

and investigates the suitable topologies that the corresponding algebraic structures are furnished with. A basic ingredient here is the interplay between locality and the spectrum condition. In Chapter 3 we pass to the dual point of view and analyse the resulting continuous functionals. Then, on physical grounds, a certain subclass is distinguished, arising as asymptotic limits of certain functionals constructed from physical states of bounded energy. These limits exhibit properties of singly localized systems (particles). The limiting procedure to be presented here is able to directly reproduce charged systems, in contrast to the LSZ-theory where charge-carrying unobservable operators are necessary.

The representations induced by these asymptotic functionals (the particle weights) are highly reducible, so the obvious task is to work out a disintegration theory in terms of irreducible representations (pure particle weights). This will be done in Chapters 4 and 6. The approach of Chapter 4 makes use of the standard decomposition theory for representations of $C^{*}$-algebras. To be able to apply this theory, the mathematical structures under consideration have to be adapted to its needs. Great care is taking to ensure that the resulting irreducible representations have all the properties allowing for their interpretation as representatives of elementary particles. As demonstrated by Buchholz [17], it is then possible to classify the pure particle weights according to their spin and mass even in the case of charged systems. This shows that the notion of particle weights provides a promising approach to the aforementioned infraparticle problem. In Chapter 5 a compactness criterion due to Fredenhagen and Hertel is used to impose certain restrictions on the phase space of quantum field theory. The additional information is used to demonstrate that the particle weight representations of Chapter 4 are locally normal. This implies that one does not lose essential information about the physical systems in the course of the constructions needed to adapt the problem at hand to the needs of spatial disintegration. Chapter 6, again drawing on the mentioned compactness criterion, presents the first steps in an alternative approach to disintegration: Choquet theory. Chapter 7 gives a brief summary.

\section{Assumptions of Local Quantum Physics}

We collect here the main structural postulates upon which Local Quantum Physics is built in the abstract setting of the algebraic approach [33,2], principally in order to fix notation.

- The basis of the present investigations is a net

$$
\mathcal{O} \mapsto \mathfrak{A}(\mathcal{O})
$$

of $C^{*}$-algebras, which are indexed by the bounded regions $\mathcal{O}$ in space-time $\mathbb{R}^{s+1}$ and which are concrete in the sense that they all belong to the algebra of bounded operators $\mathfrak{B}(\mathcal{H})$ on a certain Hilbert space $\mathcal{H}$. The so-called quasi-local algebra $\mathfrak{A}$ is the $C^{*}$ inductive limit of the net (1.1a) (cf. [11, Definition 2.63]):

$$
\mathfrak{A} \doteq \bigcup_{\mathcal{O}}^{C^{*}} \mathfrak{A}(\mathcal{O}) .
$$


- On the $C^{*}$-algebra $\mathfrak{A}$ the symmetry transformations in the inhomogeneous Lorentz group, the Poincaré group $\mathrm{P}_{+}^{\uparrow}=\mathrm{L}_{+}^{\uparrow} \ltimes \mathbb{R}^{s+1}$, are implemented via a strongly continuous group of automorphisms:

$$
\mathrm{P}_{+}^{\uparrow} \ni(\Lambda, x) \mapsto \alpha_{(\Lambda, x)} \in \operatorname{Aut} \mathfrak{A} .
$$

- The net (1.1a) is subject to the following conditions:

- Isotony: For any two bounded regions $\mathcal{O}_{1}$ and $\mathcal{O}_{2}$ in $\mathbb{R}^{s+1}$

$$
\mathcal{O}_{1} \subseteq \mathcal{O}_{2} \Rightarrow \mathfrak{A}\left(\mathcal{O}_{1}\right) \subseteq \mathfrak{A}\left(\mathcal{O}_{2}\right)
$$

- Locality: If the bounded regions $\mathcal{O}_{1}$ and $\mathcal{O}_{2}$ are spacelike separated, i.e., $\mathcal{O}_{1}$ belongs to the spacelike complement of $\mathcal{O}_{2}$, formally $\mathcal{O}_{1} \subseteq \mathcal{O}_{2}^{\prime}$, then

$$
\mathfrak{A}\left(\mathcal{O}_{1}\right) \subseteq \mathfrak{A}\left(\mathcal{O}_{2}\right)^{\prime},
$$

where the prime in $(1.3 b)$ denotes the commutant in $\mathfrak{B}(\mathcal{H})$.

- Relativistic Covariance: For arbitrary bounded regions $\mathcal{O}$ and arbitrary transformations $(\Lambda, x) \in \mathrm{P}_{+}^{\uparrow}$ there hold the relations

$$
\mathfrak{A}(\Lambda \mathcal{O}+x)=\alpha_{(\Lambda, x)}(\mathfrak{A}(\mathcal{O})) .
$$

- The subgroup $\mathbb{R}^{s+1}$ of translations in $\mathrm{P}_{+}^{\uparrow}$ is implemented on $\mathfrak{A}$ by a strongly continuous unitary group, i. e., one which is continuous with respect to the strong-operator topology. These unitaries can be expressed through the (unbounded) generators $P^{\mu}$, $\mu=1, \ldots, s+1$, of space-time translations according to

$$
U(x)=\exp \left(i P^{\mu} x_{\mu}\right),
$$

and, by virtue of (1.2), one has for any $x \in \mathbb{R}^{s+1}$

$$
\alpha_{x}(A)=U(x) A U(x)^{*}, \quad A \in \mathfrak{A} .
$$

The joint spectrum of the generators $P^{\mu}$, expressed by the pertinent spectral resolution $E\left(\right.$. ) in terms of projections in $\mathfrak{A}^{\prime \prime}$, is supposed to lie in the closed forward light cone

$$
\bar{V}_{+} \doteq\left\{p \in \mathbb{R}^{s+1}: p \cdot p=p^{\mu} p_{\mu} \geqslant 0\right\} .
$$

This assumption is known under the term 'positive-energy representation.'

- Physical states are represented by normalized positive linear functionals on the quasi-local algebra $\mathfrak{A}$, which are normal, i. e., continuous with respect to the $\sigma$-weak topology that $\mathfrak{A}$ inherits from $\mathfrak{B}(\mathcal{H})$. The set of all physical states $\omega$ is denoted by $\mathcal{S}$; it is in one-to-one correspondence to the entirety of all density matrices, the positive trace-class operators in $\mathfrak{B}(\mathcal{H})$ with unit trace, via

$$
\omega(A)=\operatorname{Tr}\left(\rho_{\omega} A\right), \quad A \in \mathfrak{A},
$$

where $\rho_{\omega}$ denotes the unique operator of the above kind. The fact that a physical state $\omega$ possesses energy-momentum in the Borel set $\Delta \subseteq \mathbb{R}^{s+1}$ is expressed by the condition

$$
\omega(E(\Delta))=\operatorname{Tr}\left(E(\Delta) \rho_{\omega} E(\Delta)\right)=1 .
$$

The corresponding subset of $\mathcal{S}$ is written $\mathcal{S}(\Delta)$. 
At this point, for the sake of clarity, a few remarks concerning topological notions seem advisable. The norm topology on $\mathfrak{A}$ is sometimes called the uniform topology and leaves no room for a possible misunderstanding. The situation is more complicated in case of the term 'strong continuity:'

- An automorphism group $\left\{\alpha_{g}: g \in \mathcal{G}\right\} \subseteq$ Aut $\mathfrak{A}$ on the $C^{*}$-algebra $\mathfrak{A}, \mathcal{G}$ a topological group, is called strongly continuous if the mapping

$$
\mathcal{G} \ni g \mapsto \alpha_{g}(A) \in \mathfrak{A}
$$

is continuous for arbitrary $A \in \mathfrak{A}$ with respect to the initial topology of the group $\mathcal{G}$ and with respect to the uniform topology of $\mathfrak{A}$.

- A unitary group $\{U(g): g \in \mathcal{G}\} \subseteq \mathfrak{B}(\mathcal{H}), \mathcal{G}$ again a topological group, is called strongly continuous if the mapping

$$
\mathcal{G} \ni g \mapsto U(g) \in \mathfrak{B}(\mathcal{H})
$$

is continuous with respect to the topology of $\mathcal{G}$ and with respect to the strong-operator topology on $\mathfrak{B}(\mathcal{H})$.

The term ' $\sigma$-weak topology' is used to denote the locally convex topology on the algebra $\mathfrak{B}(\mathcal{H})$ that is defined through the family of seminorms

$$
Q_{\left\{\phi_{n}, \psi_{n}\right\}}: \mathfrak{B}(\mathcal{H}) \rightarrow \mathbb{R}_{0}^{+} \quad A \mapsto Q_{\left\{\phi_{n}, \psi_{n}\right\}}(A) \doteq\left|\sum_{n=1}^{\infty}\left(\phi_{n}, A \psi_{n}\right)\right|,
$$

where the sequences $\left\{\phi_{n}\right\}_{n \in \mathbb{N}}$ and $\left\{\psi_{n}\right\}_{n \in \mathbb{N}}$ of vectors in the Hilbert space $\mathcal{H}$ are subject to the conditions $\sum_{n=1}^{\infty}\left\|\phi_{n}\right\|^{2}<\infty$ and $\sum_{n=1}^{\infty}\left\|\psi_{n}\right\|^{2}<\infty$. This designation is synonymous with 'ultra-weak topology.' Mappings which are continuous with respect to this topology are called normal. 


\section{Chapter 2}

\section{Localizing Operators and Spectral Seminorms}

The results presented in Chapters 2 and 3 have been worked out in close collaboration with Detlev Buchholz, whose ideas, as set out in [14], constituted the foundation. Their somewhat complicated presentation is the author's responsibility. The particle concept to be set forth in the sequel is motivated by the experimental situation encountered in high energy physics where certain physical systems show up as 'particles,' being traced by specific measuring devices called 'detectors.' The common characteristic of these physical systems is that they are localized in the course of the measuring process. Haag and Kastler stated in their fundamental article [33] on algebraic quantum field theory that ' ... ultimately all physical processes are analyzed in terms of geometric relations of unresolved phenomena,' emphasizing localization as the very nature of all measurements. To represent the experimental set-up in the framework of the algebraic approach to local quantum physics elements of the quasi-local algebra $\mathfrak{A}$ have to be singled out first that exhibit properties of particle detectors.

\subsection{The Algebra of Detectors}

As argued by Araki and Haag [3] a particle detector $C \in \mathfrak{A}$ should be insensitive to the vacuum $\Omega: C \Omega=0$. In view of the actual experimental situation one can be more specific, noting that a minimal energy, depending on the detector used, has to be deposited to produce a signal. In the present thesis we shall therefore work with a smaller class of operators: the algebraic representatives corresponding to a particle counter are to annihilate all physical states with bounded energy below a specific threshold, to be precise. Now, on account of the Reeh-Schlieder-Theorem, this feature is incompatible with locality since an algebra pertaining to a region $\mathcal{O}$ with non-void causal complement $\mathcal{O}^{\prime}$ does not contain any operator annihilating states of bounded energy (cf. [51, 32]). As a consequence, the operators which comply with the above annihilation property cannot be strictly local; instead their localization has to be weakened. This is done in a way that resembles the introduction of rapidly decreasing functions on $\mathbb{R}^{n}$ : the operators in question are not contained in a local algebra, but they are almost local in the sense of the following definition ('quasilocal of infinite order' is the designation used in [3]). 
Definition 2.1 (Almost Locality). Let $\mathcal{O}_{r} \doteq\left\{\left(x^{0}, \boldsymbol{x}\right) \in \mathbb{R}^{s+1}:\left|x^{0}\right|+|\boldsymbol{x}|<r\right\}, r>0$, denote the double cone (standard diamond) with basis $\boldsymbol{O}_{r} \doteq\left\{\boldsymbol{x} \in \mathbb{R}^{s}:|\boldsymbol{x}|<r\right\}$. An operator $A \in \mathfrak{A}$ is called almost local if there exists a net $\left\{A_{r} \in \mathfrak{A}\left(\mathcal{O}_{r}\right): r>0\right\}$ of local operators such that

$$
\lim _{r \rightarrow \infty} r^{k}\left\|A-A_{r}\right\|=0
$$

for any $k \in \mathbb{N}_{0}$. The set of almost local operators is a *-subalgebra of $\mathfrak{A}$ denoted by $\mathfrak{A}_{\mathcal{S}}$.

Remark. (i) Let $A$ and $B$ be almost local operators with approximating nets of local operators $\left\{A_{r} \in \mathfrak{A}\left(\mathcal{O}_{r}\right): r>0\right\}$ and $\left\{B_{r} \in \mathfrak{A}\left(\mathcal{O}_{r}\right): r>0\right\}$, respectively. Then, since $\mathcal{O}_{r}$ and $\mathcal{O}_{r}+2 \boldsymbol{x}$ are spacelike separated for $r \leqslant|\boldsymbol{x}|$ so that the associated algebras $\mathfrak{A}\left(\mathcal{O}_{r}\right)$ and $\mathfrak{A}\left(\mathcal{O}_{r}+2 \boldsymbol{x}\right)$ commute, the following estimate holds for any $\boldsymbol{x} \in \mathbb{R}^{s} \backslash\{\boldsymbol{0}\}$

$$
\left\|\left[\alpha_{2 x}(A), B\right]\right\| \leqslant 2\left(\left\|A-A_{|x|}\right\|\|B\|+\left\|A-A_{|x|}\right\|\left\|B-B_{|x|}\right\|+\|A\|\left\|B-B_{|x|}\right\|\right)
$$

The right-hand side of this inequality is bounded and falls off more rapidly than any power of $|\boldsymbol{x}|^{-1}$, therefore the continuous mapping $\mathbb{R}^{s} \ni \boldsymbol{x} \mapsto\left\|\left[\alpha_{x}(A), B\right]\right\|$ turns out to be integrable:

$$
\int_{\mathbb{R}^{s}} d^{s} x\left\|\left[\alpha_{x}(A), B\right]\right\|<\infty
$$

(ii) The approximating net of local operators $\left\{A_{r} \in \mathfrak{A}\left(\mathcal{O}_{r}\right): r>0\right\}$ for $A \in \mathfrak{A}_{\mathcal{S}}$ can be used to construct a second approximating net $\left\{A_{r}^{\prime} \in \mathfrak{A}\left(\mathcal{O}_{r}\right): r>0\right\}$ with the additional property $\left\|A_{r}^{\prime}\right\| \leqslant\|A\|$ for any $r>0$, which at the same time is subject to the inequality $\left\|A-A_{r}^{\prime}\right\| \leqslant 2\left\|A-A_{r}\right\|$ and thus satisfies condition (2.1) for almost locality. Estimates of this kind will later on turn out to be important in solving the problem of existence of uniform bounds for integrals of the form (2.2b), evaluated for sequences or even nets of almost local operators. With approximating nets of local operators of this special kind the estimate (2.2a) can be improved for arbitrary $A, B \in \mathfrak{A}_{\mathcal{S}}$ to yield

$$
\left\|\left[\alpha_{2 x}(A), B\right]\right\| \leqslant 2\left(\left\|A-A_{|x|}\right\|\|B\|+\|A\|\left\|B-B_{|x|}\right\|\right), \quad \boldsymbol{x} \in \mathbb{R}^{s} \backslash\{\boldsymbol{0}\} .
$$

The feature of annihilating states of bounded energy below a certain threshold is called vacuum annihilation property in the sequel and finds its rigorous mathematical expression in the following definition.

Definition 2.2 (Vacuum Annihilation Property). An operator $A \in \mathfrak{A}$ is said to have the vacuum annihilation property if, in the sense of operator-valued distributions, the mapping

$$
\mathbb{R}^{s+1} \ni x \mapsto \alpha_{x}(A) \doteq U(x) A U(x)^{*} \in \mathfrak{A}
$$

has a Fourier transform with compact support $\Gamma$ contained in the complement of the forward light cone $\bar{V}_{+}$. The collection of all vacuum annihilation operators is a subspace of $\mathfrak{A}$ denoted $\mathfrak{A}_{\text {ann }}$. 
Remark. The support of the Fourier transform of (2.3) is precisely the energy-momentum transfer of $A$, and the energy-threshold for the annihilation of states depends on the distance $d\left(\Gamma, \bar{V}_{+}\right)$between $\Gamma$ and $\bar{V}_{+}$. Let $\Gamma_{0}$ be a closed subset of $\mathbb{R}^{s+1}$ and let $\widetilde{\mathfrak{A}}\left(\Gamma_{0}\right)$ denote the set of all operators $A \in \mathfrak{A}$ having energy-momentum transfer $\Gamma_{A} \subseteq \Gamma_{0}$. Then $\widetilde{\mathfrak{A}}\left(\Gamma_{0}\right)$ is easily seen to be a uniformly closed subspace of $\mathfrak{A}$, invariant under space-time translations.

The construction of a subalgebra $\mathfrak{C}$ in $\mathfrak{A}$ containing self-adjoint operators to be interpreted as representatives of particle detectors is accomplished in three steps (Definitions 2.3-2.5), starting with a subspace $\mathfrak{L}_{0} \subseteq \mathfrak{A}$ consisting of operators which, in addition to the properties mentioned above, are infinitely often differentiable with respect to the automorphism group $\left\{\alpha_{(\Lambda, x)}:(\Lambda, x) \in \mathrm{P}_{+}^{\uparrow}\right\}$ (cf. Definition A.12 in Appendix A).

Definition 2.3. The almost local vacuum annihilation operators $L_{0} \in \mathfrak{A}$ which are infinitely often differentiable with respect to the group $\left\{\alpha_{(\Lambda, x)}:(\Lambda, x) \in \mathrm{P}_{+}^{\uparrow}\right\}$ constitute a subspace $\mathfrak{A}_{\mathcal{S}} \cap \mathfrak{A}_{\text {ann }} \cap \mathcal{D}^{(\infty)}(\mathfrak{A})$ of $\mathfrak{A}$. The intersection of this set with all the pre-images of $\mathfrak{A}_{\mathcal{S}}$ under arbitrary products of partial derivations $\delta^{k_{1}} \cdots \delta^{k_{N}}$ for any $N \in \mathbb{N}$ and any $1 \leqslant k_{i} \leqslant d_{\mathrm{P}}, d_{\mathrm{P}}$ the dimension of $\mathrm{P}_{+}^{\uparrow}$, is again a linear space denoted $\mathfrak{L}_{0}$. Explicitly, $\mathfrak{L}_{0}$ consists of all almost local vacuum annihilation operators which are infinitely often differentiable, having almost local partial derivatives of any order.

Remark. (i) The space $\mathfrak{L}_{0}$ is stable under the action of the Poincare group. This means that $\alpha_{(\Lambda, x)}\left(\mathfrak{L}_{0}\right)=\mathfrak{L}_{0}$ for any $(\Lambda, x) \in \mathrm{P}_{+}^{\uparrow}$. Due to the properties of Fourier transformation, $\alpha_{(\Lambda, x)}\left(L_{0}\right)$ has energy-momentum transfer in $\Lambda \Gamma$ if $L_{0} \in \mathfrak{L}_{0}(\Gamma) \doteq \mathfrak{L}_{0} \cap \widetilde{\mathfrak{A}}(\Gamma)$; the adjoint $L_{0}{ }^{*}$ of this $L_{0}$ belongs to $\widetilde{\mathfrak{A}}(-\Gamma)$.

(ii) Furthermore $\mathfrak{L}_{0}$ is invariant under differentiation: The partial derivatives are almost local and infinitely often differentiable operators by definition, and, as uniform limits of vacuum annihilation operators, they inherit the energy-momentum transfer of these so that they belong to $\mathfrak{A}_{\text {ann }}$, too.

A huge number of elements of $\mathfrak{L}_{0}$ can be constructed by regularizing almost local operators with respect to rapidly decreasing functions on the Poincare group. The semidirect product Lie group $\mathrm{P}_{+}^{\uparrow}=\mathrm{L}_{+}^{\uparrow} \ltimes \mathbb{R}^{s+1}$ is unimodular by [45, Proposition II.29 and Corollary] since $\mathrm{L}_{+}^{\uparrow}$ is a simple thus semisimple Lie group [36, Proposition I.1.6]. So let $\mu$ be the Haar measure on $\mathrm{P}_{+}^{\uparrow}$ and $A \in \mathfrak{A}_{\mathcal{S}}$, then the operator

$$
A(F)=\int d \mu(\Lambda, x) F(\Lambda, x) \alpha_{(\Lambda, x)}(A)
$$

belongs to $\mathfrak{L}_{0}(\Gamma)$ if the infinitely differentiable function $F$ is rapidly decreasing on the subgroup $\mathbb{R}^{s+1}$ and compactly supported on $\mathrm{L}_{+}^{\uparrow}$, i. e. $F \in \mathcal{S}_{0}\left(\mathrm{P}_{+}^{\uparrow}\right)=\mathcal{S}_{0}\left(\mathrm{~L}_{+}^{\uparrow} \ltimes \mathbb{R}^{s+1}\right)$ in the notation introduced in [7], and has the additional property that the Fourier transforms of the partial functions $F_{\Lambda}(.) \doteq F(\Lambda,$.$) have common support in the compact$ set $\Gamma \subseteq \complement \bar{V}_{+}$for any $\Lambda \in \mathrm{L}_{+}^{\uparrow}$.

The following definition specifies a left ideal $\mathfrak{L}$ of the algebra $\mathfrak{A}$.

Definition 2.4. Let $\mathfrak{L}$ denote the linear span of all operators $L \in \mathfrak{A}$ of the form $L=A L_{0}$ where $A \in \mathfrak{A}$ and $L_{0} \in \mathfrak{L}_{0}$; i.e.

$$
\mathfrak{L} \doteq \mathfrak{A} \mathfrak{L}_{0}=\operatorname{span}\left\{A L_{0}: A \in \mathfrak{A}, L_{0} \in \mathfrak{L}_{0}\right\} .
$$


Localizing Operators and Spectral Seminorms

Then $\mathfrak{L}$ is a left ideal of $\mathfrak{A}$, called the 'left ideal of localizing operators.'

By their very construction, the elements of $\mathfrak{L}$ annihilate the vacuum and all states of bounded energy below a certain threshold that depends on the minimum of $d\left(\Gamma_{i}, \bar{V}_{+}\right)$, $i=1, \ldots, N$, with respect to all representations $L=\sum_{i=1}^{N} A_{i} L_{i} \in \mathfrak{L}$, where $\Gamma_{i}$ is the energy-momentum transfer of $L_{i}$. The algebra of operators whose self-adjoint elements are to be interpreted as representatives of particle detectors is laid down in the next definition.

Definition 2.5. Let $\mathfrak{C}$ denote the linear span of all operators $C \in \mathfrak{A}$ which can be represented in the form $C=L_{1}{ }^{*} L_{2}$ with $L_{1}, L_{2} \in \mathfrak{L}$; i. e.

$$
\mathfrak{C} \doteq \mathfrak{L}^{*} \mathfrak{L}=\operatorname{span}\left\{L_{1}{ }^{*} L_{2}: L_{1}, L_{2} \in \mathfrak{L}\right\}
$$

Then $\mathfrak{C}$ is a ${ }^{*}$-subalgebra of $\mathfrak{A}$, called the 'algebra of detectors.'

Remark. The algebra $\mathfrak{C}$ is smaller than that used by Araki and Haag in [3]. It is not closed in the uniform topology of $\mathfrak{A}$ and does not contain a unit.

\subsection{Spectral Seminorms on the Algebra of Detectors}

The analysis of physical states is performed by use of the algebra of detectors $\mathfrak{C}$. In a state $\omega$ of bounded energy $E$ we expect to encounter a finite number of localization centres, since the triggering of a detector $C \in \mathfrak{C}$ requires a minimal energy $\varepsilon$ to be deposited, the number $N$ of localization centres being equal to or less than $E / \varepsilon$. Now, according to this heuristic picture, placing the counter $C$ for given time $t$ at every point $\boldsymbol{x} \in \mathbb{R}^{s}$ and adding up the corresponding expectation values $\omega\left(\alpha_{(t, x)}(C)\right)$ should result in the finite integral

$$
\int_{\mathbb{R}^{s}} d^{s} x\left|\omega\left(\alpha_{(t, x)}(C)\right)\right|<\infty .
$$

As a matter of fact, the operators $C \in \mathfrak{C}$ turn out to have the property (2.5) as was shown by Buchholz in [15]. For the sake of completeness and to demonstrate how phase-space properties of the theory (localization in space combined with energy-bounds) enter the present investigation, we give an elaborate proof.

Proposition 2.6. Let $E($.$) be the spectral resolution of the space-time translations$ $U(x), x \in \mathbb{R}^{s+1}$, and let $L_{0} \in \mathfrak{L}_{0}$ have energy-momentum transfer $\Gamma$ in a convex subset of $\complement \bar{V}_{+}$. Then for any bounded Borel set $\Delta \subseteq \mathbb{R}^{s+1}$ the net of operator-valued Bochner integrals indexed by compact $\boldsymbol{K} \subseteq \mathbb{R}^{s}$,

$$
\begin{aligned}
Q_{\Delta, \boldsymbol{K}}^{\left(L_{0}{ }^{*} L_{0}\right)} & \doteq E(\Delta) \int_{\boldsymbol{K}} d^{s} x \alpha_{x}\left(L_{0}{ }^{*} L_{0}\right) E(\Delta) \\
& =\int_{\boldsymbol{K}} d^{s} x E(\Delta) \alpha_{x}\left(L_{0}{ }^{*} L_{0}\right) E(\Delta),
\end{aligned}
$$

is $\sigma$-strongly convergent as $\boldsymbol{K} \nearrow \mathbb{R}^{s}$ and the limit $Q_{\Delta}^{\left(L_{0}{ }^{*} L_{0}\right)} \in \mathfrak{B}(\mathcal{H})^{+}$satisfies the estimate

$$
\left\|Q_{\Delta}^{\left(L_{0}{ }^{*} L_{0}\right)}\right\| \leqslant N(\Delta, \Gamma) \int_{\mathbb{R}^{s}} d^{s} x\left\|\left[\alpha_{x}\left(L_{0}\right), L_{0}{ }^{*}\right]\right\|
$$


for suitable $N(\Delta, \Gamma) \in \mathbb{N}$, depending on $\Delta$ and $\Gamma$. Moreover the mapping

$$
\boldsymbol{x} \mapsto E(\Delta) \alpha_{x}\left(L_{0}{ }^{*} L_{0}\right) E(\Delta)
$$

is integrable with respect to the $\sigma$-weak topology on $\mathfrak{B}(\mathcal{H})$ and its integral coincides with the operator $Q_{\Delta}^{\left(L_{0}{ }^{*} L_{0}\right)}$ :

$$
Q_{\Delta}^{\left(L_{0}{ }^{*} L_{0}\right)}=\sigma \text {-weak } \int_{\mathbb{R}^{s}} d^{s} x E(\Delta) \alpha_{x}\left(L_{0}^{*} L_{0}\right) E(\Delta) .
$$

Proof. $\Delta$ being a bounded Borel set, the same is true of its closure $\bar{\Delta}$, so that, due to compactness and convexity of $\Gamma$, there exists a number $n \in \mathbb{N}$ for which the relation $\left(\bar{\Delta}+\Gamma_{n}\right) \cap \bar{V}_{+}=\emptyset$ is satisfied, where $\Gamma_{n}$ denotes the sum $\Gamma_{n} \doteq \Gamma+\cdots+\Gamma$ with $n$ terms. The spectrum condition then entails:

$$
E\left(\bar{\Delta}+\Gamma_{n}\right)=0
$$

Note, that in the derivation of this result compactness of $\Gamma$ is needed to ensure that the distance between $\Gamma$ and $\bar{V}_{+}$is positive; other shapes of $\Gamma$ are possible as long as convexity and the condition $d\left(\Gamma, \bar{V}_{+}\right)>0$ are preserved, e.g. wedges in $C \bar{V}_{+}$. For arbitrary $\boldsymbol{x}_{1}, \ldots, \boldsymbol{x}_{n} \in \mathbb{R}^{s}$ all the operators $\alpha_{\boldsymbol{x}_{i}}\left(L_{0}\right), i=1, \ldots, n$, belong to $\widetilde{\mathfrak{A}}(\Gamma)$ whilst their product $\prod_{i=1}^{n} \alpha_{x_{i}}\left(L_{0}\right)$ is an element of $\widetilde{\mathfrak{A}}\left(\Gamma_{n}\right)$, hence by (2.7)

$$
\prod_{i=1}^{n} \alpha_{x_{i}}\left(L_{0}\right) E(\Delta)=E\left(\bar{\Delta}+\Gamma_{n}\right) \prod_{i=1}^{n} \alpha_{x_{i}}\left(L_{0}\right) E(\Delta)=0 .
$$

Now, [15, Lemma 2.2] states that for any $B \in \mathfrak{B}(\mathcal{H})$ and any $k \in \mathbb{N}$

$$
\left\|P_{k} \int_{\boldsymbol{K}} d^{s} x \alpha_{x}\left(B^{*} B\right) P_{k}\right\| \leqslant(k-1) \sup _{\Psi}\left(\int_{\boldsymbol{K}-\boldsymbol{K}} d^{s} x\left\|\left[\alpha_{x}(B), B^{*}\right] \Psi\right\|\right),
$$

where $P_{k}$ is the orthogonal projection onto the intersection of the kernels of $k$-fold products $\prod_{i=1}^{k} \alpha_{\boldsymbol{y}_{i}}(B)$ for arbitrary $\boldsymbol{y}_{1}, \ldots, \boldsymbol{y}_{k} \in \mathbb{R}^{s}, \boldsymbol{K} \subseteq \mathbb{R}^{s}$ is compact and the supremum extends over all unit vectors $\Psi \in P_{k-1} \mathcal{H}$. According to (2.8) $E(\Delta) \leqslant P_{n}$ if we take $B \doteq L_{0}$, so that the following estimate, uniform in $\boldsymbol{K}$, is a consequence of (2.9) combined with almost locality of $L_{0}$ (cf. (2.2b)):

$$
\left\|Q_{\Delta, \boldsymbol{K}}^{\left(L_{0}{ }^{*} L_{0}\right)}\right\|=\left\|E(\Delta) \int_{\boldsymbol{K}} d^{s} x \alpha_{x}\left(L_{0}{ }^{*} L_{0}\right) E(\Delta)\right\| \leqslant(n-1) \int_{\mathbb{R}^{s}} d^{s} x\left\|\left[\alpha_{x}\left(L_{0}\right), L_{0}{ }^{*}\right]\right\| .
$$

The positive operators $\left\{Q_{\Delta, \boldsymbol{K}}^{\left(L_{0}{ }^{*} L_{0}\right)}: \boldsymbol{K} \subseteq \mathbb{R}^{s}\right.$ compact $\}$ thus constitute an increasing net which is bounded in $\mathfrak{B}(\mathcal{H})^{+}$. According to [11, Lemma 2.4.19] this net has a least upper bound in $\mathfrak{B}(\mathcal{H})^{+}$, which is its $\sigma$-strong limit $Q_{\Delta}^{\left(L_{0}{ }^{*} L_{0}\right)}$ and satisfies

$$
\left\|Q_{\Delta}^{\left(L_{0}{ }^{*} L_{0}\right)}\right\| \leqslant(n-1) \int_{\mathbb{R}^{s}} d^{s} x\left\|\left[\alpha_{x}\left(L_{0}\right), L_{0}^{*}\right]\right\|
$$

For $N(\Delta, \Gamma) \doteq n-1$ this is the desired estimate (2.6). 
The $\sigma$-weak topology of $\mathfrak{B}(\mathcal{H})$ is induced by the positive normal functionals of the space $\mathfrak{B}(\mathcal{H})_{*}^{+}$, so that integrability of $\boldsymbol{x} \mapsto E(\Delta) \alpha_{\boldsymbol{x}}\left(L_{0}{ }^{*} L_{0}\right) E(\Delta)$ in the $\sigma$-weak topology is implied by integrability of the functions

$$
\boldsymbol{x} \mapsto\left|\psi\left(E(\Delta) \alpha_{x}\left(L_{0}{ }^{*} L_{0}\right) E(\Delta)\right)\right|=\psi\left(E(\Delta) \alpha_{x}\left(L_{0}{ }^{*} L_{0}\right) E(\Delta)\right)
$$

for any $\psi \in \mathfrak{B}(\mathcal{H})_{*}^{+}$. Now, given any compact subset $\boldsymbol{K}$ of $\mathbb{R}^{s}$, there holds the estimate

$$
\begin{aligned}
& \int_{\boldsymbol{K}} d^{s} x\left|\psi\left(E(\Delta) \alpha_{x}\left(L_{0}{ }^{*} L_{0}\right) E(\Delta)\right)\right|=\int_{\boldsymbol{K}} d^{s} x \psi\left(E(\Delta) \alpha_{x}\left(L_{0}{ }^{*} L_{0}\right) E(\Delta)\right) \\
& =\psi\left(\int_{\boldsymbol{K}} d^{s} x E(\Delta) \alpha_{x}\left(L_{0}{ }^{*} L_{0}\right) E(\Delta)\right) \leqslant\|\psi\|\left\|Q_{\Delta, \boldsymbol{K}}^{\left(L_{0}{ }^{*} L_{0}\right)}\right\| \leqslant\|\psi\|\left\|Q_{\Delta}^{\left(L_{0}{ }^{*} L_{0}\right)}\right\|,
\end{aligned}
$$

and, as a consequence of the Monotone Convergence Theorem [26, II.2.7], the functions $\boldsymbol{x} \mapsto\left|\psi\left(E(\Delta) \alpha_{\boldsymbol{x}}\left(L_{0}{ }^{*} L_{0}\right) E(\Delta)\right)\right|$ indeed turn out to be integrable for any $\psi \in$ $\mathfrak{B}(\mathcal{H})_{*}^{+}$. Thus the integral of the mapping $\boldsymbol{x} \mapsto E(\Delta) \alpha_{x}\left(L_{0}{ }^{*} L_{0}\right) E(\Delta)$ with respect to the $\sigma$-weak topology exists (cf. [26, II.6.2]) and, through an application of Lebesgue's Dominated Convergence Theorem [26, II.5.6], is seen to be the $\sigma$-weak limit of the net of operators $Q_{\Delta, \boldsymbol{K}}^{\left(L_{0}{ }^{*} L_{0}\right)}$ which coincides with the $\sigma$-strong limit $Q_{\Delta}^{\left(L_{0}{ }^{*} L_{0}\right)}$ established above. Formally

$$
Q_{\Delta}^{\left(L_{0}{ }^{*} L_{0}\right)}=\sigma \text {-weak } \int_{\mathbb{R}^{s}} d^{s} x E(\Delta) \alpha_{x}\left(L_{0}{ }^{*} L_{0}\right) E(\Delta),
$$

which is the last of the above assertions.

Proposition 2.7. Suppose that $\Delta \subseteq \mathbb{R}^{s+1}$ is a bounded Borel set.

(i) Let $L \in \mathfrak{L}$ be arbitrary, then the net of operators for compact $\boldsymbol{K} \subseteq \mathbb{R}^{s}$

$$
\begin{aligned}
Q_{\Delta, \boldsymbol{K}}^{\left(L^{*} L\right)} & \doteq E(\Delta) \int_{\boldsymbol{K}} d^{s} x \alpha_{x}\left(L^{*} L\right) E(\Delta) \\
& =\int_{\boldsymbol{K}} d^{s} x E(\Delta) \alpha_{x}\left(L^{*} L\right) E(\Delta)
\end{aligned}
$$

converges $\sigma$-strongly to $Q_{\Delta}^{\left(L^{*} L\right)} \in \mathfrak{B}(\mathcal{H})^{+}$in the limit $\boldsymbol{K} \nearrow \mathbb{R}^{s}$. Moreover the mapping $\boldsymbol{x} \mapsto E(\Delta) \alpha_{x}\left(L^{*} L\right) E(\Delta)$ is integrable with respect to the $\sigma$-weak topology on $\mathfrak{B}(\mathcal{H})$ and satisfies

$$
Q_{\Delta}^{\left(L^{*} L\right)}=\sigma \text {-weak } \int_{\mathbb{R}^{s}} d^{s} x E(\Delta) \alpha_{x}\left(L^{*} L\right) E(\Delta) .
$$

(ii) Let $C \in \mathfrak{C}$ be arbitrary, then the net of operators indexed by compact $\boldsymbol{K} \subseteq \mathbb{R}^{s}$

$$
\begin{aligned}
Q_{\Delta, \boldsymbol{K}}^{(C)} & \doteq E(\Delta) \int_{\boldsymbol{K}} d^{s} x \alpha_{x}(C) E(\Delta) \\
& =\int_{\boldsymbol{K}} d^{s} x E(\Delta) \alpha_{x}(C) E(\Delta)
\end{aligned}
$$


is $\sigma$-strongly convergent to $Q_{\Delta}^{(C)} \in \mathfrak{B}(\mathcal{H})$ for $\boldsymbol{K} \nearrow \mathbb{R}^{s}$. In addition to this the mapping $\boldsymbol{x} \mapsto E(\Delta) \alpha_{x}(C) E(\Delta)$ is integrable with respect to the $\sigma$-weak topology on $\mathfrak{B}(\mathcal{H})$ and the integral is given by

$$
Q_{\Delta}^{(C)}=\sigma \text {-weak } \int_{\mathbb{R}^{s}} d^{s} x E(\Delta) \alpha_{x}(C) E(\Delta) .
$$

Furthermore

$$
\sup \left\{\int_{\mathbb{R}^{s}} d^{s} x\left|\phi\left(E(\Delta) \alpha_{x}(C) E(\Delta)\right)\right|: \phi \in \mathfrak{B}(\mathcal{H})_{*, 1}\right\}<\infty .
$$

Remark. Note, that relation (2.12) is a sharpened version of (2.5) which, based on heuristic considerations, was the starting point of the present investigation.

Proof. (i) By partition of unity (cf. [40, Satz 8.1]), applied to elements of $\mathfrak{L}_{0}$ which have arbitrary energy-momentum transfer in $\complement \bar{V}_{+}$, any $L \in \mathfrak{L}$ can be written as a finite sum $L=\sum_{j=1}^{m} A_{j} L_{j}$ where the $A_{j}$ belong to $\mathfrak{A}$ and the operators $L_{j} \in \mathfrak{L}_{0}$ have energymomentum transfer in compact and convex subsets $\Gamma_{j}$ of $\complement \bar{V}_{+}$. Since

$$
L^{*} L \leqslant 2^{m-1}\left(\sup _{1 \leqslant j \leqslant m}\left\|A_{j}\right\|^{2}\right) \sum_{j=1}^{m} L_{j}^{*} L_{j}
$$

we infer

$$
Q_{\Delta, \boldsymbol{K}}^{\left(L^{*} L\right)} \leqslant 2^{m-1}\left(\sup _{1 \leqslant j \leqslant m}\left\|A_{j}\right\|^{2}\right) \sum_{j=1}^{m} Q_{\Delta, \boldsymbol{K}}^{\left(L_{j}^{*} L_{j}\right)}
$$

so that by (2.10) the increasing net $\left\{Q_{\Delta, \boldsymbol{K}}^{\left(L^{*} L\right)}: \boldsymbol{K} \subset \mathbb{R}^{s}\right.$ compact $\}$ turns out to be bounded, having a least upper bound in $\mathfrak{B}(\mathcal{H})^{+}$that is its $\sigma$-strong limit $Q_{\Delta}^{\left(L^{*} L\right)}$. Making again use of the above order relation for $L^{*} L$ one arrives at

$$
\psi\left(E(\Delta) \alpha_{x}\left(L^{*} L\right) E(\Delta)\right) \leqslant 2^{m-1}\left(\sup _{1 \leqslant j \leqslant m}\left\|A_{j}\right\|^{2}\right) \sum_{j=1}^{m} \psi\left(E(\Delta) \alpha_{x}\left(L_{j}{ }^{*} L_{j}\right) E(\Delta)\right)
$$

for any $\psi \in \mathfrak{B}(\mathcal{H})_{*}^{+}$and any $\boldsymbol{x} \in \mathbb{R}^{s}$, where the right-hand side of this relation is integrable as was shown in the proof of Proposition 2.6. Then the reasoning applied there establishes the $\sigma$-weak integrability of $x \mapsto E(\Delta) \alpha_{x}\left(L^{*} L\right) E(\Delta)$ together with the relation

$$
Q_{\Delta}^{\left(L^{*} L\right)}=\sigma \text {-weak } \int_{\mathbb{R}^{s}} d^{s} x E(\Delta) \alpha_{x}\left(L^{*} L\right) E(\Delta)
$$

(ii) Consider $C_{0}=L_{1}{ }^{*} L_{2} \in \mathfrak{C}$ with $L_{1}, L_{2} \in \mathfrak{L}$. By polarization

$$
C_{0}=\frac{1}{4} \sum_{k=0}^{3} i^{-k}\left(L_{1}+i^{k} L_{2}\right)^{*}\left(L_{1}+i^{k} L_{2}\right)=\frac{1}{4} \sum_{k=0}^{3} i^{-k} L^{(k)^{*}} L^{(k)},
$$


where $L^{(k)} \doteq L_{1}+i^{k} L_{2} \in \mathfrak{L}$ for $k=0, \ldots, 3$, and according to (i)

$$
\begin{aligned}
Q_{\Delta, \boldsymbol{K}}^{\left(C_{0}\right)}=E(\Delta) \int_{\boldsymbol{K}} d^{s} x & \alpha_{x}\left(C_{0}\right) E(\Delta) \\
= & \frac{1}{4} \sum_{k=0}^{3} i^{-k}\left(E(\Delta) \int_{\boldsymbol{K}} d^{s} x \alpha_{x}\left(L^{(k)^{*}} L^{(k)}\right) E(\Delta)\right)=\frac{1}{4} \sum_{k=0}^{3} i^{-k} Q_{\Delta, \boldsymbol{K}}^{\left(L^{(k) *} L^{(k)}\right)}
\end{aligned}
$$

converges $\sigma$-strongly to

$$
Q_{\Delta}^{\left(C_{0}\right)} \doteq \frac{1}{4} \sum_{k=0}^{3} i^{-k} Q_{\Delta}^{\left(L^{(k)^{*}} L^{(k)}\right)} .
$$

Now, let $\phi$ be a normal functional on $\mathfrak{B}(\mathcal{H})$. By polar decomposition (cf. [54, Theorem III.4.2(i), Proposition III.4.6]) there exist a partial isometry $V \in \mathfrak{B}(\mathcal{H})$ and a positive normal functional $|\phi|$ subject to the relation $\||\phi|\|=\|\phi\|$, such that $\phi()=.|\phi|(. V)$, allowing for the following estimate $\left(\boldsymbol{x} \in \mathbb{R}^{s}\right.$ arbitrary):

$$
\begin{aligned}
& 2\left|\phi\left(E(\Delta) \alpha_{x}\left(C_{0}\right) E(\Delta)\right)\right|=2|| \phi\left|\left(E(\Delta) \alpha_{x}\left(L_{1}{ }^{*} L_{2}\right) E(\Delta) V\right)\right| \\
& \leqslant 2 \sqrt{|\phi|\left(E(\Delta) \alpha_{x}\left(L_{1}{ }^{*} L_{1}\right) E(\Delta)\right)} \sqrt{|\phi|\left(V^{*} E(\Delta) \alpha_{x}\left(L_{2}{ }^{*} L_{2}\right) E(\Delta) V\right)} \\
& \quad=\inf _{\lambda>0}\left(\lambda^{-1}|\phi|\left(E(\Delta) \alpha_{x}\left(L_{1}{ }^{*} L_{1}\right) E(\Delta)\right)+\lambda|\phi|\left(V^{*} E(\Delta) \alpha_{x}\left(L_{2}{ }^{*} L_{2}\right) E(\Delta) V\right)\right),
\end{aligned}
$$

where we made use of the fact that $2 \sqrt{a b}=\inf _{\lambda>0}\left(\lambda^{-1} a+\lambda b\right)$ for any $a, b \geqslant 0$. Now, from the first part of this Proposition we infer that it is possible to integrate the above expression over all of $\mathbb{R}^{s}$ to get for any $\lambda>0$ the estimate

$$
2 \int_{\mathbb{R}^{s}} d^{s} x\left|\phi\left(E(\Delta) \alpha_{x}\left(C_{0}\right) E(\Delta)\right)\right| \leqslant \lambda^{-1}\|\phi\|\left\|Q_{\Delta}^{\left(L_{1}{ }^{*} L_{1}\right)}\right\|+\lambda\|\phi\|\left\|Q_{\Delta}^{\left(L_{2}{ }^{*} L_{2}\right)}\right\| .
$$

Note, that the normal functionals $\phi$ and the $\sigma$-weak integrals commute due to [26, Proposition II.5.7 adapted to integrals in locally convex spaces]. Taking the infimum with respect to $\lambda$ one finally arrives at

$$
\int_{\mathbb{R}^{s}} d^{s} x\left|\phi\left(E(\Delta) \alpha_{x}\left(C_{0}\right) E(\Delta)\right)\right| \leqslant\|\phi\|\left\|Q_{\Delta}^{\left(L_{1}{ }^{*} L_{1}\right)}\right\|^{1 / 2}\left\|Q_{\Delta}^{\left(L_{2}{ }^{*} L_{2}\right)}\right\|^{1 / 2} .
$$

This relation is valid for any normal functional in $\mathfrak{B}(\mathcal{H})_{*}$, so that the $\sigma$-weak integrability of $\boldsymbol{x} \mapsto E(\Delta) \alpha_{x}\left(C_{0}\right) E(\Delta)$ is established, the relation

$$
Q_{\Delta}^{\left(C_{0}\right)}=\sigma \text {-weak } \int_{\mathbb{R}^{s}} d^{s} x E(\Delta) \alpha_{x}\left(C_{0}\right) E(\Delta)
$$

being an immediate consequence (cf. the proof of Proposition 2.6). Another fact implied by inequality $(2.14)$ is the estimate

$$
\sup \left\{\int_{\mathbb{R}^{s}} d^{s} x\left|\phi\left(E(\Delta) \alpha_{x}\left(C_{0}\right) E(\Delta)\right)\right|: \phi \in \mathfrak{B}(\mathcal{H})_{*, 1}\right\} \leqslant\left\|Q_{\Delta}^{\left(L_{1}{ }^{*} L_{1}\right)}\right\|^{1 / 2}\left\|Q_{\Delta}^{\left(L_{2}{ }^{*} L_{2}\right)}\right\|^{1 / 2} .
$$

Since any $C \in \mathfrak{C}$ is a linear combination of operators of the form $C_{0}$, the above relations (2.13) through (2.16) are easily generalized to establish the second part of the Proposition. 
The preceding result suggests the introduction of topologies on the left ideal $\mathfrak{L}$ and on the ${ }^{*}$-algebra $\mathfrak{C}$, respectively, using specific seminorms indexed by bounded Borel subsets $\Delta$ of $\mathbb{R}^{s+1}$.

Definition 2.8. (a) The left ideal $\mathfrak{L}$ is equipped with a family of seminorms $q_{\Delta}$ via

$$
q_{\Delta}(L) \doteq\left\|Q_{\Delta}^{\left(L^{*} L\right)}\right\|^{1 / 2}, \quad L \in \mathfrak{L} .
$$

(b) The ${ }^{*}$-algebra $\mathfrak{C}$ is furnished with seminorms $p_{\Delta}$ by assigning

$$
p_{\Delta}(C) \doteq \sup \left\{\int_{\mathbb{R}^{s}} d^{s} x\left|\phi\left(E(\Delta) \alpha_{x}(C) E(\Delta)\right)\right|: \phi \in \mathfrak{B}(\mathcal{H})_{*, 1}\right\}, \quad C \in \mathfrak{C} .
$$

(c) The completions of the locally convex (Hausdorff) spaces $\left(\mathfrak{L}, \mathfrak{T}_{q}\right)$ and $\left(\mathfrak{C}, \mathfrak{T}_{p}\right)$ arising from topologization by these seminorms are denoted $\left(\overline{\mathfrak{L}}, \overline{\mathfrak{T}}_{q}\right)$ and $\left(\overline{\mathfrak{C}}, \overline{\mathfrak{T}}_{p}\right)$, respectively. Accordingly, the complete locally convex subspace of $\overline{\mathfrak{L}}$ generated by $\mathfrak{L}_{0}$ is designated as $\left(\overline{\mathfrak{L}}_{0}, \overline{\mathfrak{T}}_{q}\right)$.

(d) The completions of the locally convex spaces $\left(\mathfrak{L}, \mathfrak{T}_{q}^{u}\right)$ and $\left(\mathfrak{C}, \mathfrak{T}_{p}^{u}\right)$ arising from topologization by all the seminorms $q_{\Delta}$ and $p_{\Delta}$, respectively, together with the initial uniform (norm) topology inherited from the quasi-local algebra $\mathfrak{A}$ are denoted $\left(\mathfrak{A}_{\mathfrak{L}}, \overline{\mathfrak{T}}_{q}^{u}\right)$ and $\left(\mathfrak{A}_{\mathfrak{C}}, \overline{\mathfrak{T}}_{p}^{u}\right)$.

Remark. (i) Let $\mathfrak{B}(\mathcal{H})_{*}^{+}$denote the positive cone in $\mathfrak{B}(\mathcal{H})_{*}$, then for any $L \in \mathfrak{L}$

$$
q_{\Delta}(L)^{2}=\sup \left\{\int_{\mathbb{R}^{s}} d^{s} x \omega\left(E(\Delta) \alpha_{x}\left(L^{*} L\right) E(\Delta)\right): \omega \in \mathfrak{B}(\mathcal{H})_{*, 1}^{+}\right\}
$$

a formulation that will frequently be used.

(ii) The seminorm properties of $q_{\Delta}$ and $p_{\Delta}$ are easily checked. To establish the subadditivity of $q_{\Delta}$ one has to observe that

$$
\begin{aligned}
q_{\Delta}\left(L_{1}+L_{2}\right)^{2} \leqslant q_{\Delta}\left(L_{1}\right)^{2} & +\inf _{\lambda>0}\left[\lambda^{-1} q_{\Delta}\left(L_{1}\right)^{2}+\lambda q_{\Delta}\left(L_{2}\right)^{2}\right]+q_{\Delta}\left(L_{2}\right)^{2} \\
& =q_{\Delta}\left(L_{1}\right)^{2}+2 q_{\Delta}\left(L_{1}\right) q_{\Delta}\left(L_{2}\right)+q_{\Delta}\left(L_{2}\right)^{2}=\left(q_{\Delta}\left(L_{1}\right)+q_{\Delta}\left(L_{2}\right)\right)^{2},
\end{aligned}
$$

where we made use of the fact that $L_{1}{ }^{*} L_{2}+L_{2}{ }^{*} L_{1} \leqslant \lambda^{-1} L_{1}{ }^{*} L_{1}+\lambda L_{2}{ }^{*} L_{2}$ for any $\lambda>0$ and $L_{1}, L_{2} \in \mathfrak{L}$.

(iii) The Hausdorff property of the locally convex spaces $\left(\mathfrak{L}, \mathfrak{T}_{q}\right)$ and $\left(\mathfrak{C}, \mathfrak{T}_{p}\right)$ can be established using the fact that vectors corresponding to states of bounded energy constitute a dense subspace of $\mathcal{H}$. From the very definition of the seminorms $q_{\Delta}$ and $p_{\Delta}$ we infer that the conditions $q_{\Delta}(L)=0$ and $p_{\Delta}(C)=0, L \in \mathfrak{L}, C \in \mathfrak{C}$, imply $L E(\Delta)=0$ and $E(\Delta) C E(\Delta)=0$ for any bounded Borel set $\Delta$, since the integrands occurring in (2.17c) and (2.17b) vanish identically on $\mathbb{R}^{s}$, and $\mathfrak{B}(\mathcal{H})_{*, 1}^{+}$as well as $\mathfrak{B}(\mathcal{H})_{*, 1}$ are separating sets of functionals for $\mathfrak{B}(\mathcal{H})$. By the density property just mentioned, it then follows that $L=0$ and $C=0$, and the nets of seminorms turn out to separate the elements of the left ideal $\mathfrak{L}$ and the *-algebra $\mathfrak{C}$, respectively.

(iv) The completions $\left(\overline{\mathfrak{L}}, \overline{\mathfrak{T}}_{q}\right)$ and $\left(\overline{\mathfrak{C}}, \overline{\mathfrak{T}}_{p}\right)$ as well as $\left(\mathfrak{A}_{\mathfrak{L}}, \overline{\mathfrak{T}}_{q}^{u}\right)$ and $\left(\mathfrak{A}_{\mathfrak{C}}, \overline{\mathfrak{T}}_{p}^{u}\right)$ are again locally convex spaces with topologies defined by the unique extensions of the seminorms 
$q_{\Delta}$ and $p_{\Delta}$ and of the norm $\|$.$\| to \overline{\mathfrak{L}}, \mathfrak{A}_{\mathfrak{L}}$ and $\overline{\mathfrak{C}}, \mathfrak{A}_{\mathfrak{C}}$, respectively [44, Chapter Four, $\S 18,4$.]. Therefore, in the sequel, we shall apply these seminorms to elements of the completions without special mention. Depending on the relation between the underlying uniform structures as being finer or coarser, we infer that $\mathfrak{A}_{\mathfrak{L}} \subseteq \overline{\mathfrak{L}}$ and $\mathfrak{A}_{\mathfrak{C}} \subseteq \overline{\mathfrak{C}}$. Furthermore $\mathfrak{A}_{\mathfrak{L}}$ and $\mathfrak{A}_{\mathfrak{C}}$ are uniformly closed subspaces of the quasi-local algebra $\mathfrak{A}$.

\subsection{Characteristics of the Spectral Seminorms}

The investigations of the subsequent chapters very much depend on special properties of the seminorms defined above, so these are collected in this section. Interesting in their own right as they may be, we are, in the present context, not aiming at utmost generality of statements, but have future applications in mind.

\subsubsection{Basic Properties}

Proposition 2.9. The families of seminorms $q_{\Delta}$ and $p_{\Delta}$ on $\overline{\mathfrak{L}}$ and $\overline{\mathfrak{C}}$, respectively, where the symbols $\Delta$ denote bounded Borel sets, constitute nets with respect to the inclusion relation. For any $\Delta$ and $\Delta^{\prime}$ we have

$$
\begin{array}{lll}
\Delta \subseteq \Delta^{\prime} & \Rightarrow & q_{\Delta}(L) \leqslant q_{\Delta^{\prime}}(L), \quad L \in \overline{\mathfrak{L}}, \\
\Delta \subseteq \Delta^{\prime} & \Rightarrow & p_{\Delta}(C) \leqslant p_{\Delta^{\prime}}(C), \quad C \in \overline{\mathfrak{C}} .
\end{array}
$$

Proof. For the $q_{\Delta}$-seminorms on $\overline{\mathfrak{L}}$ the assertion follows from the order relation for operators in $\mathfrak{B}(\mathcal{H})^{+}$. Let $L$ belong to the left ideal $\mathfrak{L}$, then

$$
Q_{\Delta}^{\left(L^{*} L\right)} \leqslant Q_{\Delta^{\prime}}^{\left(L^{*} L\right)}
$$

which by Definition 2.8 has the consequence

$$
q_{\Delta}(L)^{2}=\left\|Q_{\Delta}^{\left(L^{*} L\right)}\right\| \leqslant\left\|Q_{\Delta^{\prime}}^{\left(L^{*} L\right)}\right\|=q_{\Delta^{\prime}}(L)^{2} .
$$

This relation extends by continuity of the seminorms to all of $\overline{\mathfrak{L}}$.

In case of the $p_{\Delta}$-topologies, note that for any Borel set $\Delta$ the functional $\phi^{E(\Delta)}$, defined through $\phi^{E(\Delta)}(.) \doteq \phi(E(\Delta)$. $E(\Delta))$, belongs to $\mathfrak{B}(\mathcal{H})_{*, 1}$ if $\phi$ does. From this we infer, since moreover $\Delta \subseteq \Delta^{\prime}$ implies $E(\Delta)=E(\Delta) E\left(\Delta^{\prime}\right)=E\left(\Delta^{\prime}\right) E(\Delta)$, that

$$
\begin{aligned}
\left\{\int_{\mathbb{R}^{s}} d^{s} x\left|\phi\left(E(\Delta) \alpha_{x}(C) E(\Delta)\right)\right|: \phi\right. & \left.\in \mathfrak{B}(\mathcal{H})_{*, 1}\right\} \\
& \subseteq\left\{\int_{\mathbb{R}^{s}} d^{s} x\left|\phi\left(E\left(\Delta^{\prime}\right) \alpha_{x}(C) E\left(\Delta^{\prime}\right)\right)\right|: \phi \in \mathfrak{B}(\mathcal{H})_{*, 1}\right\}
\end{aligned}
$$

for any $C \in \mathfrak{C}$ and thus, by $(2.17 \mathrm{~b})$, that $p_{\Delta}(C) \leqslant p_{\Delta^{\prime}}(C)$, a relation which by continuity of the seminorms is likewise valid for any operator in the completion $\overline{\mathfrak{C}}$.

The continuous extensions of the seminorms $q_{\Delta}$ and $p_{\Delta}$ to $\overline{\mathfrak{L}}$ and $\overline{\mathfrak{C}}$, respectively, can be explicitly computed on the subspaces $\mathfrak{A}_{\mathfrak{L}}$ and $\mathfrak{A}_{\mathfrak{C}}$ of $\mathfrak{A}$. 
Lemma 2.10. Let $\Delta$ denote an arbitrary bounded Borel subset of $\mathbb{R}^{s+1}$.

(i) For any $L \in \mathfrak{A}_{\mathfrak{L}}$ we have

$$
q_{\Delta}(L)=\sup \left\{\int_{\mathbb{R}^{s}} d^{s} x \omega\left(E(\Delta) \alpha_{x}\left(L^{*} L\right) E(\Delta)\right): \omega \in \mathfrak{B}(\mathcal{H})_{*, 1}^{+}\right\}^{1 / 2}
$$

(ii) For any $C \in \mathfrak{A}_{\mathfrak{C}}$ there holds the relation

$$
p_{\Delta}(C)=\sup \left\{\int_{\mathbb{R}^{s}} d^{s} x\left|\phi\left(E(\Delta) \alpha_{x}(C) E(\Delta)\right)\right|: \phi \in \mathfrak{B}(\mathcal{H})_{*, 1}\right\}
$$

Proof. (i) Note, that we can define a linear subspace $\mathfrak{A}_{q^{\prime}}$ of $\mathfrak{A}$ consisting of all those operators $L^{\prime}$ which fulfill

$$
q_{\Delta}^{\prime}\left(L^{\prime}\right)^{2} \doteq \sup \left\{\int_{\mathbb{R}^{s}} d^{s} x \omega\left(E(\Delta) \alpha_{x}\left(L^{*} L^{\prime}\right) E(\Delta)\right): \omega \in \mathfrak{B}(\mathcal{H})_{*, 1}^{+}\right\}<\infty
$$

for any bounded Borel set $\Delta$. On this space the mappings $q_{\Delta}^{\prime}$ act as seminorms whose restrictions to $\mathfrak{L}$ coincide with $q_{\Delta}$ (cf. the Remark following Definition 2.8). Now let $L \in \mathfrak{A}_{\mathfrak{L}}$ be arbitrary. Given a bounded Borel set $\Delta$ we can then find a sequence $\left\{L_{n}\right\}_{n \in \mathbb{N}}$ in $\mathfrak{L}$ satisfying

$$
\lim _{n \rightarrow \infty} q_{\Delta}\left(L-L_{n}\right)=0 \quad \text { and } \quad \lim _{n \rightarrow \infty}\left\|L-L_{n}\right\|=0 .
$$

The second equation implies

$$
\lim _{n \rightarrow \infty}\left\|L E(\Delta)-L_{n} E(\Delta)\right\|=0
$$

so that Lebesgue's Dominated Convergence Theorem can be applied to get for any functional $\omega \in \mathfrak{B}(\mathcal{H})_{*, 1}^{+}$and any compact $\boldsymbol{K} \subseteq \mathbb{R}^{s}$

$$
\int_{K} d^{s} x \omega\left(E(\Delta) \alpha_{x}\left(L^{*} L\right) E(\Delta)\right)=\lim _{n \rightarrow \infty} \int_{K} d^{s} x \omega\left(E(\Delta) \alpha_{x}\left(L_{n}{ }^{*} L_{n}\right) E(\Delta)\right) .
$$

According to $(2.17 \mathrm{c}$ ) each term in the sequence on the right-hand side is majorized by the corresponding $q_{\Delta}\left(L_{n}\right)^{2}$ and this sequence in turn converges to $q_{\Delta}(L)^{2}$ by assumption, so that in passing from $\boldsymbol{K}$ to $\mathbb{R}^{s}$ and to the supremum over all $\omega \in \mathfrak{B}(\mathcal{H})_{*, 1}^{+}$we get

$$
\sup \left\{\int_{\mathbb{R}^{s}} d^{s} x \omega\left(E(\Delta) \alpha_{x}\left(L^{*} L\right) E(\Delta)\right): \omega \in \mathfrak{B}(\mathcal{H})_{*, 1}^{+}\right\} \leqslant q_{\Delta}(L)^{2}
$$

This final estimate shows, by arbitrariness of $L \in \mathfrak{A}_{\mathfrak{L}}$ and the selected $\Delta$, that $\mathfrak{A}_{\mathfrak{L}}$ is a subspace of $\mathfrak{A}_{q^{\prime}}$ and, from $q_{\Delta}^{\prime}\left\lceil\mathfrak{L}=q_{\Delta}\right.$, it eventually follows that for all these $L$ and $\Delta$

$$
q_{\Delta}(L)=\sup \left\{\int_{\mathbb{R}^{s}} d^{s} x \omega\left(E(\Delta) \alpha_{x}\left(L^{*} L\right) E(\Delta)\right): \omega \in \mathfrak{B}(\mathcal{H})_{*, 1}^{+}\right\}^{1 / 2}
$$


(ii) The proof of the second part follows the same lines of thought. We introduce the subspace $\mathfrak{A}_{p^{\prime}} \subseteq \mathfrak{A}$ consisting of operators $C^{\prime}$ satisfying

$$
p_{\Delta}^{\prime}\left(C^{\prime}\right) \doteq \sup \left\{\int_{\mathbb{R}^{s}} d^{s} x\left|\phi\left(E(\Delta) \alpha_{x}\left(C^{\prime}\right) E(\Delta)\right)\right|: \phi \in \mathfrak{B}(\mathcal{H})_{*, 1}\right\}<\infty
$$

for any bounded Borel set $\Delta$ and furnish it with the locally convex topology defined by the seminorms $p_{\Delta}^{\prime}$. An arbitrary $C \in \mathfrak{A}_{\mathfrak{C}}$ is, for given $\Delta$, approximated by a sequence $\left\{C_{n}\right\}_{n \in \mathbb{N}}$ with respect to the norm and the $p_{\Delta}$-topology. As above one has

$$
\lim _{n \rightarrow \infty}\left\|E(\Delta) C E(\Delta)-E(\Delta) C_{n} E(\Delta)\right\|=0
$$

and infers

$$
\sup \left\{\int_{\mathbb{R}^{s}} d^{s} x\left|\phi\left(E(\Delta) \alpha_{x}\left(C^{\prime}\right) E(\Delta)\right)\right|: \phi \in \mathfrak{B}(\mathcal{H})_{*, 1}\right\} \leqslant p_{\Delta}(C) .
$$

This establishes, by arbitraryness of $C \in \mathfrak{A}_{\mathfrak{C}}$ and $\Delta$, that $\mathfrak{A}_{\mathfrak{C}} \subseteq \mathfrak{A}_{p^{\prime}}$, and the equation $p_{\Delta}^{\prime}\left\lceil\mathfrak{C}=p_{\Delta}\right.$ implies that for these $C$ and $\Delta$

$$
p_{\Delta}(C)=\sup \left\{\int_{\mathbb{R}^{s}} d^{s} x\left|\phi\left(E(\Delta) \alpha_{x}(C) E(\Delta)\right)\right|: \phi \in \mathfrak{B}(\mathcal{H})_{*, 1}\right\} .
$$

An immediate consequence of this result is the subsequent lemma, which in some way reverts the arguments given in the concluding remark of the last section in order to establish the Hausdorff property for $\left(\mathfrak{L}, \mathfrak{T}_{q}\right)$ and $\left(\mathfrak{C}, \mathfrak{T}_{p}\right)$.

Lemma 2.11. Let $\Delta$ be a bounded Borel set.

(i) For $L \in \mathfrak{A}_{\mathfrak{L}}$ with $L E(\Delta)=0$ there holds $q_{\Delta}(L)=0$.

(ii) If $C \in \mathfrak{A}_{\mathfrak{C}}$ satisfies $E(\Delta) C E(\Delta)=0$ one has $p_{\Delta}(C)=0$.

Next we deal with an implication of the fact, that $\mathfrak{L}$ is an ideal of the $C^{*}$-algebra $\mathfrak{A}$, and clarify the relationship between the seminorms $q_{\Delta}$ and $p_{\Delta}$.

Lemma 2.12. Let $\Delta$ denote bounded Borel subsets of $\mathbb{R}^{s+1}$.

(i) $\mathfrak{A}_{\mathfrak{L}}$ is a left ideal of the quasi-local algebra $\mathfrak{A}$ and satisfies

$$
q_{\Delta}(A L) \leqslant\|A\| q_{\Delta}(L)
$$

for any $L \in \mathfrak{A}_{\mathfrak{L}}$ and $A \in \mathfrak{A}$.

(ii) Let $L_{i}, i=1,2$, be operators in $\mathfrak{A}_{\mathfrak{L}}$ and $A \in \mathfrak{A}$, then $L_{1}{ }^{*} A L_{2}$ belongs to $\mathfrak{A}_{\mathfrak{C} .}$ If in addition the operators $L_{i}$ have energy-momentum transfer in $\Gamma_{i} \subseteq \mathbb{R}^{s+1}$ and $\Delta_{i}$ are Borel subsets of $\mathbb{R}^{s+1}$ containing $\Delta+\Gamma_{i}$, respectively, then

$$
p_{\Delta}\left(L_{1}{ }^{*} A L_{2}\right) \leqslant\left\|E\left(\Delta_{1}\right) A E\left(\Delta_{2}\right)\right\| q_{\Delta}\left(L_{1}\right) q_{\Delta}\left(L_{2}\right) .
$$

Proof. (i) For any $L \in \mathfrak{A}_{\mathfrak{L}} \subseteq \mathfrak{A}$ and arbitrary $A \in \mathfrak{A}$ the relation $L^{*} A^{*} A L \leqslant\|A\|^{2} L^{*} L$ leads to the estimate

$$
\int_{\mathbb{R}^{s}} d^{s} x \omega\left(E(\Delta) \alpha_{x}\left(L^{*} A^{*} A L\right) E(\Delta)\right) \leqslant\|A\|^{2} \int_{\mathbb{R}^{s}} d^{s} x \omega\left(E(\Delta) \alpha_{x}\left(L^{*} L\right) E(\Delta)\right)
$$


for any $\omega \in \mathfrak{B}(\mathcal{H})_{*, 1}^{+}$and thus, by (2.18a) and the notation of the proof of Lemma 2.10, to

$$
\begin{aligned}
q_{\Delta}^{\prime}(A L)= & \sup \left\{\int_{\mathbb{R}^{s}} d^{s} x \omega\left(E(\Delta) \alpha_{x}\left(L^{*} A^{*} A L\right) E(\Delta)\right): \omega \in \mathfrak{B}(\mathcal{H})_{*, 1}^{+}\right\}^{1 / 2} \\
& \leqslant\|A\| \sup \left\{\int_{\mathbb{R}^{s}} d^{s} x \omega\left(E(\Delta) \alpha_{x}\left(L^{*} L\right) E(\Delta)\right): \omega \in \mathfrak{B}(\mathcal{H})_{*, 1}^{+}\right\}^{1 / 2}=\|A\| q_{\Delta}(L) .
\end{aligned}
$$

This shows that $A L$ belongs to $\mathfrak{A}_{\mathfrak{L}}$ and at the same time that the seminorm $q_{\Delta}^{\prime}$ (on $\mathfrak{A}_{q^{\prime}}$ ) can be replaced by $q_{\Delta}$ to yield (2.19).

(ii) Let $\phi$ be a normal functional on $\mathfrak{B}(\mathcal{H})$ with $\|\phi\| \leqslant 1$. By polar decomposition there exist a partial isometry $V$ and a positive normal functional $|\phi|$ with $\||\phi|\| \leqslant 1$ such that $\phi()=.|\phi|(. V)$. Then

$$
\begin{aligned}
& \left|\phi\left(E(\Delta) \alpha_{x}\left(L_{1}{ }^{*} A L_{2}\right) E(\Delta)\right)\right| \\
& =|\phi|\left(E(\Delta) \alpha_{x}\left(L_{1}{ }^{*}\right) E\left(\Delta_{1}\right) \alpha_{x}(A) E\left(\Delta_{2}\right) \alpha_{x}\left(L_{2}\right) E(\Delta) V\right) \\
& \leqslant\left\|E\left(\Delta_{1}\right) \alpha_{x}(A) E\left(\Delta_{2}\right)\right\| \sqrt{|\phi|\left(E(\Delta) \alpha_{x}\left(L_{1}{ }^{*} L_{1}\right) E(\Delta)\right)} \sqrt{|\phi|\left(V^{*} E(\Delta) \alpha_{x}\left(L_{2}{ }^{*} L_{2}\right) E(\Delta) V\right)}
\end{aligned}
$$

for any $\boldsymbol{x} \in \mathbb{R}^{s}$ and the method used in the proof of Proposition 2.7 can be applied to get, in analogy to (2.16),

$$
\begin{aligned}
\sup \left\{\int_{\mathbb{R}^{s}} d^{s} x\left|\phi\left(E(\Delta) \alpha_{x}\left(L_{1}{ }^{*} A L_{2}\right) E(\Delta)\right)\right|: \phi \in \mathfrak{B}(\mathcal{H})_{*, 1}\right\} & \\
& \leqslant\left\|E\left(\Delta_{1}\right) A E\left(\Delta_{2}\right)\right\| q_{\Delta}\left(L_{1}\right) q_{\Delta}\left(L_{2}\right),
\end{aligned}
$$

where we made use of (2.18a). According to the notation introduced in the proof of Lemma 2.10 this result expressed in terms of the seminorm $p_{\Delta}^{\prime}$ on $\mathfrak{A}_{p^{\prime}}$ reads

$$
p_{\Delta}^{\prime}\left(L_{1}^{*} A L_{2}\right) \leqslant\left\|E\left(\Delta_{1}\right) A E\left(\Delta_{2}\right)\right\| q_{\Delta}\left(L_{1}\right) q_{\Delta}\left(L_{2}\right),
$$

from which we infer, as in the first part of the present proof, not only that $L_{1}{ }^{*} A L_{2}$ is an element of $\mathfrak{A}_{\mathfrak{C}}$ but also that $p_{\Delta}^{\prime}$ can be substituted by $p_{\Delta}$ to give (2.20).

The second part of the above lemma means that the product $L_{1}{ }^{*} L_{2}$, defined by two operators $L_{1}, L_{2} \in \mathfrak{A}_{\mathfrak{L}}$, is continuous with respect to the locally convex spaces (cf. [44, Chapter Four, § 18,3.(5)]) $\left(\mathfrak{A}_{\mathfrak{L}}, \overline{\mathfrak{T}}_{q}^{u}\right) \times\left(\mathfrak{A}_{\mathfrak{L}}, \overline{\mathfrak{T}}_{q}^{u}\right)$ and $\left(\mathfrak{A}_{\mathfrak{C}}, \overline{\mathfrak{T}}_{p}^{u}\right)$.

Corollary 2.13. The sesquilinear mapping on the topological product of the locally convex space $\left(\mathfrak{A}_{\mathfrak{L}}, \overline{\mathfrak{T}}_{q}^{u}\right)$ with itself, defined by

$$
\mathfrak{A}_{\mathfrak{L}} \times \mathfrak{A}_{\mathfrak{L}} \ni\left(L_{1}, L_{2}\right) \mapsto L_{1}{ }^{*} L_{2} \in \mathfrak{A}_{\mathfrak{C}}
$$

is continuous with respect to the respective locally convex topologies.

In the special case of coincidence of both operators $\left(L_{1}=L_{2}=L\right)$ it turns out that $p_{\Delta}\left(L^{*} L\right)$ equals the square of $q_{\Delta}(L)$. Another result involving the operation of adjunction is the fact, that this mapping leaves the $p_{\Delta}$-seminorms invariant.

Lemma 2.14. Let $\Delta$ denote the bounded Borel sets in $\mathbb{R}^{s+1}$. 
(i) For any operator $L \in \mathfrak{A}_{\mathfrak{L}}$ there hold the relations

$$
p_{\Delta}\left(L^{*} L\right)=q_{\Delta}(L)^{2} .
$$

(ii) Let $C$ be an element of $\mathfrak{A}_{\mathfrak{C},}$, then $C^{*}$ lies in $\mathfrak{A}_{\mathfrak{C}}$, too, and satisfies

$$
p_{\Delta}\left(C^{*}\right)=p_{\Delta}(C) .
$$

Proof. (i) According to Lemma 2.10, we have for any $L \in \mathfrak{A}_{\mathfrak{L}}$

$$
\begin{aligned}
& q_{\Delta}(L)^{2}=\sup \left\{\int_{\mathbb{R}^{s}} d^{s} x \omega\left(E(\Delta) \alpha_{x}\left(L^{*} L\right) E(\Delta)\right): \omega \in \mathfrak{B}(\mathcal{H})_{*, 1}^{+}\right\} \\
& \quad \leqslant \sup \left\{\int_{\mathbb{R}^{s}} d^{s} x\left|\phi\left(E(\Delta) \alpha_{x}\left(L^{*} L\right) E(\Delta)\right)\right|: \phi \in \mathfrak{B}(\mathcal{H})_{*, 1}\right\}=p_{\Delta}\left(L^{*} L\right),
\end{aligned}
$$

whereas the reverse inequality is a consequence of Lemma 2.12. This proves the assertion.

(ii) Note, that $\mathfrak{B}(\mathcal{H})_{*, 1}$ is invariant under the operation of taking adjoints defined by $\psi \mapsto \psi^{*}$ with $\psi^{*}(A) \doteq \overline{\psi\left(A^{*}\right)}, A \in \mathfrak{B}(\mathcal{H})$, for any linear functional $\psi$ on $\mathfrak{B}(\mathcal{H})$. Thus

$$
\begin{aligned}
p_{\Delta}^{\prime}\left(C^{*}\right)=\sup \left\{\int_{\mathbb{R}^{s}} d^{s} x\right. & \left.\left|\phi\left(E(\Delta) \alpha_{x}\left(C^{*}\right) E(\Delta)\right)\right|: \phi \in \mathfrak{B}(\mathcal{H})_{*, 1}\right\} \\
= & \sup \left\{\int_{\mathbb{R}^{s}} d^{s} x\left|\phi^{*}\left(E(\Delta) \alpha_{x}(C) E(\Delta)\right)\right|: \phi \in \mathfrak{B}(\mathcal{H})_{*, 1}\right\}=p_{\Delta}^{\prime}(C)
\end{aligned}
$$

for any $C \in \mathfrak{A}_{p^{\prime}}$ (cf. the proof of Lemma 2.10), which is sufficient to establish both of the assertions.

The last statement of this subsection on basic properties of the spectral seminorms establishes their invariance under translations in the $s+1$-dimensional configuration space.

Lemma 2.15. The subspaces $\mathfrak{A}_{\mathfrak{L}}$ and $\mathfrak{A}_{\mathfrak{C}}$ of the quasi-local algebra $\mathfrak{A}$ are invariant under translations. In particular, let $\Delta$ be a bounded Borel set in $\mathbb{R}^{s+1}$ and let $x \in \mathbb{R}^{s+1}$ be arbitrary, then

$$
\begin{aligned}
& q_{\Delta}\left(\alpha_{x}(L)\right)=q_{\Delta}(L), \quad L \in \mathfrak{A}_{\mathfrak{L}} \\
& p_{\Delta}\left(\alpha_{x}(C)\right)=p_{\Delta}(C), \quad C \in \mathfrak{A}_{\mathfrak{C}} .
\end{aligned}
$$

Proof. $\mathfrak{B}(\mathcal{H})_{*, 1}$ as well as its intersection $\mathfrak{B}(\mathcal{H})_{*, 1}^{+}$with the positive cone $\mathfrak{B}(\mathcal{H})_{*}^{+}$are invariant under the mapping $\psi \mapsto \psi^{U}$ defined by $\psi^{U}(.) \doteq \psi\left(U . U^{*}\right)$ for any unitary operator $U \in \mathfrak{B}(\mathcal{H})$ and any linear functional $\psi$ on $\mathfrak{B}(\mathcal{H})$.

(i) Now, $\alpha_{x}\left(L^{*} L\right)=U_{t} \alpha_{x}\left(L^{*} L\right) U_{t}{ }^{*}$ for any $x=(t, \boldsymbol{x}) \in \mathbb{R}^{s+1}$. This implies

$$
\omega\left(E(\Delta) \alpha_{y}\left(\alpha_{x}\left(L^{*} L\right)\right) E(\Delta)\right)=\omega\left(U_{t} E(\Delta) \alpha_{x+y}\left(L^{*} L\right) E(\Delta) U_{t}^{*}\right)
$$

for any $\boldsymbol{y} \in \mathbb{R}^{s}$ and any $\boldsymbol{\omega} \in \mathfrak{B}(\mathcal{H})_{*}$, henceforth

$$
\begin{aligned}
\int_{\mathbb{R}^{s}} d^{s} y \omega\left(E(\Delta) \alpha_{y}\left(\alpha_{x}\left(L^{*} L\right)\right) E(\Delta)\right) \\
\quad=\int_{\mathbb{R}^{s}} d^{s} y \omega\left(U_{t} E(\Delta) \alpha_{x+y}\left(L^{*} L\right) E(\Delta) U_{t}^{*}\right)=\int_{\mathbb{R}^{s}} d^{s} y \omega\left(U_{t} E(\Delta) \alpha_{y}\left(L^{*} L\right) E(\Delta) U_{t}^{*}\right) .
\end{aligned}
$$


Therefore the introductory remark in combination with (2.18a) yields for any $L \in \mathfrak{A}_{\mathfrak{L}}$ :

$$
\begin{aligned}
q_{\Delta}^{\prime}\left(\alpha_{x}(L)\right)^{2}=\sup \left\{\int_{\mathbb{R}^{s}} d^{s} y \omega\left(E(\Delta) \alpha_{y}\left(\alpha_{x}\left(L^{*} L\right)\right) E(\Delta)\right): \omega \in \mathfrak{B}(\mathcal{H})_{*, 1}^{+}\right\} \\
=\sup \left\{\int_{\mathbb{R}^{s}} d^{s} y \omega\left(U_{t} E(\Delta) \alpha_{y}\left(L^{*} L\right) E(\Delta) U_{t}^{*}\right): \omega \in \mathfrak{B}(\mathcal{H})_{*, 1}^{+}\right\} \\
\quad=\sup \left\{\int_{\mathbb{R}^{s}} d^{s} y \omega\left(E(\Delta) \alpha_{y}\left(L^{*} L\right) E(\Delta)\right): \omega \in \mathfrak{B}(\mathcal{H})_{*, 1}^{+}\right\}=q_{\Delta}^{\prime}(L)^{2},
\end{aligned}
$$

which, as in the proof of Lemma 2.12, establishes the assertions.

(ii) The same argument applies to the seminorm $p_{\Delta}^{\prime}$, so that for $C \in \mathfrak{A}_{\mathfrak{C}}$

$$
\begin{aligned}
p_{\Delta}\left(\alpha_{x}(C)\right)= & \sup \left\{\int_{\mathbb{R}^{s}} d^{s} y\left|\phi\left(E(\Delta) \alpha_{y}\left(\alpha_{x}(C)\right) E(\Delta)\right)\right|: \phi \in \mathfrak{B}(\mathcal{H})_{*, 1}\right\} \\
= & \sup \left\{\int_{\mathbb{R}^{s}} d^{s} y\left|\phi\left(U_{t} E(\Delta) \alpha_{y}(C) E(\Delta) U_{t}^{*}\right)\right|: \phi \in \mathfrak{B}(\mathcal{H})_{*, 1}\right\} \\
= & \sup \left\{\int_{\mathbb{R}^{s}} d^{s} y\left|\phi\left(E(\Delta) \alpha_{y}(C) E(\Delta)\right)\right|: \phi \in \mathfrak{B}(\mathcal{H})_{*, 1}\right\}=p_{\Delta}(C) .
\end{aligned}
$$

\subsubsection{Continuity and Differentiability}

The assumed strong continuity of the automorphism group $\left\{\alpha_{(\Lambda, x)}:(\Lambda, x) \in \mathrm{P}_{+}^{\uparrow}\right\}$ acting on the $C^{*}$-algebra $\mathfrak{A}$ carries over to the locally convex spaces $\left(\mathfrak{L}, \mathfrak{T}_{q}\right)$ and $\left(\mathfrak{C}, \mathfrak{T}_{p}\right)$; and even the infinite differentiability of $(\Lambda, x) \mapsto \alpha_{(\Lambda, x)}\left(L_{0}\right)$ for $L_{0} \in \mathfrak{L}_{0}$ is conserved in passing from the uniform topology on $\mathfrak{L}_{0}$ to that induced by the seminorms $q_{\Delta}$.

Proposition 2.16. (i) For fixed $L \in \mathfrak{L}$ the mapping

$$
\Xi_{L}: P_{+}^{\uparrow} \rightarrow \mathfrak{L} \quad(\Lambda, x) \mapsto \Xi_{L}(\Lambda, x) \doteq \alpha_{(\Lambda, x)}(L)
$$

is continuous with respect to the locally convex space $\left(\mathfrak{L}, \mathfrak{T}_{q}\right)$.

(ii) For given $C \in \mathfrak{C}$ the mapping

$$
\Xi_{C}: \mathrm{P}_{+}^{\uparrow} \rightarrow \mathfrak{C} \quad(\Lambda, x) \mapsto \Xi_{C}(\Lambda, x) \doteq \alpha_{(\Lambda, x)}(C)
$$

is continuous with respect to the locally convex space $\left(\mathfrak{C}, \mathfrak{T}_{p}\right)$.

(iii) Let $\mathrm{id}_{\mathfrak{L}_{0}}$ denote the identity mapping

$$
\operatorname{id}_{\mathfrak{L}_{0}}:\left(\mathfrak{L}_{0},\|\cdot\|\right) \rightarrow\left(\mathfrak{L}_{0}, \mathfrak{T}_{q}\right) \quad L_{0} \mapsto \operatorname{id}_{\mathfrak{L}_{0}}\left(L_{0}\right) \doteq L_{0}
$$

on the space $\mathfrak{L}_{0}$, once endowed with the norm topology and once with $\mathfrak{T}_{q}$. Consider furthermore the family $X_{\mathfrak{L}_{0}}$ of infinitely often differentiable mappings:

$$
\begin{gathered}
x_{\mathfrak{L}_{0}} \doteq\left\{\Xi_{L_{0}}: L_{0} \in \mathfrak{L}_{0}\right\}, \\
\Xi_{L_{0}}: \mathrm{P}_{+}^{\uparrow} \rightarrow \mathfrak{L}_{0} \quad(\Lambda, x) \mapsto \Xi_{L_{0}}(\Lambda, x) \doteq \alpha_{(\Lambda, x)}\left(L_{0}\right) .
\end{gathered}
$$

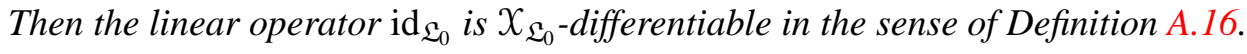


Remark. The last assertion means, due to the invariance of $X_{\mathfrak{L}_{0}}$ under differentiation of arbitrary order, that all the mappings $\Xi_{L_{0}}, L_{0} \in \mathfrak{L}_{0}$, are infinitely often differentiable in the locally convex space $\left(\mathfrak{L}_{0}, \mathfrak{T}_{q}\right)$ and, as $\operatorname{id}_{\mathfrak{L}_{0}}$ and the operator of differentiation $\mathfrak{D}$ commute, inherit the derivatives from the presupposed differentiability of the mappings $\Xi_{L_{0}}$ with respect to the uniform topology.

Proof. (i) Note, that continuity of the mapping $(\Lambda, x) \mapsto \alpha_{(\Lambda, x)}(L)$ with respect to the locally convex space $\left(\mathfrak{L}, \mathfrak{T}_{q}\right)$ is equivalent to its continuity with respect to each of the topologizing seminorms $q_{\Delta}$.

Let the Borel subset $\Delta$ of $\mathbb{R}^{s+1}$ be arbitrary but fixed. We shall first consider the special point $(\mathbf{1}, 0) \in \mathrm{P}_{+}^{\uparrow}$ and restrict attention to an operator $L^{\prime} \in \mathfrak{L}_{0}$ having energymomentum transfer $\Gamma$ which, under transformations from a sufficiently small neighbourhood $\mathcal{N}^{\prime}$ of the neutral element $(\mathbf{1}, 0)$, stays bounded in a compact and convex subset $\widehat{\Gamma}$ of $\complement \bar{V}_{+}$. This means that all operators $\alpha_{(\Lambda, x)}\left(L^{\prime}\right) \in \mathfrak{L}_{0},(\Lambda, x) \in \mathcal{N}^{\prime}$, have energymomentum transfer in the common set $\widehat{\Gamma}$ and relation (2.6) of Proposition 2.6 applies to the differences $\alpha_{(\Lambda, x)}\left(L^{\prime}\right)-L^{\prime}$ yielding

$$
\begin{aligned}
& q_{\Delta}\left(\alpha_{(\Lambda, x)}\left(L^{\prime}\right)-L^{\prime}\right)^{2} \\
& =\left\|E(\Delta) \int_{\mathbb{R}^{s}} d^{s} y \alpha_{y}\left(\left(\alpha_{(\Lambda, x)}\left(L^{\prime}\right)-L^{\prime}\right)^{*}\left(\alpha_{(\Lambda, x)}\left(L^{\prime}\right)-L^{\prime}\right)\right) E(\Delta)\right\| \\
& \quad \leqslant N(\Delta, \widehat{\Gamma}) \int_{\mathbb{R}^{s}} d^{s} y\left\|\left[\alpha_{y}\left(\alpha_{(\Lambda, x)}\left(L^{\prime}\right)-L^{\prime}\right),\left(\alpha_{(\Lambda, x)}\left(L^{\prime}\right)-L^{\prime}\right)^{*}\right]\right\| .
\end{aligned}
$$

An estimate for the integrand on the right-hand side can be based on relation (2.2c), requiring suitable approximating nets of local operators for $\alpha_{(\Lambda, x)}\left(L^{\prime}\right)-L^{\prime}$. Given $R_{0}>$ 0 there exists a neighbourhood $\mathcal{N}^{\prime \prime}$ of $(\mathbf{1}, x)$ such that $\mathcal{N}^{\prime \prime} \mathcal{O}_{r} \subseteq \mathcal{O}_{2 r}$ for $r>R_{0}$, and if $\left\{L_{r}^{\prime} \in \mathfrak{A}\left(\mathcal{O}_{r}\right): r>0\right\}$ is an approximating net of local operators for $L^{\prime}$, then $\alpha_{(\Lambda, x)}\left(L_{r}^{\prime}\right) \in$ $\mathfrak{A}\left(\mathcal{O}_{2 r}\right)$ for any $r>R_{0}$ and $(\Lambda, x) \in \mathcal{N}^{\prime \prime}$. Now

$$
r^{k}\left\|\alpha_{(\Lambda, x)}\left(L^{\prime}\right)-\alpha_{(\Lambda, x)}\left(L_{r}^{\prime}\right)\right\|=r^{k}\left\|L^{\prime}-L_{r}^{\prime}\right\| \underset{r \rightarrow \infty}{\longrightarrow} 0
$$

holds for any $k \in \mathbb{N}$, so that the operators $\alpha_{(\Lambda, x)}\left(L_{r}^{\prime}\right)-L_{r}^{\prime} \in \mathfrak{A}\left(\mathcal{O}_{2 r}\right), r>R_{0}$, constitute the large radius part of approximating nets for each of $\alpha_{(\Lambda, x)}\left(L^{\prime}\right)-L^{\prime},(\Lambda, x) \in \mathcal{N}^{\prime \prime}$, subject to the bound

$$
\left\|\left(\alpha_{(\Lambda, x)}\left(L^{\prime}\right)-L^{\prime}\right)-\left(\alpha_{(\Lambda, x)}\left(L_{r}^{\prime}\right)-L_{r}^{\prime}\right)\right\| \leqslant\left\|L^{\prime}-L_{r}^{\prime}\right\|,
$$

which is independent of $(\Lambda, x) \in \mathcal{N}^{\prime \prime}$. Then, according to the remark following Definition 2.1, there exist approximating nets $\left\{L^{\prime}(\Lambda, x)_{r} \in \mathfrak{A}\left(\mathcal{O}_{r}\right): r>0\right\}$ for the almost local operators $\alpha_{(\Lambda, x)}\left(L^{\prime}\right)-L^{\prime}$ that fulfill the estimates $\left\|L^{\prime}(\Lambda, x)_{r}\right\| \leqslant\left\|\alpha_{(\Lambda, x)}\left(L^{\prime}\right)-L^{\prime}\right\|$ and, for $r>R_{0},\left\|\left(\alpha_{(\Lambda, x)}\left(L^{\prime}\right)-L^{\prime}\right)-L^{\prime}(\Lambda, x)_{2 r}\right\| \leqslant 2\left\|L^{\prime}-L_{r}^{\prime}\right\|$, where in view of (2.22) the second inequality amounts to $\left\|\left(\alpha_{(\Lambda, x)}\left(L^{\prime}\right)-L^{\prime}\right)-L^{\prime}(\Lambda, x)_{2 r}\right\| \leqslant 2 C_{k} r^{-k}$ for suitable $C_{k}>0$. Making use of relation (2.2c) in the same remark we arrive at

$$
\begin{array}{r}
\left\|\left[\alpha_{y}\left(\alpha_{(\Lambda, x)}\left(L^{\prime}\right)-L^{\prime}\right),\left(\alpha_{(\Lambda, x)}\left(L^{\prime}\right)-L^{\prime}\right)^{*}\right]\right\| \\
\leqslant 2\left\|\alpha_{(\Lambda, x)}\left(L^{\prime}\right)-L^{\prime}\right\|^{2} \chi_{<}(\boldsymbol{y})+8\left\|\alpha_{(\Lambda, x)}\left(L^{\prime}\right)-L^{\prime}\right\|\left\|L^{\prime}-L_{4^{-1}|\boldsymbol{y}|}^{\prime}\right\| \chi_{>}(\boldsymbol{y}) \\
\leqslant 8\left\|L^{\prime}\right\|^{2} \chi_{<}(\boldsymbol{y})+16\left\|L^{\prime}\right\| C_{k} 4^{k}|\boldsymbol{y}|^{-k} \chi_{>}(\boldsymbol{y})
\end{array}
$$


for any $\boldsymbol{y} \in \mathbb{R}^{s}$, where $\chi_{<}$and $\chi>$ denote the characteristic functions pertaining to the compact ball of radius $4 R_{0}$ in $\mathbb{R}^{s}$ and its complement, respectively. The above relation (2.23) holds for any $(\Lambda, x) \in \mathcal{N} \doteq \mathcal{N}^{\prime} \cap \mathcal{N}^{\prime \prime}$, and its right-hand side turns out to be an integrable majorizing function for the mapping

$$
\boldsymbol{y} \mapsto\left\|\left[\alpha_{y}\left(\alpha_{(\Lambda, x)}\left(L^{\prime}\right)-L^{\prime}\right),\left(\alpha_{(\Lambda, x)}\left(L^{\prime}\right)-L^{\prime}\right)^{*}\right]\right\|,
$$

irrespective of $(\Lambda, x) \in \mathcal{N}$, if $k \geqslant s+2$. Another consequence of (2.23) is that the function (2.24) converges pointwise to 0 on $\mathbb{R}^{s}$ in the limit $(\Lambda, x) \rightarrow(\mathbf{1}, 0)$ due to strong continuity of the automorphism group $\left\{\alpha_{(\Lambda, x)}:(\Lambda, x) \in \mathrm{P}_{+}^{\uparrow}\right\}$. Therefore we can apply Lebesgue's Dominated Convergence Theorem to the integral on the right-hand side of (2.21), evaluated for any sequence $\left\{\left(\Lambda_{n}, x_{n}\right)\right\}_{n \in \mathbb{N}} \subseteq \mathcal{N}$ approaching $(\mathbf{1}, 0)$ and infer

$$
\lim _{n \rightarrow \infty} q_{\Delta}\left(\alpha_{\left(\Lambda_{n}, x_{n}\right)}\left(L^{\prime}\right)-L^{\prime}\right)=0
$$

Since $\mathrm{P}_{+}^{\uparrow}$ as a topological space satisfies the first axiom of countability, this suffices to establish continuity of the mapping $(\Lambda, x) \mapsto \alpha_{(\Lambda, x)}\left(L^{\prime}\right)$ in $(\mathbf{1}, 0)$ with respect to the $q_{\Delta}$-topology.

An arbitrary operator $L \in \mathfrak{L}$ can be represented as $L=\sum_{i=1}^{N} A_{i} L_{i}^{\prime}$ where $L_{i}^{\prime} \in \mathfrak{L}_{0}$ comply with the above assumptions on $L^{\prime}$ and the operators $A_{i}$ belong to the quasi-local algebra $\mathfrak{A}$ for any $i=1, \ldots, N$. According to Lemma 2.12 we have

$$
\begin{aligned}
& 0 \leqslant q_{\Delta}\left(\alpha_{(\Lambda, x)}(L)-L\right) \\
& \leqslant \sum_{i=1}^{N}\left(q_{\Delta}\left(\alpha_{(\Lambda, x)}\left(A_{i}\right)\left(\alpha_{(\Lambda, x)}\left(L_{i}^{\prime}\right)-L_{i}^{\prime}\right)\right)+q_{\Delta}\left(\left(\alpha_{(\Lambda, x)}\left(A_{i}\right)-A_{i}\right) L_{i}^{\prime}\right)\right) \\
& \leqslant \sum_{i=1}^{N}\left(\left\|A_{i}\right\| q_{\Delta}\left(\alpha_{(\Lambda, x)}\left(L_{i}^{\prime}\right)-L_{i}^{\prime}\right)+\left\|\alpha_{(\Lambda, x)}\left(A_{i}\right)-A_{i}\right\| q_{\Delta}\left(L_{i}^{\prime}\right)\right),
\end{aligned}
$$

where the right-hand side vanishes in the limit $(\Lambda, x) \rightarrow(\mathbf{1}, 0)$ due to the preceding result and strong continuity of the group $\left\{\alpha_{(\Lambda, x)}:(\Lambda, x) \in \mathrm{P}_{+}^{\uparrow}\right\}$. Thus the mapping $(\Lambda, x) \mapsto \alpha_{(\Lambda, x)}(L)$ turns out to be continuous in $(\mathbf{1}, 0)$ with respect to $q_{\Delta}$ for arbitrary $L \in \mathfrak{L}$. The restriction to the specific point $(\mathbf{1}, 0) \in \mathrm{P}_{+}^{\uparrow}$ is inessential in the last step since for arbitrary $\left(\Lambda^{\prime}, x^{\prime}\right),\left(\Lambda_{0}, x_{0}\right) \in \mathrm{P}_{+}^{\uparrow}$ one has

$$
q_{\Delta}\left(\alpha_{\left(\Lambda^{\prime}, x^{\prime}\right)}(L)-\alpha_{\left(\Lambda_{0}, x_{0}\right)}(L)\right)=q_{\Delta}\left(\alpha_{\left(\Lambda^{\prime}, x^{\prime}\right)\left(\Lambda_{0}, x_{0}\right)^{-1}}\left(\alpha_{\left(\Lambda_{0}, x_{0}\right)}(L)\right)-\alpha_{\left(\Lambda_{0}, x_{0}\right)}(L)\right),
$$

explicitly showing that continuity of $(\Lambda, x) \mapsto \alpha_{(\Lambda, x)}(L)$ in $\left(\Lambda_{0}, x_{0}\right)$ is equivalent to continuity of $(\Lambda, x) \mapsto \alpha_{(\Lambda, x)}\left(\alpha_{\left(\Lambda_{0}, x_{0}\right)}(L)\right)$ in $(\mathbf{1}, 0)$ with respect to any of the seminorms $q_{\Delta}$, where $\alpha_{\left(\Lambda_{0}, x_{0}\right)}(L)$ belongs to $\mathfrak{L}$.

(ii) Continuity of a mapping with values in the locally convex space $\left(\mathfrak{C}, \mathfrak{T}_{p}\right)$ is equivalent to its continuity with respect to all seminorms $p_{\Delta}$. The problem at hand thus reduces to the one already solved in the first part, if one takes into account the shape of general elements of $\mathfrak{C}$ according to Definition 2.5 and Corollary 2.13.

(iii) According to Definition A.16 we have to show that for any vacuum annihilation operator $L_{0} \in \mathfrak{L}_{0}$ the mapping $(\Lambda, x) \mapsto \Xi_{L_{0}}(\Lambda, x)=\alpha_{(\Lambda, x)}\left(L_{0}\right)$ is differentiable in the 
locally convex space $\left(\mathfrak{L}_{0}, \mathfrak{T}_{q}\right)$ and has derivatives coinciding with those existing in the uniform topology by assumption.

Let $L_{0} \in \mathfrak{L}_{0}$ be given and consider the local chart $(\mathrm{U}, \phi)$ around $\left(\Lambda_{0}, x_{0}\right)=\phi^{-1}\left(\boldsymbol{t}_{0}\right)$. Due to the presupposed differentiability of the mapping $\Xi_{L_{0}}$ with respect to the uniform topology the corresponding residual term at $\left(\Lambda_{0}, x_{0}\right)$ with respect to $(U, \phi)$ is given by

$$
R\left[\Xi_{L_{0}} \circ \phi^{-1}, t_{0}\right](\boldsymbol{h})=\alpha_{\left(\Lambda_{\boldsymbol{h}}, x_{h}\right)}\left(L_{0}\right)-\alpha_{\left(\Lambda_{0}, x_{0}\right)}\left(L_{0}\right)-\mathfrak{D}_{\phi} \Xi_{L_{0}}\left(\Lambda_{0}, x_{0}\right) \boldsymbol{h},
$$

using the notation $\left(\Lambda_{\boldsymbol{h}^{\prime}}, x_{\boldsymbol{h}^{\prime}}\right) \doteq \phi^{-1}\left(\boldsymbol{t}_{0}+\boldsymbol{h}^{\prime}\right)$ for elements of $\mathrm{U}$, and satisfies the limit condition

$$
\lim _{\boldsymbol{h} \rightarrow \boldsymbol{0}}|\boldsymbol{h}|^{-1}\left\|R\left[\Xi_{L_{0}} \circ \phi^{-1}, \boldsymbol{t}_{0}\right](\boldsymbol{h})\right\|=0
$$

To prove the assertion it has to be shown that (2.25b) stays true when the norm is replaced by any of the seminorms $q_{\Delta}$. Now, according to the Mean Value Theorem A.7, we have for small $\boldsymbol{h}$

$$
\alpha_{\left(\Lambda_{h}, x_{h}\right)}\left(L_{0}\right)-\alpha_{\left(\Lambda_{0}, x_{0}\right)}\left(L_{0}\right)=\int_{0}^{1} d \vartheta \mathfrak{D}_{\phi} \Xi_{L_{0}}\left(\Lambda_{\vartheta \boldsymbol{h}}, x_{\vartheta \boldsymbol{h}}\right) \boldsymbol{h},
$$

where the integral is to be understood with respect to the norm topology of $\mathfrak{A}$. Thus the residual term $(2.25 \mathrm{a})$ can be re-written as

$$
\begin{aligned}
& R\left[\Xi_{L_{0}} \circ \phi^{-1}, \boldsymbol{t}_{0}\right](\boldsymbol{h})=\int_{0}^{1} d \vartheta\left(\mathfrak{D}_{\phi} \Xi_{L_{0}}\left(\Lambda_{\vartheta \boldsymbol{h}}, x_{\vartheta \boldsymbol{h}}\right)-\mathfrak{D}_{\phi} \Xi_{L_{0}}\left(\Lambda_{0}, x_{0}\right)\right) \boldsymbol{h} \\
& \quad=\sum_{i, j=1}^{d_{\mathrm{P}}} \int_{0}^{1} d \vartheta h_{i}\left(C_{i j}\left(\Lambda_{\vartheta \boldsymbol{h}}, x_{\vartheta \boldsymbol{h}}\right) \alpha_{\left(\Lambda_{\vartheta \boldsymbol{h}}, x_{\vartheta \boldsymbol{h}}\right)}\left(\delta^{j}\left(L_{0}\right)\right)-C_{i j}\left(\Lambda_{0}, x_{0}\right) \alpha_{\left(\Lambda_{0}, x_{0}\right)}\left(\delta^{j}\left(L_{0}\right)\right)\right),
\end{aligned}
$$

where in the last equation (A.20a) is used to represent the linear operator $\mathfrak{D}_{\phi} \Xi_{L_{0}}$ in terms of partial derivatives of $\Xi_{L_{0}}$ which can be expressed by means of analytic functions $C_{i j}$ on $\mathrm{U}$ and Poincaré transformed derivatives $\delta^{j}\left(L_{0}\right)$ of $L_{0}\left(d_{\mathrm{P}}\right.$ is the dimension of the Poincaré group). As a consequence of the first statement of this proposition, the integrand on the right-hand side is continuous with respect to all seminorms $q_{\Delta}$, so that the integral exists in the complete locally convex space $\left(\overline{\mathfrak{L}}, \overline{\mathfrak{T}}_{q}\right)$. By [26, II.6.2 and 5.4] this leads to the following estimate for the residual term

$$
\begin{aligned}
& |\boldsymbol{h}|^{-1} q_{\Delta}\left(R\left[\Xi_{L_{0}} \circ \phi^{-1}, \boldsymbol{t}_{0}\right](\boldsymbol{h})\right) \\
& \leqslant \sum_{i, j=1}^{d_{\mathrm{P}}} \int_{0}^{1} d \vartheta \frac{\left|h_{i}\right|}{|\boldsymbol{h}|} q_{\Delta}\left(C_{i j}\left(\Lambda_{\vartheta \boldsymbol{h}}, x_{\vartheta \boldsymbol{h}}\right) \alpha_{\left(\Lambda_{\vartheta \boldsymbol{h}}, x_{\vartheta \boldsymbol{h}}\right)}\left(\delta^{j}\left(L_{0}\right)\right)-C_{i j}\left(\Lambda_{0}, x_{0}\right) \alpha_{\left(\Lambda_{0}, x_{0}\right)}\left(\delta^{j}\left(L_{0}\right)\right)\right) \\
& \quad \leqslant \sum_{i, j=1}^{d_{\mathrm{P}}} \max _{0 \leqslant \vartheta \leqslant 1} q_{\Delta}\left(C_{i j}\left(\Lambda_{\vartheta \boldsymbol{h}}, x_{\vartheta \boldsymbol{h}}\right) \alpha_{\left(\Lambda_{\vartheta \boldsymbol{h}}, x_{\vartheta \boldsymbol{h}}\right)}\left(\delta^{j}\left(L_{0}\right)\right)-C_{i j}\left(\Lambda_{0}, x_{0}\right) \alpha_{\left(\Lambda_{0}, x_{0}\right)}\left(\delta^{j}\left(L_{0}\right)\right)\right),
\end{aligned}
$$

where evidently the right-hand side vanishes in the limit $\boldsymbol{h} \rightarrow \boldsymbol{0}$. Thus condition (A.1b) for differentiability of mappings with values in a locally convex space is fulfilled, and according to the counterpart (2.25a) of (A.1a) the derivatives of $\Xi_{L_{0}}$ with respect to both the uniform and locally convex topologies on $\mathfrak{L}_{0}$ coincide. 


\subsubsection{Integrability}

Having established Proposition 2.16 on continuity of the mappings $(\Lambda, x) \mapsto \alpha_{(\Lambda, x)}(L)$ and $(\Lambda, x) \mapsto \alpha_{(\Lambda, x)}(C)$ for given $L \in \mathfrak{L}$ and $C \in \mathfrak{C}$, it turns out to be possible to construct new elements of $\left(\mathfrak{L}_{0}, \mathfrak{T}_{q}\right),\left(\mathfrak{A}_{\mathfrak{L}}, \overline{\mathfrak{T}}_{q}^{u}\right)$ and $\left(\mathfrak{A}_{\mathfrak{C}}, \overline{\mathfrak{T}}_{p}^{u}\right)$ through integration with respect to the Haar measure on $\mathrm{P}_{+}^{\uparrow}$.

Lemma 2.17. Let the function $F \in L^{1}\left(\mathrm{P}_{+}^{\uparrow}, d \mu(\Lambda, x)\right)$ have compact support $\mathrm{S}$.

(i) For any $L_{0} \in \mathfrak{L}_{0}$ the operator

$$
\alpha_{F}\left(L_{0}\right) \doteq \int d \mu(\Lambda, x) F(\Lambda, x) \alpha_{(\Lambda, x)}\left(L_{0}\right)
$$

belongs to $\mathfrak{L}_{0}$, too.

(ii) If $L \in \mathfrak{L}$ and $C \in \mathfrak{C}$, then

$$
\begin{aligned}
& \alpha_{F}(L) \doteq \int d \mu(\Lambda, x) F(\Lambda, x) \alpha_{(\Lambda, x)}(L), \\
& \alpha_{F}(C) \doteq \int d \mu(\Lambda, x) F(\Lambda, x) \alpha_{(\Lambda, x)}(C)
\end{aligned}
$$

exist as integrals in the complete locally convex spaces $\left(\mathfrak{A}_{\mathfrak{L}}, \overline{\mathfrak{T}}_{q}^{u}\right)$ and $\left(\mathfrak{A}_{\mathfrak{C}}, \overline{\mathfrak{T}}_{p}^{u}\right)$, respectively, and for any bounded Borel set $\Delta$ there hold the estimates

$$
\begin{aligned}
& q_{\Delta}\left(\alpha_{F}(L)\right) \leqslant\|F\|_{1} \sup _{(\Lambda, x) \in \mathrm{S}} q_{\Delta}\left(\alpha_{(\Lambda, x)}(L)\right), \\
& p_{\Delta}\left(\alpha_{F}(C)\right) \leqslant\|F\|_{1} \sup _{(\Lambda, x) \in \mathrm{S}} p_{\Delta}\left(\alpha_{(\Lambda, x)}(C)\right) .
\end{aligned}
$$

Proof. (i) By assumption $(\Lambda, x) \mapsto|F(\Lambda, x)|\left\|\alpha_{(\Lambda, x)}\left(L_{0}\right)\right\|=|F(\Lambda, x)|\left\|L_{0}\right\|[54$, Corollary I.5.4] is an integrable majorizing function for the integrand of (2.26), so $\alpha_{F}\left(L_{0}\right)$ exists as a Bochner integral in $\mathfrak{A}$. The same holds true for the integrals constructed by use of an approximating net $\left\{L_{0, r} \in \mathfrak{A}\left(\mathcal{O}_{r}\right): r>0\right\}$ for the almost local operator $L_{0} \in \mathfrak{L}_{0}$ :

$$
\alpha_{F}\left(L_{0, r}\right) \doteq \int_{\mathrm{S}} d \mu(\Lambda, x) F(\Lambda, x) \alpha_{(\Lambda, x)}\left(L_{0, r}\right)
$$

Due to compactness of $\mathrm{S}$, these operators belong to the local algebras $\mathfrak{A}\left(\mathcal{O}_{r(\mathrm{~S})}\right)$ pertaining to standard diamonds in $\mathbb{R}^{s+1}$ which have each an $s$-dimensional basis of radius $r(\mathrm{~S}) \doteq a(\mathrm{~S}) r+b(\mathrm{~S})$ where $a(\mathrm{~S})$ and $b(\mathrm{~S})$ are suitable positive constants. Now,

$$
\alpha_{F}\left(L_{0}\right)-\alpha_{F}\left(L_{0, r}\right)=\int_{\mathrm{S}} d \mu(\Lambda, x) F(\Lambda, x)\left(\alpha_{(\Lambda, x)}\left(L_{0}\right)-\alpha_{(\Lambda, x)}\left(L_{0, r}\right)\right),
$$

so that we arrive at the estimate $(\mu(\mathrm{S})$ is the measure of the compact set $\mathrm{S})$

$$
r(\mathrm{~S})^{k}\left\|\alpha_{F}\left(L_{0}\right)-\alpha_{F}\left(L_{0, r}\right)\right\| \leqslant \mu(\mathrm{S})\|F\|_{1}(a(\mathrm{~S}) r+b(\mathrm{~S}))^{k}\left\|L_{0}-L_{0, r}\right\|
$$


which holds for any $k \in \mathbb{N}$. Due to almost locality of $L_{0}$, the right-hand side vanishes in the limit of large $r$, so that the operator $\alpha_{F}\left(L_{0}\right)$ itself turns out to be almost local: $\alpha_{F}\left(L_{0}\right) \in \mathfrak{A}_{\mathcal{S}}$ with approximating net $\left\{\alpha_{F}\left(L_{0, r}\right) \in \mathfrak{A}\left(\mathcal{O}_{r(\mathrm{~S})}\right): r>0\right\}$.

Let $\Gamma \subseteq \complement \bar{V}_{+}$denote the energy-momentum transfer of the vacuum annihilation operator $L_{0}$, then, by the Fubini Theorem [26, II.16.3], the following equation is valid for any $g \in L^{1}\left(\mathbb{R}^{s+1}, d^{s+1} y\right)$

$$
\int_{\mathbb{R}^{s+1}} d^{s+1} y g(y) \alpha_{y}\left(\alpha_{F}\left(L_{0}\right)\right)=\int_{S} d \mu(\Lambda, x) F(\Lambda, x) \int_{\mathbb{R}^{s+1}} d^{s+1} y g(y) \alpha_{y}\left(\alpha_{(\Lambda, x)}\left(L_{0}\right)\right) .
$$

In the special case supp $\tilde{g} \subseteq \bigcap_{(\Lambda, x) \in S} \complement(\Lambda \Gamma), \tilde{g}$ the Fourier transform of $g$, the inner integrals on the right-hand side vanish for any $(\Lambda, x) \in \mathrm{S}$ so that we infer

$$
\int_{\mathbb{R}^{s+1}} d^{s+1} y g(y) \alpha_{y}\left(\alpha_{F}\left(L_{0}\right)\right)=0
$$

which shows that the energy-momentum transfer of $\alpha_{F}\left(L_{0}\right)$ is contained in the compact subset $\bigcup_{(\Lambda, x) \in S} \Lambda \Gamma$ of $C \bar{V}_{+}$. Therefore $\alpha_{F}\left(L_{0}\right)$ is indeed a vacuum annihilation operator from $\mathfrak{A}_{\text {ann }}$.

Finally, infinite differentiability with respect to the uniform topology of the mapping

$$
(\Lambda, x) \mapsto \Xi_{\alpha_{F}\left(L_{0}\right)}(\Lambda, x)=\alpha_{(\Lambda, x)}\left(\alpha_{F}\left(L_{0}\right)\right)
$$

has to be established. By assumption $L_{0}$ is infinitely often differentiable with respect to the Poincaré group, which implies that likewise all the operators $\alpha_{(\Lambda, x)}\left(L_{0}\right)$ belong to $\mathcal{D}^{(\infty)}(\mathfrak{A})$ for any $(\Lambda, x) \in \mathrm{P}_{+}^{\uparrow}$. Their residual terms at $(\mathbf{1}, 0)=\phi_{0}^{-1}(\boldsymbol{0})$ with respect to the canonical coordinates $\left(\mathrm{U}_{0}, \phi_{0}\right)$ of the first kind, as introduced in [55, Section 2.10], can, using the notation $\left(\Lambda_{\boldsymbol{h}^{\prime}}, x_{\boldsymbol{h}^{\prime}}\right) \doteq \phi_{0}^{-1}\left(\boldsymbol{h}^{\prime}\right)$ for the transformations in $\mathrm{U}_{0}$, be expressed by

$$
\begin{aligned}
R\left[\Xi_{\alpha_{(\Lambda, x)}\left(L_{0}\right)} \circ \phi_{0}^{-1}, \boldsymbol{o}\right] & (\boldsymbol{h}) \\
= & \alpha_{\left(\Lambda_{\boldsymbol{h}}, x_{\boldsymbol{h}}\right)}\left(\alpha_{(\Lambda, x)}\left(L_{0}\right)\right)-\alpha_{(\Lambda, x)}\left(L_{0}\right)-\mathfrak{D}_{\phi_{0}} \Xi_{\alpha_{(\Lambda, x)}\left(L_{0}\right)}(\mathbf{1}, 0) \boldsymbol{h} \\
\quad & \int_{0}^{1} d \vartheta\left(\mathfrak{D}_{\phi_{0}} \Xi_{\alpha_{(\Lambda, x)}\left(L_{0}\right)}\left(\Lambda_{\vartheta \boldsymbol{h}}, x_{\vartheta \boldsymbol{h}}\right)-\mathfrak{D}_{\phi_{0}} \Xi_{\alpha_{(\Lambda, x)}\left(L_{0}\right)}(\mathbf{1}, 0)\right) \boldsymbol{h},
\end{aligned}
$$

where the last equation stems from an application of the Mean Value Theorem A.7, which holds true for small $\boldsymbol{h}$. By Proposition A.11 the term $\mathfrak{D}_{\phi_{0}} \Xi_{\alpha_{(\Lambda, x)}\left(L_{0}\right)}(\mathbf{1}, 0) \boldsymbol{h}$ on the second line is continuous in $(\Lambda, x)$, so that it is possible to multiply (2.29) with the function $F(\Lambda, x)$ and subsequently integrate over its compact support S. Taking into account that each of the automorphisms $\alpha_{\left(\Lambda_{h}, x_{h}\right)}$ is uniformly continuous, thus commuting with Bochner integrals, this yields

$$
\begin{aligned}
& \int_{\mathrm{S}} d \mu(\Lambda, x) F(\Lambda, x) R\left[\Xi_{\alpha_{(\Lambda, x)}\left(L_{0}\right)} \circ \phi_{0}^{-1}, \boldsymbol{0}\right](\boldsymbol{h}) \\
& \quad=\alpha_{\left(\Lambda_{h}, x_{h}\right)}\left(\alpha_{F}\left(L_{0}\right)\right)-\alpha_{F}\left(L_{0}\right)-\int_{\mathrm{S}} d \mu(\Lambda, x) F(\Lambda, x) \mathfrak{D}_{\phi_{0}} \Xi_{\alpha_{(\Lambda, x)}\left(L_{0}\right)}(\mathbf{1}, 0) \boldsymbol{h},
\end{aligned}
$$


which has the shape of a residual term for $\Xi_{\alpha_{F}\left(L_{0}\right)}$ at $(\mathbf{1}, 0)$. Now, the operator-norm of $\mathfrak{D}_{\phi_{0}} \Xi_{\alpha_{(\Lambda, x)}\left(L_{0}\right)}\left(\Lambda_{\vartheta \boldsymbol{h}}, x_{\vartheta \boldsymbol{h}}\right)$ can be estimated according to (A.17) by

$$
\left\|\mathfrak{D}_{\phi_{0}} \Xi_{\alpha_{(\Lambda, x)}\left(L_{0}\right)}\left(\Lambda_{\vartheta \boldsymbol{h}}, x_{\vartheta \boldsymbol{h}}\right)\right\| \leqslant\left\|\mathfrak{D}_{\phi_{0}} \Xi_{L_{0}}(\mathbf{1})\right\|\|\mathbf{N}(\Lambda, x)\|\left\|\mathbf{M}^{\phi_{0}}\left(\Lambda_{\vartheta \boldsymbol{h}}, x_{\vartheta \boldsymbol{h}}\right)\right\|,
$$

which, due to continuity of $(\Lambda, x) \mapsto \mathbf{N}(\Lambda, x)$ and $\vartheta \mapsto \mathbf{M}^{\phi_{0}}\left(\Lambda_{\vartheta \boldsymbol{h}}, x_{\vartheta \boldsymbol{h}}\right)$ with respect to the operator-norm topology, is majorized on the compact set $\mathrm{S} \times[0,1]$ by a constant $K(\mathrm{~S})$. As a consequence of the last equation in (2.29) we then get for any $(\Lambda, x) \in \mathrm{S}$ and small $\boldsymbol{h}$ the bound

$$
|\boldsymbol{h}|^{-1}\left\|F(\Lambda, x) R\left[\Xi_{\alpha_{(\Lambda, x)}\left(L_{0}\right)} \circ \phi_{0}^{-1}, \boldsymbol{0}\right](\boldsymbol{h})\right\| \leqslant 2 K(\mathrm{~S})|F(\Lambda, x)|,
$$

which is integrable over $S$ by assumption; restricting furthermore attention to sequences $\left\{\boldsymbol{h}_{n}\right\}_{n \in \mathbb{N}}$ converging to $\boldsymbol{0}$, we see that the left-hand side of (2.31) converges pointwise to 0 . With this information at hand it is possible to apply Lebesgue's Dominated Convergence Theorem [26, II.5.6] to the left-hand side of (2.30) to get

$$
\lim _{n \rightarrow \infty}\left|\boldsymbol{h}_{n}\right|^{-1} \int_{\mathrm{S}} d \mu(\Lambda, x) F(\Lambda, x) R\left[\Xi_{\alpha_{(\Lambda, x)}\left(L_{0}\right)} \circ \phi_{0}^{-1}, \boldsymbol{0}\right]\left(\boldsymbol{h}_{n}\right)=0,
$$

which is sufficient to establish condition (A.1b) for differentiability of the mapping $\Xi_{\alpha_{F}\left(L_{0}\right)}$ at $(\mathbf{1}, 0)$. The linear operator defining the corresponding derivative is according to the right-hand side of (2.30) in connection with (A.17) given by

$$
\begin{aligned}
\mathfrak{D}_{\phi_{0}} \Xi_{\alpha_{F}\left(L_{0}\right)}(\mathbf{1}, 0) \boldsymbol{h} & =\int_{\mathrm{S}} d \mu(\Lambda, x) F(\Lambda, x) \mathfrak{D}_{\phi_{0}} \Xi_{\alpha_{(\Lambda, x)}\left(L_{0}\right)}(\mathbf{1}, 0) \boldsymbol{h} \\
= & \int_{\mathrm{S}} d \mu(\Lambda, x) F(\Lambda, x) \alpha_{(\Lambda, x)} \circ \mathfrak{D}_{\phi_{0}} \Xi_{L_{0}}(\mathbf{1}, 0) \circ \mathbf{N}(\Lambda, x) \boldsymbol{h} \\
& =\sum_{i, j=1}^{d_{\mathrm{P}}} h_{i} \int_{\mathrm{S}} d \mu(\Lambda, x) F_{j i}(\Lambda, x) \alpha_{(\Lambda, x)}\left(\delta^{j}\left(L_{0}\right)\right)=\sum_{i, j=1}^{d_{\mathrm{P}}} h_{i} \alpha_{F_{j i}}\left(\delta^{j}\left(L_{0}\right)\right),
\end{aligned}
$$

where $F_{j i}(\Lambda, x) \doteq F(\Lambda, x) \mathbf{N}_{j i}(\Lambda, x)$ are functions from $L^{1}\left(\mathrm{P}_{+}^{\uparrow}, d \mu(\Lambda, x)\right)$ with compact support $S$. Since $\mathfrak{L}_{0}$ is invariant under differentiation we conclude from the first two paragraphs of the present proof and the above considerations that the partial derivatives

$$
\delta^{i}\left(\alpha_{F}\left(L_{0}\right)\right)=\sum_{j=1}^{d_{\mathrm{P}}} \alpha_{F_{j i}}\left(\delta^{j}\left(L_{0}\right)\right)
$$

are again almost local vacuum annihilation operators which belong to $\mathcal{D}^{(1)}(\mathfrak{A})$. Thus by induction, repeatedly using these methods, $\alpha_{F}\left(L_{0}\right)$ is seen to be an element of $\mathcal{D}^{(\infty)}(\mathfrak{A})$ with almost local derivatives of any order, i. e. $\alpha_{F}\left(L_{0}\right) \in \mathfrak{L}_{0}$.

(ii) By Proposition 2.16 the mappings $(\Lambda, x) \mapsto \alpha_{(\Lambda, x)}(L)$ and $(\Lambda, x) \mapsto \alpha_{(\Lambda, x)}(C)$ are continuous with respect to the uniform topology and all the $q_{\Delta^{-}}$and $p_{\Delta^{-}}$-topologies, respectively, staying bounded on the compact set $S$. This implies their measurability in the locally convex spaces $\left(\mathfrak{A}_{\mathfrak{L}}, \overline{\mathfrak{T}}_{q}^{u}\right)$ and $\left(\mathfrak{A}_{\mathfrak{C}}, \overline{\mathfrak{T}}_{p}^{u}\right)$ together with the fact that their product with the integrable function $f$ is majorized in each of the norm and seminorm topologies by a multiple of $|F|$. As a consequence the integrals $\alpha_{F}(L)$ and $\alpha_{F}(C)$ exist in the complete locally convex spaces $\left(\mathfrak{A}_{\mathfrak{L}}, \overline{\mathfrak{T}}_{q}^{u}\right)$ and $\left(\mathfrak{A}_{\mathfrak{C}}, \overline{\mathfrak{T}}_{p}^{u}\right)$, respectively, and (2.28) is an immediate upshot [26, II.6.2 and 5.4]. 
There exists a version of the second part of the above lemma for functions on $\mathbb{R}^{s+1}$ that are Lebesgue-integrable but no longer have to be compactly supported.

Lemma 2.18. Let $L \in \mathfrak{L}$ and let $g \in L^{1}\left(\mathbb{R}^{s+1}, d^{s+1} x\right)$. Then

$$
\alpha_{g}(L) \doteq \int_{\mathbb{R}^{s+1}} d^{s+1} x g(x) \alpha_{x}(L)
$$

is an operator in $\left(\mathfrak{A}_{\mathfrak{L}}, \overline{\mathfrak{T}}_{q}^{u}\right)$, satisfying the estimates

$$
q_{\Delta}\left(\alpha_{g}(L)\right) \leqslant\|g\|_{1} q_{\Delta}(L)
$$

for any bounded Borel set $\Delta$. The energy-momentum transfer of $\alpha_{g}(L)$ is contained in supp $\tilde{g}$, the support of the Fourier transform $\tilde{g}$ of $g$.

Proof. By translation invariance of the norm $\|$. $\|$ as well as of the seminorms $q_{\Delta}$ (cf. Lemma 2.15) the (measurable) integrand on the right-hand side of (2.33) is majorized by the functions $x \mapsto|g(x)|\|L\|$ and $x \mapsto|g(x)| q_{\Delta}(L)$ for any bounded Borel set $\Delta$. These are Lebesgue-integrable and therefore $\alpha_{g}(L)$ exists as a unique element of $\left(\mathfrak{A}_{\mathfrak{L}}, \overline{\mathfrak{T}}_{q}^{u}\right)$, satisfying the claimed estimates (2.34).

Next, we consider an arbitrary function $h \in L^{1}\left(\mathbb{R}^{s+1}, d^{s+1} x\right)$. By Fubini's Theorem [26, II.16.3] and translation invariance of Lebesgue measure

$$
\begin{gathered}
\int_{\mathbb{R}^{s+1}} d^{s+1} y h(y) \alpha_{y}\left(\alpha_{g}(L)\right)=\int_{\mathbb{R}^{s+1}} d^{s+1} y h(y) \alpha_{y}\left(\int_{\mathbb{R}^{s+1}} d^{s+1} x g(x) \alpha_{x}(L)\right) \\
=\int_{\mathbb{R}^{s+1}} d^{s+1} y \int_{\mathbb{R}^{s+1}} d^{s+1} x h(y) g(x) \alpha_{x+y}(L) \\
=\int_{\mathbb{R}^{s+1}} d^{s+1} x\left(\int_{\mathbb{R}^{s+1}} d^{s+1} y h(y) g(x-y)\right) \alpha_{x}(L),
\end{gathered}
$$

where the term in brackets on the right-hand side of the last equation is the convolution product $h * g$ of $h$ and $g$. Its Fourier transform $\widetilde{h * g}$ is given by $\widetilde{h * g}(p)=$ $(2 \pi)^{(s+1) / 2} \tilde{h}(p) \tilde{g}(p)$ (cf. [39, Theorem VI.(21.41)]), so that this function vanishes if $\tilde{h}$ and $\tilde{g}$ have disjoint supports. Therefore supp $\tilde{h} \cap \operatorname{supp} \tilde{g}=\emptyset$ entails

$$
\int_{\mathbb{R}^{s+1}} d^{s+1} y h(y) \alpha_{y}\left(\alpha_{g}(L)\right)=0,
$$

and this shows that the Fourier transform of $y \mapsto \alpha_{y}\left(\alpha_{g}(L)\right)$ has support in supp $\tilde{g}$, which henceforth contains the energy-momentum transfer of $\alpha_{g}(L)$.

\subsubsection{Decay Property}

Eventually we are able to establish a property of rapid decay with respect to the seminorms $q_{\Delta}$ for commutators of elements of $\mathfrak{L}$ which are almost local.

Lemma 2.19. Let $L_{1}$ and $L_{2}$ belong to $\mathfrak{L}_{0}$ and let $A_{1}, A_{2} \in \mathfrak{A}$ be almost local. Then for any bounded Borel subset $\Delta$ of $\mathbb{R}^{s+1}$

$$
\mathbb{R}^{s} \ni \boldsymbol{x} \mapsto q_{\Delta}\left(\left[\alpha_{x}\left(A_{1} L_{1}\right), A_{2} L_{2}\right]\right)
$$

decreases with $|\boldsymbol{x}| \rightarrow \infty$ faster than any power of $|\boldsymbol{x}|^{-1}$. 
Proof. First we consider the special case of two elements $L_{a}$ and $L_{b}$ in $\mathfrak{L}_{0}$ having energy-momentum transfer in compact and convex subsets $\Gamma_{a}$ and $\Gamma_{b}$ of $\complement \bar{V}_{+}$, respectively, with the additional property that $\Gamma_{a, b} \doteq\left(\Gamma_{a}+\Gamma_{b}\right)-\Gamma_{a}$ and $\Gamma_{b, a} \doteq\left(\Gamma_{a}+\Gamma_{b}\right)-\Gamma_{b}$ lie in the complement of $\bar{V}_{+}$, too. According to the Lemmas 2.14 and 2.12

$$
\begin{aligned}
& q_{\Delta}\left(\left[\alpha_{x}\left(L_{a}\right), L_{b}\right]\right)^{2}=p_{\Delta}\left(\left[\alpha_{x}\left(L_{a}\right), L_{b}\right]^{*}\left[\alpha_{x}\left(L_{a}\right), L_{b}\right]\right) \\
& \quad \leqslant q_{\Delta}\left(L_{b}\right) q_{\Delta}\left(\alpha_{x}\left(L_{a}\right)^{*}\left[\alpha_{x}\left(L_{a}\right), L_{b}\right]\right)+q_{\Delta}\left(L_{a}\right) q_{\Delta}\left(L_{b}{ }^{*}\left[\alpha_{x}\left(L_{a}\right), L_{b}\right]\right),
\end{aligned}
$$

and we are left with the task to investigate for large $|\boldsymbol{x}|$ the behaviour of the functions $q_{\Delta}\left(\alpha_{x}\left(L_{a}\right)^{*}\left[\alpha_{x}\left(L_{a}\right), L_{b}\right]\right)$ and $q_{\Delta}\left(L_{b}{ }^{*}\left[\alpha_{x}\left(L_{a}\right), L_{b}\right]\right)$. Since the arguments of both terms belong to $\mathfrak{L}_{0}$, having energy-momentum transfer in the compact and convex subsets $\Gamma_{a, b}$ and $\Gamma_{b, a}$ of $\complement \bar{V}_{+}$, we can apply (2.6) of Proposition 2.6 in connection with (2.17a) to get the estimate (for the second term)

$$
\begin{aligned}
& |\boldsymbol{x}|^{2 k} q_{\Delta}\left(L_{b}{ }^{*}\left[\alpha_{x}\left(L_{a}\right), L_{b}\right]\right)^{2} \\
& \quad \leqslant N\left(\Delta, \Gamma_{b, a}\right) \int_{\mathbb{R}^{s}} d^{s} y|\boldsymbol{x}|^{2 k}\left\|\left[\alpha_{y}\left(L_{b}{ }^{*}\left[\alpha_{x}\left(L_{a}\right), L_{b}\right]\right),\left(L_{b}{ }^{*}\left[\alpha_{x}\left(L_{a}\right), L_{b}\right]\right)^{*}\right]\right\| .
\end{aligned}
$$

Let $\left\{L_{a, r} \in \mathfrak{A}\left(\mathcal{O}_{r}\right): r>0\right\}$ and $\left\{L_{b, r} \in \mathfrak{A}\left(\mathcal{O}_{r}\right): r>0\right\}$ be approximating nets for $L_{a}$ and $L_{b}$, respectively, satisfying $\left\|L_{a, r}\right\| \leqslant\left\|L_{a}\right\|$ and $\left\|L_{b, r}\right\| \leqslant\left\|L_{b}\right\|$. Then the elements

$$
L_{b, r}{ }^{*}\left[\alpha_{x}\left(L_{a, r}\right), L_{b, r}\right] \in \mathfrak{A}\left(\mathcal{O}_{r}+\boldsymbol{x}\right) \subseteq \mathfrak{A}\left(\mathcal{O}_{r+|x|}\right)
$$

constitute the large radius part of approximating nets for the almost local operators $L_{b}{ }^{*}\left[\alpha_{x}\left(L_{a}\right), L_{b}\right], \boldsymbol{x} \in \mathbb{R}^{s}$, subject to the estimate

$$
\begin{aligned}
\| L_{b}{ }^{*}\left[\alpha_{x}\left(L_{a}\right), L_{b}\right]-L_{b, r} & {\left[\alpha_{x}\left(L_{a, r}\right), L_{b, r}\right] \| } \\
\leqslant & \leqslant\left\|L_{a}\right\|\left\|L_{b}\right\|\left\|L_{b}-L_{b, r}\right\|+2\left\|L_{b}\right\|^{2}\left\|L_{a}-L_{a, r}\right\| \leqslant C_{l} r^{-l}
\end{aligned}
$$

for any $l \in \mathbb{N}$ with suitable $C_{l}>0$. Now, as suggested by the remark following Definition 2.1, there exist approximating nets $\left\{L(a, b ; \boldsymbol{x})_{r} \in \mathfrak{A}\left(\mathcal{O}_{r}\right): r>0\right\}, \boldsymbol{x} \in \mathbb{R}^{s}$, with $\left\|L(a, b ; \boldsymbol{x})_{r}\right\| \leqslant\left\|L_{b}{ }^{*}\left[\alpha_{\boldsymbol{x}}\left(L_{a}\right), L_{b}\right]\right\|$ and $\left\|L_{b}{ }^{*}\left[\alpha_{x}\left(L_{a}\right), L_{b}\right]-L(a, b ; \boldsymbol{x})_{r+\mid \boldsymbol{x} \|}\right\| \leqslant 2 C_{l} r^{-l}$, so that, according to $(2.2 \mathrm{c})$, the integrand of (2.36) is bounded by

$$
\begin{aligned}
|\boldsymbol{x}|^{2 k}\left\|\left[\alpha_{\boldsymbol{y}}\left(L_{b}{ }^{*}\left[\alpha_{\boldsymbol{x}}\left(L_{a}\right), L_{b}\right]\right),\left(L_{b}{ }^{*}\left[\alpha_{x}\left(L_{a}\right), L_{b}\right]\right)^{*}\right]\right\| \\
\leqslant|\boldsymbol{x}|^{2 k} 4\left\|L_{b}{ }^{*}\left[\alpha_{\boldsymbol{x}}\left(L_{a}\right), L_{b}\right]\right\| \| L_{b}{ }^{*}\left[\alpha_{x}\left(L_{a}\right), L_{b}\right]-L(a, b ; \boldsymbol{x})_{2^{-1}|\boldsymbol{y}| \|} \\
\leqslant \begin{cases}8|\boldsymbol{x}|^{2 k}\left\|L_{b}\right\|^{2}\left\|\left[\alpha_{x}\left(L_{a}\right), L_{b}\right]\right\|^{2} & ,|\boldsymbol{y}| \leqslant 2(|\boldsymbol{x}|+1), \\
8\left\|L_{b}\right\||\boldsymbol{x}|^{2 k}\left\|\left[\alpha_{x}\left(L_{a}\right), L_{b}\right]\right\| C_{l}\left(2^{-1}|\boldsymbol{y}|-|\boldsymbol{x}|\right)^{-l} & ,|\boldsymbol{y}|>2(|\boldsymbol{x}|+1),\end{cases}
\end{aligned}
$$

which implies

$$
\begin{aligned}
& |\boldsymbol{x}|^{2 k} q_{\Delta}\left(L_{b}{ }^{*}\left[\alpha_{\boldsymbol{x}}\left(L_{a}\right), L_{b}\right]\right)^{2} \\
& \leqslant N\left(\Delta, \Gamma_{b, a}\right)\left[8\left\|L_{b}\right\|^{2}|\boldsymbol{x}|^{2 k}\left\|\left[\alpha_{\boldsymbol{x}}\left(L_{a}\right), L_{b}\right]\right\|^{2} \int_{|\boldsymbol{y}| \leqslant 2(|\boldsymbol{x}|+1)} d^{s} y\right. \\
& \left.\quad+8 C_{l}\left\|L_{b}\right\||\boldsymbol{x}|^{2 k}\left\|\left[\alpha_{\boldsymbol{x}}\left(L_{a}\right), L_{b}\right]\right\| \int_{|\boldsymbol{y}|>2(|\boldsymbol{x}|+1)} d^{s} y\left(2^{-1}|\boldsymbol{y}|-|\boldsymbol{x}|\right)^{-l}\right] .
\end{aligned}
$$


Evaluation of the integrals on the right-hand side yields (for $l \geqslant s+2$ ) polynomials of degree $s$ in $|\boldsymbol{x}|$, so that, due to the decay properties of $\boldsymbol{x} \mapsto\left\|\left[\alpha_{x}\left(L_{a}\right), L_{b}\right]\right\|$, there exists a uniform bound

$$
|\boldsymbol{x}|^{k} q_{\Delta}\left(L_{b}{ }^{*}\left[\alpha_{x}\left(L_{a}\right), L_{b}\right]\right)^{2} \leqslant M, \quad \boldsymbol{x} \in \mathbb{R}^{s} .
$$

The same reasoning applies to the term $q_{\Delta}\left(\alpha_{x}\left(L_{a}\right)^{*}\left[\alpha_{x}\left(L_{a}\right), L_{b}\right]\right)$, thus establishing the asserted rapid decrease for the mapping $\boldsymbol{x} \mapsto q_{\Delta}\left(\left[\alpha_{x}\left(L_{a}\right), L_{b}\right]\right)$, according to relation (2.35).

In the general case of almost local elements $A_{1}, A_{2} \in \mathfrak{A}$ and $L_{1}, L_{2} \in \mathfrak{L}_{0}$ one has, by Lemma 2.12

$$
\begin{aligned}
& q_{\Delta}\left(\left[\alpha_{x}\left(A_{1} L_{1}\right), A_{2} L_{2}\right]\right) \\
& \begin{array}{l}
\leqslant\left\|A_{1}\right\|\left\|\left[\alpha_{x}\left(L_{1}\right), A_{2}\right]\right\| \\
\quad q_{\Delta}\left(L_{2}\right)+\left\|A_{1}\right\|\left\|A_{2}\right\| q_{\Delta}\left(\left[\alpha_{x}\left(L_{1}\right), L_{2}\right]\right) \\
+\left\|\left[\alpha_{x}\left(A_{1}\right), A_{2}\right]\right\|\left\|L_{2}\right\| q_{\Delta}\left(L_{1}\right)+\left\|A_{2}\right\|\left\|\left[\alpha_{x}\left(A_{1}\right), L_{2}\right]\right\| q_{\Delta}\left(L_{1}\right),
\end{array}
\end{aligned}
$$

and rapid decay is an immediate consequence of almost locality for all terms but the second one on the right-hand side of this inequality. Using suitable decompositions of $L_{1}$ and $L_{2}$ in terms of elements of $\mathfrak{L}_{0}$ complying pairwise with the special properties exploited in the previous paragraph, the remaining problem of decrease of the mapping $\boldsymbol{x} \mapsto q_{\Delta}\left(\left[\alpha_{x}\left(L_{1}\right), L_{2}\right]\right)$ reduces to the case that has already been solved above, thus completing the proof. 


\section{Chapter 3}

\section{Particle Weights as Asymptotic Plane Waves}

Having analysed in great detail the nets of seminorms $q_{\Delta}$ and $p_{\Delta}$, indexed by the bounded Borel sets $\Delta \subseteq \mathbb{R}^{s+1}$, on $\mathfrak{L}$ and $\mathfrak{C}$, respectively, we now turn to the investigation of the topological dual spaces:

Definition 3.1. (a) The linear functionals on $\mathfrak{C}$ which are continuous with respect to the seminorm $p_{\Delta}$ constitute a vector space $\mathfrak{C}_{\Delta}{ }^{*}$, which is a normed space via

$$
\|\varsigma\|_{\Delta} \doteq \sup \left\{|\varsigma(C)|: C \in \mathfrak{C}, p_{\Delta}(C) \leqslant 1\right\}, \quad \varsigma \in \mathfrak{C}_{\Delta}^{*} .
$$

(b) The topological duals of the locally convex spaces $\left(\mathfrak{L}_{0}, \mathfrak{T}_{q}\right),\left(\mathfrak{L}, \mathfrak{T}_{q}\right)$ and $\left(\mathfrak{C}, \mathfrak{T}_{p}\right)$ are denoted $\mathfrak{L}_{0}{ }^{*}, \mathfrak{L}^{*}$ and $\mathfrak{C}^{*}$, respectively.

Remark. Due to the net property (Proposition 2.9) of the family of seminorms $p_{\Delta}$, a linear functional belongs to the topological dual $\mathfrak{C}^{*}$ of $\left(\mathfrak{C}, \mathfrak{T}_{p}\right)$ if and only if it is continuous with respect to one specific seminorm $p_{\Delta^{\prime}}, \Delta^{\prime}$ a bounded Borel subset of $\mathbb{R}^{s+1}$ [42, Proposition 1.2.8]. Hence

$$
\mathfrak{C}^{*}=\bigcup\left\{\mathfrak{C}_{\Delta}^{*}: \Delta \subseteq \mathbb{R}^{s+1} \text { a bounded Borel set }\right\} .
$$

By continuous linear extension [44, Chapter One, $\S 5,4 .(4)]$, the functionals from $\mathfrak{C}^{*}$ are moreover in one-to-one correspondence with the elements of the topological dual $\overline{\mathfrak{C}}^{*}$ of the complete locally convex space $\left(\overline{\mathfrak{C}}, \overline{\mathfrak{T}}_{p}\right)$. By the same argument, they are furthermore embedded in the topological dual $\mathfrak{A}_{\mathfrak{C}}{ }^{*}$ of $\left(\mathfrak{A}_{\mathfrak{C}}, \overline{\mathfrak{T}}_{p}^{u}\right)$. We shall make use of these properties without special mention.

\subsection{General Properties}

Before proceeding to extract certain elements from $\mathfrak{C}^{*}$ to be interpreted, on the grounds of their specific properties, as representing asymptotic mixtures of particle-like quantities, we are first going to collect a number of important properties common to all functionals from the topological dual of $\mathfrak{C}$ whose proof does not depend on special assumptions. First of all, continuity as established in Proposition 2.16 directly carries over to functionals in $\mathfrak{C}^{*}$. 
Lemma 3.2. Continuous linear functionals $\varsigma \in \mathfrak{C}^{*}$ have the following properties.

(i) The mapping $\mathrm{P}_{+}^{\uparrow} \ni(\Lambda, x) \mapsto \varsigma\left(L_{1}{ }^{*} \alpha_{(\Lambda, x)}\left(L_{2}\right)\right)$ is continuous for arbitrary but fixed $L_{1}, L_{2} \in \mathfrak{L}$.

(ii) The mapping $\mathrm{P}_{+}^{\uparrow} \ni(\Lambda, x) \mapsto \varsigma\left(\alpha_{(\Lambda, x)}(C)\right)$ is continuous for given $C \in \mathfrak{C}$.

Proof. Due to the assumed continuity of $\varsigma$, the assertions follow from Proposition 2.16 in connection with Corollary 2.13.

Every positive functional $\varsigma$ on the ${ }^{*}$-algebra $\mathfrak{C}=\mathfrak{L}^{*} \mathfrak{L}$ defines a non-negative sesquilinear form on $\mathfrak{L}$ through

$$
\langle\cdot \mid \cdot\rangle_{\varsigma}: \mathfrak{L} \times \mathfrak{L} \rightarrow \mathbb{C} \quad\left(L_{1}, L_{2}\right) \mapsto\left\langle L_{1} \mid L_{2}\right\rangle_{\varsigma} \doteq \varsigma\left(L_{1}{ }^{*} L_{2}\right),
$$

and thus induces a seminorm $q_{\varsigma}$ on $\mathfrak{L}$ via

$$
q_{\varsigma}: \mathfrak{L} \rightarrow \mathbb{R}_{+} \quad L \mapsto q_{\varsigma}(L) \doteq\langle L \mid L\rangle_{\varsigma}^{1 / 2}
$$

Denoting by $\mathfrak{N}_{\zeta}$ the null space of $q_{\zeta}$, one can construct the quotient $\mathfrak{L}_{\zeta} \doteq \mathfrak{L} / \mathfrak{N}_{\zeta}$, which is a normed space through the definition

$$
\|\cdot\|_{\varsigma}: \mathfrak{L} / \mathfrak{N}_{\zeta} \rightarrow \mathbb{R}_{+} \quad[L]_{\varsigma} \mapsto\left\|[L]_{\varsigma}\right\|_{\varsigma} \doteq q_{\varsigma}(L),
$$

where we used square brackets to designate the cosets in $\mathfrak{L} / \mathfrak{N}_{\zeta}$. These concepts can be applied to formulate, parallel to Proposition 2.16, differentiability of the Poincaré automorphisms with respect to continuous positive functionals on $\mathfrak{C}$.

Lemma 3.3. Let $\varsigma$ be a continuous positive functional on the ${ }^{*}$-algebra $\mathfrak{C}$, i. e. $\varsigma \in \mathfrak{C}_{+}^{*}$. Then the restriction of the canonical homomorphism

$$
Q_{\varsigma}: \mathfrak{L} \rightarrow \mathfrak{L} / \mathfrak{N}_{\varsigma} \quad L \mapsto Q_{\varsigma}(L) \doteq[L]_{\varsigma}
$$

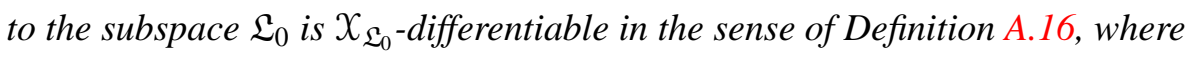

$$
X_{\mathfrak{L}_{0}}=\left\{\Xi_{L_{0}}: L_{0} \in \mathfrak{L}_{0}\right\}
$$

is the family of infinitely often differentiable mappings defined in Proposition 2.16.

Proof. Due to the assumed continuity of the functional $\varsigma$, there exists a bounded Borel set $\Delta$ such that, according to (3.2) in connection with Definition 3.1 and Lemma 2.14, for any $L \in \mathfrak{L}$ there holds the inequality

$$
\left\|[L]_{\varsigma}\right\|_{\zeta}^{2}=q_{\varsigma}(L)^{2}=\varsigma\left(L^{*} L\right) \leqslant\|\varsigma\|_{\Delta} p_{\Delta}\left(L^{*} L\right)=\|\varsigma\|_{\Delta} q_{\Delta}(L)^{2} .
$$

Therefore the linear operator

$$
\mathcal{Q}_{\zeta}\left\lceil\mathfrak{L}_{0}:\left(\mathfrak{L}_{0}, \mathfrak{T}_{q}\right) \rightarrow\left(\mathfrak{L} / \mathfrak{N}_{\zeta},\|\cdot\|_{\zeta}\right)\right.
$$

turns out to be continuous, so that the assertion follows by an application of Corollary A.15 from the result of Proposition 2.16, stating that the mappings

$$
\Xi_{L_{0}}: P_{+}^{\uparrow} \rightarrow\left(\mathfrak{L}_{0}, \mathfrak{T}_{q}\right) \quad(\Lambda, x) \mapsto \Xi_{L_{0}}(\Lambda, x) \doteq \alpha_{(\Lambda, x)}\left(L_{0}\right)
$$

are differentiable for any $L_{0} \in \mathfrak{L}_{0}$ (cf. the remark of that place). 
The next lemmas are concerned with integrability properties of functionals $\varsigma \in \mathfrak{C}^{*}$, parallel to those established in Subsection 2.3.3. The first one, Lemma 3.4, is an immediate consequence of Lemmas 2.17 and 2.18, whereas the second one, Lemma 3.5, prepares the proof of a kind of Cluster Property for positive functionals in $\mathfrak{C}^{*}$, formulated in the subsequent Proposition 3.6.

Lemma 3.4. Let $\varsigma \in \mathfrak{C}^{*}, L_{1}, L_{2} \in \mathfrak{L}$ and $C \in \mathfrak{C}$.

(i) Let $F \in L^{1}\left(\mathrm{P}_{+}^{\uparrow}, d \mu(\Lambda, x)\right)$ have compact support $\mathrm{S}$, then

$$
\begin{aligned}
\varsigma\left(L_{1}{ }^{*} \alpha_{F}\left(L_{2}\right)\right) & =\int d \mu(\Lambda, x) F(\Lambda, x) \varsigma\left(L_{1}^{*} \alpha_{(\Lambda, x)}\left(L_{2}\right)\right), \\
\varsigma\left(\alpha_{F}(C)\right) & =\int d \mu(\Lambda, x) F(\Lambda, x) \varsigma\left(\alpha_{(\Lambda, x)}(C)\right),
\end{aligned}
$$

and there hold the estimates

$$
\begin{gathered}
\left|\varsigma\left(L_{1}{ }^{*} \alpha_{F}\left(L_{2}\right)\right)\right| \leqslant\|F\|_{1}\|\varsigma\|_{\Delta} q_{\Delta}\left(L_{1}\right) \sup _{(\Lambda, x) \in \mathrm{S}} q_{\Delta}\left(\alpha_{(\Lambda, x)}\left(L_{2}\right)\right), \\
\left|\varsigma\left(\alpha_{F}(C)\right)\right| \leqslant\|F\|_{1}\|\varsigma\|_{\Delta} \sup _{(\Lambda, x) \in \mathrm{S}} p_{\Delta}\left(\alpha_{(\Lambda, x)}(C)\right)
\end{gathered}
$$

for any $\Delta$ such that $\varsigma \in \mathfrak{C}_{\Delta}{ }^{*}$.

(ii) For any function $g \in L^{1}\left(\mathbb{R}^{s+1}, d^{s+1} x\right)$

$$
\varsigma\left(L_{1}^{*} \alpha_{g}\left(L_{2}\right)\right)=\int_{\mathbb{R}^{s+1}} d^{s+1} x g(x) \varsigma\left(L_{1}^{*} \alpha_{x}\left(L_{2}\right)\right),
$$

and a bound is given by

$$
\left|\varsigma\left(L_{1}^{*} \alpha_{g}\left(L_{2}\right)\right)\right| \leqslant\|g\|_{1}\|\varsigma\|_{\Delta} q_{\Delta}\left(L_{1}\right) q_{\Delta}\left(L_{2}\right)
$$

for any $\Delta$ satisfying $\varsigma \in \mathfrak{C}_{\Delta}^{*}$.

Proof. Lemmas 2.17 and 2.18 state that

$$
\begin{aligned}
\alpha_{F}\left(L_{2}\right) & =\int d \mu(\Lambda, x) F(\Lambda, x) \alpha_{(\Lambda, x)}\left(L_{2}\right), \\
\alpha_{F}(C) & =\int d \mu(\Lambda, x) F(\Lambda, x) \alpha_{(\Lambda, x)}(C), \\
\alpha_{g}\left(L_{2}\right) & =\int_{\mathbb{R}^{s+1}} d^{s+1} x g(x) \alpha_{x}\left(L_{2}\right)
\end{aligned}
$$

exist in the complete locally convex spaces $\left(\mathfrak{A}_{\mathfrak{L}}, \overline{\mathfrak{T}}_{q}^{u}\right)$ and $\left(\mathfrak{A}_{\mathfrak{C}}, \overline{\mathfrak{T}}_{p}^{u}\right)$, respectively. Now, the functional $\zeta$, which lies in $\mathfrak{A}_{\mathfrak{C}}^{*}$ according to the remark following Definition 3.1, is linear and continuous with respect to $\alpha_{F}(C) \in \mathfrak{A}_{\mathfrak{C}}$ and, by Corollary 2.13, also with respect to both $\alpha_{F}\left(L_{2}\right), \alpha_{g}\left(L_{2}\right) \in \mathfrak{A}_{\mathfrak{L}}$. Therefore it commutes with the locally convex integrals [26, Proposition II.5.7 adapted to integrals in locally convex spaces], which proves the assertion. The annexed estimates are a further simple application of the results contained in Lemmas 2.17 and 2.18. 
Lemma 3.5. Let $L^{\prime} \in \mathfrak{L}$ and let $L \in \mathfrak{L}(\Gamma)=\mathfrak{L} \cap \widetilde{\mathfrak{A}}(\Gamma), \Gamma \subseteq \mathbb{R}^{s+1}$ compact, i. e. L has energy-momentum transfer in $\Gamma$. If $\varsigma \in \mathfrak{C}^{*+}$ is a positive functional which belongs to $\mathfrak{C}_{\Delta}^{*}$ and $\Delta^{\prime}$ denotes any bounded Borel set containing $\Delta+\Gamma$, then

$$
\int_{\mathbb{R}^{s}} d^{s} x \varsigma\left(L^{*} \alpha_{x}\left(L^{\prime *} L^{\prime}\right) L\right) \leqslant\|\varsigma\|_{\Delta} q_{\Delta}(L)^{2} q_{\Delta^{\prime}}\left(L^{\prime}\right)^{2} .
$$

Proof. Let $\boldsymbol{K}$ be an arbitrary compact subset of $\mathbb{R}^{s}$ and note that

$$
\int_{\boldsymbol{K}} d^{s} x \alpha_{x}\left(L^{* *} L^{\prime}\right) \in \mathfrak{A}
$$

Thus, according to the construction of $\mathfrak{C}$,

$$
\int_{\boldsymbol{K}} d^{s} x L^{*} \alpha_{x}\left(L^{\prime *} L^{\prime}\right) L=L^{*} \int_{\boldsymbol{K}} d^{s} x \alpha_{x}\left(L^{\prime *} L^{\prime}\right) L
$$

belongs to the algebra of counters and exists furthermore as an integral in the locally convex space $\left(\mathfrak{A}_{\mathfrak{C}}, \overline{\mathfrak{T}}_{p}^{u}\right)$. Therefore the functional $\varsigma \in \mathfrak{A}_{\mathfrak{C}}{ }^{*}$ can be interchanged with the integral [26, Proposition II.5.7] to give

$$
\int_{\boldsymbol{K}} d^{s} x \varsigma\left(L^{*} \alpha_{x}\left(L^{\prime *} L^{\prime}\right) L\right)=\varsigma\left(L^{*} \int_{\boldsymbol{K}} d^{s} x \alpha_{x}\left(L^{\prime *} L^{\prime}\right) L\right)
$$

Application of Lemma 2.12 then leads to the estimate

$$
\begin{aligned}
0 \leqslant \int_{\boldsymbol{K}} d^{s} x \varsigma\left(L^{*} \alpha_{x}\left(L^{\prime *} L^{\prime}\right) L\right) & \leqslant\|\varsigma\|_{\Delta} p_{\Delta}\left(L^{*} \int_{\boldsymbol{K}} d^{s} x \alpha_{x}\left(L^{\prime *} L^{\prime}\right) L\right) \\
& \leqslant\|\varsigma\|_{\Delta} q_{\Delta}(L)^{2}\left\|E\left(\Delta^{\prime}\right) \int_{\boldsymbol{K}} d^{s} x \alpha_{x}\left(L^{\prime *} L^{\prime}\right) E\left(\Delta^{\prime}\right)\right\|=\|\varsigma\|_{\Delta} q_{\Delta}(L)^{2}\left\|Q_{\Delta^{\prime}, \boldsymbol{K}}^{\left(L^{\prime *} L^{\prime}\right)}\right\|,
\end{aligned}
$$

where we made use of the positivity of $\varsigma$. The above inequality survives in the limit $\boldsymbol{K} \nearrow \mathbb{R}^{s}$ and the convergence of the right-hand side to a finite real number establishes the integrability of the function

$$
\mathbb{R}^{s} \ni \boldsymbol{x} \mapsto \varsigma\left(L^{*} \alpha_{x}\left(L^{\prime *} L^{\prime}\right) L\right)
$$

as a consequence of the Monotone Convergence Theorem [26, II.2.7]. In view of (2.17a), one finally arrives at the asserted bound

$$
\int_{\mathbb{R}^{s}} d^{s} x \varsigma\left(L^{*} \alpha_{x}\left(L^{\prime *} L^{\prime}\right) L\right) \leqslant\|\varsigma\|_{\Delta} q_{\Delta}(L)^{2}\left\|Q_{\Delta^{\prime}}^{\left(L^{\prime *} L^{\prime}\right)}\right\|=\|\varsigma\|_{\Delta} q_{\Delta}(L)^{2} q_{\Delta^{\prime}}\left(L^{\prime}\right)^{2} .
$$

After these preparations we are in a position to prove the announced Cluster Property for positive functionals in $\mathfrak{C}^{*}$.

Proposition 3.6 (Cluster Property). Let $L_{i}$ and $L_{i}^{\prime}$ be elements of $\mathfrak{L}_{0}$ and let $A_{i} \in \mathfrak{A}$, $i=1,2$, be almost local operators, then the function

$$
\mathbb{R}^{s} \ni \boldsymbol{x} \mapsto \varsigma\left(\left(L_{1}{ }^{*} A_{1} L_{1}^{\prime}\right) \alpha_{\boldsymbol{x}}\left(L_{2}{ }^{*} A_{2} L_{2}^{\prime}\right)\right) \in \mathbb{C}
$$

is an element of $L^{1}\left(\mathbb{R}^{s}, d^{s} x\right)$ for any $\varsigma \in \mathfrak{C}^{*+}$ and satisfies

$$
\int_{\mathbb{R}^{s}} d^{s} x\left|\varsigma\left(\left(L_{1}{ }^{*} A_{1} L_{1}^{\prime}\right) \alpha_{x}\left(L_{2}{ }^{*} A_{2} L_{2}^{\prime}\right)\right)\right| \leqslant\|\varsigma\|_{\Delta} M_{\Delta}
$$

for any bounded Borel set $\Delta$ for which $\varsigma$ belongs to $\mathfrak{C}_{\Delta}{ }^{*}$, where the constant $M_{\Delta}$ depends on $\Delta$ and the operators involved. 
Proof. First, we re-write the argument $\left(L_{1}{ }^{*} A_{1} L_{1}^{\prime}\right) \alpha_{x}\left(L_{2}{ }^{*} A_{2} L_{2}^{\prime}\right)$, commuting the operators $A_{1} L_{1}^{\prime}$ and $\alpha_{x}\left(L_{2}{ }^{*} A_{2}\right)$, to get

$$
\begin{aligned}
& \left(L_{1}{ }^{*} A_{1} L_{1}^{\prime}\right) \alpha_{x}\left(L_{2}{ }^{*} A_{2} L_{2}^{\prime}\right) \\
& \quad=L_{1}{ }^{*}\left[A_{1} L_{1}^{\prime}, \alpha_{x}\left(L_{2}{ }^{*} A_{2}\right)\right] \alpha_{x}\left(L_{2}^{\prime}\right)+L_{1}{ }^{*} \alpha_{x}\left(L_{2}{ }^{*} A_{2}\right) A_{1} L_{1}^{\prime} \alpha_{x}\left(L_{2}^{\prime}\right) .
\end{aligned}
$$

This implies

$$
\begin{aligned}
& \left|\varsigma\left(\left(L_{1}{ }^{*} A_{1} L_{1}^{\prime}\right) \alpha_{x}\left(L_{2}{ }^{*} A_{2} L_{2}^{\prime}\right)\right)\right| \\
& \quad \leqslant\left|\varsigma\left(L_{1}{ }^{*}\left[A_{1} L_{1}^{\prime}, \alpha_{x}\left(L_{2}{ }^{*} A_{2}\right)\right] \alpha_{x}\left(L_{2}^{\prime}\right)\right)\right|+\left|\zeta\left(L_{1}{ }^{*} \alpha_{x}\left(L_{2}{ }^{*} A_{2}\right) A_{1} L_{1}^{\prime} \alpha_{x}\left(L_{2}^{\prime}\right)\right)\right|,
\end{aligned}
$$

where the first term on the right-hand side is evidently integrable over $\mathbb{R}^{s}$, due to almost locality of the operators encompassed by the commutator. For $\varsigma \in \mathfrak{C}_{\Delta}{ }^{*}$ we have the estimate

$$
\begin{aligned}
\int_{\mathbb{R}^{s}} d^{s} x\left|\varsigma\left(L_{1}{ }^{*}\left[A_{1} L_{1}^{\prime}, \alpha_{x}\left(L_{2}{ }^{*} A_{2}\right)\right] \alpha_{x}\left(L_{2}^{\prime}\right)\right)\right| & \\
& \leqslant\|\varsigma\|_{\Delta} q_{\Delta}\left(L_{1}\right) q_{\Delta}\left(L_{2}^{\prime}\right) \int_{\mathbb{R}^{s}} d^{s} x\left\|\left[A_{1} L_{1}^{\prime}, \alpha_{x}\left(L_{2}{ }^{*} A_{2}\right)\right]\right\| .
\end{aligned}
$$

The second term can be estimated by use of the Cauchy-Schwarz inequality applied to the positive functional $\varsigma$ :

$$
\begin{aligned}
& 2\left|\varsigma\left(L_{1}{ }^{*} \alpha_{x}\left(L_{2}{ }^{*} A_{2}\right) A_{1} L_{1}^{\prime} \alpha_{x}\left(L_{2}^{\prime}\right)\right)\right| \\
& \leqslant 2 \varsigma\left(L_{1}{ }^{*} \alpha_{x}\left(L_{2}{ }^{*} A_{2} A_{2}{ }^{*} L_{2}\right) L_{1}\right)^{1 / 2} \varsigma\left(\alpha_{x}\left(L_{2}^{\prime *}\right) L_{1}^{\prime *} A_{1}{ }^{*} A_{1} L_{1}^{\prime} \alpha_{x}\left(L_{2}^{\prime}\right)\right)^{1 / 2} \\
& \quad=\inf _{\lambda>0}\left(\lambda^{-1} \varsigma\left(L_{1}{ }^{*} \alpha_{x}\left(L_{2}{ }^{*} A_{2} A_{2}{ }^{*} L_{2}\right) L_{1}\right)+\lambda \varsigma\left(\alpha_{x}\left(L_{2}^{\prime *}\right) L_{1}^{\prime *} A_{1}{ }^{*} A_{1} L_{1}^{\prime} \alpha_{x}\left(L_{2}^{\prime}\right)\right)\right) .
\end{aligned}
$$

Integration of the first term on the right-hand side is possible according to the previous Lemma 3.5 and gives

$$
\int_{\mathbb{R}^{s}} d^{s} x \varsigma\left(L_{1}{ }^{*} \alpha_{x}\left(L_{2}{ }^{*} A_{2} A_{2}{ }^{*} L_{2}\right) L_{1}\right) \leqslant\|\varsigma\|_{\Delta} q_{\Delta}\left(L_{1}\right)^{2} q_{\Delta_{1}}\left(A_{2}{ }^{*} L_{2}\right)^{2},
$$

where $\Delta_{1}$ is any bounded Borel set containing the sum of $\Delta$ and the energy-momentum transfer $\Gamma_{1}$ of $L_{1}$. Concerning the second term on the right of (3.12), we get, upon commuting $\alpha_{x}\left(L_{2}^{\prime *}\right)$ and $\alpha_{x}\left(L_{2}^{\prime}\right)$ to the interior,

$$
\begin{aligned}
& \zeta\left(\alpha_{x}\left(L_{2}^{\prime *}\right) L_{1}^{\prime *} A_{1}{ }^{*} A_{1} L_{1}^{\prime} \alpha_{x}\left(L_{2}^{\prime}\right)\right) \\
& \leqslant\left|\varsigma\left(\left[\alpha_{x}\left(L_{2}^{\prime *}\right), L_{1}^{\prime *}\right] A_{1}{ }^{*} A_{1} L_{1}^{\prime} \alpha_{x}\left(L_{2}^{\prime}\right)\right)\right|+\mid \\
& \begin{aligned}
& \left(L_{1}^{\prime *} \alpha_{x}\left(L_{2}^{\prime *}\right) A_{1}{ }^{*} A_{1}\left[L_{1}^{\prime}, \alpha_{x}\left(L_{2}^{\prime}\right)\right]\right) \mid \\
& +\left\|A_{1}\right\|^{2}\left|\varsigma\left(L_{1}^{\prime *} \alpha_{x}\left(L_{2}^{\prime *} L_{2}^{\prime}\right) L_{1}^{\prime}\right)\right|,
\end{aligned}
\end{aligned}
$$

where again use was made of the positivity of $\varsigma$. The rapid decay of commutators of almost local operators with respect to the $q_{\Delta}$-seminorm established in Lemma 2.19 of Subsection 2.3.4 can be combined with Lemma 3.5 to show integrability over $\mathbb{R}^{s}$ :

$$
\begin{aligned}
& \int_{\mathbb{R}^{s}} d^{s} x \varsigma\left(\alpha_{x}\left(L_{2}^{\prime *}\right) L_{1}^{\prime *} A_{1}{ }^{*} A_{1} L_{1}^{\prime} \alpha_{x}\left(L_{2}^{\prime}\right)\right) \\
& \leqslant\|\varsigma\|_{\Delta}\left\|A_{1}\right\|^{2}\left(q_{\Delta}\left(L_{1}^{\prime}\right)^{2} q_{\Delta_{1}^{\prime}}\left(L_{2}^{\prime}\right)^{2}+\left(\left\|L_{1}^{\prime}\right\| q_{\Delta}\left(L_{2}^{\prime}\right)+\left\|L_{2}^{\prime}\right\| q_{\Delta}\left(L_{1}^{\prime}\right)\right)\right. \\
&\left.\cdot \int_{\mathbb{R}^{s}} d^{s} x q_{\Delta}\left(\left[L_{1}^{\prime}, \alpha_{x}\left(L_{2}^{\prime}\right)\right]\right)\right)
\end{aligned}
$$


which holds for any bounded Borel set $\Delta_{1}^{\prime} \supseteq \Delta+\Gamma_{1}^{\prime}$, where $\Gamma_{1}^{\prime}$ denotes the energymomentum transfer of $L_{1}^{\prime}$. By (3.14) and (3.15), the left-hand side of (3.12) turns out to be integrable, and a bound for this integral is proportional to $\|\varsigma\|_{\Delta}$. In connection with (3.11) this establishes the assertion for a suitable constant $M_{\Delta}$ that can be deduced from relations (3.11), (3.12), (3.14) and (3.15).

The Cluster Property has been proved above under the fairly general assumption of almost locality of the operators involved. If for given $L_{1}, L_{2} \in \mathfrak{L}$ the mapping

$$
\mathbb{R}^{s} \ni \boldsymbol{x} \mapsto p_{\Delta}\left(L_{1}{ }^{*} \alpha_{\boldsymbol{x}}\left(L_{2}\right)\right)
$$

happens to belong to the space $L^{1}\left(\mathbb{R}^{s}, d^{s} x\right)$ for the bounded Borel set $\Delta$, (3.8) is obviously fulfilled in case that $\varsigma \in \mathfrak{C}^{*}$ belongs to $\mathfrak{C}_{\Delta}^{*}$. As an example consider almost local operators $L_{1}^{\prime}, L_{2}^{\prime} \in \mathfrak{L}$ having energy-momentum transfer $\Gamma_{1}$ and $\Gamma_{2}$, respectively, such that $\left(\Delta+\Gamma_{1}+\Gamma_{2}\right) \cap \bar{V}_{+}=\emptyset$. This implies $L_{1}^{\prime} \alpha_{x}\left(L_{2}^{\prime}\right) E(\Delta)=0$ for any $\boldsymbol{x} \in \mathbb{R}^{s}$ and, by Lemmas 2.11 and 2.12, $p_{\Delta}\left(L_{1}^{\prime *} \alpha_{x}\left(L_{2}^{\prime *}\right) L_{1}^{\prime} \alpha_{x}\left(L_{2}^{\prime}\right)\right)=0$. An application of Lemma 2.12 in connection with translation invariance of $q_{\Delta}$ (Lemma 2.15) then yields for the counters $C_{i}^{\prime} \doteq L_{i}^{\prime *} L_{i}^{\prime}, i=1,2$,

$$
\begin{aligned}
p_{\Delta}\left(C_{1}^{\prime *} \alpha_{x}\left(C_{2}^{\prime}\right)\right)= & p_{\Delta}\left(L_{1}^{\prime *} L_{1}^{\prime} \alpha_{x}\left(L_{2}^{\prime *} L_{2}^{\prime}\right)\right) \\
& =p_{\Delta}\left(L_{1}^{\prime *}\left[L_{1}^{\prime}, \alpha_{x}\left(L_{2}^{\prime *}\right)\right] \alpha_{x}\left(L_{2}^{\prime}\right)\right) \leqslant\left\|\left[L_{1}^{\prime}, \alpha_{x}\left(L_{2}^{\prime *}\right)\right]\right\| q_{\Delta}\left(L_{1}^{\prime}\right) q_{\Delta}\left(L_{2}^{\prime}\right),
\end{aligned}
$$

where, due to the assumed almost locality of $L_{1}^{\prime}$ and $L_{2}^{\prime}$, the right-hand side is seen to belong to $L^{1}\left(\mathbb{R}^{s}, d^{s} x\right)$. The integrability of a mapping $\boldsymbol{x} \mapsto p_{\Delta}\left(L_{1}{ }^{*} \alpha_{\boldsymbol{x}}\left(L_{2}\right)\right), L_{1}, L_{2} \in \mathfrak{L}$, has another consequence concerning weakly convergent nets $\left\{\zeta_{\imath}: \imath \in J\right\}$ of functionals from $\mathfrak{C}^{*}$, which are contained in bounded subsets of $\mathfrak{C}_{\Delta}{ }^{*}$ with respect to the norm $\|\cdot\|_{\Delta}$ : a kind of Dominated Convergence Theorem.

Lemma 3.7. Let $L_{1}, L_{2} \in \mathfrak{L}$ be such that $\boldsymbol{x} \mapsto p_{\Delta}\left(L_{1}{ }^{*} \alpha_{x}\left(L_{2}\right)\right)$ is integrable and consider the weakly convergent net $\left\{\varsigma_{\mathfrak{l}}: \mathfrak{\imath} \in J\right\}$ in the D-ball of $\mathfrak{C}_{\Delta}{ }^{*}$ with limit $\varsigma$.This means that for any $C \in \mathfrak{C}$

$$
\lim _{l} \varsigma_{l}(C)=\varsigma(C)
$$

and for any $\mathrm{l} \in J$

$$
\begin{aligned}
\left|\varsigma_{\mathrm{I}}(C)\right| & \leqslant D \cdot p_{\Delta}(C), \\
|\varsigma(C)| & \leqslant D \cdot p_{\Delta}(C),
\end{aligned}
$$

the latter relation being implied by the former. Then

$$
\int_{\mathbb{R}^{s}} d^{s} x \varsigma\left(L_{1}^{*} \alpha_{x}\left(L_{2}\right)\right)=\lim _{1} \int_{\mathbb{R}^{s}} d^{s} x \varsigma_{1}\left(L_{1}^{*} \alpha_{x}\left(L_{2}\right)\right) .
$$

Proof. As implied by Proposition 2.16 and Corollary 2.13, $\boldsymbol{x} \mapsto L_{1}{ }^{*} \alpha_{x}\left(L_{2}\right)$ is a continuous mapping on $\mathbb{R}^{s}$ with respect to the $p_{\Delta}$-topology, hence it is uniformly continuous on any compact set $\boldsymbol{K}$. This means that to $\varepsilon>0$ there exists $\delta>0$ such that $\boldsymbol{x}, \boldsymbol{x}^{\prime} \in \boldsymbol{K}$ and $\left|\boldsymbol{x}-\boldsymbol{x}^{\prime}\right|<\delta$ imply

$$
p_{\Delta}\left(L_{1}{ }^{*} \alpha_{x}\left(L_{2}\right)-L_{1}^{*} \alpha_{x^{\prime}}\left(L_{2}\right)\right)<\frac{\varepsilon}{6 D|\boldsymbol{K}|},
$$


where $|\boldsymbol{K}|$ denotes the $s$-dimensional volume of $\boldsymbol{K}$. Consequently, under the above assumption on $\boldsymbol{x}$ and $\boldsymbol{x}^{\prime}$, we infer from (3.16)

$$
\begin{aligned}
\left|\varsigma_{\imath}\left(L_{1}{ }^{*} \alpha_{x}\left(L_{2}\right)\right)-\varsigma_{\imath}\left(L_{1}{ }^{*} \alpha_{x^{\prime}}\left(L_{2}\right)\right)\right| & =\left|\varsigma_{\imath}\left(L_{1}{ }^{*} \alpha_{x}\left(L_{2}\right)-L_{1}{ }^{*} \alpha_{x^{\prime}}\left(L_{2}\right)\right)\right|<\frac{\varepsilon}{6|\boldsymbol{K}|}, \\
\left|\varsigma\left(L_{1}{ }^{*} \alpha_{x}\left(L_{2}\right)\right)-\varsigma\left(L_{1}{ }^{*} \alpha_{x^{\prime}}\left(L_{2}\right)\right)\right| & =\left|\varsigma\left(L_{1}{ }^{*} \alpha_{x}\left(L_{2}\right)-L_{1}{ }^{*} \alpha_{x^{\prime}}\left(L_{2}\right)\right)\right|<\frac{\varepsilon}{6|\boldsymbol{K}|} .
\end{aligned}
$$

By compactness of $\boldsymbol{K}$, there exist finitely many elements $\boldsymbol{x}_{1}, \ldots, \boldsymbol{x}_{N} \in \boldsymbol{K}$ such that the $\delta$-balls around these points cover all of $\boldsymbol{K}$; moreover, since $\varsigma$ is the weak limit of the net $\left\{\varsigma_{\imath}: \imath \in J\right\}$, we can find $\iota_{0} \in J$ such that $\imath \succ \mathfrak{\imath}_{0}$ implies

$$
\left|\varsigma\left(L_{1}^{*} \alpha_{x_{i}}\left(L_{2}\right)\right)-\varsigma_{\mathfrak{l}}\left(L_{1}^{*} \alpha_{x_{i}}\left(L_{2}\right)\right)\right|<\frac{\varepsilon}{6|\boldsymbol{K}|}
$$

for any $i=1, \ldots, N$. Now, for $\mathrm{\imath} \in J$ and $k \in\{1, \ldots, N\}$,

$$
\begin{aligned}
& \left|\varsigma\left(L_{1}{ }^{*} \alpha_{x}\left(L_{2}\right)\right)-\varsigma_{\imath}\left(L_{1}{ }^{*} \alpha_{x}\left(L_{2}\right)\right)\right| \\
& \leqslant\left|\varsigma\left(L_{1}{ }^{*} \alpha_{x}\left(L_{2}\right)\right)-\varsigma\left(L_{1}{ }^{*} \alpha_{x_{i}}\left(L_{2}\right)\right)\right|+\left|\varsigma\left(L_{1}{ }^{*} \alpha_{x_{i}}\left(L_{2}\right)\right)-\varsigma_{\imath}\left(L_{1}{ }^{*} \alpha_{x_{i}}\left(L_{2}\right)\right)\right| \\
& +\left|\varsigma_{l}\left(L_{1}{ }^{*} \alpha_{x_{i}}\left(L_{2}\right)\right)-\varsigma_{l}\left(L_{1}{ }^{*} \alpha_{x}\left(L_{2}\right)\right)\right|,
\end{aligned}
$$

and, selecting for $\boldsymbol{x} \in \boldsymbol{K}$ an appropriate $\boldsymbol{x}_{k}$ in a distance less than $\delta$, we can put the above results together to get the estimate

$$
\left|\varsigma\left(L_{1}^{*} \alpha_{x}\left(L_{2}\right)\right)-\varsigma_{1}\left(L_{1}^{*} \alpha_{x}\left(L_{2}\right)\right)\right|<\frac{\varepsilon}{2|\boldsymbol{K}|},
$$

which holds for any $\boldsymbol{x} \in \boldsymbol{K}$ and $\boldsymbol{\imath} \succ \mathfrak{1}_{0}$. Thus weak (i. e. pointwise) convergence of the net $\left\{\varsigma_{l}: \imath \in J\right\}$ is indeed uniform convergence on compact subsets of $\mathbb{R}^{s}$. Upon integration over $\boldsymbol{K}$ we arrive at

$$
\begin{aligned}
\mid \int_{\boldsymbol{K}} d^{s} x\left(\varsigma\left(L_{1}{ }^{*} \alpha_{x}\left(L_{2}\right)\right)-\varsigma_{l}(\right. & \left.\left.L_{1}{ }^{*} \alpha_{x}\left(L_{2}\right)\right)\right) \mid \\
& \leqslant \int_{\boldsymbol{K}} d^{s} x\left|\varsigma\left(L_{1}{ }^{*} \alpha_{x}\left(L_{2}\right)\right)-\varsigma_{l}\left(L_{1}{ }^{*} \alpha_{x}\left(L_{2}\right)\right)\right|<\frac{\varepsilon}{2} .
\end{aligned}
$$

Now, by assumption

$$
\int_{\mathbb{R}^{s}} d^{s} x p_{\Delta}\left(L_{1}^{*} \alpha_{x}\left(L_{2}\right)\right)<\infty
$$

so that to $\varepsilon>0$ there exists a compact subset $\boldsymbol{K}_{\varepsilon}$ satisfying

$$
\int_{C_{\varepsilon}} d^{s} x p_{\Delta}\left(L_{1}^{*} \alpha_{x}\left(L_{2}\right)\right)<\frac{\varepsilon}{4 D} .
$$

Then, as a consequence of (3.16a) and (3.16b), we get for any $\imath \in J$

$$
\begin{gathered}
\int_{\int_{\boldsymbol{K}_{\varepsilon}} d^{s} x\left|\varsigma_{\imath}\left(L_{1}{ }^{*} \alpha_{x}\left(L_{2}\right)\right)\right|}<\frac{\varepsilon}{4}, \\
\int_{\complement \boldsymbol{K}_{\varepsilon}} d^{s} x\left|\varsigma\left(L_{1}{ }^{*} \alpha_{x}\left(L_{2}\right)\right)\right|<\frac{\varepsilon}{4} .
\end{gathered}
$$


Combining (3.19) with (3.18) for the compact set $\boldsymbol{K}_{\varepsilon}$ yields for $\boldsymbol{\imath} \succ \mathfrak{l}_{0}$ (note, that $\boldsymbol{1}_{0}$ only depends on $\varepsilon$ )

$$
\begin{aligned}
& \left|\int_{\mathbb{R}^{s}} d^{s} x \varsigma\left(L_{1}{ }^{*} \alpha_{x}\left(L_{2}\right)\right)-\int_{\mathbb{R}^{s}} d^{s} x \varsigma_{l}\left(L_{1}{ }^{*} \alpha_{x}\left(L_{2}\right)\right)\right| \\
& \leqslant \int_{\mathrm{CK}_{\varepsilon}} d^{s} x\left|\varsigma\left(L_{1}{ }^{*} \alpha_{x}\left(L_{2}\right)\right)\right|+\int_{\boldsymbol{K}} d^{s} x\left|\varsigma\left(L_{1}{ }^{*} \alpha_{x}\left(L_{2}\right)\right)-\varsigma_{l}\left(L_{1}{ }^{*} \alpha_{x}\left(L_{2}\right)\right)\right| \\
& \quad+\int_{\mathrm{CK}_{\varepsilon}} d^{s} x\left|\varsigma_{l}\left(L_{1}{ }^{*} \alpha_{x}\left(L_{2}\right)\right)\right|<\frac{\varepsilon}{4}+\frac{\varepsilon}{2}+\frac{\varepsilon}{4}=\varepsilon .
\end{aligned}
$$

By arbitrariness of $\varepsilon$ this proves the possibility to interchange integration and the limit with respect to $l$ as asserted in (3.17).

The spectral support of not necessarily positive functionals $\varsigma \in \mathfrak{C}^{*}$ (considered as distributions) depends, as expressed in the subsequent proposition, on the bounded Borel sets $\Delta$ for which $\varsigma \in \mathfrak{C}_{\Delta}{ }^{*}$. This property will prove to be of importance when it comes to defining the energy-momentum of particle weights.

Proposition 3.8 (Spectral Property). Let $L_{1}, L_{2} \in \mathfrak{L}$ and $\varsigma \in \mathfrak{C}^{*}$. Then the support of the Fourier transform of the distribution

$$
\mathbb{R}^{s+1} \ni x \mapsto \varsigma\left(L_{1}{ }^{*} \alpha_{x}\left(L_{2}\right)\right) \in \mathbb{C}
$$

is contained in the shifted light cone $\bar{V}_{+}-q$ for some $q \in \bar{V}_{+}$. More specifically, $q$ is such that a bounded Borel set $\Delta$, satisfying $\varsigma \in \mathfrak{C}_{\Delta}{ }^{*}$, is contained in $q-\bar{V}_{+}$.

Proof. If a function $g$ belongs to the space $L^{1}\left(\mathbb{R}^{s+1}, d^{s+1} x\right)$, then the operator

$$
\alpha_{g}\left(L_{2}\right)=\int_{\mathbb{R}^{s+1}} d^{s+1} x g(x) \alpha_{x}\left(L_{2}\right)
$$

lies in $\mathfrak{A}_{\mathfrak{L}}$, according to Lemma 2.18, and has energy-momentum transfer in supp $\tilde{g}$, the support of the Fourier transform of $g$. If this happens to satisfy supp $\tilde{g} \subseteq \complement\left(\bar{V}_{+}-\Delta\right)$, we infer $\alpha_{g}\left(L_{2}\right) E(\Delta)=0$ and henceforth, by Lemma 2.11, $q_{\Delta}\left(\alpha_{g}\left(L_{2}\right)\right)=0$. Since $\varsigma$ is assumed to belong to $\mathfrak{C}_{\Delta}^{*}$, Lemma 3.4 results in

$$
\left|\int_{\mathbb{R}^{s+1}} d^{s+1} x g(x) \varsigma\left(L_{1}{ }^{*} \alpha_{x}\left(L_{2}\right)\right)\right|=\left|\varsigma\left(L_{1}^{*} \alpha_{g}\left(L_{2}\right)\right)\right| \leqslant\|\varsigma\|_{\Delta} q_{\Delta}\left(L_{1}\right) q_{\Delta}\left(\alpha_{g}\left(L_{2}\right)\right),
$$

which, according to the preceding considerations, entails

$$
\int_{\mathbb{R}^{s+1}} d^{s+1} x g(x) \varsigma\left(L_{1}^{*} \alpha_{x}\left(L_{2}\right)\right)=0 .
$$

Now, let $g^{\prime}$ be an arbitrary function from $L^{1}\left(\mathbb{R}^{s+1}, d^{s+1} x\right)$ with supp $\tilde{g^{\prime}} \subseteq \complement\left(\bar{V}_{+}-q\right)$, $\Delta \subseteq q-\bar{V}_{+}$, then supp $\tilde{g}^{\prime} \subseteq \complement\left(\bar{V}_{+}-\Delta\right)$, so that (3.20) is fulfilled for any function of this kind, proving the assertion. 


\subsection{Asymptotic Functionals}

Now we turn to functionals in $\mathfrak{C}^{*}$ that carry additional properties, reflecting the fact that the present investigation is concerned with the structure of the totality of physical states at asymptotic times (scattering states). The temporal development of such a state of bounded energy, $\omega \in \mathcal{S}(\Delta), \Delta$ a bounded Borel set, can be explored by considering an integral of the following shape:

$$
\int_{\mathbb{R}^{s}} d^{s} v h(v) \omega\left(\alpha_{(\tau, \tau v)}(C)\right)
$$

where $h$ denotes a bounded measurable function on the unit ball of $\mathbb{R}^{s}$, where the elements $v$ represent velocities. Apart from this function, (3.21) coincides with the integral (2.5) encountered on page 8 in the heuristic considerations of Chapter 2. The investigations carried through in that part (cf. Proposition 2.7) imply that (3.21) takes on a finite value for any counter $C \in \mathfrak{C}$ at any time $\tau$ and, according to Lemma 2.10, the integral (3.21) even exists for all $C \in \mathfrak{A}_{\mathfrak{C}}$.

The physical interpretation is as follows: Consider a function $h$ of bounded support $\mathrm{V} \subseteq \mathbb{R}^{s} \backslash\{0\}$ in velocity space, then the integral (3.21) corresponds to summing up, for given time $\tau$, the expectation values of measurements of $C$ in the state $\omega$, where these measurements extend over the bounded section $\tau \cdot V$ of configuration space. For growing $\tau$ the distance of this portion from the origin increases together with its total extension. More exactly, the measurements take place in a cone with apex at the point 0 of space-time, its direction is determined by the support of $h$, and for different times $\tau$ the counter $C$ is set up in specific parts of that cone, their extension growing as $|\tau|^{s}$ (compensating for the quantum mechanical spreading of wave packets) while their distance from the origin increases proportional to $|\tau|$. If the physical state $\omega$ has, in the limit of large (positive or negative) times, evolved into a configuration containing a particle (incoming or outgoing) travelling with velocity $\boldsymbol{v}_{0} \in \mathrm{V}$, then a counter $C_{0}$, sensitive for that specific particle, is expected to asymptotically produce a stable signal under the above experimental conditions.

The mathematical equivalent of this situation is the existence of limits of the above integral at asymptotic times, evaluated for the counter $C_{0}$ and a function $h_{0}$ with support containing $\boldsymbol{v}_{0}$. Thus the problem has to be settled in which (topological) sense such limits can be established, if they happen to exist at all. To tackle this assignment we turn to a slightly modified version of (3.21) in Definition 3.9, involving, for technical reasons, a certain time average.

Definition 3.9. Let $\Delta$ be a bounded Borel subset of $\mathbb{R}^{s+1}$, let $\omega \in \mathcal{S}(\Delta)$ denote a physical state of bounded energy and let $\boldsymbol{v} \mapsto h(\boldsymbol{v})$ be a bounded measurable function on the unit ball of $\mathbb{R}^{s}$. Furthermore suppose that $t \mapsto T(t)$ is a continuous real-valued function, approaching $+\infty$ or $-\infty$ for asymptotic positive or negative times, respectively, not as fast as $|t|$. Then we define a net $\left\{\rho_{h, t}: t \in \mathbb{R}\right\}$ of linear functionals on $\mathfrak{C}$ by setting

$$
\begin{aligned}
\rho_{h, t}(C) & \doteq T(t)^{-1} \int_{t}^{t+T(t)} d \tau \tau^{s} \int_{\mathbb{R}^{s}} d^{s} v h(\boldsymbol{v}) \omega\left(\alpha_{(\tau, \tau v)}(C)\right) \\
& =T(t)^{-1} \int_{t}^{t+T(t)} d \tau \int_{\mathbb{R}^{s}} d^{s} x h\left(\tau^{-1} \boldsymbol{x}\right) \omega\left(\alpha_{(\tau, \boldsymbol{x})}(C)\right), \quad C \in \mathfrak{C} .
\end{aligned}
$$


Under the above assumptions the functionals $\rho_{h, t}$ turn out to be continuous with respect to the seminorm $p_{\Delta}$ pertaining to the energy-momentum support of the physical state $\omega()=.\omega(E(\Delta) . E(\Delta))$, i. e. $\rho_{h, t} \in \mathfrak{C}_{\Delta}{ }^{*}$. This can be seen as follows: First, note that the operators $U(\tau)$ implementing time translations commute with $E(\Delta)$, so that

$$
\omega\left(E(\Delta) \alpha_{(\tau, x)}(C) E(\Delta)\right)=\omega\left(U(\tau) E(\Delta) \alpha_{x}(C) E(\Delta) U(\tau)^{*}\right),
$$

which allows (3.22) to be re-written as

$$
\rho_{h, t}(C)=T(t)^{-1} \int_{t}^{t+T(t)} d \tau \int_{\mathbb{R}^{s}} d^{s} x h\left(\tau^{-1} \boldsymbol{x}\right) \omega\left(U(\tau) E(\Delta) \alpha_{x}(C) E(\Delta) U(\tau)^{*}\right)
$$

Now, all the functionals $\omega\left(U(\tau) . U(\tau)^{*}\right), \tau \in \mathbb{R}$, belong to $\mathfrak{B}(\mathcal{H})_{*, 1}$, so that the absolute value of $\rho_{h, t}(C)$ can be estimated, making use of $p_{\Delta}$ as defined in (2.17b). Abbreviating the interval of $\tau$-integration depending on $t$ as $I_{t}$, this gives

$$
\begin{aligned}
& \left|\rho_{h, t}(C)\right| \leqslant \sup _{\tau \in I_{t}}\left|\int_{\mathbb{R}^{s}} d^{s} x h\left(\tau^{-1} \boldsymbol{x}\right) \omega\left(U(\tau) E(\Delta) \alpha_{x}(C) E(\Delta) U(\tau)^{*}\right)\right| \\
& \quad \leqslant\|h\|_{\infty} \sup _{\phi \in \mathfrak{B}(\mathcal{H})_{*, 1}} \int_{\mathbb{R}^{s}} d^{s} x\left|\phi\left(E(\Delta) \alpha_{x}(C) E(\Delta)\right)\right|=\|h\|_{\infty} p_{\Delta}(C) .
\end{aligned}
$$

The above inequality implies that the functionals $\rho_{h, t}$ belong to the dual space $\mathfrak{C}^{*}$ of $\left(\mathfrak{C}, \mathfrak{T}_{p}\right)$. Moreover, the estimate (3.24) is uniform in $t$, so that the net $\left\{\rho_{h, t}: t \in \mathbb{R}\right\}$ is even an equicontinuous subset of $\mathfrak{C}^{*}$. The Theorem of Alaoğlu-Bourbaki [41, Theorem 8.5.2] then tells us, that this net is relatively compact with respect to the weak topology, leading to the following fundamental result.

Theorem 3.10 (Existence of Limits). Under the assumptions of Definition 3.9 the net $\left\{\rho_{h, t}: t \in \mathbb{R}\right\} \subseteq \mathfrak{C}_{\Delta}^{*}$ possesses weak limit points in $\mathfrak{C}^{*}$ at asymptotic times. This means that there exist functionals $\sigma_{h, \omega}^{(+)}$and $\sigma_{h, \omega}^{(-)}$on $\mathfrak{C}$ together with corresponding subnets $\left\{\rho_{h, t_{1}}: \imath \in J\right\}$ and $\left\{\rho_{h, t_{\kappa}}: \kappa \in K\right\}$, i.e. $\lim _{1} t_{1}=+\infty$ and $\lim _{\kappa} t_{\kappa}=-\infty$, such that for arbitrary $C \in \mathfrak{C}$

$$
\begin{aligned}
& \rho_{h, t_{\mathrm{l}}}(C) \underset{\mathrm{\imath}}{\longrightarrow} \sigma_{h, \omega}^{(+)}(C), \\
& \rho_{h, t_{\mathrm{\kappa}}}(C) \underset{\mathrm{\kappa}}{\longrightarrow} \sigma_{h, \omega}^{(-)}(C) .
\end{aligned}
$$

The heuristic picture laid open above suggests, that in theories which are reasonable from a physicist's point of view the net $\left\{\rho_{h, t}: t \in \mathbb{R}\right\}$ actually converges, but as yet we have not been able to give rigorously formulated conditions under which to prove this conjecture. This question seems to be connected with the problem of asymptotic completeness of quantum field theoretic models; one has to assure that in the limit of large times multiple scattering does no longer withhold the measurement results $\rho_{h, t}(C)$ from growing stable. Another possibility is the disappearance of the limit functionals $\sigma_{h, \omega}^{(+)}$and $\sigma_{h, \omega}^{(-)}$on all of the algebra of counters $\mathfrak{C}$, a phenomenon that we anticipate to encounter in theories without a particle interpretation (e.g. generalized free field). The denomination of the asymptotic functionals ' $\sigma$ ' is chosen to reflect their singular nature: the values that the functionals $\rho_{h, t}$ return for finite times $t$ when applied to the 
identity operator $\mathbf{1}$ (which is not contained in $\mathfrak{C}$ ) are divergent as $|t|^{s}$ at asymptotic times.

The convergence problem as yet only partially solved in the sense of Theorem 3.10, one can nevertheless establish a number of distinctive properties of the limit functionals $\sigma$ (from now on we will skip sub- and superscripts not to overburden the notation), that allow for their interpretation in terms of asymptotic configurations of particles. An immediate first consequence of the above construction is the following proposition.

Proposition 3.11 (Positivity and Continuity of Limits). Suppose that $\Delta$ is a bounded Borel subset of $\mathbb{R}^{s+1}, \omega \in \mathcal{S}(\Delta)$ a physical state of bounded energy and $h \in L^{\infty}\left(\mathbb{R}^{s}, d^{s} x\right)$ a non-negative function. Then the limit functionals $\sigma$ for the net $\left\{\rho_{h, t}: t \in \mathbb{R}\right\}$ are positive elements of $\mathfrak{C}_{\Delta}^{*}$ :

$$
\begin{aligned}
|\sigma(C)| & \leqslant\|h\|_{\infty} p_{\Delta}(C), \quad C \in \mathfrak{C} ; \\
0 & \leqslant \sigma(C), \quad C \in \mathfrak{C}^{+} .
\end{aligned}
$$

Remark. Due to the continuity of $\rho_{h, t}$ and $\sigma$ with respect to the $p_{\Delta}$-topology, these functionals can be continuously extended to $\overline{\mathfrak{C}}$ as well as $\mathfrak{A}_{\mathfrak{C}}$, where $\rho_{h, t}$ are explicitly given on $\mathfrak{A}_{\mathfrak{C}}$ by the formula (3.22) with $C \in \mathfrak{A}_{\mathfrak{C}}$. It is then easily established, by use of elements $C^{\prime}$ from $\mathfrak{C}$ lying in suitable $p_{\Delta}$-neighbourhoods of $C$, that the relations (3.25) remain valid on this larger subspace of the quasi-local algebra $\mathfrak{A}$.

The next result deals with the effect that space-time translations exert on these limit functionals. A further assumption on the velocity implementation $h \in L^{\infty}\left(\mathbb{R}^{s}, d^{s} v\right)$ turns out to be indispensible in their proof: $h$ has to be continuous, approximating a constant value in the limit $|\boldsymbol{v}| \rightarrow \infty$, i. e. $h-M_{h} \in C_{0}\left(\mathbb{R}^{s}\right)$ for a suitable constant $M_{h}$; these functions constitute a subspace of $C\left(\mathbb{R}^{s}\right)$ that will be denoted $C_{0, c}\left(\mathbb{R}^{s}\right)$ in the sequel.

Proposition 3.12 (Translation Invariance). Let $\Delta \subseteq \mathbb{R}^{s+1}$ be a bounded Borel set, let $\omega \in \mathcal{S}(\Delta)$ and $h \in C_{0, c}\left(\mathbb{R}^{s}\right)$. Then the limit functionals $\sigma$ of $\left\{\rho_{h, t_{1}}: 1 \in J\right\}$ are invariant under space-time translations:

$$
\sigma\left(\alpha_{x}(C)\right)=\sigma(C)
$$

for any $C \in \mathfrak{A}_{\mathfrak{C}}$ and any $x \in \mathbb{R}^{s+1}$.

Proof. Taking into account the fact that the Lebesgue measure on $\mathbb{R}^{s+1}$ is invariant under translations, one can express $\rho_{h, t}\left(\alpha_{\left(x^{0}, x\right)}(C)\right)$ for any finite time $t$ and any given $x=\left(x^{0}, \boldsymbol{x}\right) \in \mathbb{R}^{s+1}$ by the following integral

$$
\rho_{h, t}\left(\alpha_{\left(x^{0}, \boldsymbol{x}\right)}(C)\right)=T(t)^{-1} \int_{t+x^{0}}^{t+x^{0}+T(t)} d \tau \int_{\mathbb{R}^{s}} d^{s} y h\left(\left(\tau-x^{0}\right)^{-1}(\boldsymbol{y}-\boldsymbol{x})\right) \omega\left(\alpha_{(\tau, \boldsymbol{y})}(C)\right) .
$$

Next, we want to evaluate $\left|\rho_{h, t}(C)-\rho_{h, t}\left(\alpha_{\left(x^{0}, x\right)}(C)\right)\right|$ which, according to the respective limits of $\tau$-integration, can be split into a sum of three integrals to be estimated separately:

$$
\begin{aligned}
\left|T(t)^{-1} \int_{t}^{t+x^{0}} d \tau \int_{\mathbb{R}^{s}} d^{s} y h\left(\tau^{-1} \boldsymbol{y}\right) \omega\left(\alpha_{(\tau, \boldsymbol{y})}(C)\right)\right| & \leqslant|T(t)|^{-1}\left|x^{0}\right|\|h\|_{\infty} p_{\Delta}(C), \\
\left|T(t)^{-1} \int_{t+x^{0}+T(t)}^{t+T(t)} d \tau \int_{\mathbb{R}^{s}} d^{s} y h\left(\tau^{-1} \boldsymbol{y}\right) \omega\left(\alpha_{(\tau, \boldsymbol{y})}(C)\right)\right| & \leqslant|T(t)|^{-1}\left|x^{0}\right|\|h\|_{\infty} p_{\Delta}(C) ;
\end{aligned}
$$


both $\rho_{h, t}(C)$ and $\rho_{h, t}\left(\alpha_{\left(x^{0}, x\right)}(C)\right)$ contribute to the third integral

$$
\begin{array}{r}
\left|T(t)^{-1} \int_{t+x^{0}}^{t+x^{0}+T(t)} d \tau \int_{\mathbb{R}^{s}} d^{s} y\left[h\left(\tau^{-1} \boldsymbol{y}\right)-h\left(\left(\tau-x^{0}\right)^{-1}(\boldsymbol{y}-\boldsymbol{x})\right)\right] \omega\left(\alpha_{(\tau, \boldsymbol{y})}(C)\right)\right| \\
\leqslant \sup _{\tau \in I_{t, x^{0}}} \sup _{\boldsymbol{y} \in \mathbb{R}^{s}}\left|h\left(\tau^{-1} \boldsymbol{y}\right)-h\left(\left(\tau-x^{0}\right)^{-1}(\boldsymbol{y}-\boldsymbol{x})\right)\right| p_{\Delta}(C),
\end{array}
$$

where we used the abbreviation $I_{t, x^{0}}$ for the interval of $\tau$-integration. Setting (for $|\tau|$ large enough)

$$
z_{\tau} \doteq z+\left(\tau-x^{0}\right)^{-1}\left(x^{0} z-x\right)
$$

we finally arrive at the estimate

$$
\left|\rho_{h, t}(C)-\rho_{h, t}\left(\alpha_{\left(x^{0}, \boldsymbol{x}\right)}(C)\right)\right| \leqslant\left(2|T(t)|^{-1}\left|x^{0}\right|\|h\|_{\infty}+\sup _{\tau \in I_{t, x^{0}}} \sup _{z \in \mathbb{R}^{s}}\left|h(z)-h\left(z_{\tau}\right)\right|\right) p_{\Delta}(C) .
$$

The net $\left\{z_{\tau}: \tau \in \mathbb{R}\right\}$ approximates $z$ uniformly on compact subsets of $\mathbb{R}^{s}$ in the limit of large $|\tau|$, i. e. given $\varepsilon>0$ and $R^{\prime}>0$ there exists a positive number $T^{\prime}$ such that $|\tau|>T^{\prime}$ implies $\left|z-z_{\tau}\right|<\varepsilon$ for any $z \in \mathbb{R}^{s}$ with $|z| \leqslant R^{\prime}$. On the other hand, given $R^{\prime \prime}>0$ there exists $T^{\prime \prime}>0$ such that $\left|z_{\tau}\right|>\frac{1}{2} R^{\prime \prime}$ for any $|z|>R^{\prime \prime}$ and any $|\tau|>T^{\prime \prime}$. Combining these results with the special properties of $h \in C_{0, c}\left(\mathbb{R}^{s}\right)$, i. e. uniform continuity on compact balls in $\mathbb{R}^{s}$ and approximate constancy at infinity, we infer that for large $|\tau|$ the term $\sup _{z \in \mathbb{R}^{s}}\left|h(z)-h\left(z_{\tau}\right)\right|$ falls below any given positive bound. Therefore the right-hand side of (3.28) vanishes with $|t| \rightarrow \infty$ since $|T(t)|$ exceeds any positive value in this limit.

Now, let $\sigma$ be the weak limit of the subnet $\left\{\rho_{h, t_{l}}: \imath \in J\right\}$, i.e.

$$
\rho_{h, t_{l}}(C) \underset{\mathrm{l}}{\longrightarrow} \sigma(C)
$$

then there holds for any $\mathfrak{\imath} \in J$, any $C \in \mathfrak{A}_{\mathfrak{C}}$ and any $x \in \mathbb{R}^{s+1}$ the subsequent inequality

$$
\begin{aligned}
& 0 \leqslant\left|\sigma\left(\alpha_{x}(C)\right)-\sigma(C)\right| \\
& \quad \leqslant\left|\sigma\left(\alpha_{x}(C)\right)-\rho_{h, t_{l}}\left(\alpha_{x}(C)\right)\right|+\left|\rho_{h, t_{l}}\left(\alpha_{x}(C)\right)-\rho_{h, t_{\mathrm{t}}}(C)\right|+\left|\rho_{h, t_{\mathrm{L}}}(C)-\sigma(C)\right| .
\end{aligned}
$$

By the reasoning of the preceding paragraph and the above condition for subnet convergence, all three terms on the right-hand side vanish with respect to the directed set $J$, since in this limit $\left|t_{1}\right| \rightarrow \infty$. As a result the intermediate term has to be equal to 0 , thereby establishing translation invariance of $\sigma$.

The last property that we are going to demonstrate in this section for those special elements $\sigma \in \mathfrak{C}_{\Delta}{ }^{*+}$, that arise as limits of nets of functionals $\left\{\rho_{h, t_{l}}: \mathfrak{l} \in J\right\}$, complements the Cluster Property 3.6. It asserts, given certain specific operators $C \in \mathfrak{C}$, the existence of lower bounds for integrals of the functions $\boldsymbol{x} \mapsto \sigma\left(C^{*} \alpha_{x}(C)\right)$. 
Proposition 3.13 (Existence of Lower Bounds). Let $C \in \mathfrak{C}$ be a counter which has the property that the function $\boldsymbol{x} \mapsto p_{\Delta}\left(C^{*} \alpha_{x}(C)\right)$ is integrable (cf. Lemma 3.7). Let furthermore $\sigma \in \mathfrak{C}_{\Delta}{ }^{*+}$ be the limit of a net of functionals $\left\{\rho_{h, t_{l}}: \mathfrak{\imath} \in J\right\}$, each defined by (3.22), where the velocity function $h$ is non-negative and belongs to $C_{0, c}\left(\mathbb{R}^{s}\right)$. Under these assumptions

$$
|\sigma(C)|^{2} \leqslant\|h\|_{\infty} \int_{\mathbb{R}^{s}} d^{s} x \sigma\left(C^{*} \alpha_{x}(C)\right) .
$$

Proof. Consider the functional $\rho_{h, t}$ at finite time $t$. Applying to the absolute value of its defining equation (3.22) the Cauchy-Schwarz inequality with respect to the inner product $(|t|$ large enough)

$$
(f, g)_{t} \doteq T(t)^{-1} \int_{t}^{t+T(t)} d \tau \overline{f(\tau)} g(\tau)
$$

of square-integrable functions $f$ and $g$ depending on the time variable $\tau \in I_{t}$, one gets in the special case of

$$
f(\tau) \equiv 1 \quad \text { and } \quad g(\tau)=\int_{\mathbb{R}^{s}} d^{s} x h\left(\tau^{-1} \boldsymbol{x}\right) \omega\left(\alpha_{(\tau, x)}(C)\right)
$$

the estimate

$$
\begin{aligned}
& \left|\rho_{h, t}(C)\right|^{2}=\left|T(t)^{-1} \int_{t}^{t+T(t)} d \tau \int_{\mathbb{R}^{s}} d^{s} x h\left(\tau^{-1} \boldsymbol{x}\right) \omega\left(\alpha_{(\tau, \boldsymbol{x})}(C)\right)\right|^{2} \\
& \qquad T(t)^{-1} \int_{t}^{t+T(t)} d \tau\left|\int_{\mathbb{R}^{s}} d^{s} x h\left(\tau^{-1} \boldsymbol{x}\right) \omega\left(\alpha_{\tau}\left(\alpha_{x}(C)\right)\right)\right|^{2} .
\end{aligned}
$$

Now, let $\boldsymbol{K}$ be a compact subset of $\mathbb{R}^{s}$; then, by positivity of the functional $\omega \in \mathcal{S}(\Delta)$, [11, Proposition 2.3.11(b)] together with the Fubini Theorem [26, II.16.3] leads for arbitrary $\tau \in \mathbb{R}$ to

$$
\begin{aligned}
&\left|\omega\left(\alpha_{\tau}\left(\int_{\boldsymbol{K}} d^{s} x h\left(\tau^{-1} \boldsymbol{x}\right) \alpha_{x}(C)\right)\right)\right|^{2} \\
& \leqslant \omega\left(\alpha_{\tau}\left(\int_{\boldsymbol{K}} d^{s} x \int_{\boldsymbol{K}} d^{s} y h\left(\tau^{-1} \boldsymbol{y}\right) h\left(\tau^{-1} \boldsymbol{x}\right) \alpha_{y}\left(C^{*}\right) \alpha_{x}(C)\right)\right),
\end{aligned}
$$

which is preserved in the limit $K \nearrow \mathbb{R}^{s}$, which exists on account of the assumed integrability of the mapping $\boldsymbol{x} \mapsto p_{\Delta}\left(C^{*} \alpha_{x}(C)\right)$. On commuting $\omega \circ \alpha_{\tau}$ and the integrals one arrives at

$$
\begin{aligned}
& \left|\int_{\mathbb{R}^{s}} d^{s} x h\left(\tau^{-1} \boldsymbol{x}\right) \omega\left(\alpha_{\tau}\left(\alpha_{x}(C)\right)\right)\right|^{2} \\
& \leqslant \int_{\mathbb{R}^{s}} d^{s} x \int_{\mathbb{R}^{s}} d^{s} y h\left(\tau^{-1} \boldsymbol{y}\right) h\left(\tau^{-1} \boldsymbol{x}\right) \omega\left(\alpha_{\tau}\left(\alpha_{y}\left(C^{*}\right) \alpha_{x}(C)\right)\right) \leqslant\|h\|_{\infty}^{2} \int_{\mathbb{R}^{s}} d^{s} x p_{\Delta}\left(C^{*} \alpha_{x}(C)\right)
\end{aligned}
$$

and the combination of (3.30) and (3.31) gives

$$
\left|\rho_{h, t}(C)\right|^{2} \leqslant T(t)^{-1} \int_{t}^{t+T(t)} d \tau \int_{\mathbb{R}^{s}} d^{s} x \int_{\mathbb{R}^{s}} d^{s} y h\left(\tau^{-1} \boldsymbol{y}\right) h\left(\tau^{-1} \boldsymbol{x}\right) \omega\left(\alpha_{\tau}\left(\alpha_{\boldsymbol{y}}\left(C^{*}\right) \alpha_{x}(C)\right)\right) .
$$


We want to replace the term $h\left(\tau^{-1} \boldsymbol{x}\right)$ by the norm $\|h\|_{\infty}$ and, to do so, define the function $h_{+} \doteq\left(\|h\|_{\infty} h-h^{2}\right)^{1 / 2}$, which is a non-negative element of $C_{0, c}\left(\mathbb{R}^{s}\right)$ as is $h$ itself. Then for any $z, z^{\prime} \in \mathbb{R}^{s}$ there holds the equation

$$
\|h\|_{\infty} h(z)=h(z) h\left(z^{\prime}\right)+h_{+}(z) h_{+}\left(z^{\prime}\right)+h_{+}(z)\left(h_{+}(z)-h_{+}\left(z^{\prime}\right)\right)+h(z)\left(h(z)-h\left(z^{\prime}\right)\right) .
$$

Next, consider for an arbitrary function $g \in C_{0, c}\left(\mathbb{R}^{s}\right)$ the following inequality, based on an application of Fubini's Theorem and the reasoning of (3.24),

$$
\begin{array}{r}
\left|T(t)^{-1} \int_{t}^{t+T(t)} d \tau \int_{\mathbb{R}^{s}} d^{s} x \int_{\mathbb{R}^{s}} d^{s} y g\left(\tau^{-1} \boldsymbol{y}\right)\left(g\left(\tau^{-1} \boldsymbol{y}\right)-g\left(\tau^{-1} \boldsymbol{x}\right)\right) \omega\left(\alpha_{\tau}\left(\alpha_{\boldsymbol{y}}\left(C^{*}\right) \boldsymbol{\alpha}_{\boldsymbol{x}}(C)\right)\right)\right| \\
=\left|\int_{\mathbb{R}^{s}} d^{s} x T(t)^{-1} \int_{t}^{t+T(t)} d \tau \int_{\mathbb{R}^{s}} d^{s} z \tau^{s} g(\boldsymbol{z})\left(g(\boldsymbol{z})-g\left(\boldsymbol{z}_{\tau}(\boldsymbol{x})\right)\right) \omega\left(\boldsymbol{\alpha}_{(\tau, \tau z)}\left(C^{*} \boldsymbol{\alpha}_{\boldsymbol{x}}(C)\right)\right)\right| \\
\quad \leqslant\|g\|_{\infty} \int_{\mathbb{R}^{s}} d^{s} x \sup _{\tau \in I_{t}} \sup _{\boldsymbol{z} \in \mathbb{R}^{s}}\left|g(\boldsymbol{z})-g\left(\boldsymbol{z}_{\tau}(\boldsymbol{x})\right)\right| p_{\Delta}\left(C^{*} \alpha_{\boldsymbol{x}}(C)\right), \quad \text { (3.34) }
\end{array}
$$

where we made use of the coordinate transformation $\boldsymbol{x} \rightsquigarrow \boldsymbol{x}+\boldsymbol{y}$ followed by the transformation $\boldsymbol{y} \rightsquigarrow \boldsymbol{z} \doteq \tau^{-1} \boldsymbol{y}$ and introduced the abbreviations $\boldsymbol{z}_{\tau}(\boldsymbol{x}) \doteq \tau^{-1} \boldsymbol{x}+\boldsymbol{z}$ as well as $I_{t}$ for the interval of $\tau$-integration. Similar to the proof of Proposition 3.12, the expression $\sup _{\tau \in I_{t}} \sup _{z \in \mathbb{R}^{s}}\left|g(\boldsymbol{z})-g\left(\boldsymbol{z}_{\tau}(\boldsymbol{x})\right)\right|$ is seen to vanish for all $\boldsymbol{x} \in \mathbb{R}^{s}$ in the limit of large $|t|$, so that by Lebesgue's Dominated Convergence Theorem the left-hand side of (3.34) converges to 0 . This reasoning in particular applies to the functions $h$ as well as $h_{+}$and thus to the third and fourth term on the right of equation (3.33). On the other hand, substitution of $h$ by $h_{+}$in the integral of (3.32) likewise gives a non-negative result for all times $t$. Combining all these informations and specializing to a subnet $\left\{t_{1}: l \in J\right\}$ approximating $+\infty$ or $-\infty$, one arrives at the following version of (3.32), valid for asymptotic times:

$$
\begin{aligned}
& \lim _{\imath}\left|\rho_{h, t_{l}}(C)\right|^{2} \\
& \leqslant \lim _{\mathrm{l}}\|h\|_{\infty} T\left(t_{\mathrm{l}}\right)^{-1} \int_{t_{\mathrm{l}}}^{t_{\mathrm{l}}+T\left(t_{\mathrm{l}}\right)} d \tau \int_{\mathbb{R}^{s}} d^{s} x \int_{\mathbb{R}^{s}} d^{s} y h\left(\tau^{-1} \boldsymbol{y}\right) \omega\left(\alpha_{\tau}\left(\alpha_{y}\left(C^{*}\right) \alpha_{x}(C)\right)\right) \\
& \leqslant\|h\|_{\infty} \lim _{\imath} \int_{\mathbb{R}^{s}} d^{s} x T\left(t_{\imath}\right)^{-1} \int_{t_{\imath}}^{t_{\imath}+T\left(t_{\imath}\right)} d \tau \int_{\mathbb{R}^{s}} d^{s} y h\left(\tau^{-1} \boldsymbol{y}\right) \omega\left(\alpha_{(\tau, \boldsymbol{y})}\left(C^{*} \alpha_{x}(C)\right)\right) \\
& =\|h\|_{\infty} \lim _{\imath} \int_{\mathbb{R}^{s}} d^{s} x \rho_{h, t_{\iota}}\left(C^{*} \alpha_{x}(C)\right) .
\end{aligned}
$$

Making use of Lemma 3.7, this result can be expressed in terms of the functional $\sigma=$ $\lim _{\imath} \rho_{h, t_{l}}$ to yield

$$
|\sigma(C)|^{2} \leqslant\|h\|_{\infty} \int_{\mathbb{R}^{s}} d^{s} x \sigma\left(C^{*} \alpha_{x}(C)\right)
$$

\subsection{Particle Weights}

The features of limit functionals $\sigma \in \mathfrak{C}_{\Delta}{ }^{*+}$ collected thus far, point to their interpretation as representatives of mixtures of particle-like quantities with sharp energy-momentum: 
being translationally invariant according to Proposition 3.12, they appear as plane waves, i. e. energy-momentum eigenstates, on the other hand they are singly localized at all times by Proposition 3.6, thereby exhibiting properties of particle-like systems, their energy-momentum spectrum being determined by Proposition 3.8. We shall summarize systems of the above kind under the concept of particle weights, a term chosen to reflect the connection to the notion of 'weights' or 'extended positive functionals' in the theory of $C^{*}$-algebras, going back to Dixmier [24, Section I.4.2] (cf. also [48, Section 5.1] and [47]). These designate functions on the positive cone $\mathfrak{A}^{+}$of a $C^{*}$-algebra $\mathfrak{A}$ which can attain infinite values, a property they share with the singular functionals constructed in Theorem 3.10: it was seen to be of importance that their domain $\mathfrak{C}$ does not comprise the element $\mathbf{1}$ of the quasi-local algebra, for the defining approximation would then lead to the value $\sigma(\mathbf{1})=+\infty$.

As already mentioned in Section 3.1, every positive functional $\sigma$ on $\mathfrak{C}=\mathfrak{L}^{*} \mathfrak{L}$ defines a non-negative sesquilinear form $\langle. \mid .\rangle_{\sigma}$ on $\mathfrak{L} \times \mathfrak{L}$ via

$$
\left\langle L_{1} \mid L_{2}\right\rangle_{\sigma} \doteq \sigma\left(L_{1}^{*} L_{2}\right)
$$

for any $L_{1}, L_{2} \in \mathfrak{L}$, which induces a seminorm $q_{\sigma}$ on $\mathfrak{L}$ and a norm \|\|$_{\sigma}$ on the corresponding quotient of $\mathfrak{L}$ by the null space $\mathfrak{N}_{\sigma}$ of $q_{\sigma}$. Taking advantage of these constructions, we shall depart from functionals and proceed to sesquilinear forms, a step which is necessitated by the special demands of the subsequent analysis. The following definition consists of a résumé of the essence of our knowledge on asymptotic functionals acquired in the above sequence of propositions.

Definition 3.14. A particle weight is a non-trivial, non-negative sesquilinear form on $\mathfrak{L}$, written $\langle. \mid$.$\rangle , which induces by (3.2) on the ideal \mathfrak{L}$ a seminorm $q_{w}$ with null space $\mathfrak{N}_{w}$ as well as a norm $\|.\|_{w}$ on the quotient $\mathfrak{L} / \mathfrak{N}_{w}$, and which complies with the following assumptions:

(i) for any $L_{1}, L_{2} \in \mathfrak{L}$ and $A \in \mathfrak{A}$ there holds the relation

$$
\left\langle L_{1} \mid A L_{2}\right\rangle=\left\langle A^{*} L_{1} \mid L_{2}\right\rangle
$$

(ii) for given $L \in \mathfrak{L}$ the following mapping is continuous with respect to $q_{w}$ :

$$
\Xi_{L}: P_{+}^{\uparrow} \rightarrow \mathfrak{L} \quad(\Lambda, x) \mapsto \Xi_{L}(\Lambda, x)=\alpha_{(\Lambda, x)}(L) ;
$$

(iii) the restriction to the subspace $\mathfrak{L}_{0}$ of the canonical homomorphism

$$
\mathcal{Q}_{w}: \mathfrak{L} \rightarrow \mathfrak{L} / \mathfrak{N}_{w} \quad L \mapsto Q_{w}(L) \doteq[L]_{w}
$$

is $X_{\mathfrak{L}_{0}}$-differentiable in the sense of Definition A.16;

(iv) the sesquilinear form is invariant with respect to space-time translations $x \in \mathbb{R}^{s+1}$, i.e.

$$
\left\langle\alpha_{x}\left(L_{1}\right) \mid \alpha_{x}\left(L_{2}\right)\right\rangle=\left\langle L_{1} \mid L_{2}\right\rangle, \quad L_{1}, L_{2} \in \mathfrak{L},
$$

and the $(s+1)$-dimensional Fourier transforms of the distributions

$$
x \mapsto\left\langle L_{1} \mid \alpha_{x}\left(L_{2}\right)\right\rangle
$$

have support in a shifted forward light cone $\bar{V}_{+}-q$, where $q \in \bar{V}_{+}$. 
Particle Weights as Asymptotic Plane Waves

Remark. (i) Note, that we did not impose on $\langle. \mid$.$\rangle any restrictions concerning con-$ tinuity with respect to the $q_{\Delta}$-topology of $\mathfrak{L}$, for in general such conditions will get lost in the disintegration of particle weights to be expounded in Chapter 4. The continuity property, which actually depends on the topology of $\mathfrak{L}$, is formulated in terms of the seminorm $q_{w}$ induced by the sesquilinear form under consideration. The constituent properties of the above definition are preserved under the operations of addition and of multiplication by positive numbers, so that the totality of particle weights supplemented by the trivial form proves to be a positive (proper convex) cone (cf. [49, 5]), denoted $W$, in the linear space of all sesquilinear forms on $\mathfrak{L}$. This ascertainment is the foundation for the constructions of Chapter 6 .

(ii) One could be tempted to go the way back from a sesquilinear form of the above type to a positive linear functional on $\mathfrak{C}$, but this is by no means self-evident. It is only possible under restrictive assumptions on the structure of the algebra $\mathfrak{C}$ to make the definition of the associated functional unambiguous.

A completely equivalent characterization of particle weights can be given in terms of representations $\left(\pi_{w}, \mathcal{H}_{w}\right)$ of the quasi-local algebra $\mathfrak{A}$, obtained by means of a GNSconstruction (cf. [47, Theorem 3.2] and [48, Proposition 5.1.3]).

Theorem 3.15. (I) To any particle weight $\langle. \mid$.$\rangle there corresponds a non-zero, non-$ degenerate representation $\left(\pi_{w}, \mathcal{H}_{w}\right)$ of the quasi-local $C^{*}$-algebra $\mathfrak{A}$ with the following properties:

(i) there exists a linear mapping $|$.$\rangle from \mathfrak{L}$ onto a dense subspace of $\mathcal{H}_{w}$

$$
|.\rangle: \mathfrak{L} \rightarrow \mathcal{H}_{w} \quad L \mapsto|L\rangle,
$$

such that the representation $\pi_{w}$ is given by

$$
\pi_{w}(A)|L\rangle=|A L\rangle, \quad A \in \mathfrak{A}, \quad L \in \mathfrak{L} ;
$$

(ii) the following mapping is continuous for given $L \in \mathfrak{L}$ :

$$
\left|\Xi_{L}(.)\right\rangle: \mathrm{P}_{+}^{\uparrow} \rightarrow \mathcal{H}_{w} \quad(\Lambda, x) \mapsto\left|\Xi_{L}(\Lambda, x)\right\rangle=\left|\alpha_{(\Lambda, x)}(L)\right\rangle ;
$$

(iii) the restriction of the linear mapping $|$.$\rangle to \mathfrak{L}_{0}$ with range in the subspace of $\mathcal{H}_{w}$ spanned by all vectors $\left|L_{0}\right\rangle, L_{0} \in \mathfrak{L}_{0}$, is $\mathcal{X}_{\mathfrak{L}_{0}}$-differentiable;

(iv) there exists a strongly continuous unitary representation $x \mapsto U_{w}(x)$ of space-time translations $x \in \mathbb{R}^{s+1}$ on $\mathcal{H}_{w}$ defined by

$$
U_{w}(x)|L\rangle \doteq\left|\alpha_{x}(L)\right\rangle, \quad L \in \mathfrak{L},
$$

with spectrum in a displaced forward light cone $\bar{V}_{+}-q, q \in \bar{V}_{+}$.

(II) Any representation $\left(\pi_{w}, \mathcal{H}_{w}\right)$ which has the above characteristics defines a particle weight through the scalar product on $\mathcal{H}_{w}$.

Remark. By their very definition, the unitaries $U_{w}(x)$ implement the automorphism group $\left\{\alpha_{x}: x \in \mathbb{R}^{s+1}\right\} \subseteq$ Aut $\mathfrak{A}$ through

$$
U_{w}(x) \pi_{w}(A) U_{w}(x)^{*}=\pi_{w}\left(\alpha_{x}(A)\right), \quad A \in \mathfrak{A}, x \in \mathbb{R}^{s+1},
$$

in the representation $\left(\mathcal{H}_{w}, \pi_{w}\right)$. 
Proof. Part (I): The proof of the various properties stated in the Theorem is readily carried out, once the GNS-construction has been realized.

(i) Since a particle weight satisfies the Cauchy-Schwarz inequality its null space

$$
\mathfrak{N}_{w} \doteq\{N \in \mathfrak{L}:\langle N \mid N\rangle=0\}
$$

turns out to be a left ideal in $\mathfrak{L}$ (and hence in $\mathfrak{A}$ ). The defining sesquilinear form endows the quotient space of $\mathfrak{L}$ by $\mathfrak{N}_{w}$ with a pre-Hilbert space structure; its completion $\mathcal{H}_{w}$ contains by construction the range of the canonical homomorphism

$$
|.\rangle: \mathfrak{L} \rightarrow \mathfrak{L} / \mathfrak{N}_{w} \quad L \mapsto|L\rangle \doteq[L]_{w}
$$

as a dense subspace. $\mathfrak{L}$ and $\mathfrak{N}_{w}$ being left ideals in $\mathfrak{A}$, the definition

$$
\pi_{w}(A)|L\rangle \doteq|A L\rangle, \quad A \in \mathfrak{A},
$$

makes sense on the range of $|$.$\rangle and can be extended to all of \mathcal{H}_{w}$ due to the estimate

$$
\| \pi_{w}(A)|L\rangle\left\|^{2}=\langle A L \mid A L\rangle=\left\langle L \mid A^{*} A L\right\rangle \leqslant\right\| A\left\|^{2}\langle L \mid L\rangle=\right\| A\left\|^{2}\right\||L\rangle \|^{2},
$$

which is founded on the fact that the particle weight is a non-negative sesquilinear form and the operator $\|A\|^{2} \mathbf{1}-A^{*} A$ is positive. Since $\mathfrak{A}$ is unital, this yields a non-zero, nondegenerate representation of the quasi-local algebra on the Hilbert space $\mathcal{H}_{w}$.

(ii) The norm on $\mathcal{H}_{w}$ induces a seminorm on $\mathfrak{L}$ via the linear mapping $|$.$\rangle and this$ coincides with $q_{w}$ as defined for particle weights. Therefore the asserted continuity of the mapping $(\Lambda, x) \mapsto\left|\alpha_{(\Lambda, x)}(L)\right\rangle$ is an immediate consequence of the respective property in Definition 3.14.

(iii) By construction, the canonical homomorphisms $|$.$\rangle and Q_{w}$ coincide and furthermore $\||L\rangle\|=\|[L]_{w} \|_{w}$, so that the assumption of $X_{\mathfrak{L}_{0}}$-differentiability is self-evident.

(iv) The existence of a strongly continuous unitary representation of space-time translations in $\left(\pi_{w}, \mathcal{H}_{w}\right)$ is a direct consequence of translation invariance of the particle weight $\langle. \mid$.$\rangle and its continuity under Poincaré transformations with respect to q_{w}$. Stone's Theorem (cf. [6, Chapter 6, §2] and [38, Theorem VIII.(33.8)]) connects the spectrum of its generator $P_{w}=\left(P_{w}^{\mu}\right)$ to the support of the Fourier transform of $x \mapsto\left\langle L_{1} \mid \alpha_{x}\left(L_{2}\right)\right\rangle$ in Definition 3.14 by virtue of the relation

$$
\begin{aligned}
\int_{\mathbb{R}^{s+1}} d^{s+1} x g(x)\left\langle L_{1} \mid \alpha_{x}\left(L_{2}\right)\right\rangle=\int_{\mathbb{R}^{s+1}} d^{s+1} x g(x) & \left\langle L_{1}\left|U_{w}(x)\right| L_{2}\right\rangle \\
& =(2 \pi)^{(s+1) / 2}\left\langle L_{1}\left|\tilde{g}\left(P_{w}\right)\right| L_{2}\right\rangle,
\end{aligned}
$$

which holds for any $L_{1}, L_{2} \in \mathfrak{L}$ and any $g \in L^{1}\left(\mathbb{R}^{s+1}, d^{s+1} x\right)$. To clarify this fact, note, that the projection-valued measure $E_{w}($.$) corresponding to P_{w}$ is regular, i. e. $E_{w}\left(\Delta^{\prime}\right)$ is for any Borel set $\Delta^{\prime}$ the strong limit of the net $\left\{E_{w}\left(\Gamma^{\prime}\right): \Gamma^{\prime} \subseteq \Delta^{\prime}\right.$ compact $\}$. For each compact $\Gamma \subseteq \complement\left(\bar{V}_{+}-q\right)$ consider an infinitely often differentiable function $\tilde{g}_{\Gamma}$ with support in $\complement\left(\bar{V}_{+}-q\right)$ that envelops the characteristic function for $\Gamma$ (cf. [40, Satz 7.7]): $0 \leqslant \chi_{\Gamma} \leqslant \tilde{g}_{\Gamma}$. According to the assumption of Definition 3.14 the left-hand side of (3.38) vanishes for any $g_{\Gamma}$ of the above kind, and this means that all the bounded operators $\tilde{g}_{\Gamma}\left(P_{w}\right)$ equal 0 not only on the dense subspace spanned by vectors $|L\rangle, L \in \mathfrak{L}$, but on all of $\mathcal{H}_{w}$. Due to the fact that $\tilde{g}_{\Gamma}$ majorizes $\chi_{\Gamma}$, this in turn implies $\chi_{\Gamma}\left(P_{w}\right)=E_{w}(\Gamma)=0$ and thus, by arbitrariness of $\Gamma \subseteq \complement\left(\bar{V}_{+}-q\right)$ in connection with regularity, the desired relation $E_{w}\left(\complement\left(\bar{V}_{+}-q\right)\right)=0$. 
Particle Weights as Asymptotic Plane Waves

Part (II): The reversion of the above arguments in order to establish that the scalar product on $\mathcal{H}_{w}$ possesses the characteristics of a particle weight is self-evident.

The following analogue of Lemmas 2.17 and 2.18 in terms of the $q_{w}$-topology induced on $\mathfrak{L}$ by a particle weight is of importance not only for the remaining results of this chapter, but plays an important role in the constructions that underlie the theory of disintegration to be expounded in Chapter 4.

Lemma 3.16. Let $L \in \mathfrak{L}$ and let $\langle. \mid$.$\rangle be a particle weight.$

(i) Let $F \in L^{1}\left(\mathrm{P}_{+}^{\uparrow}, d \mu(\Lambda, x)\right)$ have compact support $\mathrm{S}$, then the Bochner integral

$$
\alpha_{F}(L)=\int d \mu(\Lambda, x) F(\Lambda, x) \alpha_{(\Lambda, x)}(L)
$$

lies in the completion of $\mathfrak{L}$ with respect to the locally convex topology induced on it by the initial norm $\|$.$\| and the q_{w}$-seminorm defined by the particle weight. Moreover $\left|\alpha_{F}(L)\right\rangle$ is a vector in the corresponding Hilbert space $\mathcal{H}_{w}$ and can be written

$$
\left|\alpha_{F}(L)\right\rangle=\int d \mu(\Lambda, x) F(\Lambda, x)\left|\alpha_{(\Lambda, x)}(L)\right\rangle,
$$

satisfying the inequality

$$
\|\left|\alpha_{F}(L)\right\rangle\|\leqslant\| F\left\|_{1} \sup _{(\Lambda, x) \in \mathrm{S}}\right\|\left|\alpha_{(\Lambda, x)}(L)\right\rangle \| .
$$

(ii) For any function $g \in L^{1}\left(\mathbb{R}^{s+1}, d^{s+1} x\right)$ the Bochner integral

$$
\alpha_{g}(L)=\int_{\mathbb{R}^{s+1}} d^{s+1} x g(x) \alpha_{x}(L)
$$

likewise lies in the completion of $\mathfrak{L}$ with respect to the locally convex topology mentioned above. $\left|\alpha_{g}(L)\right\rangle$ is a vector in the Hilbert space $\mathcal{H}_{w}$ subject to the relation

$$
\left|\alpha_{g}(L)\right\rangle=\int_{\mathbb{R}^{s+1}} d^{s+1} x g(x)\left|\alpha_{x}(L)\right\rangle=(2 \pi)^{(s+1) / 2} \tilde{g}\left(P_{w}\right)|L\rangle,
$$

so that

$$
\|\left|\alpha_{g}(L)\right\rangle\|\leqslant\| g\left\|_{1}\right\||L\rangle \|
$$

Proof. (i) Due to continuity of the particle weight $\langle$.$| . \rangle$ with respect to Poincaré transformations as claimed in Definition 3.14, the integrand of (3.39a) can be estimated with respect to the seminorm $q_{w}$ induced on $\mathfrak{L}$, which gives the Lebesgue-integrable function $(\Lambda, x) \mapsto|F(\Lambda, x)| \cdot \sup _{(\Lambda, x) \in \mathrm{S}} q_{w}\left(\alpha_{(\Lambda, x)}(L)\right)$. Therefore the integral in question indeed exists in the completion of the locally convex space $\mathfrak{L}$ not only with respect to the norm topology but also with respect to the seminorm $q_{w}$. Furthermore the corresponding GNS-construction of $\left(\pi_{w}, \mathcal{H}_{w}\right)$ implies that $\||L\rangle \|$ coincides with $q_{w}(L)$ for any $L \in \mathfrak{L}$, a relation which extends to the respective completions (cf. [44, Chapter One, § 54.(4)]) thus resulting in (3.39b). (3.39c) is then an immediate consequence, again on grounds of continuity under Poincaré transformations. 
(ii) According to Definition 3.14, the particle weight $\langle. \mid$.$\rangle is invariant under space-$ time translations and so is the seminorm $q_{w}$. Therefore the integrand of (3.40a) is majorized by the Lebesgue-integrable function $x \mapsto|g(x)| q_{w}(L)$, so that the respective integral exists in the completion of $\mathfrak{L}$. The first equation of (3.40b) arises from the same arguments that were already applied above, whereas the second one is then a consequence of Stone's Theorem (cf. (3.38)). Again on the ground of translation invariance, the estimate (3.40c) is an immediate conclusion from (3.40b).

Having this preparatory result at our disposal, we are in the position to prove a statement on spectral subspaces of $\mathcal{H}_{w}$, that will be significant in the next chapter as well as for the subsequent proof of the Cluster Property for particle weights.

Proposition 3.17 (Spectral Subspaces). Let L be an element of $\mathfrak{L}\left(\Delta^{\prime}\right)=\mathfrak{L} \cap \widetilde{\mathfrak{A}}\left(\Delta^{\prime}\right)$, which means that $L \in \mathfrak{L}$ has energy-momentum transfer in the Borel subset $\Delta^{\prime}$ of $\mathbb{R}^{s+1}$. Then, in the representation $\left(\pi_{w}, \mathcal{H}_{w}\right)$ corresponding to the particle weight $\langle. \mid$.$\rangle , the$ vector $|L\rangle$ belongs to the spectral subspace which pertains to $\Delta^{\prime}$ with respect to the intrinsic unitary representation $x \mapsto U_{w}(x)$ of space-time translations:

$$
|L\rangle=E_{w}\left(\Delta^{\prime}\right)|L\rangle .
$$

Proof. The energy-momentum transfer of an operator $A \in \mathfrak{A}$ can be stated in terms of the support properties of the Fourier transform of the mapping $x \mapsto \alpha_{x}(A)$ considered as an operator-valued distribution (cf. the remark following Definition 2.2). For the operator $L \in \mathfrak{L}\left(\Delta^{\prime}\right)$ this has the consequence that $\alpha_{g}(L)=0$ if $g$ is any Lebesgue-integrable function with supp $\tilde{g} \cap \Delta^{\prime}=\emptyset$. In this case we have, by an application of Lemma 3.16,

$$
\int_{\mathbb{R}^{s+1}} d^{s+1} x g(x)\left|\alpha_{x}(L)\right\rangle=\left|\alpha_{g}(L)\right\rangle=0 .
$$

Upon insertion of (3.42) into the formulation (3.38) of Stone's Theorem, the reasoning applied in the proof of Theorem 3.15 yields the assertion.

The particle weights enjoy a Cluster Property parallel to that established in Proposition 3.6 for functionals in $\mathfrak{C}_{\Delta}{ }^{*+}$. This characteristic, shared by the asymptotic functionals $\sigma$, could have been included in Definition 3.14, but it turns out, that it is already enforced by the other features.

Proposition 3.18 (Cluster Property for Particle Weights). Let $L_{i}$ and $L_{i}^{\prime}$ be elements of $\mathfrak{L}_{0}$ with energy-momentum transfer $\Gamma_{i}$ respectively $\Gamma_{i}^{\prime}$, and let $A_{i} \in \mathfrak{A}, i=1,2$, be almost local operators. Suppose furthermore that $\langle. \mid$.$\rangle is a particle weight with$ associated GNS-representation $\left(\pi_{w}, \mathcal{H}_{w}\right)$, then

$$
\mathbb{R}^{s} \ni \boldsymbol{x} \mapsto\left\langle L_{1}{ }^{*} A_{1} L_{1}^{\prime} \mid \alpha_{\boldsymbol{x}}\left(L_{2}{ }^{*} A_{2} L_{2}^{\prime}\right)\right\rangle=\left\langle L_{1}{ }^{*} A_{1} L_{1}^{\prime}\left|U_{w}(\boldsymbol{x})\right| L_{2}{ }^{*} A_{2} L_{2}^{\prime}\right\rangle \in \mathbb{C}
$$

is a function in $L^{1}\left(\mathbb{R}^{s}, d^{s} x\right)$.

Proof. To establish this result we follow in the main the strategy of the proof of Proposition 3.6. Applied to the problem at hand in terms of $\left(\pi_{w}, \mathcal{H}_{w}\right)$, this yields initially 
the estimate

$$
\begin{aligned}
& \left|\left\langle L_{1}{ }^{*} A_{1} L_{1}^{\prime}\left|U_{w}(\boldsymbol{x})\right| L_{2}{ }^{*} A_{2} L_{2}^{\prime}\right\rangle\right| \\
& \quad \leqslant\left|\left\langle L_{1}^{\prime}\left|\pi_{w}\left(\left[A_{1}{ }^{*} L_{1}, \alpha_{x}\left(L_{2}{ }^{*} A_{2}\right)\right]\right) U_{w}(\boldsymbol{x})\right| L_{2}^{\prime}\right\rangle\right|+\left|\left\langle L_{1}^{\prime}\left|\pi_{w}\left(\alpha_{x}\left(L_{2}{ }^{*} A_{2}\right) A_{1}{ }^{*} L_{1}\right) U_{w}(\boldsymbol{x})\right| L_{2}^{\prime}\right\rangle\right|
\end{aligned}
$$

for any $\boldsymbol{x} \in \mathbb{R}^{s}$. The first term on the right-hand side turns out to be majorized by $\left\|\left[A_{1}{ }^{*} L_{1}, \alpha_{x}\left(L_{2}{ }^{*} A_{2}\right)\right]\right\| \|\left|L_{1}^{\prime}\right\rangle\|\|\left|L_{2}^{\prime}\right\rangle \|$ in view of the fact that the particle weight is invariant under translations and that the representation $\pi_{w}$ is continuous. As the operators involved are almost local without exception, the norm of the commutator taking part in this expression decreases rapidly, thus rendering it integrable. The second term requires a closer inspection. One has

$$
\begin{aligned}
& 2\left|\left\langle L_{1}^{\prime}\left|\pi_{w}\left(\alpha_{x}\left(L_{2}{ }^{*} A_{2}\right) A_{1}{ }^{*} L_{1}\right) U_{w}(\boldsymbol{x})\right| L_{2}^{\prime}\right\rangle\right| \\
& \leqslant 2 \| \pi_{w}\left(\alpha_{x}\left(A_{2}{ }^{*} L_{2}\right)\right)\left|L_{1}^{\prime}\right\rangle\|\| \pi_{w}\left(A_{1}{ }^{*} L_{1}\right) U_{w}(\boldsymbol{x})\left|L_{2}^{\prime}\right\rangle \| \\
& \leqslant \| \pi_{w}\left(\alpha_{x}\left(A_{2}{ }^{*} L_{2}\right)\right)\left|L_{1}^{\prime}\right\rangle\left\|^{2}+\right\| \pi_{w}\left(\alpha_{(-x)}\left(A_{1}{ }^{*} L_{1}\right)\right)\left|L_{2}^{\prime}\right\rangle \|^{2},
\end{aligned}
$$

again by translation invariance of the particle weight in the last estimate. Now, a substitute of Lemma 3.5 has to be sought for, which was applied in the proof of Proposition 3.6 to get an estimate for (3.12), corresponding to the right-hand side of (3.44). Note, that $\pi_{w}\left(A^{\prime}\right)$ has the same energy-momentum transfer with respect to the unitary group $\left\{U_{w}(x): x \in \mathbb{R}^{s+1}\right\}$ as the operator $A^{\prime} \in \mathfrak{A}$ has regarding the underlying positive energy representation, and that, according to Proposition 3.17, $\left|L_{1}^{\prime}\right\rangle=E_{w}\left(\Gamma_{1}^{\prime}\right)\left|L_{1}^{\prime}\right\rangle$ and $\left|L_{2}^{\prime}\right\rangle=E_{w}\left(\Gamma_{2}^{\prime}\right)\left|L_{2}^{\prime}\right\rangle$ belong to the spectral subspaces pertaining to the compact sets $\Gamma_{1}^{\prime}$ and $\Gamma_{2}^{\prime}$. As in addition the spectrum of $\left\{U_{w}(x): x \in \mathbb{R}^{s+1}\right\}$ is restricted to a displaced forward light cone, all of the arguments given in the proofs of Propositions 2.6 and 2.7 also apply to the representation $\left(\pi_{w}, \mathcal{H}_{w}\right)$, so that e. $g$.

$$
\int_{\mathbb{R}^{s}} d^{s} x E_{w}\left(\Gamma_{1}^{\prime}\right) \pi_{w}\left(\alpha_{x}\left(L_{2}^{*} A_{2} A_{2}^{*} L_{2}\right)\right) E_{w}\left(\Gamma_{1}^{\prime}\right)
$$

is seen to exist in the $\sigma$-weak-topology on $\mathfrak{B}\left(\mathcal{H}_{w}\right)$. For this term we thus have

$$
\begin{aligned}
& \int_{\mathbb{R}^{s}} d^{s} x \| \pi_{w}\left(\alpha_{x}\left(A_{2}{ }^{*} L_{2}\right)\right) E_{w}\left(\Gamma_{1}^{\prime}\right)\left|L_{1}^{\prime}\right\rangle \|^{2} \\
&=\int_{\mathbb{R}^{s}} d^{s} x\left\langle L_{1}^{\prime}\left|E_{w}\left(\Gamma_{1}^{\prime}\right) \pi_{w}\left(\alpha_{x}\left(L_{2}{ }^{*} A_{2} A_{2}{ }^{*} L_{2}\right)\right) E_{w}\left(\Gamma_{1}^{\prime}\right)\right| L_{1}^{\prime}\right\rangle<\infty
\end{aligned}
$$

The same holds true for the other expression on the right-hand side of (3.44), which shows that $\boldsymbol{x} \mapsto\left|\left\langle L_{1}^{\prime}\left|\pi_{w}\left(\alpha_{\boldsymbol{x}}\left(L_{2}{ }^{*} A_{2}\right) A_{1}{ }^{*} L_{1}\right) U_{w}(\boldsymbol{x})\right| L_{2}^{\prime}\right\rangle\right|$ is an integrable function, too. Altogether, we have thus established the Cluster Property for particle weights.

Remark. Note, that the above result is independent of the differentiability properties of a particle weight (item (iii) in both Definition 3.14 and Theorem 3.15), since these did not enter into its proof.

At this point a brief comment on the notation chosen seems appropriate (cf. [19]). We deliberately utilize the typographical token $|$.$\rangle introduced by Dirac [23, § 23] for$ 
ket vectors describing improper momentum eigenstates $|\boldsymbol{p}\rangle, \boldsymbol{p} \in \mathbb{R}^{s}$. These act as distributions on the space of momentum wave functions with values in the physical Hilbert space $\mathcal{H}$, thereby presupposing a superposition principle to hold without limitations. This assumption collapses in an infraparticle situation as described in the Introduction. In contrast to this, the pure particle weights, that will shortly have their appearance in connection with elementary physical systems, are seen to be associated with sharp momentum and yet capable of describing infraparticles. Here the operators $L \in \mathfrak{L}$ take on the role of the previously mentioned momentum space wave functions in that they localize the particle weight in order to produce a normalizable vector $|L\rangle$ in the pertaining Hilbert space $\mathcal{H}_{w}$. This in turn substantiates the terminology introduced in Definition 2.4. As they describe elementary physical systems, pure particle weights should give rise to irreducible representations of the quasi-local algebra, thus motivating the subsequent definition. It is supplemented by a certain specific regularity condition of technical importance, which we anticipate to hold in physically relevant situations, and by a notion of boundedness which is in particular shared by the positive asymptotic functionals $\sigma$, as shown in Lemma 3.20.

Definition 3.19. A particle weight is said to be

(a) pure, if the corresponding representation $\left(\pi_{w}, \mathcal{H}_{w}\right)$ is irreducible;

(b) regular, if for any $L \in \mathfrak{L}$ the following implication is valid:

$$
\left\langle L^{*} L \mid L^{*} L\right\rangle=0 \quad \Longrightarrow \quad\langle L \mid L\rangle=0
$$

(c) $\Delta$-bounded, if to any bounded Borel subset $\Delta^{\prime}$ of $\mathbb{R}^{s+1}$ there exists another such set $\bar{\Delta} \supseteq \Delta+\Delta^{\prime}$, such that the GNS-representation $\left(\pi_{w}, \mathcal{H}_{w}\right)$ of the particle weight and the defining representation are connected by the inequality

$$
\left\|E_{w}\left(\Delta^{\prime}\right) \pi_{w}(A) E_{w}\left(\Delta^{\prime}\right)\right\| \leqslant c \cdot\|E(\bar{\Delta}) A E(\bar{\Delta})\|
$$

for any $A \in \mathfrak{A}$ with a suitable positive constant $c$ (independent of the Borel sets). Evidently, $\Delta$ ought to be a bounded Borel set as well.

Lemma 3.20. Any positive asymptotic functional $\sigma \in \mathfrak{C}_{\Delta}{ }^{*+}$, constructed according to Theorem 3.10 under the assumptions of Proposition 3.11, gives rise to a $\Delta$-bounded particle weight $\langle. \mid \cdot\rangle_{\sigma}$.

Proof. Let $\left(\pi_{\sigma}, \mathcal{H}_{\sigma}\right)$ denote the GNS-representation of the particle weight $\sigma$ with associated spectral measure $E_{\sigma}($.$) for the generator P_{\sigma}=\left(P_{\sigma}^{\mu}\right)$ of the intrinsic space-time translations. For the time being, suppose that $\Delta^{\prime}$ is an open bounded Borel set in $\mathbb{R}^{s+1}$. Let furthermore $L$ be an arbitrary element of $\mathfrak{L}$ and $A \in \mathfrak{A}$. We are interested in an estimate of the term $\left\langle L\left|E_{\sigma}\left(\Delta^{\prime}\right) \pi_{\sigma}(A) E_{\sigma}\left(\Delta^{\prime}\right)\right| L\right\rangle_{\sigma}$. Note, that the spectral measure is regular, so that $E_{\sigma}\left(\Delta^{\prime}\right)$ is the strong limit of the net $\left\{E_{\sigma}(\Gamma): \Gamma \subset \Delta^{\prime}\right.$ compact $\}$. As $\Delta^{\prime}$ is assumed to be open, there exists for each compact subset $\Gamma$ of $\Delta^{\prime}$ an infinitely often differentiable function $\tilde{g}_{\Gamma}$ with supp $\tilde{g}_{\Gamma} \subset \Delta^{\prime}$ that fits between the corresponding characteristic functions [40, Satz 7.7]: $\chi_{\Gamma} \leqslant \tilde{g}_{\Gamma} \leqslant \chi_{\Delta^{\prime}}$. Thus the respective operators are subject to the relation

$$
0 \leqslant\left(E_{\sigma}\left(\Delta^{\prime}\right)-\tilde{g}_{\Gamma}\left(P_{\sigma}\right)\right)^{2} \leqslant\left(E_{\sigma}\left(\Delta^{\prime}\right)-E_{\sigma}(\Gamma)\right)^{2},
$$


from which we infer that for arbitrary $L^{\prime} \in \mathfrak{L}$

$$
0 \leqslant \|\left(E_{\sigma}\left(\Delta^{\prime}\right)-\tilde{g}_{\Gamma}\left(P_{\sigma}\right)\right)\left|L^{\prime}\right\rangle\left\|^{2} \leqslant\right\|\left(E_{\sigma}\left(\Delta^{\prime}\right)-E_{\sigma}(\Gamma)\right)\left|L^{\prime}\right\rangle \|^{2} \underset{\Gamma / \Delta^{\prime}}{\longrightarrow} 0 .
$$

By density of all the vectors $\left|L^{\prime}\right\rangle$ in $\mathcal{H}_{\sigma}$, it is thereby established that

$$
E_{\sigma}\left(\Delta^{\prime}\right)=\text { strong }-\lim _{\Gamma \nearrow \Delta^{\prime}} \tilde{g}_{\Gamma}\left(P_{\sigma}\right),
$$

which implies for the scalar product in to be considered here

$$
\left\langle L\left|E_{\sigma}\left(\Delta^{\prime}\right) \pi_{\sigma}(A) E_{\sigma}\left(\Delta^{\prime}\right)\right| L\right\rangle_{\sigma}=\lim _{\Gamma \nearrow \Delta^{\prime}}\left\langle L\left|\tilde{g}_{\Gamma}\left(P_{\sigma}\right) \pi_{\sigma}(A) \tilde{g}_{\Gamma}\left(P_{\sigma}\right)\right| L\right\rangle .
$$

Since $\tilde{g}_{\Gamma}$ is the Fourier transform of a rapidly decreasing function $g_{\Gamma}$, which therefore belongs to the space $L^{1}\left(\mathbb{R}^{s+1}, d^{s+1} x\right)$, Lemma 3.16 can be applied to yield for the righthand side of (3.49)

$$
\begin{aligned}
\left\langle L\left|\tilde{g}_{\Gamma}\left(P_{\sigma}\right) \pi_{\sigma}(A) \tilde{g}_{\Gamma}\left(P_{\sigma}\right)\right| L\right\rangle=(2 \pi)^{-(s+1)}\left\langle\alpha_{g_{\Gamma}}(L)\left|\pi_{\sigma}(A)\right| \alpha_{g_{\Gamma}}(L)\right\rangle_{\sigma} \\
=(2 \pi)^{-(s+1)} \sigma\left(\alpha_{g_{\Gamma}}(L)^{*} A \alpha_{g_{\Gamma}}(L)\right),
\end{aligned}
$$

where, following the remark pertaining to Proposition 3.11, the ultimate expression is based on the fact that $\alpha_{g_{\Gamma}}(L)^{*} A \alpha_{g_{\Gamma}}(L) \in \mathfrak{A}_{\mathfrak{C}}$ as a consequence of Lemmas 2.18 and 2.12 in connection with Corollary 2.13. The approximating functionals $\rho_{h, t}$ for $\sigma$ in the form (3.23) with a non-negative function $h \in L^{\infty}\left(\mathbb{R}^{s}, d^{s} x\right)$ allow, through an application of [11, Proposition 2.3.11], for the following estimate of their integrand:

$$
\begin{aligned}
& \left|h\left(\tau^{-1} \boldsymbol{x}\right) \omega\left(U(\tau) E(\Delta) \alpha_{\boldsymbol{x}}\left(\alpha_{g_{\Gamma}}(L)^{*} A \alpha_{g_{\Gamma}}(L)\right) E(\Delta) U(\tau)^{*}\right)\right| \\
& =h\left(\tau^{-1} \boldsymbol{x}\right)\left|\omega\left(U(\tau) E(\Delta) \alpha_{\boldsymbol{x}}\left(\alpha_{g_{\Gamma}}(L)^{*}\right) E(\bar{\Delta}) \alpha_{\boldsymbol{x}}(A) E(\bar{\Delta}) \alpha_{\boldsymbol{x}}\left(\alpha_{g_{\Gamma}}(L)\right) E(\Delta) U(\tau)^{*}\right)\right| \\
& \quad \leqslant\|E(\bar{\Delta}) A E(\bar{\Delta})\| h\left(\tau^{-1} \boldsymbol{x}\right) \omega\left(U(\tau) E(\Delta) \alpha_{\boldsymbol{x}}\left(\alpha_{g_{\Gamma}}(L)^{*} \alpha_{g_{\Gamma}}(L)\right) E(\Delta) U(\tau)^{*}\right) .
\end{aligned}
$$

Here the spectral projections $E(\bar{\Delta})$ pertaining to the Borel set $\bar{\Delta}=\Delta+\Delta^{\prime}$, which is both bounded and open, could be introduced, since, according to Lemma 2.18, the energy-momentum transfer of $\alpha_{g_{\Gamma}}(L)$ is contained in $\Delta^{\prime}$ by construction. An immediate consequence of the above relation is

$$
\left|\rho_{h, t}\left(\alpha_{g_{\Gamma}}(L)^{*} A \alpha_{g_{\Gamma}}(L)\right)\right| \leqslant\|E(\bar{\Delta}) A E(\bar{\Delta})\| \rho_{h, t}\left(\alpha_{g_{\Gamma}}(L)^{*} \alpha_{g_{\Gamma}}(L)\right),
$$

which extends to the limit functional $\sigma$ :

$$
\left|\sigma\left(\alpha_{g_{\Gamma}}(L)^{*} A \alpha_{g_{\Gamma}}(L)\right)\right| \leqslant\|E(\bar{\Delta}) A E(\bar{\Delta})\| \sigma\left(\alpha_{g_{\Gamma}}(L)^{*} \alpha_{g_{\Gamma}}(L)\right) .
$$

Insertion of this result into (3.50) yields

$$
\left|\left\langle L\left|\tilde{g}_{\Gamma}\left(P_{\sigma}\right) \pi_{\sigma}(A) \tilde{g}_{\Gamma}\left(P_{\sigma}\right)\right| L\right\rangle\right| \leqslant\|E(\bar{\Delta}) A E(\bar{\Delta})\|\left\langle L\left|\tilde{g}_{\Gamma}\left(P_{\sigma}\right)^{2}\right| L\right\rangle
$$

and in the limit $\Gamma \nearrow \Delta^{\prime}$, in compliance with (3.49),

$$
\left|\left\langle L\left|E_{\sigma}\left(\Delta^{\prime}\right) \pi_{\sigma}(A) E_{\sigma}\left(\Delta^{\prime}\right)\right| L\right\rangle_{\sigma}\right| \leqslant\|E(\bar{\Delta}) A E(\bar{\Delta})\|\left\langle L\left|E_{\sigma}\left(\Delta^{\prime}\right)\right| L\right\rangle_{\sigma} \leqslant\|E(\bar{\Delta}) A E(\bar{\Delta})\|\langle L \mid L\rangle_{\sigma} .
$$


Passing to the supremum with respect to all $L \in \mathfrak{L}$ such that $\||L\rangle_{\sigma} \| \leqslant 1$ (these constitute a dense subset of the unit ball in $\mathcal{H}_{\sigma}$ ), we get through an application of [56, Satz 4.4]

$$
\left\|E_{\sigma}\left(\Delta^{\prime}\right) \pi_{\sigma}(A) E_{\sigma}\left(\Delta^{\prime}\right)\right\| \leqslant 2 \cdot\|E(\bar{\Delta}) A E(\bar{\Delta})\| .
$$

This establishes the defining condition (3.46) for $\Delta$-boundedness with $c=2$ in the case of an open bounded Borel set $\Delta^{\prime}$. But this is not an essential restriction, since an arbitrary bounded Borel set $\Delta^{\prime}$ is contained in the open set $\Delta_{\eta}^{\prime}, \eta>0$, consisting of all those points $p \in \mathbb{R}^{s+1}$ for which $\inf _{p^{\prime} \in \Delta^{\prime}}\left|p-p^{\prime}\right|<\eta$. Since $\Delta_{\eta}^{\prime}$ is likewise a bounded Borel set, we get

$$
\left\|E_{\sigma}\left(\Delta^{\prime}\right) \pi_{\sigma}(A) E_{\sigma}\left(\Delta^{\prime}\right)\right\| \leqslant\left\|E_{\sigma}\left(\Delta_{\eta}^{\prime}\right) \pi_{\sigma}(A) E_{\sigma}\left(\Delta_{\eta}^{\prime}\right)\right\| \leqslant 2 \cdot\left\|E\left(\bar{\Delta}_{\eta}\right) A E\left(\bar{\Delta}_{\eta}\right)\right\|
$$

as an immediate consequence of (3.54), where $\bar{\Delta}_{\eta} \doteq \Delta+\Delta_{\eta}^{\prime}$. This covers the general case and thereby proves $\Delta$-boundedness for the asymptotic functionals $\sigma \in \mathfrak{C}_{\Delta}{ }^{*+}$. 


\title{
Chapter 4
}

\section{Disintegration of Particle Weights}

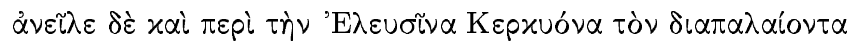

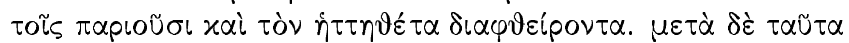

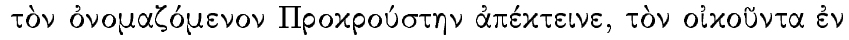

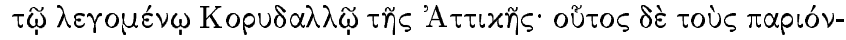

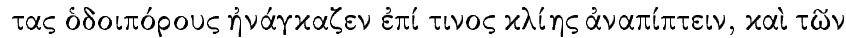

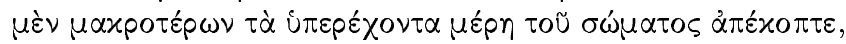

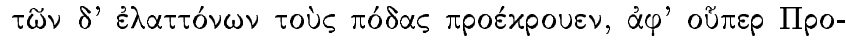

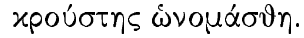

$\Delta \mathrm{IO} \Delta \mathrm{OPO}$ BIB $\Lambda \mathrm{IO} \Theta H K E$ I $\Sigma$ TOPIKH IV, $59(5) *$

In Section III of their treatment of collision cross sections for massive theories within the framework of local quantum physics, Araki and Haag got to the following asymptotic relation which holds true for the counters $C$ they had selected, for arbitrary vectors $\Phi$ and certain specific vectors $\Psi$ representing outgoing particle configurations [3, Theorem 4]:

$$
\lim _{t \rightarrow \infty}\left\langle\Phi\left|t^{3} C(h, t)\right| \Psi\right\rangle=\sum_{i, j}^{\prime} \int d^{3} p \Gamma_{i j}(\boldsymbol{p})\left\langle\Phi\left|a_{j}^{\text {†out }}(\boldsymbol{p}) a_{i}^{\text {out }}(\boldsymbol{p})\right| \Psi\right\rangle h\left(\boldsymbol{v}_{i}\right),
$$

where

$$
\begin{aligned}
\Gamma_{i j}(\boldsymbol{p}) & \doteq 8 \pi^{3}\langle\boldsymbol{p} j|C(0)| \boldsymbol{p} i\rangle \\
\boldsymbol{v}_{i} & \doteq\left(\boldsymbol{p}^{2}+m_{i}^{2}\right)^{-1 / 2} \boldsymbol{p}
\end{aligned}
$$

The indices $i$ and $j$ in the above formula denote the particle types including spin, and summation runs over pairs of particles with equal mass: $m_{i}=m_{j}$. The structure of the right-hand side of this equation is based on the a priori knowledge of the particle content of the theory they considered. Comparing this result with the concepts developed in the preceding chapter (cf. Theorem 3.10), one has an asymptotic functional $\sigma_{h}^{(+)}$standing on the left-hand side of equation (4.1) that is decomposed with respect to momentum eigenstates $|\boldsymbol{p} i\rangle$, hidden in the definition of $\Gamma_{i j}$. If we accept such an

${ }^{*}$ A german translation can be found on page 120 . 
interpretation of this theorem of Araki and Haag, it is possible to re-write it in the form

$$
\boldsymbol{\sigma}_{h}^{(+)}(C)=\sum_{i, j}^{\prime} \int d \mu_{i, j}(\boldsymbol{p})\langle\boldsymbol{p} j|C(0)| \boldsymbol{p} i\rangle,
$$

where all expressions occurring in (4.1) apart from $\Gamma_{i j}$ are absorbed into the measures $\mu_{i, j}$. This presents the asymptotic functional as a mixture of linear forms on $\mathfrak{C}$ (an algebra which is part of that selected in [3]) defined by Dirac kets representing improper momentum eigenstates; thus we happen to meet exactly those constructs that we already hinted at in the remarks concerning our notation that led to Definition 3.19. The aim of the present chapter is to establish a corresponding formula in the general setting, i. e. without any previous knowledge of the particle content.

As indicated by (4.1), representations resulting from the construction of asymptotic functionals as expounded in Chapter 3 will be highly reducible, whereas elementary physical systems are expected to be connected with pure particle weights, giving rise to irreducible representations of the quasi-local $C^{*}$-algebra $\mathfrak{A}$. In view of the preceding paragraph the obvious problem to be tackled now is to develop a theory for the decomposition or rather disintegration of generic particle weights into pure ones. Two approaches to this problem will be presented in this work:

(A) Decomposition of the GNS-representation pertaining to a particle weight into a direct integral of representations (spatial disintegration):

$$
\left(\pi_{w}, \mathcal{H}_{w}\right) \simeq \int_{\mathrm{X}}^{\oplus} d v(\xi)\left(\pi_{\xi}, \mathcal{H}_{\xi}\right)
$$

(B) Barycentric decomposition of a given particle weight with respect to a base $\mathrm{B}_{W}$ of the positive cone $W$ of all particle weights in the space of sesquilinear forms on $\mathfrak{L}$ (Choquet theory):

$$
\langle\cdot \mid \cdot\rangle=\int_{\mathrm{B}_{W}} d v(\zeta)\langle\cdot \mid \cdot\rangle_{\zeta}
$$

Although the technical problems to come to grips with in these two constructions are quite different, we anticipate equivalence of their results: the separability assumptions essential in the first one are substituted by compactness conditions in the second. So evidently both of them require certain restrictions in the number of degrees of freedom, which seem to be complementary in one way or another. While the partial results achieved so far in connection with the barycentric decomposition will be discussed in Chapter 6, the spatial disintegration of the GNS-representation of a particle weight is the subject we will elaborate on first.

\subsection{Separable Reformulation of Local Quantum Physics and its Associated Algebra of Detectors}

The theory of spatial disintegration of representations $(\bar{\pi}, \overline{\mathcal{H}})$ of a $C^{*}$-algebra $\overline{\mathfrak{A}}$ is a common theme of the pertinent textbooks (cf. $[24,25,54,48,11]$ ), an indispensable 
presupposition being that of separability of the algebra $\overline{\mathfrak{A}}$ as well as of the Hilbert space $\overline{\mathcal{H}}$ in their respective uniform topologies. Note, that in this way the statements of [11, Section 4.4] are incorrect (cf. also [12, Corrigenda]). These separability assumptions are too restrictive to be encountered in physically reasonable theories from the outset, so first of all a countable version of the fundamental assumptions of local quantum field theory in terms of the net $\mathcal{O} \mapsto \mathfrak{A}(\mathcal{O})$ and of the symmetry group $\mathrm{P}_{+}^{\uparrow}$ has to be formulated before one can benefit from the extensive theory made available in the literature. This construction will be accomplished in a sequence of steps:

(1) With respect to its initial topology, the Poincaré group $P_{+}^{\uparrow}$ contains a numerable dense subgroup that we signify by $\mathrm{P}^{c}$. It is itself the semi-direct product of countable dense subgroups of Lorentz transformations $\mathrm{L}^{c}$ in $\mathrm{L}_{+}^{\uparrow}$ and of space-time translations $\mathrm{T}^{c}$ in $\mathbb{R}^{s+1}: \mathrm{P}^{c}=\mathrm{L}^{c} \ltimes \mathrm{T}^{c}$.

(2) Consider the standard diamonds with rational radii, centred around the origin. Subjecting these regions to all of the transformations in $\mathrm{P}^{c}$ yields a countable family $\mathcal{R}^{c}$ of open bounded regions, which is invariant with respect to the selected Poincaré transformations and constitutes a covering of $\mathbb{R}^{s+1}$. Note, that arbitrarily small regions belong to $\mathcal{R}^{c}$ in the sense, that any region in Minkowski space contains an element of this numerable collection as a subset.

(3) As shown in Appendix B, any unital $C^{*}$-algebra of operators on a separable Hilbert space $\mathcal{H}$ contains a strongly dense (i. e. dense with respect to the strong-operator topology), norm-separable $C^{*}$-subalgebra, that includes the identity. Applied to the local $C^{*}$-algebras $\mathfrak{A}(\mathcal{O})$ of the defining positive-energy representation, this result has the consequence that to each open bounded region $\mathcal{O}$ in Minkowski space one can associate a norm-separable, unital $C^{*}$-algebra $\mathfrak{A}_{\bullet}(\mathcal{O})$, that lies strongly dense in $\mathfrak{A}(\mathcal{O})$. This means, that the algebra $\mathfrak{A}_{\bullet}(\mathcal{O})$ in turn contains a countable *-subalgebra $\mathfrak{A}^{c}(\mathcal{O})$ over the field $\mathbb{Q}+i \mathbb{Q}$, which is uniformly dense in $\mathfrak{A}_{\bullet}(\mathcal{O})$, strongly dense in $\mathfrak{A}(\mathcal{O})$ and can likewise be chosen to comprise the unit.

Let $\mathcal{O}_{k}, k \in \mathbb{N}$, be a denumeration of the countable family $\mathcal{R}^{c}$ of open bounded regions in Minkowski space constructed above. We define $\mathfrak{A}^{\bullet}\left(\mathcal{O}_{k}\right)$ as the $C^{*}$-algebra (over $\mathbb{C}$ ) which is generated by the union of all $\alpha_{(\Lambda, x)}\left(\mathfrak{A}^{c}\left(\mathcal{O}_{i}\right)\right)$, where $(\Lambda, x) \in \mathrm{P}^{c}$ and $\mathcal{O}_{i} \in \mathcal{R}^{c}$ run through all combinations for which $\Lambda \mathcal{O}_{i}+x \subseteq \mathcal{O}_{k}$. By construction this algebra is norm-separable and satisfies

$$
\mathfrak{A}^{c}\left(\mathcal{O}_{k}\right) \subseteq \mathfrak{A}^{\bullet}\left(\mathcal{O}_{k}\right) \subseteq \mathfrak{A}\left(\mathcal{O}_{k}\right),
$$

so that $\mathfrak{A}^{\bullet}\left(\mathcal{O}_{k}\right)$ turns out to be strongly dense in $\mathfrak{A}\left(\mathcal{O}_{k}\right)$.

The net of local $C^{*}$-algebras $\left\{\mathfrak{A}^{\bullet}\left(\mathcal{O}_{k}\right): k \in \mathbb{N}\right\}$ fulfills the conditions of isotony, locality and covariance with respect to $\mathcal{R}^{c}$ and $\mathrm{P}^{c}$. Isotony is an immediate consequence of the construction whereas locality follows from (4.2a) in connection with locality of the defining net $\mathcal{O} \mapsto \mathfrak{A}(\mathcal{O})$. To establish covariance one has to observe that, given any $(\Lambda, x) \in \mathrm{P}^{c}$, the algebra $\alpha_{(\Lambda, x)}\left(\mathfrak{A}^{\bullet}\left(\mathcal{O}_{k}\right)\right)$ is generated by all $\alpha_{(\Lambda, x)}\left(\alpha_{\left(\Lambda^{\prime}, x^{\prime}\right)}\left(\mathfrak{A}^{c}\left(\mathcal{O}_{i}\right)\right)\right)$, where $\left(\Lambda^{\prime}, x^{\prime}\right) \in \mathrm{P}^{c}$ and $\mathcal{O}_{i} \in \mathcal{R}^{c}$ run through those combinations which satisfy the relation $\Lambda^{\prime} \mathcal{O}_{i}+x^{\prime} \subseteq \mathcal{O}_{k}$. This can equivalently be expressed by saying that the algebra in question is generated by all $\alpha_{\left(\Lambda^{\prime \prime}, x^{\prime \prime}\right)}\left(\mathfrak{A}^{c}\left(\mathcal{O}_{i}\right)\right)$, for which $\left(\Lambda^{\prime \prime}, x^{\prime \prime}\right) \in \mathrm{P}^{c}$ and $\mathcal{O}_{i} \in \mathcal{R}^{c}$ have the property $\Lambda^{\prime \prime} \mathcal{O}_{i}+x^{\prime \prime} \subseteq \Lambda \mathcal{O}_{k}+x$. In this formulation $\alpha_{(\Lambda, x)}\left(\mathfrak{A}^{\bullet}\left(\mathcal{O}_{k}\right)\right)$ turns out to be equal to the algebra $\mathfrak{A}^{\bullet}\left(\Lambda \mathcal{O}_{k}+x\right)$. The somewhat intricate construction of $\mathfrak{A}^{\bullet}\left(\mathcal{O}_{k}\right)$ is 
necessitated by the requisite to have the standard properties of a net of local algebras at our disposition.

By construction, the countable ${ }^{*}$-algebra $\mathfrak{A}^{c}$ over $\mathbb{Q}+i \mathbb{Q}$, which is generated by the union of all the algebras $\mathfrak{A}^{c}\left(\mathcal{O}_{k}\right), \mathcal{O}_{k} \in \mathcal{R}^{c}$, and thus invariant under transformations from $\mathrm{P}^{c}$, lies uniformly dense in the $C^{*}$-inductive limit $\mathfrak{A}^{\bullet}$ of the net $\mathcal{O}_{k} \mapsto \mathfrak{A}^{\bullet}\left(\mathcal{O}_{k}\right)$, and is, on account of (4.2a), even strongly dense in the quasi-local algebra $\mathfrak{A}$ itself. We thus have the inclusions

$$
\mathfrak{A}^{c} \subseteq \mathfrak{A}^{\bullet} \subseteq \mathfrak{A},
$$

with a norm-separable $C^{*}$-algebra $\mathfrak{A}^{\bullet}$, which lies strongly dense in $\mathfrak{A}$ and contains $\mathfrak{A}^{c}$ as a numerable uniformly dense subalgebra (over $\mathbb{Q}+i \mathbb{Q}$ ).

Into this restricted setting of Local Quantum Physics defined above, we now introduce countable counterparts of the left ideal of localizing operators $\mathfrak{L}$, of the algebra of detectors $\mathfrak{C}$ and, most important of all, of the subspace $\mathfrak{L}_{0} \subseteq \mathfrak{L}$ of almost local vacuum annihilation operators.

First of all note, that it is possible to select a numerable subspace over $\mathbb{Q}+i \mathbb{Q}$ in $\mathfrak{L}_{0}$, which consists of almost local vacuum annihilation operators with energy-momentum transfer in arbitrarily small regions. E. g. let $\left\{\Gamma_{n}\right\}_{n \in \mathbb{N}}$ be a countable cover of $\complement \bar{V}_{+}$, constituted by compact and convex subsets of the complement of the forward light cone, with the additional property that any bounded region in $C \bar{V}_{+}$contains one of these compacta. Let, for instance, $\left\{p_{i}\right\}_{i \in \mathbb{N}}$ be a dense sequence in $C \bar{V}_{+}$and associate to each $p_{i}$ the compact balls of rational radius $r \in \mathbb{Q}$ that satisfy $\overline{\mathcal{B}_{r}\left(p_{i}\right)} \subseteq \complement \bar{V}_{+}$in addition. The Lorentz group $L_{+}^{\uparrow}$, being locally compact, can be covered by a countable family of arbitrarily small compact sets $\left\{\Theta_{m}\right\}_{m \in \mathbb{N}}$ as well. Now, the spaces $\mathcal{D}_{\Gamma_{n}}$ and $\mathcal{D}_{\Theta_{m}}$ of test functions with support in $\Gamma_{n}$ or else $\Theta_{m}$ (cf. [40, § 12]) are separable as subspaces of the respective Banach spaces $L^{p}\left(\mathbb{R}^{s+1}, d^{s+1} x\right)$ and $L^{p}\left(\mathbb{R}^{d_{\mathrm{L}}}, d^{d_{\mathrm{L}}} t\right)\left(d_{\mathrm{L}} \doteq\right.$ $2^{-1} s(s+1)$ is the dimension of $\left.\mathrm{L}_{+}^{\uparrow}\right)$, which in turn are separable due to an application of [39, Theorem IV.(13.20)] using elements of the numerable set of simple functions with rational values on intervals with rational end points. Thus there exist dense sequences $\tilde{g}_{n}^{l}$ and $h_{m}^{k}$ in the spaces $\mathcal{D}_{\Gamma_{n}}$ and $\mathcal{D}_{\Theta_{m}}$, respectively. Consider the countable family of operators in $\mathfrak{L}_{0}$, which are defined through

$$
\alpha_{h_{m}^{k} \otimes g_{n}^{l}}\left(A_{j}\right) \doteq \int_{\mathrm{P}_{+}^{\dagger}} d \mu(\Lambda, x) h_{m}^{k}(\Lambda) g_{n}^{l}(x) \alpha_{(\Lambda, x)}\left(A_{j}\right),
$$

for any $A_{j} \in \mathfrak{A}^{c}$ in the uniform topology of $\mathfrak{A}$, and supplement this selection by all orders of partial derivatives with respect to the canonical coordinates around $(\mathbf{1}, 0)$ (cf. Appendix A):

$$
\delta^{l_{M}}\left(\alpha_{h_{m}^{k} \otimes g_{n}^{l}}\left(A_{j}\right)\right)=\delta^{i_{M}} \cdots \delta^{i_{1}}\left(\alpha_{h_{m}^{k} \otimes g_{n}^{l}}\left(A_{j}\right)\right) \in \mathfrak{L}_{0}
$$

for any $M$-tuple $i_{M}=\left(i_{1}, \ldots, i_{M}\right)$ with integer entries from the set $\left\{1, \ldots, d_{\mathrm{P}}\right\}$, where $d_{\mathrm{P}}=d_{\mathrm{L}}+(s+1)$. Upon application of all transformations from $\mathrm{P}^{c}$ to these constructs, we get a sequence of vacuum annihilation operators, comprising elements with energymomentum transfer in arbitrarily small regions, which generates a countable subspace $\mathfrak{L}_{0}^{c}$ over the field $\mathbb{Q}+i \mathbb{Q}$ in $\mathfrak{L}_{0}$, invariant under transformations from $\mathrm{P}^{c}$ and under arbitrary partial derivations. When this construct is to be used in connection with a 
given particle weight $\langle. \mid$.$\rangle that is non-negative by definition, it does not cause any$ problems to supplement the set of operators defined in (4.3) by a countable number of other elements from $\mathfrak{L}_{0}$, on which the particle weight attains non-vanishing values. In this way the imminent restriction of $\langle. \mid$.$\rangle to a subset of \mathfrak{L}$ can be protected from getting trivial.

The above selection of vacuum annihilation operators does not yet meet the requirements for the disintegration. For it to be feasible we compactly regularize these operators: Take a countable set of compactly supported test functions $F$ on $\mathrm{P}_{+}^{\uparrow}$ with a support $\mathrm{S}_{F} \doteq \operatorname{supp} F$ which contains the unit $(\mathbf{1}, 0)$ of $\mathrm{P}_{+}^{\uparrow}$. Then all the Bochner integrals

$$
\alpha_{F}\left(L_{0}\right)=\int_{\mathrm{S}_{F}} d \mu(\Lambda, x) F(\Lambda, x) \alpha_{(\Lambda, x)}\left(L_{0}\right), \quad L_{0} \in \mathfrak{L}_{0}^{c},
$$

are elements of the $C^{*}$-algebra $\mathfrak{A}^{\bullet}$ and of $\mathfrak{L}_{0}$ according to Lemma 2.17 with energymomentum transfer contained in $\bigcup_{(\Lambda, x) \in \mathrm{S}_{F}} \Lambda \Gamma$ given $L_{0} \in \mathfrak{L}_{0}(\Gamma)$ (cf. the proof of the quoted Lemma). The specific property of operators of type (4.4) in contrast to those from $\mathfrak{L}_{0}^{c}$ is, that their differentiability with respect to the Poincare group can be expressed in terms of derivatives of the infinitely differentiable test function $F \in \mathcal{D}_{\mathrm{S}_{F}}$, a feature that will be of great significance later on. By choosing the support of the functions $F$ small enough, one can impose an energy-momentum transfer in arbitrarily small regions on the operators $\alpha_{F}\left(L_{0}\right)$ as was the case for the elements of $\mathfrak{L}_{0}^{c}$ itself. Furthermore, a particle weight that did not vanish on the set $\mathfrak{L}_{0}^{c}$ is also non-zero when restricted to all of the operators $\alpha_{F}\left(L_{0}\right)$ constructed in (4.4). This fact is easily established with relation (3.39b) of Lemma 3.16 and the continuity of the particle weight under Poincaré transformations in mind. The numerable set of vacuum annihilation operators that consists of those explicitly presented in (4.4) together with all their partial derivations of arbitrary order (that share this specific style of construction) will be denoted $\overline{\mathfrak{L}_{0}^{c}}$ in the sequel. It might happen that two of these elements of $\mathfrak{L}_{0}$ are connected by a Poincaré transformation not yet included in $\mathrm{P}^{c}$. For technical reasons, which are motivated by the exigencies for the proof of the central Theorem 4.4 of this chapter, we supplement $\mathrm{P}^{c}$ by all of the (countably many) transformations arising in this way and consider henceforth the countable subgroup $\overline{\mathrm{P}}^{c}=\overline{\mathrm{L}}^{c} \ltimes \overline{\mathrm{T}}^{c} \subseteq \mathrm{P}_{+}^{\uparrow}$ generated by them. The set $\overline{\mathfrak{L}_{0}^{c}}$ is then invariant under the operation of taking derivatives as well as under all transformations from the numerable dense subgroup $\overline{\mathrm{P}}^{c}$.

Here is a list of the countable substitutes for the algebraic concepts used thus far:

(I) We have defined an isotonous, local and $\mathrm{P}^{c}$-covariant net $\mathcal{O}_{k} \mapsto \mathfrak{A}^{\bullet}\left(\mathcal{O}_{k}\right), \mathcal{O}_{k} \in \mathcal{R}^{c}$, which has $\mathfrak{A}^{\bullet}$ as $C^{*}$-inductive limit. This is a norm-separable $C^{*}$-algebra (over the field $\mathbb{C}$ ) with unit $\mathbf{1}$, containing $\mathfrak{A}^{c}$, which is generated by the countable local algebras $\mathfrak{A}^{c}\left(\mathcal{O}_{k}\right) \subseteq \mathfrak{A}^{\bullet}\left(\mathcal{O}_{k}\right)$, as a likewise unital, numerable, uniformly dense $*$-subalgebra over $\mathbb{Q}+i \mathbb{Q} \cdot \mathfrak{A}^{\bullet}$ itself lies strongly dense in the quasi-local algebra $\mathfrak{A}$ and, due to uniform continuity of the mappings $(\Lambda, x) \mapsto \alpha_{(\Lambda, x)}(A), A \in \mathfrak{A}$, it is invariant with respect to the whole Poincaré group. In contrast to this, note, that the invariance property for $\mathfrak{A}^{c}$ is restricted to $\mathrm{P}^{c}$.

(II) $\overline{\mathfrak{L}_{0}^{c}} \subseteq \mathfrak{L}_{0} \cap \mathfrak{A}^{\bullet}$ is a countable set of vacuum annihilation operators of the special construction (4.4), which is invariant under transformations from $\overline{\mathrm{P}}^{c}$ and under the 
operation of taking partial derivations. Depending on a given particle weight, it can be chosen in such a way, that the particle weight restricted to $\overline{\mathfrak{L}_{0}^{c}}$ remains non-trivial.

(III) The image of $\overline{\mathfrak{L}_{0}^{c}}$ under all Poincaré transformations is denoted $\overline{\mathfrak{L}_{0}}$ :

$$
\overline{\mathfrak{L}_{0}} \doteq\left\{\alpha_{(\Lambda, x)}\left(L_{0}\right): L_{0} \in \overline{\mathfrak{L}_{0}^{c}},(\Lambda, x) \in \mathrm{P}_{+}^{\uparrow}\right\}
$$

(IV) $\overline{\mathfrak{A}^{c}} \subseteq \mathfrak{A}^{\bullet}$ in turn denotes the numerable, unital ${ }^{*}$-algebra over $\mathbb{Q}+i \mathbb{Q}$ which is generated by $\mathfrak{A}^{c} \cup \overline{\mathfrak{L}_{0}^{c}}$. It is thus stable with respect to $\mathrm{P}^{c}$ and uniformly dense in $\mathfrak{A}^{\bullet}$.

(V) The countable counterpart $\mathfrak{L}^{c}$ of the left ideal $\mathfrak{L}$ in $\mathfrak{A}$ is defined as the linear span with respect to the field $\mathbb{Q}+i \mathbb{Q}$ of operators of the form $L=A L_{0}$ with $A \in \overline{\mathfrak{A}^{c}}$ and $L_{0} \in \overline{\mathfrak{L}_{0}^{c}}$ :

$$
\mathfrak{L}^{c} \doteq \overline{\mathfrak{A}^{c}} \overline{\mathfrak{L}_{0}^{c}}=\operatorname{span}_{\mathbb{Q}+i \mathbb{Q}}\left\{A L_{0}: A \in \overline{\mathfrak{A}^{c}}, L_{0} \in \overline{\mathfrak{L}_{0}^{c}}\right\}
$$

This constitutes a left ideal of the algebra $\overline{\mathfrak{A}^{c}}$, likewise invariant under transformations from $\mathrm{P}^{c}$.

(VI) Finally, one can introduce the countable ${ }^{*}$-subalgebra $\mathfrak{C}^{c} \subseteq \mathfrak{C}$ via

$$
\mathfrak{C}^{c} \doteq \mathfrak{L}^{c *} \mathfrak{L}^{c}=\operatorname{span}_{\mathbb{Q}+i \mathbb{Q}}\left\{L_{1}^{*} L_{2}: L_{1}, L_{2} \in \mathfrak{L}^{c}\right\}
$$

\subsection{Restricted $\mathfrak{K}_{0}^{c}$-Particle Weights}

The subsequent developments in this chapter have to be founded on a mitigated version for the concept of particle weights as it was introduced in Definition 3.14. The reason is that the sesquilinear forms occurring in the decomposition theory of Section 4.3 do not share all the desired properties. Therefore we insert the present section which deals with the necessary restrictions that have to be imposed on the concepts of Chapter 3. The essential cuts are indicated by the work previously accomplished.

Definition 4.1 (Restricted $\mathfrak{K}_{0}^{c}$-Particle Weights). Suppose that we are given a sextuple $\left(\underline{\pi}, \underline{\mathcal{H}}, \underline{\mathfrak{A}}, \underline{\alpha}, \underline{\mathrm{P}}^{c}, \mathfrak{K}_{0}^{c}\right)$ with entries of the following sense:

- $\mathfrak{A}$ is a norm separable $C^{*}$-subalgebra of the quasi-local algebra $\mathfrak{A}$, which arises as the $C^{*}$-inductive limit of a countable net of local $C^{*}$-algebras.

- $\underline{\mathrm{P}}^{c}$ is a numerable dense subgroup of the Poincaré group. $\mathrm{P}_{+}^{\uparrow}$ as a whole is implemented in $\underline{A}$ by the strongly continuous group of automorphisms

$$
\left\{\underline{\alpha}_{(\Lambda, x)}:(\Lambda, x) \in \mathrm{P}_{+}^{\uparrow}\right\} \subseteq \operatorname{Aut} \underline{\mathfrak{A}} .
$$

- $\mathfrak{K}_{0}^{c}$ designates a countable set of almost local vacuum annihilation operators in $\underline{\mathfrak{A}}$, stable with respect to transformations from $\underline{\mathrm{P}}^{c}$. The image of $\mathfrak{K}_{0}^{c}$ under all Poincaré transformations is denoted $\mathfrak{K}_{0}$ :

$$
\mathfrak{K}_{0} \doteq\left\{\underline{\alpha}_{(\Lambda, x)}\left(K_{0}\right): K_{0} \in \mathfrak{K}_{0}^{c},(\Lambda, x) \in \mathrm{P}_{+}^{\uparrow}\right\} .
$$

- Together with the numerable uniformly dense *-subalgebra of $\mathfrak{A}$, which exists by construction, $\mathfrak{K}_{0}^{c}$ generates a countable ${ }^{*}$-algebra over $\mathbb{Q}+i \mathbb{Q}$, denoted $\underline{\mathfrak{A}}^{c}$ and likewise invariant under $\underline{\mathrm{P}}^{c}$. 
A countable left ideal in $\underline{\mathfrak{A}}^{c}$ is then defined by

$$
\mathfrak{K}^{c} \doteq \underline{\mathfrak{A}}^{c} \mathfrak{K}_{0}^{c}=\operatorname{span}_{\mathbb{Q}+i \mathbb{Q}}\left\{A K_{0}: A \in \underline{\mathfrak{A}}^{c}, K_{0} \in \mathfrak{K}_{0}^{c}\right\} .
$$

It is invariant under the automorphism group $\left\{\underline{\alpha}_{(\Lambda, x)}:(\Lambda, x) \in \underline{\mathrm{P}}^{c}\right\}$ as well.

- Finally, $(\underline{\pi}, \underline{\mathcal{H}})$ is a non-zero, non-degenerate representation of the $C^{*}$-algebra $\underline{\mathfrak{A}}$.

The sextuple $\left(\underline{\pi}, \underline{\mathcal{H}}, \underline{\mathfrak{A}}, \underline{\alpha}, \underline{\mathrm{P}}^{c}, \mathfrak{K}_{0}^{c}\right)$ is called a restricted $\mathfrak{K}_{0}^{c}$-particle weight, in case that it complies with the following list of features:

(i) There exists a $(\mathbb{Q}+i \mathbb{Q})$-linear mapping $\mid$. $\rangle$ from $\mathfrak{K}^{c}$ onto a dense subset $\underline{\mathcal{H}}^{c} \subseteq \underline{\mathcal{H}}$ :

$$
|.\rangle: \mathfrak{K}^{c} \rightarrow \underline{\mathcal{H}}^{c} \quad K \mapsto|K\rangle,
$$

such that the representation $\underline{\pi}$ acts on this space according to

$$
\underline{\pi}(A)|K\rangle=|A K\rangle, \quad A \in \underline{\mathfrak{A}}^{c}, \quad K \in \mathfrak{K}^{c} .
$$

(ii) The above linear mapping allows for an extension to any operator in $\mathfrak{K}_{0}$, such that (in the notation of Theorem 3.15)

$$
\left|\Xi_{K^{\prime}}(.)\right\rangle: \mathrm{P}_{+}^{\uparrow} \rightarrow \underline{\mathcal{H}} \quad(\Lambda, x) \mapsto\left|\Xi_{K^{\prime}}(\Lambda, x)\right\rangle \doteq\left|\underline{\alpha}_{(\Lambda, x)}\left(K^{\prime}\right)\right\rangle, \quad K^{\prime} \in \mathfrak{K}_{0},
$$

is a continuous mapping.

(iii) There exists a strongly continuous unitary representation $x \mapsto \underline{U}(x)$ of space-time translations $x \in \mathbb{R}^{s+1}$ with spectral measure $\Delta \mapsto \underline{E}(\Delta)$, supported by a displaced forward light cone $\bar{V}_{+}-q, q \in \bar{V}_{+}$, which implements these transformations in the representation $(\underline{\pi}, \underline{\mathcal{H}})$ via

$$
\underline{U}(x) \underline{\pi}(A) \underline{U}(x)^{*}=\underline{\pi}\left(\underline{\alpha}_{x}(A)\right), \quad A \in \underline{\mathfrak{A}}, \quad x \in \mathbb{R}^{s+1} .
$$

On the subset $\left\{\left|K^{\prime}\right\rangle: K^{\prime} \in \mathfrak{K}_{0}\right\}$ of $\underline{\mathcal{H}}$ this unitary group acts according to

$$
\underline{U}(x)\left|K^{\prime}\right\rangle=\left|\underline{\alpha}_{x}\left(K^{\prime}\right)\right\rangle, \quad K^{\prime} \in \mathfrak{K}_{0},
$$

and there holds the relation

$$
\underline{E}\left(\Delta^{\prime}\right)|K\rangle=|K\rangle, \quad K \in \mathfrak{K}\left(\Delta^{\prime}\right),
$$

where $\mathfrak{K}\left(\Delta^{\prime}\right)$ denotes the set of operators from $\mathfrak{K}^{c} \cup \mathfrak{K}_{0}$ with energy-momentum transfer in the Borel set $\Delta^{\prime} \subseteq \mathbb{R}^{s+1}$.

Through (4.9c) we have explicitly installed into the definition of restricted $\mathfrak{K}_{0}^{c}$-particle weights the result of Proposition 3.17 for generic particle weights. A spectral assumption of this kind is of great importance since it constitutes the basis for the proof of the Cluster Property of Proposition 3.18, and the arguments presented there can be adopted literally, on condition that the obvious substitutions are observed, to implement it in the present reduced setting as well.

Proposition 4.2. A restricted $\mathfrak{K}_{0}^{c}$-particle weight $\left(\underline{\pi}, \underline{\mathcal{H}}, \underline{\mathfrak{A}}, \underline{\alpha}, \underline{\mathrm{P}}^{c}, \mathfrak{K}_{0}^{c}\right)$ has the Cluster Property presented in Proposition 3.18, with the reservation that the replacements $\mathfrak{L}_{0} \longmapsto \mathfrak{K}_{0}^{c}$ and $\mathfrak{A} \longmapsto \underline{\mathfrak{A}}^{c}$ have to be carried out. 
Remark. The rather intricate Definition 4.1 will find its justification in the subsequent section, where it turns out, that the characteristics listed above are exactly those which survive in the process of spatial disintegration - at least, it did regrettably not lie within our reach to establish a more complete list of features to be preserved. Nevertheless, it should be noted, that those characteristics motivating the interpretation of particle weights as asymptotic plane waves are perpetuated (cf. the first paragraph of Section 3.3).

Now, it does not come as a surprise that, with respect to the countable and separable notions introduced in Section 4.1, a particle weight of the general type gives rise to a restricted $\overline{\mathfrak{L}_{0}^{c}}$-particle weight.

Theorem 4.3. Let $\left(\pi_{w}, \mathcal{H}_{w}\right)$ be the GNS-representation corresponding to a given particle weight $\langle. \mid$.$\rangle according to Theorem 3.15. Then \left(\pi^{\bullet}, \mathcal{H}^{\bullet}, \mathfrak{A}^{\bullet}, \alpha^{\bullet}, \mathrm{P}^{c}, \overline{\mathfrak{L}_{0}^{c}}\right)$ is a restricted $\overline{\mathfrak{L}_{0}^{c}}$-particle weight, where the individual entries (if not already fixed by Section 4.1) are defined as follows:

- $\mathcal{H}^{\bullet}$ designates the Hilbert subspace of $\mathcal{H}_{w}$, which is the closed $\mathbb{C}$-linear span of the assortment of vectors $\left\{|L\rangle \in \mathcal{H}_{w}: L \in \mathfrak{L}^{c}=\overline{\mathfrak{A}^{c}} \overline{\mathfrak{L}_{0}^{c}}\right\}$ and thus separable;

$-\pi^{\bullet} \doteq \pi_{w}\left\lceil\mathfrak{A}^{\bullet}\right.$ denotes the restriction of the initial representation to the algebra $\mathfrak{A}^{\bullet}$, where the representatives have their limited domain as well as range on $\mathcal{H}^{\bullet}$;

- $\left\{\alpha_{(\Lambda, x)}^{\bullet} \doteq \alpha_{(\Lambda, x)}\left\lceil\mathfrak{A}^{\bullet}:(\Lambda, x) \in \mathrm{P}_{+}^{\uparrow}\right\}\right.$ is the restriction of the initial automorphism group to $\mathfrak{A}^{\bullet}$.

Proof. With the definitions $|.\rangle^{\bullet} \doteq|\rangle \mid. \mathfrak{L}^{c}$ and $U^{\bullet}(x) \doteq U_{w}(x)\left\lceil\mathcal{H}^{\bullet}, x \in \mathbb{R}^{s+1}\right.$, where the latter obviously leaves invariant $\mathcal{H}^{\bullet}$ and is such that the corresponding spectral measure turns out to be $E^{\bullet}(\Delta) \doteq E_{w}(\Delta)\left\lceil\mathcal{H}^{\bullet}\right.$ for any Borel set $\Delta$, all features of the restricted $\overline{\mathfrak{L}_{0}^{c}}$-particle weight are readily checked on the grounds of Theorem 3.15 and Proposition 3.17.

\subsection{Spatial Disintegration of Particle Weights}

We now get to the central result of this chapter: the construction of the spatial disintegration of a particle weight in terms of pure ones, or rather of the corresponding restricted $\overline{\mathfrak{L}_{0}^{c}}$-particle weight into a direct integral of pure representations, which again are associated with restricted $\overline{\mathfrak{L}_{0}^{c}}$-particle weights. In Theorem 4.3 the representation $\left(\pi^{\bullet}, \mathcal{H}^{\bullet}\right)$ of the norm-separable $C^{*}$-algebra $\mathfrak{A}^{\bullet}$ on the separable Hilbert space $\mathcal{H}^{\bullet}$ was derived from the given particle weight $\langle. \mid$.$\rangle . This places the method of spatial disin-$ tegration expounded in the relevant literature at our disposal to apply it to the problem at hand. In order to express $\pi^{\bullet}$ in terms of an integral of irreducible representations, a last preparatory step has to be taken: a maximal abelian von Neumann algebra $\mathfrak{M}$ in the commutant of $\pi^{\bullet}\left(\mathfrak{A}^{\bullet}\right)$ has to be selected in view of [25, Theorem 8.5.2]. The choice of such an algebra is restricted by our further objective to arrive at a disintegration in terms of restricted $\overline{\mathfrak{L}_{0}^{c}}$-particle weights, which means that one has to provide for the possibility to establish the relations (4.9).

The unitary group $\left\{U^{\bullet}(x): x \in \mathbb{R}^{s+1}\right\}$ has generators with joint spectrum in a displaced forward light cone. Through multiplication by suitably chosen exponential 
factors $\exp (i q x)$ with fixed $q \in \bar{V}_{+}$we can pass to another unitary group which likewise implements the space-time translations but has spectrum contained in $\bar{V}_{+}$. This places [9, Theorem IV.5] at our disposal, implying that one can find a strongly continuous unitary group of this kind with elements belonging to $\pi^{\bullet}\left(\mathfrak{A}^{\bullet}\right)^{\prime \prime}$, the weak closure of $\pi^{\bullet}\left(\mathfrak{A}^{\bullet}\right)$ (cf. [11, Corollary 2.4.15]). This result can again be tightened up by use of [10, Theorem 3.3] in the sense that among all the unitary groups complying with the above features there exists exactly one which is characterized by the further requirement that the lower boundary of the joint spectrum of its generators be Lorentz invariant. It is denoted as

$$
\left\{U_{c}^{\bullet}(x) \in \pi^{\bullet}\left(\mathfrak{A}^{\bullet}\right)^{\prime \prime}: x \in \mathbb{R}^{s+1}\right\} .
$$

At this point it turns out to be significant that the $C^{*}$-algebra $\mathfrak{A}^{\bullet}$ has been constructed in Section 4.1 by using local operators so that the reasoning given in [10] applies to the present situation. Another unitary group can be defined through

$$
\left\{V^{\bullet}(x) \doteq U_{c}^{\bullet}(x) U^{\bullet}(x)^{-1}: x \in \mathbb{R}^{s+1}\right\} .
$$

By their very construction, all the operators $V^{\bullet}(x), x \in \mathbb{R}^{s+1}$, are elements of $\pi^{\bullet}\left(\mathfrak{A}^{\bullet}\right)^{\prime}$. The maximal commutative von Neumann algebra $\mathfrak{M}$ that we are going to work with in the sequel is now selected in compliance with the condition

$$
\left\{V^{\bullet}(x): x \in \mathbb{R}^{s+1}\right\}^{\prime \prime} \subseteq \mathfrak{M} \subseteq\left(\pi^{\bullet}\left(\mathfrak{A}^{\bullet}\right) \cup\left\{U^{\bullet}(x): x \in \mathbb{R}^{s+1}\right\}\right)^{\prime} .
$$

The main result to be acquired in the present chapter can then be summarized in the subsequent theorem.

Theorem 4.4. Let $\langle. \mid$.$\rangle be a generic particle weight with representation \left(\pi_{w}, \mathcal{H}_{w}\right)$ inducing, by Theorem 4.3 , the restricted $\overline{\mathfrak{L}_{0}^{c}}$-particle weight $\left(\pi^{\bullet}, \mathcal{H}^{\bullet}, \mathfrak{A}^{\bullet}, \alpha^{\bullet}, \mathrm{P}^{c}, \overline{\mathfrak{L}_{0}^{c}}\right)$. With respect to the representation $\left(\pi^{\bullet}, \mathcal{H}^{\bullet}\right)$ of the separable $C^{*}$-algebra $\mathfrak{A}^{\bullet}$ on the separable Hilbert space $\mathcal{H}^{\bullet}$, we select a maximal abelian von Neumann algebra $\mathfrak{M}$ such that (4.11) is fulfilled. Then there exist a standard Borel space X, a bounded positive measure $\mathrm{v}$ on $\mathrm{X}$, and a field of restricted $\overline{\mathfrak{L}_{0}^{c}}$-particle weights

$$
\mathrm{X} \ni \xi \mapsto\left(\pi_{\xi}, \mathcal{H}_{\xi}, \mathfrak{A}^{\bullet}, \alpha^{\bullet}, \mathrm{P}^{c}, \overline{\mathfrak{L}_{0}^{c}}\right),
$$

such that the following assertions hold true:

(i) The field $\xi \mapsto\left(\pi_{\xi}, \mathcal{H}_{\xi}\right)$, as part of (4.12), is a v-measurable field of irreducible representations of $\mathfrak{A}^{\bullet}$.

(ii) The non-zero representation $\left(\pi^{\bullet}, \mathcal{H}^{\bullet}\right)$ is unitarily equivalent to the direct integral of this field of irreducible representations:

$$
\left(\pi^{\bullet}, \mathcal{H}^{\bullet}\right) \simeq \int_{\mathrm{X}}^{\oplus} d v(\xi)\left(\pi_{\xi}, \mathcal{H}_{\xi}\right)
$$

and, when $W$ denotes the unitary operator connecting both sides of (4.13a), the vectors in both spaces are linked up by the relation

$$
W|L\rangle \cdot=\left\{|L\rangle_{\xi}: \xi \in X\right\} \doteq \int_{X}^{\oplus} d v(\xi)|L\rangle_{\xi}, \quad L \in \mathfrak{L}^{c} \cup \overline{\mathfrak{L}_{0}},
$$

where $|.\rangle_{\xi}$ denotes the linear mapping characteristic for the restricted $\overline{\mathfrak{L}_{0}^{c}}$-particle weight $\left(\pi_{\xi}, \mathcal{H}_{\xi}, \mathfrak{A}^{\bullet}, \alpha^{\bullet}, \mathrm{P}^{c}, \overline{\mathfrak{L}_{0}^{c}}\right)$, according to (4.7a) in Definition 4.1. 
(iii) The von Neumann algebra $\mathfrak{M}$ coincides with the algebra of those operators which are diagonalisable with respect to the above disintegration of $\left(\pi^{\bullet}, \mathcal{H}^{\bullet}\right)$ : any operator $T \in \mathfrak{M}$ corresponds to an essentially bounded measurable complex-valued function $g_{T}$ according to

$$
W T W^{*}=\int_{X}^{\oplus} d v(\xi) g_{T}(\xi) \mathbf{1}_{\xi}
$$

where $\mathbf{1}_{\xi}, \xi \in X$, are the unit operators of the algebras $\mathfrak{B}\left(\mathcal{H}_{\xi}\right)$, respectively.

(iv) Let $\left\{U_{\xi}(x): x \in \mathbb{R}^{s+1}\right\} \subseteq \mathfrak{B}\left(\mathcal{H}_{\xi}\right)$ denote the unitary group, which implements the space-time translations in the restricted $\overline{\mathfrak{L}_{0}^{c}}$-particle weight pertaining to $\xi \in \mathrm{X}$ according to (4.9a), and let $E_{\xi}(\Delta) \in \mathfrak{B}\left(\mathcal{H}_{\xi}\right)$ designate the corresponding spectral measure belonging to the Borel set $\Delta \subseteq \mathbb{R}^{s+1}$. Then the fields of operators

$$
\xi \mapsto U_{\xi}(x) \quad \text { and } \quad \xi \mapsto E_{\xi}(\Delta)
$$

are measurable and satisfy for any $x$ and any Borel set $\Delta$ the following equations:

$$
\begin{aligned}
W U^{\bullet}(x) W^{*} & =\int_{X}^{\oplus} d v(\xi) U_{\xi}(x), \\
W E^{\bullet}(\Delta) W^{*} & =\int_{X}^{\oplus} d v(\xi) E_{\xi}(\Delta) .
\end{aligned}
$$

(v) There exists a canonical choice of a strongly continuous unitary group in each Hilbert space $\mathcal{H}_{\xi}$

$$
\left\{U_{\xi}^{c}(x) \in \pi_{\xi}\left(\mathfrak{A}^{\bullet}\right)^{\prime \prime}=\mathfrak{B}\left(\mathcal{H}_{\xi}\right): x \in \mathbb{R}^{s+1}\right\},
$$

which is measurable with respect to $\xi$, implements the space-time translations in the representation $\left(\pi_{\xi}, \mathcal{H}_{\xi}\right)$ and has generators $P_{\xi}^{c}$ whose joint spectrum lies in the closed forward light cone $\bar{V}_{+}$. It is defined by

$$
U_{\xi}^{c}(x) \doteq \exp \left(i p_{\xi} x\right) U_{\xi}(x), \quad x \in \mathbb{R}^{s+1},
$$

where $p_{\xi}$ is the unequivocal vector in $\mathbb{R}^{s+1}$ that is to be interpreted as the sharp energymomentum corresponding to the respective particle weight.

Remark. The concepts occurring in the theory of direct integrals of Hilbert spaces (standard Borel space, decomposable and diagonalisable operators, and the like) are expounded in [4, Chapter 3], [24, Part II] and likewise [54, Section IV.8 and Appendix].

Proof. The presuppositions of this theorem meet the requirements for an application of [25, Theorem 8.5.2]. This supplies us with

- a standard Borel space $\bar{X}$,

- a bounded positive measure $\bar{v}$ on $\bar{X}$,

- a $\bar{v}$-measurable field $\xi \mapsto\left(\pi_{\xi}, \mathcal{H}_{\xi}\right)$ on $\bar{X}$ consisting of irreducible representations $\pi_{\xi}$ of the $C^{*}$-algebra $\mathfrak{A}^{\bullet}$ on the Hilbert spaces $\mathcal{H}_{\xi}$, 
- and an isomorphism (a linear isometry) $\bar{W}$ from $\mathcal{H}^{\bullet}$ onto the direct integral of these Hilbert spaces, such that

$$
\bar{W}: \mathcal{H}^{\bullet} \rightarrow \int_{\bar{X}}^{\oplus} d \bar{v}(\xi) \mathcal{H}_{\xi},
$$

transforms $\pi^{\bullet}$ into the direct integral of the representations $\pi_{\xi}$ according to

$$
\bar{W} \pi^{\bullet}(A) \bar{W}^{*}=\int_{\overline{\mathrm{X}}}^{\oplus} d \bar{\nu}(\xi) \pi_{\xi}(A), \quad A \in \mathfrak{A}^{\bullet},
$$

and the maximal abelian von Neumann algebra $\mathfrak{M}$ can be identified with the algebra of diagonalisable operators via

$$
\bar{W} T \bar{W}^{*}=\int_{\bar{X}}^{\oplus} d \bar{v}(\xi) g_{T}(\xi) \mathbf{1}_{\xi}, \quad T \in \mathfrak{M},
$$

with an appropriate function $g_{T} \in L^{\infty}(\overline{\mathrm{X}}, d \overline{\mathrm{v}}(\xi))$.

At first sight, the different statements of [25, Theorem 8.5.2] listed above seem to cover almost all of the assertions of the present Theorem 4.4, but one must not forget that the disintegration is to be expressed in terms of a field of restricted $\overline{\mathfrak{L}_{0}^{c}}$-particle weights. So we are left with the task to establish their defining properties in the representations $\left(\pi_{\xi}, \mathcal{H}_{\xi}\right)$ supplied by the standard disintegration theory. In accomplishing this assignment, one has to see to it that simultaneously relation (4.13b) is to be satisfied, which means that one is faced with the following problem: In general the isomorphism $\bar{W}$ connects a given vector $\Psi \in \mathcal{H}^{\bullet}$ not with a unique vector field $\left\{\Psi_{\xi}: \xi \in X\right\}$ but rather with an equivalence class of such fields, characterized by the fact that its elements differ pairwise at most on $\bar{v}$-null sets. In contrast to this, (4.13b) associates the vector field $\left\{|L\rangle_{\xi}: \xi \in X\right\}$ with $|L\rangle^{\bullet}$ for any $L \in \mathfrak{L}^{c} \cup \overline{\mathfrak{L}_{0}}$, leaving no room for any ambiguity. In particular, the algebraic relations prevailing in the set $\mathfrak{L}^{c} \cup \overline{\mathfrak{L}_{0}}$ which carry over to $|$. have to be observed in defining each of the mappings $|.\rangle_{\xi}$ which are characteristic of a restricted $\overline{\mathfrak{L}_{0}^{c}}$-particle weight. The contents of the theorem quoted above, important as they are, can therefore only serve as the starting point for the constructions carried out below, in the course of which again and again $\bar{v}$-null sets have to be removed from $\bar{X}$ to secure definiteness of the remaining components in the disintegration of a given vector. In doing so, one has to be cautious not to apply this procedure uncountably many times; for, otherwise, by accident the standard Borel space $X \subseteq \bar{X}$ arising in the end could happen to be itself a $\bar{v}$-null set. Then, if $v$ denotes the restriction of $\bar{v}$ to this set, one would have $\bar{v}(X)=v(X)=0$, in contradiction to the disintegration (4.13a) of the non-zero representation $\left(\pi^{\bullet}, \mathcal{H}^{\bullet}\right)$.

(i) As indicated above, our first task in view of (4.7a) and (4.7b) of Definition 4.1 will be to establish the existence of $(\mathbb{Q}+i \mathbb{Q})$-linear mappings

$$
|\cdot\rangle_{\xi}: \mathfrak{L}^{c} \rightarrow \mathcal{H}_{\xi}^{c} \quad L \mapsto|L\rangle_{\xi},
$$

from $\mathfrak{L}^{c}$ onto a dense subset $\mathcal{H}_{\xi}^{c}$ of each of the component Hilbert spaces supplied by [25, Theorem 8.5.2] with the property

$$
\pi_{\xi}(A)|L\rangle_{\xi}=|A L\rangle_{\xi}, \quad A \in \overline{\mathfrak{A}^{c}}, \quad L \in \mathfrak{L}^{c} .
$$


Now, by relation (4.14a), there exists to each $L \in \mathfrak{L}^{c}$ an equivalence class of vector fields on $\mathrm{X}$ which corresponds to the element $|L\rangle^{\bullet}$ in $\mathcal{H}^{\bullet}$. The assumed $(\mathbb{Q}+i \mathbb{Q})$ linearity of the mapping $|.\rangle^{\bullet}: \mathfrak{L}^{c} \rightarrow \mathcal{H}^{\bullet}$ carries first of all over to these equivalence classes, but, upon selection of a single representative from each class, it turns out that every algebraic relation in question is fulfilled in all components of the representatives involved, possibly apart from those pertaining to a $\bar{v}$-null set. So, if we pick out one representative of the vector $|L\rangle \bullet$ for every $L$ in the numerable set $\mathfrak{L}^{c}$ and designate it as $\left\{|L\rangle_{\xi}: \xi \in X\right\}$, all of the countably many relations that constitute $(\mathbb{Q}+i \mathbb{Q})$-linearity are satisfied for $\bar{v}$-almost all of the components of these representatives. They can thus be taken to define the linear mappings of the form (4.15a) for all $\xi$ in a Borel subset $\bar{X}_{1}$ of $\bar{X}$, which is left by the procedure of dismissing an appropriate $\bar{v}$-null set for each algebraic relation to be satisfied.

The same reasoning can be applied to the disintegration of vectors of the form $|A L\rangle^{\bullet}=\pi^{\bullet}(A)|L\rangle^{\bullet}$ with $A \in \overline{\mathfrak{A}^{c}}$ and $L \in \mathfrak{L}^{c}$. Again with (4.13b) in mind, the number of relations (4.15b) to be satisfied is countable, so that in view of relation (4.14b) the mere removal of an appropriate $\bar{v}$-null set from $\bar{X}_{1}$ leaves only those indices $\xi$ behind, for which the mappings $|.\rangle_{\xi}$ indeed have the desired property (4.15b).

In this way we have implemented by hand the first defining property of restricted $\overline{\mathfrak{L}_{0}^{c}}$-particle weights in the representations $\left(\pi_{\xi}, \mathcal{H}_{\xi}\right)$ for $\bar{v}$-almost all indices $\xi$. The only thing that remains to be done in this connection is to show that $\left\{|L\rangle_{\xi}: L \in \mathfrak{L}^{c}\right\}$ is a dense subset $\mathcal{H}_{\xi}^{c}$ in $\mathcal{H}_{\xi}$. But, according to [24, Section II.1.6, Proposition 8], the fact that the set $\left\{|L\rangle^{\bullet}: L \in \mathfrak{L}^{c}\right\}$ is total in $\mathcal{H}^{\bullet}$ by assumption implies that the corresponding property holds for $\bar{v}$-almost all $\xi$ in the disintegration. Thus there exists a non-null Borel set $\bar{X}_{2} \subseteq \bar{X}_{1}$, such that the corresponding mappings $|.\rangle_{\xi}, \xi \in \bar{X}_{2}$, have this property, too. In this way all of the characteristics presented in the first item of Definition 4.1 are fulfilled for $\xi \in \bar{X}_{2}$ by the mappings (4.15a) constructed above, and additionally we have

$$
\bar{W}|L\rangle \cdot \int_{\bar{X}_{2}}^{\oplus} d \bar{v}(\xi)|L\rangle_{\xi}, \quad L \in \mathfrak{L}^{c} .
$$

(ii) In the next step, the mappings $|.\rangle_{\xi}$ have to be extended to the set $\overline{\mathfrak{L}_{0}}$ of all Poincaré transforms of operators from $\overline{\mathfrak{L}_{0}^{c}}$ in such a way that the counterpart of (4.8) in Definition 4.1 is continuous. In the present notation this is the mapping

$$
\mathrm{P}_{+}^{\uparrow} \ni(\Lambda, x) \mapsto\left|\alpha_{(\Lambda, x)}^{\bullet}\left(L^{\prime}\right)\right\rangle_{\xi} \in \mathcal{H}_{\xi}, \quad L^{\prime} \in \overline{\mathfrak{L}_{0}}
$$

At this point the special selection of $\overline{\mathfrak{L}_{0}^{c}}$ as consisting of compactly regularized vacuum annihilation operators comes into play, and also the invariance of this set under transformations $(\Lambda, x) \in \overline{\mathrm{P}}^{c}$ will be of importance. Great care has to be taken in these investigations based on the differentiability properties of the operators in question, that not uncountably many conditions are imposed on the mappings $|.\rangle_{\xi}$, since anew not all of them will share the claimed extension property, but only a $\bar{v}$-null subset of $\bar{X}_{2}$ shall get lost on the way.

To start with, note that the Poincare group $\mathrm{P}_{+}^{\uparrow}$ can be covered by a sequence of open sets $\mathrm{V}_{i}$ with compact closures $\mathrm{C}_{i}, i \in \mathbb{N}$, contained in corresponding open charts $\left(\mathrm{U}_{i}, \phi_{i}\right)$ with the additional property that the sets $\phi_{i}\left(C_{i}\right) \subseteq \mathbb{R}^{d_{\mathrm{P}}}$ are convex (e.g. consider the 
translates of the canonical coordinates $\left(\mathrm{U}_{0}, \phi_{0}\right)$ around $(\mathbf{1}, 0)$ to all elements of $\mathrm{P}^{c}$ and take suitable open subsets thereof). Select one of these compacta, say $\mathrm{C}_{k}$, and fix an element $\hat{L}_{0} \in \overline{\mathfrak{L}_{0}^{c}}$, which by assumption is given as a compactly supported regularization of an element $L_{0} \in \mathfrak{L}_{0}^{c}$ :

$$
\hat{L}_{0}=\alpha_{F}\left(L_{0}\right) \doteq \int_{\mathrm{S}_{F}} d \mu(\Lambda, x) F(\Lambda, x) \alpha_{(\Lambda, x)}\left(L_{0}\right)
$$

where $F$ is an infinitely often differentiable function on $\mathrm{P}_{+}^{\uparrow}$ with compact support $\mathrm{S}_{F}$ in the Poincaré group $\mathrm{P}_{+}^{\uparrow}$. According to Lemma 3.16 the mapping $\mid$. $\rangle$ commutes with this integral so that the vector $\left|\hat{L}_{0}\right\rangle$ in $\mathcal{H}_{w}$ takes on the shape

$$
\left|\hat{L}_{0}\right\rangle=\int_{\mathrm{S}_{F}} d \mu(\Lambda, x) F(\Lambda, x)\left|\alpha_{(\Lambda, x)}\left(L_{0}\right)\right\rangle .
$$

The same equation holds for the Poincare transforms of the operator $\hat{L}_{0}$ as well, so that invariance of the Haar measure on $\mathrm{P}_{+}^{\uparrow}$ implies for any $\left(\Lambda_{0}, x_{0}\right) \in \mathrm{C}_{k}$ the equations

$$
\begin{aligned}
&\left|\alpha_{\left(\Lambda_{0}, x_{0}\right)}\left(\hat{L}_{0}\right)\right\rangle=\int_{\mathrm{S}_{F}} d \mu(\Lambda, x) F(\Lambda, x)\left|\alpha_{\left(\Lambda_{0}, x_{0}\right)(\Lambda, x)}\left(L_{0}\right)\right\rangle \\
&=\int_{\left(\Lambda_{0}, x_{0}\right) \cdot \mathrm{S}_{F}} d \mu(\Lambda, x) F\left(\left(\Lambda_{0}, x_{0}\right)^{-1}(\Lambda, x)\right)\left|\alpha_{(\Lambda, x)}\left(L_{0}\right)\right\rangle \\
& \quad=\int_{\mathrm{C}_{k} \cdot \mathrm{S}_{F}} d \mu(\Lambda, x) F\left(\left(\Lambda_{0}, x_{0}\right)^{-1}(\Lambda, x)\right)\left|\alpha_{(\Lambda, x)}\left(L_{0}\right)\right\rangle .
\end{aligned}
$$

The derivatives of the mapping $\left(\Lambda_{0}, x_{0}\right) \mapsto\left|\alpha_{\left(\Lambda_{0}, x_{0}\right)}\left(\hat{L}_{0}\right)\right\rangle$, the domain of $\left(\Lambda_{0}, x_{0}\right)$ restricted to the neighbourhood $\mathrm{V}_{k}$ in $\mathrm{C}_{k}$, are thus explicitly seen to be expressible in terms of derivatives of the functions

$$
F^{(\Lambda, x)}: \bigvee_{k} \rightarrow \mathbb{C} \quad\left(\Lambda_{0}, x_{0}\right) \mapsto F^{(\Lambda, x)}\left(\Lambda_{0}, x_{0}\right) \doteq F\left(\left(\Lambda_{0}, x_{0}\right)^{-1}(\Lambda, x)\right)
$$

So, let $\left(\Lambda_{1}, x_{1}\right)$ and $\left(\Lambda_{2}, x_{2}\right)$ be a pair of Poincaré transformations lying in the common neighbourhood $\mathrm{V}_{k}$; then the following equation results from an application of the Mean Value Theorem A.7 to the $X_{\mathfrak{L}_{0}}$-differentiable mapping $\mid$. $\rangle$ (cf. Theorem 3.15):

$$
\begin{aligned}
& \left|\alpha_{\left(\Lambda_{1}, x_{1}\right)}\left(\hat{L}_{0}\right)-\alpha_{\left(\Lambda_{2}, x_{2}\right)}\left(\hat{L}_{0}\right)\right\rangle \\
& =\left|\alpha_{\phi_{k}^{-1}(s)}\left(\hat{L}_{0}\right)-\alpha_{\phi_{k}^{-1}(t)}\left(\hat{L}_{0}\right)\right\rangle=\int_{0}^{1} d \vartheta\left|\mathfrak{D}\left(\Xi_{\hat{L}_{0}} \circ \phi_{k}^{-1}\right)(\boldsymbol{t}+\vartheta(\boldsymbol{s}-\boldsymbol{t}))(\boldsymbol{s}-\boldsymbol{t})\right\rangle \\
& \quad=\int_{0}^{1} d \vartheta \int_{C_{k} \cdot S_{F}} d \mu(\Lambda, x) \mathfrak{D}\left(F^{(\Lambda, x)} \circ \phi_{k}^{-1}\right)(\boldsymbol{t}+\vartheta(\boldsymbol{s}-\boldsymbol{t}))(\boldsymbol{s}-\boldsymbol{t})\left|\alpha_{(\Lambda, x)}\left(L_{0}\right)\right\rangle,
\end{aligned}
$$

where $\boldsymbol{s} \doteq \phi_{k}\left(\Lambda_{1}, x_{1}\right)$ and $\boldsymbol{t} \doteq \phi_{k}\left(\Lambda_{2}, x_{2}\right)$ belong to the compact and convex set $\phi_{k}\left(C_{k}\right)$.

Now, the vector $\left|\alpha_{\left(\Lambda_{1}, x_{1}\right)}\left(\hat{L}_{0}\right)-\alpha_{\left(\Lambda_{2}, x_{2}\right)}\left(\hat{L}_{0}\right)\right\rangle$ defines a positive functional on the algebra $\mathfrak{B}\left(\mathcal{H}_{w}\right)$, and we want to show that this vector functional can be majorized by a positive normal functional in $\mathfrak{B}(\mathcal{H})_{*}$. To establish this fact, note, that the integrals in (4.18d) exist in the uniform topology of $\mathcal{H}_{w}$, so that they commute with every bounded 
linear operator $B \in \mathfrak{B}\left(\mathcal{H}_{w}\right)$. Hence

$$
\begin{aligned}
& \left\langle\alpha_{\left(\Lambda_{1}, x_{1}\right)}\left(\hat{L}_{0}\right)-\alpha_{\left(\Lambda_{2}, x_{2}\right)}\left(\hat{L}_{0}\right)|B| \alpha_{\left(\Lambda_{1}, x_{1}\right)}\left(\hat{L}_{0}\right)-\alpha_{\left(\Lambda_{2}, x_{2}\right)}\left(\hat{L}_{0}\right)\right\rangle \\
& =\iint_{[0,1]^{2}} d \vartheta d \vartheta^{\prime}\left\langle\mathfrak{D}\left(\Xi_{\hat{L}_{0}} \circ \phi_{k}^{-1}\right)\left(\boldsymbol{t}+\vartheta^{\prime}(\boldsymbol{s}-\boldsymbol{t})\right)(\boldsymbol{s}-\boldsymbol{t})|B| \mathfrak{D}\left(\Xi_{\hat{L}_{0}} \circ \phi_{k}^{-1}\right)(\boldsymbol{t}+\vartheta(\boldsymbol{s}-\boldsymbol{t}))(\boldsymbol{s}-\boldsymbol{t})\right\rangle .
\end{aligned}
$$

This equation is invariant with respect to an exchange of $\vartheta$ and $\vartheta^{\prime}$. In the case of a positive operator $B$ the following relation holds for arbitrary vectors $\Psi$ and $\Phi$ in $\mathcal{H}_{w}$ :

$$
\langle\Psi|B| \Phi\rangle+\langle\Phi|B| \Psi\rangle \leqslant\langle\Psi|B| \Psi\rangle+\langle\Phi|B| \Phi\rangle,
$$

which, applied to the integrand of (4.19a) and to that resulting from an interchange of $\vartheta$ and $\vartheta^{\prime}$, yields

$$
\begin{aligned}
& \left\langle\alpha_{\left(\Lambda_{1}, x_{1}\right)}\left(\hat{L}_{0}\right)-\alpha_{\left(\Lambda_{2}, x_{2}\right)}\left(\hat{L}_{0}\right)|B| \alpha_{\left(\Lambda_{1}, x_{1}\right)}\left(\hat{L}_{0}\right)-\alpha_{\left(\Lambda_{2}, x_{2}\right)}\left(\hat{L}_{0}\right)\right\rangle \\
& \quad \leqslant \int_{0}^{1} d \vartheta\left\langle\mathfrak{D}\left(\Xi_{\hat{L}_{0}} \circ \phi_{k}^{-1}\right)(\boldsymbol{t}+\vartheta(\boldsymbol{s}-\boldsymbol{t}))(\boldsymbol{s}-\boldsymbol{t})|B| \mathfrak{D}\left(\Xi_{\hat{L}_{0}} \circ \phi_{k}^{-1}\right)(\boldsymbol{t}+\vartheta(\boldsymbol{s}-\boldsymbol{t}))(\boldsymbol{s}-\boldsymbol{t})\right\rangle
\end{aligned}
$$

upon execution of a trivial integration over $\vartheta$ and $\vartheta^{\prime}$, respectively. As in (4.18d) we can pass to the following representation for the integrand on the right-hand side of (4.19b):

$$
\begin{aligned}
\left\langle\mathfrak{D}\left(\Xi_{\hat{L}_{0}} \circ \phi_{k}^{-1}\right)(\boldsymbol{t}+\vartheta(\boldsymbol{s}-\boldsymbol{t}))(\boldsymbol{s}-\boldsymbol{t})|B| \mathfrak{D}\left(\Xi_{\hat{L}_{0}} \circ \phi_{k}^{-1}\right)(\boldsymbol{t}+\vartheta(\boldsymbol{s}-\boldsymbol{t}))(\boldsymbol{s}-\boldsymbol{t})\right\rangle \\
=\int_{\mathrm{C}_{k} \cdot \mathrm{S}_{F}} d \mu(\Lambda, x) \int_{\mathrm{C}_{k} \cdot \mathrm{S}_{F}} d \mu\left(\Lambda^{\prime}, x^{\prime}\right) \overline{\mathfrak{D}\left(F^{\left(\Lambda^{\prime}, x^{\prime}\right)} \circ \phi_{k}^{-1}\right)(\boldsymbol{t}+\vartheta(\boldsymbol{s}-\boldsymbol{t}))(\boldsymbol{s}-\boldsymbol{t})} . \\
\cdot \mathfrak{D}\left(F^{(\Lambda, x)} \circ \phi_{k}^{-1}\right)(\boldsymbol{t}+\vartheta(\boldsymbol{s}-\boldsymbol{t}))(\boldsymbol{s}-\boldsymbol{t})\left\langle\alpha_{\left(\Lambda^{\prime}, x^{\prime}\right)}\left(L_{0}\right)|B| \alpha_{(\Lambda, x)}\left(L_{0}\right)\right\rangle .
\end{aligned}
$$

The derivatives which show up in (4.19c) depend by construction continuously on the parameters $\boldsymbol{s}$ and $\boldsymbol{t}, \vartheta$ and $\vartheta^{\prime}$ as well as $(\Lambda, x)$ and $\left(\Lambda^{\prime}, x^{\prime}\right)$, so that their absolute values, taken on the compact domains $\phi_{k}\left(C_{k}\right),[0,1]$ and $C_{k} \cdot S_{F}$, respectively, are bounded by

$$
\left|\mathfrak{D}\left(F^{(\Lambda, x)} \circ \phi_{k}^{-1}\right)(\boldsymbol{t}+\vartheta(\boldsymbol{s}-\boldsymbol{t}))(\boldsymbol{s}-\boldsymbol{t})\right| \leqslant D\left(F ; C_{k}\right)|\boldsymbol{s}-\boldsymbol{t}|<\infty
$$

for all $(\Lambda, x) \in \mathrm{C}_{k} \cdot \mathrm{S}_{F}$ with a suitable non-negative constant $D\left(F ; \mathrm{C}_{k}\right)$. Hence the nonnegative matrix element in (4.19c) can be estimated by

$$
\begin{aligned}
& \left\langle\mathfrak{D}\left(\Xi_{\hat{L}_{0}} \circ \phi_{k}^{-1}\right)(\boldsymbol{t}+\vartheta(\boldsymbol{s}-\boldsymbol{t}))(\boldsymbol{s}-\boldsymbol{t})|B| \mathfrak{D}\left(\Xi_{\hat{L}_{0}} \circ \phi_{k}^{-1}\right)(\boldsymbol{t}+\vartheta(\boldsymbol{s}-\boldsymbol{t}))(\boldsymbol{s}-\boldsymbol{t})\right\rangle \\
& \quad \leqslant D\left(F ; C_{k}\right)^{2}|\boldsymbol{s}-\boldsymbol{t}|^{2} \int_{\mathrm{C}_{k} \cdot S_{F}} d \mu(\Lambda, x) \int_{\mathrm{C}_{k} \cdot S_{F}} d \mu\left(\Lambda^{\prime}, x^{\prime}\right)\left|\left\langle\alpha_{\left(\Lambda^{\prime}, x^{\prime}\right)}\left(L_{0}\right)|B| \alpha_{(\Lambda, x)}\left(L_{0}\right)\right\rangle\right|,
\end{aligned}
$$

which is independent of $\vartheta$, so that insertion into (4.19b) yields

$$
\begin{aligned}
& \left\langle\alpha_{\left(\Lambda_{1}, x_{1}\right)}\left(\hat{L}_{0}\right)-\alpha_{\left(\Lambda_{2}, x_{2}\right)}\left(\hat{L}_{0}\right)|B| \alpha_{\left(\Lambda_{1}, x_{1}\right)}\left(\hat{L}_{0}\right)-\alpha_{\left(\Lambda_{2}, x_{2}\right)}\left(\hat{L}_{0}\right)\right\rangle \\
& \quad \leqslant D\left(F ; C_{k}\right)^{2}|\boldsymbol{s}-\boldsymbol{t}|^{2} \int_{\mathrm{C}_{k} \cdot \mathrm{S}_{F}} d \mu(\Lambda, x) \int_{\mathrm{C}_{k} \cdot \mathrm{S}_{F}} d \mu\left(\Lambda^{\prime}, x^{\prime}\right)\left|\left\langle\alpha_{\left(\Lambda^{\prime}, x^{\prime}\right)}\left(L_{0}\right)|B| \alpha_{(\Lambda, x)}\left(L_{0}\right)\right\rangle\right| .
\end{aligned}
$$


Since the positive operator $B$ can be written as $C^{*} C$ for suitable $C \in \mathfrak{B}\left(\mathcal{H}_{w}\right)$, the integrand on the right-hand side allows for the following estimate, making use of the relation between the geometric and the arithmetic mean of two non-negative numbers:

$$
\begin{aligned}
& \left|\left\langle\alpha_{\left(\Lambda^{\prime}, x^{\prime}\right)}\left(L_{0}\right)|B| \alpha_{(\Lambda, x)}\left(L_{0}\right)\right\rangle\right| \leqslant \| C\left|\alpha_{\left(\Lambda^{\prime}, x^{\prime}\right)}\left(L_{0}\right)\right\rangle\|\||| C\left|\alpha_{(\Lambda, x)}\left(L_{0}\right)\right\rangle \| \\
& \leqslant 2^{-1}\left(\left\langle\alpha_{\left(\Lambda^{\prime}, x^{\prime}\right)}\left(L_{0}\right)|B| \alpha_{\left(\Lambda^{\prime}, x^{\prime}\right)}\left(L_{0}\right)\right\rangle+\left\langle\alpha_{(\Lambda, x)}\left(L_{0}\right)|B| \alpha_{(\Lambda, x)}\left(L_{0}\right)\right\rangle\right) .
\end{aligned}
$$

As a consequence of this inequality entered into (4.19e), one integration over $C_{k} \cdot S_{F}$ can be carried out on its right-hand side for each resulting term of the sum, so that finally

$$
\begin{aligned}
& \left\langle\alpha_{\left(\Lambda_{1}, x_{1}\right)}\left(\hat{L}_{0}\right)-\alpha_{\left(\Lambda_{2}, x_{2}\right)}\left(\hat{L}_{0}\right)|B| \alpha_{\left(\Lambda_{1}, x_{1}\right)}\left(\hat{L}_{0}\right)-\alpha_{\left(\Lambda_{2}, x_{2}\right)}\left(\hat{L}_{0}\right)\right\rangle \\
& \quad \leqslant D\left(F ; C_{k}\right)^{2}|s-t|^{2} \mu\left(C_{k} \cdot S_{F}\right) \int_{C_{k} \cdot S_{F}} d \mu(\Lambda, x)\left\langle\alpha_{(\Lambda, x)}\left(L_{0}\right)|B| \alpha_{(\Lambda, x)}\left(L_{0}\right)\right\rangle,
\end{aligned}
$$

where the last integral can be viewed as a positive normal functional on $\mathfrak{B}\left(\mathcal{H}_{w}\right)$ in the variable $B$, as announced at the beginning of this paragraph.

Now, let $M$ be a measurable subset of $\bar{X}$ then, according to (4.14c), it corresponds via the associated characteristic function $\chi_{M}$ to a projection $P_{M}$ in the selected maximal abelian von Neumann algebra $\mathfrak{M}$. If $P^{\bullet}$ in turn denotes the orthogonal projection from $\mathcal{H}_{w}$ onto the Hilbert space $\mathcal{H}^{\bullet}$, we can define $B_{M} \doteq P^{\bullet} P_{M} P^{\bullet}$ as a positive operator in $\mathfrak{B}\left(\mathcal{H}_{w}\right)$, which is therefore subject to (4.19f). This relation can then be re-written for $B=B_{M}$ in terms of the restricted $\overline{\mathfrak{L}_{0}^{c}}$-particle weight $\left(\pi^{\bullet}, \mathcal{H}^{\bullet}, \mathfrak{A}^{\bullet}, \alpha^{\bullet}, \mathrm{P}^{c}, \overline{\mathfrak{L}_{0}^{c}}\right)$ :

$$
\begin{aligned}
\| P_{M}\left|\alpha_{\left(\Lambda_{1}, x_{1}\right)}^{\bullet}\left(\hat{L}_{0}\right)-\alpha_{\left(\Lambda_{2}, x_{2}\right)}^{\bullet}\left(\hat{L}_{0}\right)\right\rangle & \bullet\left\|^{2}=\right\| P_{M} P^{\bullet}\left|\alpha_{\left(\Lambda_{1}, x_{1}\right)}\left(\hat{L}_{0}\right)-\alpha_{\left(\Lambda_{2}, x_{2}\right)}\left(\hat{L}_{0}\right)\right\rangle \|^{2} \\
& \leqslant D\left(F ; C_{k}\right)^{2}|s-\boldsymbol{t}|^{2} \mu\left(\mathrm{C}_{k} \cdot \mathrm{S}_{F}\right) \int_{\mathrm{C}_{k} \cdot \mathrm{S}_{F}} d \mu(\Lambda, x)\left\langle\alpha_{(\Lambda, x)}\left(L_{0}\right)\left|P^{\bullet} P_{M} P^{\bullet}\right| \alpha_{(\Lambda, x)}\left(L_{0}\right)\right\rangle,
\end{aligned}
$$

where now the integral on the right-hand side defines a positive normal functional on the von Neumann algebra $\mathfrak{M}$ through

$$
\varphi\left[\hat{L}_{0} ; \mathrm{C}_{k}\right](T) \doteq \int_{\mathrm{C}_{k} \cdot \mathrm{S}_{F}} d \mu(\Lambda, x)\left\langle\alpha_{(\Lambda, x)}\left(L_{0}\right)\left|P^{\bullet} T P^{\bullet}\right| \alpha_{(\Lambda, x)}\left(L_{0}\right)\right\rangle, \quad T \in \mathfrak{M} .
$$

Specializing to Poincare transformations $\left(\Lambda_{1}, x_{1}\right)$ and $\left(\Lambda_{2}, x_{2}\right)$ from the countable subgroup $\overline{\mathrm{P}}^{c}$, the unique disintegration of the vector $\left|\alpha_{\left(\Lambda_{1}, x_{1}\right)}^{\bullet}\left(\hat{L}_{0}\right)-\alpha_{\left(\Lambda_{2}, x_{2}\right)}^{\bullet}\left(\hat{L}_{0}\right)\right\rangle^{\bullet}$ occurring on the left-hand side of (4.20a) is already explicitly given by (4.15a) for all $\xi \in \overline{\mathrm{X}}_{2}$ so that

$$
\bar{W}\left|\alpha_{\left(\Lambda_{1}, x_{1}\right)}^{\bullet}\left(\hat{L}_{0}\right)-\alpha_{\left(\Lambda_{2}, x_{2}\right)}^{\bullet}\left(\hat{L}_{0}\right)\right\rangle=\int_{\bar{x}_{2}}^{\oplus} d v(\xi)\left|\alpha_{\left(\Lambda_{1}, x_{1}\right)}^{\bullet}\left(\hat{L}_{0}\right)-\alpha_{\left(\Lambda_{2}, x_{2}\right)}^{\bullet}\left(\hat{L}_{0}\right)\right\rangle_{\xi} .
$$

On the other hand, the positive normal functional $\varphi\left[\hat{L}_{0}, C_{k}\right] \in \mathfrak{M}_{*}$ of (4.20b) is easily seen by [54, Proposition IV.8.34] in connection with (4.14c) to correspond to a unique integrable field $\left\{\varphi\left[\hat{L}_{0}, C_{k}\right]_{\xi}: \xi \in \bar{X}\right\}$ of positive normal functionals on the von Neumann algebras $\mathbb{C} \cdot \mathbf{1}_{\xi}$ in the direct integral decomposition of $\mathfrak{M}$. Explicitly,

$$
\varphi\left[\hat{L}_{0} ; C_{k}\right](T)=\int_{\bar{X}} d \bar{v}(\xi) g_{T}(\xi) \varphi\left[\hat{L}_{0}, C_{k}\right]_{\xi}\left(\mathbf{1}_{\xi}\right)
$$


for any $T \in \mathfrak{M}$ with an appropriate function $g_{T} \in L^{\infty}(\overline{\mathrm{X}}, d \overline{\mathrm{v}}(\xi))$. The above relation stays true, if we replace $\bar{X}$ by $\bar{X}_{2}$, since both differ at most by a $\bar{v}$-null set. So, in view of relations (4.20b) through (4.20d), (4.20a) can for any measurable subset $M$ of $\bar{X}_{2}$ corresponding to the orthogonal projection $P_{M} \in \mathfrak{M}$ be expressed in terms of integrals according to

$$
\begin{aligned}
\int_{M} d \overline{\boldsymbol{v}}(\xi) \|\left|\alpha_{\left(\Lambda_{1}, x_{1}\right)}^{\bullet}\left(\hat{L}_{0}\right)-\alpha_{\left(\Lambda_{2}, x_{2}\right)}^{\bullet}\left(\hat{L}_{0}\right)\right\rangle_{\xi} \|^{2} & \\
& \leqslant D\left(F ; C_{k}\right)^{2}|\boldsymbol{s}-\boldsymbol{t}|^{2} \mu\left(\mathrm{C}_{k} \cdot \mathrm{S}_{F}\right) \int_{M} d \bar{v}(\xi) \varphi\left[\hat{L}_{0}, C_{k}\right]_{\xi}\left(\mathbf{1}_{\xi}\right) .
\end{aligned}
$$

Due to arbitrariness of $M \subseteq \bar{X}_{2}$, we then infer, making use of elementary results of integration theory [35, Chapter V, viz. $\S 25$, Theorem D], that for $\bar{v}$-almost all $\xi \in \bar{X}_{2}$ there holds the estimate

$$
\begin{aligned}
& \|\left|\alpha_{\left(\Lambda_{1}, x_{1}\right)}^{\bullet}\left(\hat{L}_{0}\right)-\alpha_{\left(\Lambda_{2}, x_{2}\right)}^{\bullet}\left(\hat{L}_{0}\right)\right\rangle_{\xi} \| \\
& \quad \leqslant\left|\phi_{k}\left(\Lambda_{1}, x_{1}\right)-\phi_{k}\left(\Lambda_{2}, x_{2}\right)\right| \cdot D\left(F ; C_{k}\right) \cdot \mu\left(C_{k} \cdot S_{F}\right) \cdot \varphi\left[\hat{L}_{0}, C_{k}\right]_{\xi}\left(\mathbf{1}_{\xi}\right)
\end{aligned}
$$

where we replaced the points $\boldsymbol{s}$ and $\boldsymbol{t}$ from the space $\mathbb{R}^{d_{\mathrm{P}}}$ of coordinates for $\mathrm{P}_{+}^{\uparrow}$ by their pre-images $\left(\Lambda_{1}, x_{1}\right)$ and $\left(\Lambda_{2}, x_{2}\right)$ from $\bigvee_{k} \cap \overline{\mathrm{P}}^{c}$. The important thing to notice at this point is that, apart from the factor $\left|\phi_{k}\left(\Lambda_{1}, x_{1}\right)-\phi_{k}\left(\Lambda_{2}, x_{2}\right)\right|$, the terms on the righthand side of (4.20f) hinge upon the operator $\hat{L}_{0}$ (determining the function $F$ as well as its support $S_{F}$ ) and on the neighbourhood $V_{k}$ with compact closure $C_{k}$ containing $\left(\Lambda_{1}, x_{1}\right),\left(\Lambda_{2}, x_{2}\right) \in \overline{\mathrm{P}}^{c}$. Therefore this estimate also holds for any other pair of Lorentz transformations in $\mathrm{V}_{k} \cap \overline{\mathrm{P}}^{c}$ with the same $\left(\hat{L}_{0}, \mathrm{~V}_{k}\right)$-dependent factor; of course, in each of the resulting countably many relations one possibly loses a further $\bar{v}$-null subset of $\bar{X}_{2}$. The reasoning leading up to this point can then be applied to any combination of an operator in the numerable selection $\overline{\mathfrak{L}_{0}^{c}}$ with an open set from the countable cover of $\mathrm{P}_{+}^{\uparrow}$ to produce in each case a relation of the form of (4.20f) for the respective Poincare transformations in $\overline{\mathrm{P}}^{c}$. Simultaneously, the domain of indices $\xi$, for which all of these inequalities are valid, shrinks to an appropriate $\bar{v}$-measurable non-null subset $\bar{X}_{3}$ of $\bar{X}_{2}$.

Consider now an arbitrary element $\left(\Lambda_{0}, x_{0}\right) \in \mathrm{P}_{+}^{\uparrow}$, which belongs to at least one of the open sets $V_{j}$ from the covering of the Poincare group already used above. By density of $\overline{\mathrm{P}}^{c}$ in $\mathrm{P}_{+}^{\uparrow}$, the transformation $\left(\Lambda_{0}, x_{0}\right)$ can be approximated by a sequence $\left\{\left(\Lambda_{n}, x_{n}\right)\right\}_{n \in \mathbb{N}} \subseteq \overline{\mathrm{P}}^{c} \cap \mathrm{V}_{j}$. This is a Cauchy sequence in the initial topology of $\mathrm{P}_{+}^{\uparrow}$, so that relation (4.20f) implies that for each $\xi \in \bar{X}_{3}$ the corresponding sequences

$$
\left\{\left|\alpha_{\left(\Lambda_{n}, x_{n}\right)}^{\bullet}\left(\hat{L}_{0}\right)\right\rangle_{\xi}\right\}_{n \in \mathbb{N}} \subseteq \mathcal{H}_{\xi}, \quad \hat{L}_{0} \in \overline{\mathfrak{L}_{0}^{c}},
$$

likewise have the Cauchy property with respect to the Hilbert space norms. Their limits in each of the spaces $\mathcal{H}_{\xi}, \xi \in \bar{X}_{3}$, thus exist and are obviously independent of the approximating sequence of Lorentz transformations from $\overline{\mathrm{P}}^{c}$. Therefore, we can write

$$
\left|\hat{L}_{0} ;\left(\Lambda_{0}, x_{0}\right)\right\rangle_{\xi} \doteq \lim _{n \rightarrow \infty}\left|\alpha_{\left(\Lambda_{n}, x_{n}\right)}^{\bullet}\left(\hat{L}_{0}\right)\right\rangle_{\xi}
$$

a result that holds for arbitrary $\hat{L}_{0} \in \overline{\mathfrak{L}_{0}^{c}}$ as long as $\xi$ is taken from the non-null set $\bar{X}_{3}$. According to [26, Definition II.4.1], which lays down the notion of measurability for 
vector fields, the mapping

$$
\bar{X}_{3} \ni \xi \mapsto\left|\hat{L}_{0} ;\left(\Lambda_{0}, x_{0}\right)\right\rangle_{\xi} \in \mathcal{H}_{\xi},
$$

that arises as the pointwise limit of measurable vector fields on $\bar{X}_{3}$, is itself measurable with respect to the restriction of $\bar{v}$ to this subset of $\bar{X}$ and turns out to be a representative of the vector $\left|\alpha_{\left(\Lambda_{0}, x_{0}\right)}^{\bullet}\left(\hat{L}_{0}\right)\right\rangle^{\bullet} \in \mathcal{H}^{\bullet}$ (cf. [24, Section II.1.5, Proof of Proposition 5(ii)], and note that we can neglect the null set missing in $\bar{X}_{3}$ compared to $\left.\bar{X}\right)$.

The question now is, if the limits $\left|\hat{L}_{0} ;\left(\Lambda_{0}, x_{0}\right)\right\rangle_{\xi}$, constructed by the above method for arbitrary operators $\hat{L}_{0} \in \overline{\mathfrak{L}_{0}^{c}}$ and any transformation $\left(\Lambda_{0}, x_{0}\right) \in \mathrm{P}_{+}^{\uparrow}$, can unambiguously be identified for all $\xi$ in $\bar{X}_{3}$ with vectors $\left|\alpha_{\left(\Lambda_{0}, x_{0}\right)}\left(\hat{L}_{0}\right)\right\rangle_{\xi} \in \mathcal{H}_{\xi}$, which satisfy a relation of the form (4.13b). One of the situations, in which an inconsistency possibly arises, is the appearance of two different representations for a single element $L^{\prime} \in \overline{\mathfrak{L}_{0}}$ :

$$
L^{\prime}=\alpha_{\left(\Lambda_{1}, x_{1}\right)}^{\bullet}\left(\hat{L}_{1}\right)=\alpha_{\left(\Lambda_{2}, x_{2}\right)}^{\bullet}\left(\hat{L}_{2}\right),
$$

where $\hat{L}_{1}, \hat{L}_{2} \in \overline{\mathfrak{L}_{0}^{c}}$, and $\left(\Lambda_{1}, x_{1}\right),\left(\Lambda_{2}, x_{2}\right) \in \mathrm{P}_{+}^{\uparrow}$. In this case the pair of operators is connected by the Poincaré transformation $\left(\Lambda_{1}, x_{1}\right)^{-1}\left(\Lambda_{2}, x_{2}\right)$, which belongs to the subgroup $\overline{\mathrm{P}}^{c}$ of $\mathrm{P}_{+}^{\uparrow}$ according to the constructions of Section 4.1. Therefore

$$
\hat{L}_{1}=\alpha_{\left(\Lambda_{1}, x_{1}\right)^{-1}\left(\Lambda_{2}, x_{2}\right)}^{\bullet}\left(\hat{L}_{2}\right),
$$

which implies that

$$
\alpha_{\left(\Lambda_{1, n}, x_{1, n}\right)}^{\bullet}\left(\hat{L}_{1}\right)=\alpha_{\left(\Lambda_{1, n}, x_{1, n}\right)\left(\Lambda_{1}, x_{1}\right)^{-1}\left(\Lambda_{2}, x_{2}\right)}^{\bullet}\left(\hat{L}_{2}\right)
$$

for any sequence $\left\{\left(\Lambda_{1, n}, x_{1, n}\right)\right\}_{n \in \mathbb{N}} \subseteq \overline{\mathrm{P}}^{c}$ approximating $\left(\Lambda_{1}, x_{1}\right)$. But then the transformations on the right-hand side of the last equation constitute another sequence in $\overline{\mathrm{P}}^{c}$, which in this case tends to $\left(\Lambda_{2}, x_{2}\right)$ in the limit $n \rightarrow \infty$. As a consequence of the independence of the limits (4.21b) from the selected sequence in $\overline{\mathrm{P}}^{c}$, we could define

$$
\left|L^{\prime}\right\rangle_{\xi} \doteq\left|\hat{L}_{1} ;\left(\Lambda_{1}, x_{1}\right)\right\rangle_{\xi}=\left|\hat{L}_{2} ;\left(\Lambda_{2}, x_{2}\right)\right\rangle_{\xi}
$$

The only problem that is still left open with respect to an unequivocal definition of vectors of the form $\left|L^{\prime}\right\rangle_{\xi}, L^{\prime} \in \overline{\mathfrak{L}_{0}}$, occurs when the vacuum annihilation operator $L^{\prime}$ happens to be an element of $\mathfrak{L}^{c}$, so that its components in the Hilbert spaces $\mathcal{H}_{\xi}$ have already been fixed in the initial step. But, as $\mathfrak{L}^{c}$ is a numerable set, such a coincidence will be encountered at most countably often, so that relation (4.21e) indeed turns out to be the unique definition of $\left|L^{\prime}\right\rangle_{\xi}$ for all $\xi \in \bar{X}_{4}$, such that the relation

$$
\bar{W}\left|L^{\prime}\right\rangle^{\bullet}=\int_{\overline{\mathrm{X}}_{4}}^{\oplus} d \bar{v}(\xi)\left|L^{\prime}\right\rangle_{\xi}, \quad L^{\prime} \in \overline{\mathfrak{L}_{0}},
$$

is satisfied, where again $\bar{X}_{4}$ is a $\bar{v}$-measurable subset which differs from $\bar{X}_{3}$ only by a null set.

The $\mathcal{H}_{\xi}$-vectors corresponding to elements of $\overline{\mathfrak{L}_{0}}$ that arise as Poincaré transforms of $L^{\prime}=\alpha_{\left(\Lambda_{0}, x_{0}\right)}^{\bullet}\left(\hat{L}_{0}\right) \in \overline{\mathfrak{L}_{0}}$ are defined according to (4.21), in particular by the relations (4.21e) and (4.21b). As a result, when $\left(\Lambda_{1}, x_{1}\right)$ and $\left(\Lambda_{2}, x_{2}\right)$ are closely neighbouring 
elements of $\mathrm{P}_{+}^{\uparrow}$ so that their products with $\left(\Lambda_{0}, x_{0}\right)$ lie in the common open neighbourhood $\mathrm{V}_{k}$, we get the following estimate, which is a direct consequence of the above constructions inserted into relation (4.20f) and which holds for any $\xi \in \bar{X}_{4}$ :

$$
\begin{aligned}
& \|\left|\alpha_{\left(\Lambda_{1}, x_{1}\right)}^{\bullet}\left(L^{\prime}\right)\right\rangle_{\xi}-\left|\alpha_{\left(\Lambda_{2}, x_{2}\right)}^{\bullet}\left(L^{\prime}\right)\right\rangle_{\xi} \| \\
& \quad \leqslant\left|\phi_{k}\left(\left(\Lambda_{0}, x_{0}\right)\left(\Lambda_{1}, x_{1}\right)\right)-\phi_{k}\left(\left(\Lambda_{0}, x_{0}\right)\left(\Lambda_{2}, x_{2}\right)\right)\right| \cdot D\left(F ; C_{k}\right) \cdot \mu\left(C_{k} \cdot S_{F}\right) \cdot \varphi\left[\hat{L}_{0}, C_{k}\right] \xi\left(\mathbf{1}_{\xi}\right) .
\end{aligned}
$$

This shows that the continuity property with respect to generic Poincare transformations as expressed in (4.8) of Definition 4.1 is fulfilled by all the extended mappings | . $\rangle \xi$ introduced above for arbitrary $L^{\prime} \in \overline{\mathfrak{L}_{0}}$.

(iii) The last property of restricted $\overline{\mathfrak{L}_{0}^{c}}$-particle weights to be established is the existence of unitary groups $\left\{U_{\xi}(x): x \in \mathbb{R}^{s+1}\right\}$ in the representations $\left(\pi_{\xi}, \mathcal{H}_{\xi}\right)$ which satisfy the relations (4.9). To construct them we first consider one element $L$ of the countable space $\mathfrak{L}^{c}$ together with a single space-time translation $y$ in the numerable dense subgroup $\mathrm{T}^{c}$ of $\mathbb{R}^{s+1}$. By assumption (4.11), the von Neumann algebra $\mathfrak{M}$ is contained in the commutant of $\left\{U^{\bullet}(x): x \in \mathbb{R}^{s+1}\right\}$, which means that for any measurable subset $M$ of $\overline{\mathrm{X}}_{4}$ with associated orthogonal projection $P_{M} \in \mathfrak{M}$ there holds the equation

$$
\begin{aligned}
\int_{M} d \bar{v}(\xi) \|\left|\alpha_{y}^{\bullet}(L)\right\rangle_{\xi}\left\|^{2}=\right\| P_{M}\left|\alpha_{y}^{\bullet}(L)\right\rangle^{\bullet}\left\|^{2}=\right\| P_{M} U^{\bullet}(y)|L\rangle^{\bullet} \|^{2} \\
=\| U^{\bullet}(y) P_{M}|L\rangle^{\bullet}\left\|^{2}=\right\| P_{M}|L\rangle^{\bullet}\left\|^{2}=\int_{M} d \bar{v}(\xi)\right\||L\rangle_{\xi} \|^{2} .
\end{aligned}
$$

Since this result is valid for arbitrary measurable sets $M$, we infer by [35, Chapter V, $\S 25$, Theorem E] that for $\bar{v}$-almost all $\xi$ the vectors are subject to the relation

$$
\|\left|\alpha_{y}^{\bullet}(L)\right\rangle_{\xi}\|=\||L\rangle_{\xi} \|
$$

A corresponding equation can be derived for any other of the countable number of combinations of elements in $\mathfrak{L}^{c}$ and $\mathrm{T}^{c}$, so that (4.23b) is true in all of these cases when the domain of $\xi$ is restricted to the $\bar{v}$-measurable subset $\bar{X}_{5}$, which again differs from $\bar{X}_{4}$ only by a null set. On $\bar{X}_{5}$ we can then define for arbitrary $y \in \mathrm{T}^{c}$ the mappings

$$
\bar{U}_{\xi}(y): \mathcal{H}_{\xi}^{c} \rightarrow \mathcal{H}_{\xi}^{c} \quad \bar{U}_{\xi}(y)|L\rangle_{\xi} \doteq\left|\alpha_{y}^{\bullet}(L)\right\rangle_{\xi}
$$

which are indeed determined unambiguously according to (4.23b). By the same relation they are norm-preserving and, moreover, turn out to be $(\mathbb{Q}+i \mathbb{Q})$-linear operators on the countable spaces $\mathcal{H}_{\xi}^{c} \subseteq \mathcal{H}_{\xi}$.

We want to extend the definition given by (4.23c) in two respects: All space-time translations $y \in \mathbb{R}^{s+1}$ should be permissible, and all vectors of $\mathcal{H}_{\xi}$ are to belong to the domain of the resulting operators. Now, let $L$ be an arbitrary element of $\mathfrak{L}^{c}$, i. e.

$$
L=\sum_{i=1}^{N} A_{i} L_{i} \quad \text { with } \quad A_{i} \in \overline{\mathfrak{A}^{c}} \text { and } L_{i} \in \overline{\mathfrak{L}_{0}^{c}},
$$

and consider $x \in \mathbb{R}^{s+1}$ approximated by the sequence $\left\{x_{n}\right\}_{n \in \mathbb{N}} \subseteq \mathrm{T}^{c}$. Then, by definition $(4.23 \mathrm{c})$ in connection with property $(4.15 \mathrm{~b})$, the translates by $x_{k}$ and $x_{l}$ of the 
vectors $|L\rangle_{\xi}$ are for $\xi \in \bar{X}_{5}$ subject to the following relation:

$$
\begin{aligned}
& \bar{U}_{\xi}\left(x_{k}\right)|L\rangle_{\xi}-\bar{U}_{\xi}\left(x_{l}\right)|L\rangle_{\xi}=\left|\alpha_{x_{k}}^{\bullet}\left(\sum_{i=1}^{N} A_{i} L_{i}\right)\right\rangle_{\xi}-\left|\alpha_{x_{l}}^{\bullet}\left(\sum_{i=1}^{N} A_{i} L_{i}\right)\right\rangle_{\xi} \\
& =\sum_{i=1}^{N} \pi_{\xi}\left(\alpha_{x_{k}}^{\bullet}\left(A_{i}\right)\right)\left|\alpha_{x_{k}}^{\bullet}\left(L_{i}\right)\right\rangle_{\xi}-\sum_{i=1}^{N} \pi_{\xi}\left(\alpha_{x_{l}}^{\bullet}\left(A_{i}\right)\right)\left|\alpha_{x_{l}}^{\bullet}\left(L_{i}\right)\right\rangle_{\xi} \\
& =\sum_{i=1}^{N} \pi_{\xi}\left(\alpha_{x_{k}}^{\bullet}\left(A_{i}\right)-\alpha_{x_{l}}^{\bullet}\left(A_{i}\right)\right)\left|\alpha_{x_{k}}^{\bullet}\left(L_{i}\right)\right\rangle_{\xi}+\sum_{i=1}^{N} \pi_{\xi}\left(\alpha_{x_{l}}^{\bullet}\left(A_{i}\right)\right)\left(\left|\alpha_{x_{k}}^{\bullet}\left(L_{i}\right)\right\rangle_{\xi}-\left|\alpha_{x_{l}}^{\bullet}\left(L_{i}\right)\right\rangle_{\xi}\right) .
\end{aligned}
$$

Since $\bar{X}_{3}$ is a subset of $\bar{X}_{5}$, we have relation (4.20f) at our disposal; moreover, by assumption, the group of automorphisms $\left\{\alpha_{(\Lambda, x)}:(\Lambda, x) \in \mathrm{P}_{+}^{\uparrow}\right\}$ is strongly continuous. As a result, the sequences

$$
\left\{\left|\alpha_{x_{k}}^{\bullet}(L)\right\rangle_{\xi}\right\}_{k \in \mathbb{N}}, \quad L \in \overline{\mathfrak{L}_{0}^{c}}, \quad\left\{\pi_{\xi}\left(\alpha_{x_{k}}^{\bullet}(A)\right)\right\}_{k \in \mathbb{N}}, \quad A \in \overline{\mathfrak{A}^{c}},
$$

both have the Cauchy property and are thus convergent as well as bounded in the respective norm topologies. Applied to the elements of $\overline{\mathfrak{L}_{0}^{c}}$ and $\overline{\mathfrak{A}^{c}}$ appearing in the representation (4.24a) of $L \in \mathfrak{L}^{c}$ this has the consequence, that the right-hand side of (4.24b) can be made arbitrarily small for all pairs $k, l \in \mathbb{N}$ exceeding a certain number. The terms on the left-hand side of this inequality thus turn out to be part of Cauchy sequences $\left\{\bar{U}_{\xi}\left(x_{k}\right)|L\rangle_{\xi}\right\}_{k \in \mathbb{N}}$ which converge in the Hilbert spaces $\mathcal{H}_{\xi}$. Since a renewed application of the above arguments shows that the arising limits are independent of the approximating sequence in $\mathrm{T}^{c}$, the following relation unambiguously defines the mappings $\bar{U}_{\xi}(x)$ for arbitrary $x \in \mathbb{R}^{s+1}$ and $L \in \mathfrak{L}^{c}$ :

$$
\begin{gathered}
\bar{U}_{\xi}(x)|L\rangle_{\xi} \doteq \lim _{k \rightarrow \infty} \bar{U}_{\xi}\left(x_{k}\right)|L\rangle_{\xi}=\lim _{k \rightarrow \infty}\left|\alpha_{x_{k}}^{\bullet}(L)\right\rangle_{\xi}, \\
\text { where } \quad \mathrm{T}^{c} \ni x_{k} \underset{k \rightarrow \infty}{\longrightarrow} x \in \mathbb{R}^{s+1} .
\end{gathered}
$$

Again these mappings act as $(\mathbb{Q}+i \mathbb{Q})$-linear operators on the spaces $\mathcal{H}_{\xi}^{c}$ and preserve the Hilbert space norm. As a consequence they can, by the standard procedure used for completions of uniform spaces, be continuously extended in a unique fashion to all of the Hilbert space on condition that their countable domain constitutes a dense subset of $\mathcal{H}_{\xi}$; but this is the case as $\bar{X}_{5}$ is contained in $\bar{X}_{2}$. Changing the notation from $\bar{U}_{\xi}$ to $U_{\xi}$ for these extensions, their definition on arbitrary vectors $\Psi_{\xi} \in \mathcal{H}_{\xi}$ then reads for any $x \in \mathbb{R}^{s+1}$

$$
\begin{gathered}
U_{\xi}(x) \Psi_{\xi} \doteq \lim _{l \rightarrow \infty} \bar{U}_{\xi}(x)\left|L^{(l)}\right\rangle_{\xi}, \\
\text { where } \quad \mathcal{H}_{\xi}^{c} \ni\left|L^{(l)}\right\rangle_{\xi} \underset{l \rightarrow \infty}{\longrightarrow} \Psi_{\xi} \in \mathcal{H}_{\xi},
\end{gathered}
$$

and this definition is again independent of the selected sequence. For any $L \in \mathfrak{L}^{c}$ and any $x \in \mathbb{R}^{s+1}$ the vector field $\left\{U_{\xi}(x)|L\rangle_{\xi}: \xi \in \bar{X}_{5}\right\}$, which is the pointwise limit of a sequence of measurable vector fields by (4.24d) and hence itself measurable [26, Definition II.4.1], corresponds to $\left|\alpha_{x}^{\bullet}(L)\right\rangle^{\bullet}$, the equivalent limit in $\mathcal{H}^{\bullet}$ (where we neglect the 
difference between $\bar{X}$ and $\bar{X}_{5}$ which is of measure 0 ):

$$
\bar{W} U^{\bullet}(x)|L\rangle^{\bullet}=\bar{W}\left|\alpha_{x}^{\bullet}(L)\right\rangle^{\bullet}=\int_{\overline{\mathrm{X}}_{5}}^{\oplus} d \bar{v}(\xi) U_{\xi}(x)|L\rangle_{\xi}
$$

Having defined the family of mappings $\left\{U_{\xi}(x): x \in \mathbb{R}^{s+1}\right\} \subseteq \mathfrak{B}\left(\mathcal{H}_{\xi}\right)$ for $\xi \in \bar{X}_{5}$, we now have to check that they obey (4.9). First of all, note that, as an immediate consequence of the way in which they were introduced, these mappings are $\mathbb{C}$-linear and norm-preserving. Another property that is readily checked by use of the relations (4.24e) and (4.24d) in connection with the estimates arising from (4.24b) with $L^{(l)}$ replacing $L$ is the fact that for arbitrary $x, y \in \mathbb{R}^{s+1}$

$$
U_{\xi}(x) \cdot U_{\xi}(y)=U_{\xi}(x+y) .
$$

From this we infer that, as $U_{\xi}(0)=\mathbf{1}_{\xi}$, each operator $U_{\xi}(x)$ has the inverse $U_{\xi}(-x)$ and thus proves to be an isometric isomorphism of $\mathcal{H}_{\xi}$. Hence, in accordance with (4.25), the set $\left\{U_{\xi}(x): x \in \mathbb{R}^{s+1}\right\}$ indeed turns out to be a unitary group in $\mathfrak{B}\left(\mathcal{H}_{\xi}\right)$.

The strong continuity of this group is easily seen: Consider the operator $L \in \mathfrak{L}^{c}$ as defined in (4.24a) and two sequences $\left\{x_{k}\right\}_{k \in \mathbb{N}},\left\{y_{l}\right\}_{l \in \mathbb{N}}$ in $\mathrm{T}^{c}$ converging to $x$ and $y$, respectively. Then (4.24b) stays valid if we replace the translations $x_{l}$ by $y_{l}$ in each case. In compliance with (4.24d) it is then possible to pass to the limit, which results in the obvious estimate

$$
\begin{aligned}
& \| \bar{U}_{\xi}(x)|L\rangle_{\xi}-\bar{U}_{\xi}(y)|L\rangle_{\xi} \| \\
& \quad \leqslant \sum_{i=1}^{N}\left\|\alpha_{x}^{\bullet}\left(A_{i}\right)-\alpha_{y}^{\bullet}\left(A_{i}\right)\right\| \|\left|\alpha_{x}^{\bullet}\left(L_{i}\right)\right\rangle_{\xi}\left\|+\sum_{i=1}^{N}\right\| A_{i}\|\|\left|\alpha_{x}^{\bullet}\left(L_{i}\right)\right\rangle_{\xi}-\left|\alpha_{y}^{\bullet}\left(L_{i}\right)\right\rangle_{\xi} \| .
\end{aligned}
$$

This explicit inequality shows that the right-hand side can be made arbitrarily small for all $y$ in an appropriate neighbourhood of $x$; for the first term this is brought about by the strong continuity of the automorphism group $\left\{\alpha_{(\Lambda, x)}:(\Lambda, x) \in \mathrm{P}_{+}^{\uparrow}\right\}$, whereas for the second term it is a consequence of relation (4.22). The defining condition for strong continuity is therefore satisfied for vectors in the dense subset $\mathcal{H}_{\xi}^{c}$. If now an arbitrary vector $\Psi_{\xi} \in \mathcal{H}_{\xi}$ is considered, we can expand the difference $U_{\xi}(x) \Psi_{\xi}-U_{\xi}(y) \Psi_{\xi}$ by introducing the corresponding translates of any element $|L\rangle_{\xi} \in \mathcal{H}_{\xi}^{c}$ and, making use of the property of norm-preservation of the unitaries, arrive at

$$
\begin{aligned}
& \left\|U_{\xi}(x) \Psi_{\xi}-U_{\xi}(y) \Psi_{\xi}\right\| \\
& \quad \leqslant \| \Psi_{\xi}-|L\rangle_{\xi}\|+\| \bar{U}_{\xi}(x)|L\rangle_{\xi}-\bar{U}_{\xi}(y)|L\rangle_{\xi}\|+\||L\rangle_{\xi}-\Psi_{\xi} \| .
\end{aligned}
$$

The right-hand side of this inequality can again be made smaller than any given bound by first choosing a suitable element $|L\rangle_{\xi} \in \mathcal{H}_{\xi}^{c}$ from a small neighbourhood of $\Psi_{\xi}$ and then, in dependence on this selected vector $|L\rangle_{\xi}$ but irrelevant for the statement, selecting an appropriate neighbourhood of translations $y$ around $x$ as implied by (4.26a). Thereby we have established strong continuity of the unitary group $\left\{U_{\xi}(x): x \in \mathbb{R}^{s+1}\right\}$.

Before considering the support of the spectral measure $E_{\xi}($.$) associated with this$ strongly continuous unitary group, we mention a result on the interchange of integrations with respect to the Lebesgue measure on $\mathbb{R}^{s+1}$ and the bounded positive measure 
$\bar{v}$ on $\bar{X}_{5}$, which proves to be necessary as Fubini's Theorem does not apply to the situation in question. Let $g$ be a continuous bounded function in $L^{1}\left(\mathbb{R}^{s+1}, d^{s+1} x\right)$, then

$$
\mathbb{R}^{s+1} \ni x \mapsto g(x)\left\langle L_{1}\left|U_{\xi}(x)\right| L_{2}\right\rangle_{\xi} \in \mathbb{C}
$$

is an integrable mapping for any $L_{1}, L_{2} \in \mathfrak{L}^{c}$ and $\xi \in \bar{X}_{5}$. Moreover, it is Riemann integrable over any compact $(s+1)$-dimensional interval $\boldsymbol{K}$, and this integral is the limit of a Riemann sequence (cf. [37, Kapitel XXIII, Abschnitt 197 and Lebesguesches Integrabilitätskriterium 199.3]):

$$
\int_{\boldsymbol{K}} d^{s+1} x g(x)\left\langle L_{1}\left|U_{\xi}(x)\right| L_{2}\right\rangle_{\xi}=\lim _{i \rightarrow \infty} \sum_{m=1}^{n_{i}}\left|Z_{m}^{(i)}\right| g\left(x_{m}^{(i)}\right)\left\langle L_{1}\left|U_{\xi}\left(x_{m}^{(i)}\right)\right| L_{2}\right\rangle_{\xi},
$$

where $\left\{Z_{m}^{(i)}: m=1, \ldots, n_{i}\right\}$ denotes the $i$-th subdivision of $\boldsymbol{K},\left|Z_{m}^{(i)}\right|$ are the Lebesgue measures of these sets, and $x_{m}^{(i)} \in Z_{m}^{(i)}$ are corresponding intermediate points. The sums on the right-hand side of this equation turn out to be $\bar{v}$-measurable when their dependence on $\xi$ is taken into account, and so is the limit on the left-hand side. Moreover this property is preserved in passing to the limit $\boldsymbol{\nearrow} \mathbb{R}^{s+1}$, so that

$$
\bar{X}_{5} \ni \xi \mapsto \int_{\mathbb{R}^{s+1}} d^{s+1} x g(x)\left\langle L_{1}\left|U_{\xi}(x)\right| L_{2}\right\rangle_{\xi} \in \mathbb{C}
$$

is $\bar{v}$-measurable and, in addition, integrable since

$$
\begin{aligned}
\int_{\overline{\mathrm{X}}_{5}} d \bar{v}(\xi)\left|\int_{\mathbb{R}^{s+1}} d^{s+1} x g(x)\left\langle L_{1}\left|U_{\xi}(x)\right| L_{2}\right\rangle_{\xi}\right| \\
\leqslant \int_{\bar{X}_{5}} d \bar{v}(\xi) \int_{\mathbb{R}^{s+1}} d^{s+1} x|g(x)| \|\left|L_{1}\right\rangle_{\xi}\|\|\left|L_{2}\right\rangle_{\xi} \| \\
\quad=\|g\|_{1} \int_{\bar{X}_{5}} d \bar{v}(\xi) \|\left|L_{1}\right\rangle_{\xi}\|\|\left|L_{2}\right\rangle_{\xi}\|\leqslant\| g\left\|_{1}\right\|\left|L_{1}\right\rangle^{\bullet}\|\|\left|L_{2}\right\rangle^{\bullet} \| .
\end{aligned}
$$

The counterpart of (4.27a) is valid in $\mathcal{H}^{\bullet}$, too, and, if $M$ denotes a measurable subset of $\bar{X}_{5}$ with associated orthogonal projection $P_{M} \in \mathfrak{M}$, this equation reads

$$
\int_{\boldsymbol{K}} d^{S+1} x g(x)\left\langle L_{1}\left|P_{M} U^{\bullet}(x)\right| L_{2}\right\rangle=\lim _{i \rightarrow \infty} \sum_{m=1}^{n_{i}}\left|z_{m}^{(i)}\right| g\left(x_{m}^{(i)}\right)\left\langle L_{1}\left|P_{M} U^{\bullet}\left(x_{m}^{(i)}\right)\right| L_{2}\right\rangle .
$$

Then (4.16) and (4.24f) in connection with (4.14c) imply

$$
\begin{aligned}
\int_{\boldsymbol{K}} d^{s+1} x g(x)\left\langle L_{1}\left|P_{M} U^{\bullet}(x)\right| L_{2}\right\rangle & =\lim _{i \rightarrow \infty} \int_{M} d \bar{v}(\xi) \sum_{m=1}^{n_{i}}\left|Z_{m}^{(i)}\right| g\left(x_{m}^{(i)}\right)\left\langle L_{1}\left|U_{\xi}\left(x_{m}^{(i)}\right)\right| L_{2}\right\rangle_{\xi} \\
& =\int_{M} d \bar{\nu}(\xi) \int_{\boldsymbol{K}} d^{s+1} x g(x)\left\langle L_{1}\left|U_{\xi}(x)\right| L_{2}\right\rangle_{\xi}, \quad(4.27 \mathrm{~d})
\end{aligned}
$$

where, in the second equation, use was made of Lebesgue's Dominated Convergence Theorem in view of the fact that the integrable function

$$
\bar{X}_{5} \ni \xi \mapsto\|g\|_{1} \|\left|L_{1}\right\rangle_{\xi}\|\|\left|L_{2}\right\rangle \xi \| \in \mathbb{C}
$$


majorizes both sides of (4.27a). Relation (4.27d) again stays true in passing to the limit K $\nearrow \mathbb{R}^{s+1}$ :

$$
\int_{\mathbb{R}^{s+1}} d^{s+1} x g(x)\left\langle L_{1}\left|P_{M} U^{\bullet}(x)\right| L_{2}\right\rangle=\int_{M} d \bar{\nu}(\xi) \int_{\mathbb{R}^{s+1}} d^{s+1} x g(x)\left\langle L_{1}\left|U_{\xi}(x)\right| L_{2}\right\rangle_{\xi},
$$

which is the announced result on the commutability of integrations in the present context.

The support of the spectral measure $E_{\xi}($.$) associated with the generators P_{\xi}$ of the unitary group $\left\{U_{\xi}(x): x \in \mathbb{R}^{s+1}\right\}$ can now be investigated by use of the method applied in the proof of the fourth item of the first part of Theorem 3.15. Note, that the complement of the closed set $\bar{V}_{+}-q \subseteq \mathbb{R}^{s+1}$ can be covered by an increasing sequence $\left\{\Gamma_{N}\right\}_{N \in \mathbb{N}}$ of compact subsets (take e. g. the intersection of the compact ball of radius $N$ with the complement of the open $N^{-1}$-neighbourhood of $\bar{V}_{+}-q$ ). To each of these sets one can find an infinitely often differentiable function $\tilde{g}_{N}$ with support in $\complement\left(\bar{V}_{+}-q\right)$ that has the property $0 \leqslant \chi_{\Gamma_{N}} \leqslant \tilde{g}_{N}$. As before, let $M$ be a measurable subset of $\bar{X}_{5}$ with associated orthogonal projection $P_{M} \in \mathfrak{M}$, then, by assumption on the spectral support of the unitary group implementing space-time translations in the underlying particle weight, we have

$$
\int_{\mathbb{R}^{s+1}} d^{s+1} x g_{N}(x)\left\langle L_{1}\left|P_{M} U^{\bullet}(x)\right| L_{2}\right\rangle=0
$$

for any $N \in \mathbb{N}$ and any pair of vectors $\left|L_{1}\right\rangle$ and $\left|L_{2}\right\rangle$ with $L_{1}, L_{2} \in \mathfrak{L}^{c}$, and this, according to $(4.27 \mathrm{e})$, implies

$$
\int_{M} d \bar{v}(\xi) \int_{\mathbb{R}^{s+1}} d^{s+1} x g_{N}(x)\left\langle L_{1}\left|U_{\xi}(x)\right| L_{2}\right\rangle_{\xi}=0 .
$$

By arbitrariness of $M$ in the last expression, we conclude once more that for $\bar{v}$-almost all $\xi \in \bar{X}_{5}$

$$
\int_{\mathbb{R}^{s+1}} d^{s+1} x g_{N}(x)\left\langle L_{1}\left|U_{\xi}(x)\right| L_{2}\right\rangle_{\xi}=0
$$

Eventually, if we want this equation to hold for any element of the countable set of triples $\left(g_{N},\left|L_{1}\right\rangle_{\xi},\left|L_{2}\right\rangle_{\xi}\right)$, a non-null set $\overline{\mathrm{X}}_{6} \subseteq \overline{\mathrm{X}}_{5}$ is left, and (4.28c) stays valid for the remaining $\xi \in \bar{X}_{6}$ even if the special vectors $\left|L_{1}\right\rangle_{\xi}$ and $\left|L_{2}\right\rangle_{\xi}$ are replaced by arbitrary ones. Stone's Theorem then implies (cf. (3.38)) that $\tilde{g}_{N}\left(P_{\xi}\right)=0$ and therefore, by the order relation inherent in the definition of $\tilde{g}_{N}$, we have $E_{\xi}\left(\Gamma_{N}\right)=\chi_{\Gamma_{N}}\left(P_{\xi}\right)=0$ for any $N \in \mathbb{N}$. As the spectral measure $E_{\xi}($.$) is regular, one can pass to the limit N \rightarrow \infty$ and thereby arrives at the desired result

$$
E_{\xi}\left(\complement\left(\bar{V}_{+}-q\right)\right)=0, \quad \xi \in \bar{X}_{6} .
$$

The defining equation (4.23c) in connection with (4.15b) implies that for arbitrary operators $A^{\prime} \in \overline{\mathfrak{A}^{c}}$ and $L \in \mathfrak{L}^{c}$ and for any translation $x^{\prime} \in \mathrm{T}^{c}$ one can write

$$
\begin{aligned}
\pi_{\xi}\left(\alpha_{x^{\prime}}^{\bullet}\left(A^{\prime}\right)\right)|L\rangle_{\xi}= & \left|\alpha_{x^{\prime}}^{\bullet}\left(A^{\prime}\right) L\right\rangle_{\xi}=\left|\alpha_{x^{\prime}}^{\bullet}\left(A^{\prime} \alpha_{-x^{\prime}}^{\bullet}(L)\right)\right\rangle_{\xi}=\bar{U}_{\xi}\left(x^{\prime}\right)\left|A^{\prime} \alpha_{-x^{\prime}}^{\bullet}(L)\right\rangle_{\xi} \\
& =\bar{U}_{\xi}\left(x^{\prime}\right) \pi_{\xi}\left(A^{\prime}\right) \bar{U}_{\xi}\left(-x^{\prime}\right)|L\rangle_{\xi}=\bar{U}_{\xi}\left(x^{\prime}\right) \pi_{\xi}\left(A^{\prime}\right) \bar{U}_{\xi}\left(x^{\prime}\right)^{*}|L\rangle_{\xi}
\end{aligned}
$$


Since the vectors $|L\rangle_{\xi} \in \mathcal{H}_{\xi}^{c}, L \in \mathfrak{L}^{c}$, constitute a dense subset of $\mathcal{H}_{\xi}$ for $\xi \in \bar{X}_{2} \subseteq \bar{X}_{5}$ we infer from this equation that

$$
\pi_{\xi}\left(\alpha_{x^{\prime}}^{\bullet}\left(A^{\prime}\right)\right)=\bar{U}_{\xi}\left(x^{\prime}\right) \pi_{\xi}\left(A^{\prime}\right) \bar{U}_{\xi}\left(x^{\prime}\right)^{*},
$$

an equation that readily extends to all translations $x$ in $\mathbb{R}^{s+1}$ and, by uniform density of $\overline{\mathfrak{A}^{c}}$ in $\mathfrak{A}^{\bullet}$, also to any operator $A$ in the $C^{*}$-algebra $\mathfrak{A}^{\bullet}$ :

$$
\pi_{\xi}\left(\alpha_{x}^{\bullet}(A)\right)=U_{\xi}(x) \pi_{\xi}(A) U_{\xi}(x)^{*}, \quad A \in \mathfrak{A}^{\bullet}, \quad x \in \mathbb{R}^{s+1} .
$$

This proves the counterpart of (4.9a). The action of the group $\left\{U_{\xi}(x): x \in \mathbb{R}^{s+1}\right\}$ on $\left\{\left|L^{\prime}\right\rangle_{\xi}: L^{\prime} \in \overline{\mathfrak{L}_{0}}\right\}$ according to (4.9b) is an immediate consequence of the defining relations $(4.24 \mathrm{~d})$ and $(4.24 \mathrm{e})$ in connection with (4.21e) and the continuity property as expressed by (4.22). In the present setting we thus have

$$
U_{\xi}(x)\left|L^{\prime}\right\rangle_{\xi} \doteq\left|\alpha_{x}^{\bullet}\left(L^{\prime}\right)\right\rangle_{\xi}, \quad L^{\prime} \in \overline{\mathfrak{L}_{0}} .
$$

Now, let $L$ be an arbitrary element of $\mathfrak{L}^{c}$ having energy-momentum transfer $\Gamma_{L}$. Defined as the support of the Fourier transform of an operator-valued distribution (cf. the Remark following Definition 2.2), $\Gamma_{L}$ is a closed Borel set, so that the arguments given in the preceding paragraph can again be applied when $L$ replaces $L_{1}$ and $L_{2}$ and the functions $\tilde{g}_{N}$ now correspond to an increasing sequence of compact sets $\Gamma_{N}^{\prime}$ which constitute a cover of $\complement \Gamma_{L}$. As a result we arrive at the equivalent of (4.28c), so that

$$
\int_{\mathbb{R}^{s+1}} d^{s+1} x g_{N}(x)\left\langle L\left|U_{\xi}(x)\right| L\right\rangle_{\xi}=0
$$

holds for $\bar{v}$-almost all $\xi \in \overline{\mathrm{X}}_{6}$ even if the index $N$ is allowed to run through all natural numbers. As in the preceding paragraph we then conclude that for all of these $\xi$ and all $N \in \mathbb{N}$ one has $\left\langle L\left|\tilde{g}_{N}\left(P_{\xi}\right)\right| L\right\rangle_{\xi}=0$ and hence $\left\langle L\left|E_{\xi}\left(\Gamma_{N}^{\prime}\right)\right| L\right\rangle_{\xi}=0$. According to the regularity of the spectral measure $E_{\xi}($.$) , passage to the limit with respect to N$ yields the equation $E_{\xi}\left(C \Gamma_{L}\right)|L\rangle_{\xi}=0$. By countability, this last result is valid for arbitrary $L \in \mathfrak{L}^{c}$ if a $\bar{v}$-measurable non-null set $\bar{X}_{7} \subseteq \bar{X}_{6}$ is appropriately selected, from which the indices $\xi$ are to be taken. The complementary statement thus constitutes a restricted version of the counterpart of $(4.9 \mathrm{c})$ :

$$
E_{\xi}\left(\Gamma_{L}\right)|L\rangle_{\xi}=|L\rangle_{\xi}, \quad L \in \mathfrak{L}^{c}, \quad \xi \in \bar{X}_{7}
$$

Now, let $\hat{L}_{0}$ be an arbitrary element of $\overline{\mathfrak{L}_{0}^{c}}$, then the energy-momentum transfer of its Poincaré transform by $(\Lambda, x) \in \mathrm{P}^{c}$, i. e. of the operator $\alpha_{(\Lambda, x)}^{\bullet}\left(\hat{L}_{0}\right) \in \overline{\mathfrak{L}_{0}^{c}} \subseteq \mathfrak{L}^{c}$, is given by $\Lambda \Gamma_{\hat{L}_{0}}$, so that, according to (4.31b),

$$
E_{\xi}\left(\Lambda \Gamma_{\hat{L}_{0}}\right)\left|\alpha_{(\Lambda, x)}^{\bullet}\left(\hat{L}_{0}\right)\right\rangle_{\xi}=\left|\alpha_{(\Lambda, x)}^{\bullet}\left(\hat{L}_{0}\right)\right\rangle_{\xi}, \quad \xi \in \bar{X}_{7}
$$

This result can be applied to investigate the case of a generic element from $\overline{\mathfrak{L}_{0}}$. For arbitrary $\left(\Lambda_{0}, x_{0}\right) \in \mathrm{P}_{+}^{\uparrow}$ approximated by the sequence $\left\{\left(\Lambda_{n}, x_{n}\right)\right\}_{n \in \mathbb{N}} \subseteq \mathrm{P}^{c}$ we have, by virtue of the relevant parts of (4.21),

$$
\left|\alpha_{\left(\Lambda_{0}, x_{0}\right)}^{\bullet}\left(\hat{L}_{0}\right)\right\rangle_{\xi}=\lim _{n \rightarrow \infty}\left|\alpha_{\left(\Lambda_{n}, x_{n}\right)}^{\bullet}\left(\hat{L}_{0}\right)\right\rangle_{\xi},
$$


and Lebesgue's Dominated Convergence Theorem in connection with Stone's Theorem yields for any function $g \in L^{1}\left(\mathbb{R}^{s+1}, d^{s+1} x\right)$ and any index $\xi \in \bar{X}_{7}$

$$
\begin{array}{rl}
\int_{\mathbb{R}^{s+1}} d^{s+1} x & g(x)\left\langle\alpha_{\left(\Lambda_{0}, x_{0}\right)}^{\bullet}\left(\hat{L}_{0}\right)\left|U_{\xi}(x)\right| \alpha_{\left(\Lambda_{0}, x_{0}\right)}^{\bullet}\left(\hat{L}_{0}\right)\right\rangle_{\xi} \\
=\lim _{n \rightarrow \infty} \int_{\mathbb{R}^{s+1}} d^{s+1} x g(x)\left\langle\alpha_{\left(\Lambda_{n}, x_{n}\right)}^{\bullet}\left(\hat{L}_{0}\right)\left|U_{\xi}(x)\right| \alpha_{\left(\Lambda_{n}, x_{n}\right)}^{\bullet}\left(\hat{L}_{0}\right)\right\rangle_{\xi} \\
\quad=(2 \pi)^{(s+1) / 2} \lim _{n \rightarrow \infty}\left\langle\alpha_{\left(\Lambda_{n}, x_{n}\right)}^{\bullet}\left(\hat{L}_{0}\right)\left|\tilde{g}\left(P_{\xi}\right)\right| \alpha_{\left(\Lambda_{n}, x_{n}\right)}^{\bullet}\left(\hat{L}_{0}\right)\right\rangle_{\xi}
\end{array}
$$

In the limit of large $n$ one finds the energy-momentum transfer $\Lambda_{n} \Gamma_{\hat{L}_{0}}$ of $\alpha_{\left(\Lambda_{n}, x_{n}\right)}\left(\hat{L}_{0}\right)$ in a small $\varepsilon$-neighbourhood of $\Lambda_{0} \Gamma_{\hat{L}_{0}}$. Therefore, in view of (4.31c), the right-hand side of (4.31d) vanishes for all $n$ exceeding a certain bound $N$ if $g$ is chosen in such a way that supp $\tilde{g} \subseteq \complement\left(\Lambda_{0} \Gamma_{\hat{L}_{0}}\right)$. The Fourier transform of the distribution

$$
\mathbb{R}^{s+1} \ni x \mapsto\left\langle\alpha_{\left(\Lambda_{0}, x_{0}\right)}^{\bullet}\left(\hat{L}_{0}\right)\left|U_{\xi}(x)\right| \alpha_{\left(\Lambda_{0}, x_{0}\right)}^{\bullet}\left(\hat{L}_{0}\right)\right\rangle_{\xi} \in \mathbb{C}
$$

is thus seen to be supported by $\Lambda_{0} \Gamma_{\hat{L}_{0}}$ from which we infer

$$
E_{\xi}\left(\Lambda_{0} \Gamma_{\hat{L}_{0}}\right)\left|\alpha_{\left(\Lambda_{0}, x_{0}\right)}^{\bullet}\left(\hat{L}_{0}\right)\right\rangle_{\xi}=\left|\alpha_{\left(\Lambda_{0}, x_{0}\right)}^{\bullet}\left(\hat{L}_{0}\right)\right\rangle_{\xi}, \quad \xi \in \bar{X}_{7}
$$

which is the equivalent of (4.31c) for arbitrary operators in $\overline{\mathfrak{L}_{0}}$. Equations (4.31b) and (4.31e) are readily generalized, making use of the order structure of spectral projections reflecting the inclusion relation of Borel subsets of $\mathbb{R}^{s+1}$. If $\overline{\mathfrak{L}}\left(\Delta^{\prime}\right)$ denotes the set of operators from $\mathfrak{L}^{c} \cup \overline{\mathfrak{L}_{0}^{c}}$ having energy-momentum transfer in the Borel set $\Delta^{\prime}$, then

$$
E_{\xi}\left(\Delta^{\prime}\right)|L\rangle_{\xi}=|L\rangle_{\xi}, \quad L \in \overline{\mathfrak{L}}\left(\Delta^{\prime}\right),
$$

and thus the counterpart of (4.9c) is established for the remaining indices $\xi$ from the non-null subset $\bar{X}_{7}$ of $\bar{X}$.

The above construction has supplied us with a measurable subset $X \doteq \bar{X}_{7}$ of the standard Borel space $\bar{X}$, that was introduced at the outset, emerging from an application of [25, Theorem 8.5.2]. $X$ is a non-null set, differing from $\bar{X}$ only by a $\bar{v}$-null set. Moreover it is itself a standard Borel space (cf. the definition in [4, Section 3.3]), and we shall denote the restriction of the measure $\bar{v}$ to it by $v ; v \doteq \bar{v}\lceil X$ is again a bounded positive measure. Moreover, and this has been the central aim of the previous investigations, the field

$$
X \ni \xi \mapsto\left(\pi_{\xi}, \mathcal{H}_{\xi}, \mathfrak{A}^{\bullet}, \alpha^{\bullet}, \mathrm{P}^{c}, \overline{\mathfrak{L}_{0}^{c}}\right),
$$

indeed consists of $\overline{\mathfrak{L}_{0}^{c}}$-particle weights. What remains to be done now is a verification of the properties listed in (4.13).

(i) Arising as the restriction to a measurable subset in $\bar{X}$ of a field of irreducible representations, the family of representations

$$
X \ni \xi \mapsto\left(\pi_{\xi}, \mathcal{H}_{\xi}\right)
$$

is obviously $v$-measurable and its components inherit the feature of irreducibility. 
(ii) As $\mathrm{X}$ and $\overline{\mathrm{X}}$ only differ by a $\overline{\mathrm{V}}$-null set, one has

$$
\int_{\overline{\mathrm{X}}}^{\oplus} d \bar{v}(\xi) \mathcal{H}_{\xi} \simeq \int_{\mathrm{X}}^{\oplus} d v \mathcal{H}_{\xi},
$$

and the relations (4.14) can be reformulated, using the right-hand side of (4.32) and an isomorphism $W$, which is the composition of $\bar{W}$ with the isometry that implements the above unitary equivalence. As an immediate consequence of (4.14a) and (4.14b) we then get the equivalence assertion of (4.13a). Moreover, by (4.16) and (4.21f), the operator $W$ connects the vector fields $\left\{|L\rangle_{\xi}: \xi \in X\right\}$ with vectors $|L\rangle^{\bullet}$ for $L \in \mathfrak{L}^{c} \cup \overline{\mathfrak{L}_{0}}$ in the desired way as expressed in (4.13b).

(iii) (4.13c) is the mere reformulation of (4.14c) in terms of $X$ and $W$.

(iv) According to the argument preceding (4.24f), the mappings $\xi \mapsto\left\langle L_{1}\left|U_{\xi}(x)\right| L_{2}\right\rangle_{\xi}$, with $\xi$ restricted to $X$ and $L_{1}$ as well as $L_{2}$ taken from $\mathfrak{L}^{c}$, are measurable for all vectors $\left|L_{1}\right\rangle_{\xi}$ and $\left|L_{2}\right\rangle_{\xi}$ in the dense subsets $\mathcal{H}_{\xi}^{c}$, and this suffices, by [24, Section II.2.1, Proposition 1], to establish measurability of the field $\xi \mapsto U_{\xi}(x)$ for arbitrary $x \in \mathbb{R}^{s+1}$. Moreover, this is a bounded field of operators, so that it defines a bounded operator on $\mathcal{H}^{\bullet}$ which is given by (4.13d) as an immediate consequence of (4.24f), bearing in mind that $X$ and $\bar{X}_{5}$ only differ by a $\bar{v}$-null set. The demonstration of (4.13e) on the other hand is less straightforward. Assume first of all that the Borel set $\Delta$ in question is open. Then we can make use of the regularity of spectral measures and construct, according to [26, Definition II.8.2], a sequence of compact subsets $\left\{\Gamma_{N}\right\}_{N \in \mathbb{N}}$ as well as of infinitely often differentiable functions $\left\{\tilde{g}_{N}\right\}_{N \in \mathbb{N}}$ with support in $\Delta$ such that $0 \leqslant \chi_{\Gamma_{N}} \leqslant \tilde{g}_{N} \leqslant \chi_{\Delta}$ and furthermore

$$
\begin{gathered}
\left\langle L\left|E_{\xi}(\Delta)\right| L\right\rangle_{\xi}=\lim _{N \rightarrow \infty}\left\langle L\left|E_{\xi}\left(\Gamma_{N}\right)\right| L\right\rangle_{\xi}=\lim _{N \rightarrow \infty}\left\langle L\left|\tilde{g}_{N}\left(P_{\xi}\right)\right| L\right\rangle_{\xi}, \\
\left\langle L\left|E^{\bullet}(\Delta)\right| L\right\rangle=\lim _{N \rightarrow \infty}\left\langle L\left|E^{\bullet}\left(\Gamma_{N}\right)\right| L\right\rangle=\lim _{N \rightarrow \infty}\left\langle L\left|\tilde{g}_{N}\left(P^{\bullet}\right)\right| L\right\rangle
\end{gathered}
$$

for any $L \in \mathfrak{L}^{c}$. The discussion on page $73 \mathrm{f}$.- -with $X$ replacing $\bar{X}_{5}$ and $v$ instead of $\bar{v}$-demonstrates, making use of Stone's Theorem, that the sequence appearing on the right-hand side consists of $v$-measurable functions of $\xi$, so that we infer that its limit

$$
\mathrm{X} \ni \xi \mapsto\left\langle L\left|E_{\xi}(\Delta)\right| L\right\rangle_{\xi} \in \mathbb{C}
$$

is $v$-measurable, too. Another application of Stone's Theorem in connection with (4.27e) in terms of $X$ then shows that

$$
\begin{aligned}
& (2 \pi)^{(s+1) / 2}\left\langle L\left|\tilde{g}_{N}\left(P^{\bullet}\right)\right| L\right\rangle=\int_{\mathbb{R}^{s+1}} d^{s+1} x g_{N}(x)\left\langle L\left|U^{\bullet}(x)\right| L\right\rangle \\
& \quad=\int_{\mathrm{X}} d \bar{v}(\xi) \int_{\mathbb{R}^{s+1}} d^{s+1} x g_{N}(x)\left\langle L\left|U_{\xi}(x)\right| L\right\rangle_{\xi}=(2 \pi)^{(s+1) / 2} \int_{\mathrm{X}} d \bar{v}(\xi)\left\langle L\left|\tilde{g}_{N}\left(P_{\xi}\right)\right| L\right\rangle_{\xi},
\end{aligned}
$$

and, as Lebesgue's Dominated Convergence Theorem allows for the passage to the limit function under the last integral, we get according to (4.33a) and (4.33b)

$$
\left\langle L\left|E^{\bullet}(\Delta)\right| L\right\rangle=\int_{\mathrm{X}} d \bar{v}(\xi)\left\langle L\left|E_{\xi}(\Delta)\right| L\right\rangle_{\xi}
$$


This formula, as yet valid only for open Borel sets $\Delta$, is readily generalized to closed Borel sets and then, since by regularity the spectral measure of an arbitrary Borel set is approximated by a sequence in terms of compact subsets of it, to any Borel set. By polarization and the fact that the ket vectors with entries from $\mathfrak{L}^{c}$ are dense in $\mathcal{H}^{\bullet}$ and $\mathcal{H}_{\xi}$, respectively, we first conclude with [24, Section II.2.1, Proposition 1] that all the fields $\xi \mapsto E_{\xi}(\Delta)$ are measurable for arbitrary Borel sets $\Delta$ and then pass to the aspired formula (4.13e) from (4.33d).

(v) The unitary operators $V^{\bullet}(x), x \in \mathbb{R}^{s+1}$, defined in (4.10b) belong to the von Neumann algebra $\mathfrak{M}$, according to (4.11), and are thus diagonalisable in the form

$$
W V^{\bullet}(x) W^{*}=\int_{\mathrm{X}}^{\oplus} d v(\xi) \exp \left(i p_{\xi} x\right) \mathbf{1}_{\xi}
$$

According to the construction of these operators, we can re-express this result in terms of the canonical unitary group of (4.10a):

$$
W U_{c}^{\bullet}(x) W^{*}=\int_{X}^{\oplus} d v(\xi) \exp \left(i p_{\xi} x\right) U_{\xi}(x) .
$$

The definition

$$
U_{\xi}^{c}(x) \doteq \exp \left(i p_{\xi} x\right) U_{\xi}(x), \quad x \in \mathbb{R}^{s+1}, \quad \xi \in X,
$$

then provides the asserted canonical choice of a strongly continuous unitary group on each Hilbert space $\mathcal{H}_{\xi}$. Its spectral properties are derived from those of the canonical group $\left\{U_{c}^{\bullet}(x): x \in \mathbb{R}^{s+1}\right\}$ by the methods that have already been used repeatedly above. Possibly a further $v$-null subset of $X$ gets lost by this procedure.

This finishes the proof of the assertions of Theorem 4.4.

Remark. Theorem 4.4 includes the existence of a spatial disintegration of the strongly continuous unitary group implementing space-time translations in the representation $\left(\pi^{\bullet}, \mathcal{H}^{\bullet}\right)$ as well as of the spectral measure associated with it. The method used in the demonstration of this fact can be generalized to other symmetry groups; however obvious a problem of this kind may seem in the present context, it has, to our knowledge, not been treated in the literature. Nevertheless, the disintegration of unbounded closed operators in Hilbert spaces (the self-adjoint generators of strongly continuous unitary groups being an example) is the topic of [46] and also presented in [52, Chapter 12].

At present we have no control over the range of energy-momenta $p_{\xi}$ which enter into the above disintegration theory. It still has to be investigated if, starting from a physical state of bounded energy in the constructions of Chapter 3 and passing to the asymptotic limit with respect to a function $h$ that has support on a small part of the velocity domain, the occurring momenta are correlated with those defined by the geometric momenta involved in the limiting procedure. Even tachyonic states cannot be ruled out to date. These problems might be tackled by introducing a certain property of "closability' for particle weights, stating that, in case that a sequence of operators $\left\{L_{n}\right\}_{n \in \mathbb{N}}$ approaches 0 in a suitable topology and at the same time $\left\{\left|L_{n}\right\rangle\right\}_{n \in \mathbb{N}}$ is convergent, the limit of the sequence of vectors likewise vanishes. 
Moreover, the spatial disintegration presented above is subject to arbitrariness in two respects. There exist different constructions of the type expounded in Section 4.1 and therefore, according to Theorem 4.3, one has to deal with a number of different restricted $\overline{\mathfrak{L}_{0}^{c}}$-particle weights $\left(\pi^{\bullet}, \mathcal{H}^{\bullet}, \mathfrak{A}^{\bullet}, \alpha^{\bullet}, \mathrm{P}^{c}, \overline{\mathfrak{L}_{0}^{c}}\right)$ derived from the GNS-representation $\left(\pi_{w}, \mathcal{H}_{w}\right)$ pertaining to a given particle weight. As a result, the object to be disintegrated according to Theorem 4.4 is by no means uniquely fixed. And even if one has decided to select a system complying with the requirements of this theorem, there still remains an ambiguity as to the choice of a maximal abelian von Neumann algebra, with respect to which the disintegration is to be performed. The same problem is encountered in the framework of Choquet disintegration theory in its present status (cf. the end of Chapter 6). There a suitable base in the positive cone of particle weights has to be chosen with respect to which the disintegration is to be carried through.

But it should be stressed that these open questions only arise on the basis of the fact that a disintegration of general particle weights into pure ones, representing elementary systems, has successfully been constructed. 


\section{Chapter 5}

\section{Phase Space Restrictions and Local Normality}

A number of criteria have been introduced into the analysis of generic quantum field theories in order to dismiss those which are not reasonable form a physical point of view in that they do not allow for an interpretation in terms of particles. These attempts can be traced back to the year 1965 when Haag and Swieca proposed a compactness condition in [34], imposing an effective restriction to the size of phase space. Subsequently, the notion of nuclearity has entered the stage, determining maximum values for the number of local degrees of freedom for physical states of bounded energy (cf. the discussions of [20,16, 31], and in addition [18] for a treatment of the interdependence of these various concepts). In the present context we want to make use of the compactness condition proposed by Fredenhagen and Hertel [28] to show that, under this physically motivated presupposition, the arbitrariness in the choice of a separable $C^{*}$-subalgebra $\mathfrak{A}^{\bullet}$ of the quasi-local algebra $\mathfrak{A}$ in Chapter 4 can be removed.

\section{Compactness Criterion (Fredenhagen-Hertel).}

A local quantum field theory, as introduced in Chapter 1, qualifies the FredenhagenHertel Compactness Condition if the mappings $T_{\Delta^{\prime}}^{\mathcal{O}}$, which are defined for any bounded Borel set $\Delta^{\prime} \subseteq \mathbb{R}^{s+1}$ and any bounded region $\mathcal{O}$ of Minkowski space through

$$
T_{\Delta^{\prime}}^{\mathcal{O}}: \mathfrak{A}(\mathcal{O}) \rightarrow \mathfrak{B}(\mathcal{H}) \quad A \mapsto T_{\Delta^{\prime}}^{\mathcal{O}}(A) \doteq E\left(\Delta^{\prime}\right) A E\left(\Delta^{\prime}\right),
$$

send bounded subsets of $\mathfrak{A}(\mathcal{O})$ onto precompact subsets of $\mathfrak{B}(\mathcal{H})$ with respect to its uniform topology. Precompactness is synonymous with totally boundedness and, in the present situation, equivalent to relative compactness [44, Chapter One, § 4, 5.].

To be able to demonstrate the main result of this chapter, Theorem 5.3, we have to fall back upon $\Delta$-bounded particle weights as introduced in Definition 3.19. This restriction can be motivated on physical grounds, as opposed to mere technical needs, since, according to Lemma 3.20, the asymptotic functionals in $\mathfrak{C}_{\Delta}{ }^{*+}$, constructed by use of physical states of bounded energy in Chapter 3, give rise to particle weights of this special kind. The corresponding GNS-representations $\left(\pi_{w}, \mathcal{H}_{w}\right)$ then meet the Fredenhagen-Hertel Compactness Condition if the underlying local quantum field theory does, and the same holds true for the restricted $\overline{\mathfrak{L}_{0}^{c}}$-particle weights which can be derived from them as expounded in Chapter 4. 
Proposition 5.1. Suppose that the given local quantum field theory satisfies the Compactness Criterion of Fredenhagen and Hertel.

(i) If $\langle. \mid$.$\rangle is a \Delta$-bounded particle weight on $\mathfrak{L} \times \mathfrak{L}$, then the associated GNSrepresentation $\left(\pi_{w}, \mathcal{H}_{w}\right)$ of the quasi-local algebra $\mathfrak{A}$ is subject to the compactness condition as well.

(ii) The restricted $\overline{\mathfrak{L}_{0}^{c}}$-particle weight $\left(\pi^{\bullet}, \mathcal{H}^{\bullet}, \mathfrak{A}^{\bullet}, \alpha^{\bullet}, \mathrm{P}^{c}, \overline{\mathfrak{L}_{0}^{c}}\right)$ derived from the above GNS-representation by virtue of Theorem 4.3 likewise inherits the compactness property in question.

Proof. (i) $\Delta$-boundedness of the particle weight $\langle. \mid$.$\rangle means, according to Defin-$ ition 3.19, that to any bounded Borel set $\Delta^{\prime} \subseteq \mathbb{R}^{s+1}$ there exist another such set $\bar{\Delta}$ containing $\Delta+\Delta^{\prime}$ and an appropriate positive constant $c$, so that the estimate

$$
\left\|E_{w}\left(\Delta^{\prime}\right) \pi_{w}(A) E_{w}\left(\Delta^{\prime}\right)\right\| \leqslant c \cdot\|E(\bar{\Delta}) A E(\bar{\Delta})\|
$$

holds for any $A \in \mathfrak{A}$. Then a finite cover of $T_{\bar{\Delta}}^{\mathcal{O}}\left(\mathfrak{A}_{r}(\mathcal{O})\right)=E(\bar{\Delta}) \mathfrak{A}_{r}(\mathcal{O}) E(\bar{\Delta})$ by sets of diameter less than a given $\delta>0$ (which exists on account of the hypothesis of precompactness) induces a corresponding cover of $E_{w}\left(\Delta^{\prime}\right) \pi_{w}\left(\mathfrak{A}_{r}(\mathcal{O})\right) E_{w}\left(\Delta^{\prime}\right)$, which is composed of sets with a diameter smaller than $c \cdot \delta$ as (3.46) shows. This establishes totally boundedness of the set

$$
E_{w}\left(\Delta^{\prime}\right) \pi_{w}\left(\mathfrak{A}_{r}(\mathcal{O})\right) E_{w}\left(\Delta^{\prime}\right) \subseteq \mathfrak{B}\left(\mathcal{H}_{w}\right) .
$$

By arbitrariness of $\Delta^{\prime}$ as well as of the bounded region $\mathcal{O}$, the representation $\left(\pi_{w}, \mathcal{H}_{w}\right)$ is thus seen to satisfy the Compactness Criterion of Fredenhagen and Hertel in the sense that the mappings

$$
T_{w, \Delta^{\prime}}^{\mathcal{O}}: \mathfrak{A}(\mathcal{O}) \rightarrow \mathfrak{B}\left(\mathcal{H}_{w}\right) \quad A \mapsto T_{w, \Delta^{\prime}}^{\mathcal{O}}(A) \doteq E_{w}\left(\Delta^{\prime}\right) \pi_{w}(A) E_{w}\left(\Delta^{\prime}\right)
$$

are altogether precompact.

(ii) According to the construction of $\left(\pi^{\bullet}, \mathcal{H}^{\bullet}, \mathfrak{A}^{\bullet}, \alpha^{\bullet}, \mathrm{P}^{c}, \overline{\mathfrak{L}_{0}^{c}}\right)$ from $\left(\pi_{w}, \mathcal{H}_{w}\right)$ explained in the proof of Theorem 4.3, both of these representations are related by the inequality

$$
\left\|E^{\bullet}\left(\Delta^{\prime}\right) \pi^{\bullet}(A) E^{\bullet}\left(\Delta^{\prime}\right)\right\| \leqslant\left\|E_{w}\left(\Delta^{\prime}\right) \pi_{w}(A) E_{w}\left(\Delta^{\prime}\right)\right\|,
$$

which holds for any $A \in \mathfrak{A}^{\bullet}$. Then $\Delta$-boundedness of the underlying particle weight again implies the existence of a bounded Borel set $\bar{\Delta} \supseteq \Delta+\Delta^{\prime}$ such that

$$
\left\|E^{\bullet}\left(\Delta^{\prime}\right) \pi^{\bullet}(A) E^{\bullet}\left(\Delta^{\prime}\right)\right\| \leqslant c \cdot\|E(\bar{\Delta}) A E(\bar{\Delta})\| .
$$

This relation replaces (3.46) in the proof of the first part, so that we conclude that indeed $\left(\pi^{\bullet}, \mathcal{H}^{\bullet}\right)$ inherits the precompactness properties of the underlying quantum field theory in the sense that all the sets

$$
E^{\bullet}\left(\Delta^{\prime}\right) \pi^{\bullet}\left(\mathfrak{A}_{r}^{\bullet}\left(\mathcal{O}_{k}\right)\right) E^{\bullet}\left(\Delta^{\prime}\right) \subseteq \mathfrak{B}\left(\mathcal{H}^{\bullet}\right)
$$

are totally bounded for any $r>0$ whenever $\Delta^{\prime}$ is an arbitrary bounded Borel set and $\mathcal{O}_{k}$ is one of the countably many localization regions from $\mathcal{R}^{c}$. Again that is sufficient to establish the fact that the Fredenhagen-Hertel Compactness Condition is satisfied in the restricted setting for Local Quantum Physics introduced in Section 4.1. 
Under the presupposition of the Compactness Criterion, a result corresponding to Proposition 5.1 can be proved for the irreducible representations $\left(\pi_{\xi}, \mathcal{H}_{\xi}\right)$ arising in the spatial disintegration of the restricted $\overline{\mathfrak{L}_{0}^{c}}$-particle weight $\left(\pi^{\bullet}, \mathcal{H}^{\bullet}, \mathfrak{A}^{\bullet}, \alpha^{\bullet}, \mathrm{P}^{c}, \overline{\mathfrak{L}_{0}^{c}}\right)$ by virtue of Theorem 4.4 if the domain of $\xi$ is further astricted to a $v$-measurable non-null subset $X_{0}$ of $X$.

Proposition 5.2. Let $\left(\pi_{w}, \mathcal{H}_{w}\right)$ be the GNS-representation of the quasi-local algebra $\mathfrak{A}$ corresponding to the $\Delta$-bounded particle weight $\langle. \mid$.$\rangle , and let \left(\pi^{\bullet}, \mathcal{H}^{\bullet}, \mathfrak{A}^{\bullet}, \alpha^{\bullet}, \mathrm{P}^{c}, \overline{\mathfrak{L}_{0}^{c}}\right)$ denote the restricted $\overline{\mathfrak{L}_{0}^{c}}$-particle weight associated with it according to Theorem 4.3. Under the hypothesis that the Compactness Criterion of Fredenhagen and Hertel is in force in the underlying quantum field theory, $v$-almost all of the irreducible representations $\left(\pi_{\xi}, \mathcal{H}_{\xi}\right)$ occurring in the spatial disintegration (4.13a) of $\left(\pi^{\bullet}, \mathcal{H}^{\bullet}\right)$ by course of Theorem 4.4 comply with this condition as well.

Proof. Select a dense sequence $\left\{A_{k}\right\}_{k \in \mathbb{N}}$ in the norm-separable $C^{*}$-algebra $\mathfrak{A}^{\bullet}$ and consider the countable set of compact balls $\Gamma_{N}$ of radius $N$ in $\mathbb{R}^{s+1}$. The corresponding operators $E^{\bullet}\left(\Gamma_{N}\right) \pi^{\bullet}\left(A_{k}\right) E^{\bullet}\left(\Gamma_{N}\right) \in \mathfrak{B}\left(\mathcal{H}^{\bullet}\right)$ are decomposable according to Theorem 4.4:

$$
W E^{\bullet}\left(\Gamma_{N}\right) \pi^{\bullet}\left(A_{k}\right) E^{\bullet}\left(\Gamma_{N}\right) W^{*}=\int_{\mathrm{X}}^{\oplus} d v(\xi) E_{\xi}\left(\Gamma_{N}\right) \pi_{\xi}\left(A_{k}\right) E_{\xi}\left(\Gamma_{N}\right)
$$

and [24, Section II.2.3, Proposition 2] tells us that the respective norms are related in compliance with the equation

$$
\left\|E^{\bullet}\left(\Gamma_{N}\right) \pi^{\bullet}\left(A_{k}\right) E^{\bullet}\left(\Gamma_{N}\right)\right\|=\text { v-ess } \sup \left\{\left\|E_{\xi}\left(\Gamma_{N}\right) \pi_{\xi}\left(A_{k}\right) E_{\xi}\left(\Gamma_{N}\right)\right\|: \xi \in \mathrm{X}\right\} .
$$

With regard to all possible combinations of operators $A_{k}$ and compact balls $\Gamma_{N}$ we thus infer that there exists a measurable non-null subset $X_{0}$ of $X$ such that for all $\xi \in X_{0}$ and all indices $k$ and $N$ the estimate

$$
\left\|E_{\xi}\left(\Gamma_{N}\right) \pi_{\xi}\left(A_{k}\right) E_{\xi}\left(\Gamma_{N}\right)\right\| \leqslant\left\|E^{\bullet}\left(\Gamma_{N}\right) \pi^{\bullet}\left(A_{k}\right) E^{\bullet}\left(\Gamma_{N}\right)\right\|
$$

holds. Now, let $\Delta^{\prime}$ be an arbitrary bounded Borel set which is thus contained in a compact ball $\Gamma_{N_{0}}$ and note that, by continuity of the representations $\pi_{\xi}$ and $\pi^{\bullet}$, the inequality (5.3) extends to arbitrary operators $A \in \mathfrak{A}^{\bullet}$. Therefore

$$
\left\|E_{\xi}\left(\Delta^{\prime}\right) \pi_{\xi}(A) E_{\xi}\left(\Delta^{\prime}\right)\right\| \leqslant\left\|E_{\xi}\left(\Gamma_{N_{0}}\right) \pi_{\xi}(A) E_{\xi}\left(\Gamma_{N_{0}}\right)\right\| \leqslant\left\|E^{\bullet}\left(\Gamma_{N_{0}}\right) \pi^{\bullet}(A) E^{\bullet}\left(\Gamma_{N_{0}}\right)\right\|,
$$

and this implies, according to (5.1b), the existence of a bounded Borel set $\bar{\Delta} \supseteq \Delta+\Delta^{\prime}$ such that

$$
\left\|E_{\xi}\left(\Delta^{\prime}\right) \pi_{\xi}(A) E_{\xi}\left(\Delta^{\prime}\right)\right\| \leqslant c \cdot\|E(\bar{\Delta}) A E(\bar{\Delta})\| .
$$

The arguments given in the proof of Proposition 5.1 can then again be applied to the present situation to show that for $\xi \in X_{0}$ the irreducible representations $\left(\pi_{\xi}, \mathcal{H}_{\xi}\right)$ altogether meet the requirements of the Fredenhagen-Hertel Compactness Condition. 
The central result of the present chapter is the perception that, under the above assumptions on the structure of phase space, the representations of the quasi-local $C^{*}$ algebras $\mathfrak{A}$ and $\mathfrak{A}^{\bullet}$ which we have come across, i. e. $\left(\pi_{w}, \mathcal{H}_{w}\right)$ and $\left(\pi^{\bullet}, \mathcal{H}^{\bullet}\right)$, respectively, as well as $v$-almost all of the irreducible representations $\left(\pi_{\xi}, \mathcal{H}_{\xi}\right)$ occurring in the direct integral decomposition of the latter, are locally normal. The representations of $\mathfrak{A}^{\bullet}$ can thus be continuously extended to all of $\mathfrak{A}$ in such a way that the formula (4.13a) describing the disintegration stays valid for the extended representations when $X$ is replaced by the non-null set $X_{0}$ occurring in Proposition 5.2.

Theorem 5.3 (Local Normality). Under the presumptions of Proposition 5.2 the following assertions are valid:

(i) The GNS-representation $\left(\pi_{w}, \mathcal{H}_{w}\right)$ of the quasi-local algebra $\mathfrak{A}$ is locally normal, i. e. continuous with respect to the relative $\sigma$-weak topologies of both $\mathfrak{A}(\mathcal{O}) \subseteq \mathfrak{B}(\mathcal{H})$ and $\pi_{w}(\mathfrak{A}(\mathcal{O})) \subseteq \mathfrak{B}\left(\mathcal{H}_{w}\right)$ for arbitrary bounded regions $\mathcal{O}$.

(ii) The representation $\left(\pi^{\bullet}, \mathcal{H}^{\bullet}\right)$ of the quasi-local algebra $\mathfrak{A}^{\bullet}$ is locally normal (continuous with respect to the relative $\sigma$-weak topologies of both $\mathfrak{A} \bullet\left(\mathcal{O}_{k}\right) \subseteq \mathfrak{B}(\mathcal{H})$ and $\pi^{\bullet}\left(\mathfrak{A}^{\bullet}\left(\mathcal{O}_{k}\right)\right) \subseteq \mathfrak{B}\left(\mathcal{H}^{\bullet}\right)$ for arbitrary bounded regions $\left.\mathcal{O}_{k} \in \mathcal{R}^{c}\right)$. The same holds true for the irreducible representations $\left(\pi_{\xi}, \mathcal{H}_{\xi}\right)$ occurring in the spatial disintegration of $\left(\pi^{\bullet}, \mathcal{H}^{\bullet}\right)$ when the indices $\xi$ are astricted to $\mathrm{X}_{0}$.

(iii) The representation $\left(\pi^{\bullet}, \mathcal{H}^{\bullet}\right)$ as well as the irreducible ones $\left(\pi_{\xi}, \mathcal{H}_{\xi}\right)$ with $\xi \in X_{0}$ allow for unique locally normal extensions to the whole of the original quasi-local algebra $\mathfrak{A}$ designated $\left(\bar{\pi}^{\bullet}, \mathcal{H}^{\bullet}\right)$ and $\left(\bar{\pi}_{\xi}, \mathcal{H}_{\xi}\right)$, respectively, which are related by

$$
\left(\bar{\pi}^{\bullet}, \mathcal{H}^{\bullet}\right) \simeq \int_{X_{0}}^{\oplus} d v(\xi)\left(\bar{\pi}_{\xi}, \mathcal{H}_{\xi}\right),
$$

where the representations $\left(\bar{\pi}_{\xi}, \mathcal{H}_{\xi}\right)$ are again irreducible.

Proof. (i) Let $\bar{\Delta}$ be a bounded Borel set and suppose that $\rho$ is a normal functional on $\mathfrak{B}(\mathcal{H})$. Then the same applies to the functional $\rho_{\bar{\Delta}}(.) \doteq \rho(E(\bar{\Delta}) . E(\bar{\Delta}))$, and therefore the mapping

$$
T_{\bar{\Delta}}: \mathfrak{A} \rightarrow \mathfrak{B}(\mathcal{H}) \quad A \mapsto T_{\bar{\Delta}}(A) \doteq E(\bar{\Delta}) A E(\bar{\Delta})
$$

is continuous with respect to the relative $\sigma$-weak topology of $\mathfrak{A}$. Now, according to the Compactness Condition, $T_{\bar{\Delta}}\left\lceil\mathfrak{A}(\mathcal{O})=T_{\bar{\Delta}}^{\mathcal{O}}\right.$ maps the unit ball $\mathfrak{A}_{1}(\mathcal{O})$ of the local $C^{*}$ algebra $\mathfrak{A}(\mathcal{O})$ onto the relatively compact set $E(\bar{\Delta}) \mathfrak{A}_{1}(\mathcal{O}) E(\bar{\Delta})$. The restriction of $T_{\bar{\Delta}}^{\mathcal{O}}$ to $\mathfrak{A}_{1}(\mathcal{O})$ is now obviously continuous with respect to the relative $\sigma$-weak topologies, but this result can be tightened up in the following sense: The relative $\sigma$-weak topology, being Hausdorff and coarser than the relative norm topology, and the relative uniform topology itself coincide on the compact norm closure of $E(\bar{\Delta}) \mathfrak{A}_{1}(\mathcal{O}) E(\bar{\Delta})$ due to a conclusion of general topology [44, Chapter One, $\S 3,2 .(6)]$. Therefore $T_{\bar{\Delta}}^{\mathcal{O}}$ is still continuous on $\mathfrak{A}_{1}(\mathcal{O})$ when its image is furnished with the norm topology instead.

Now, suppose that $\Delta^{\prime}$ is an arbitrary bounded Borel set and let $\bar{\Delta} \supseteq \Delta+\Delta^{\prime}$ be another bounded Borel set with the property that (3.46) is satisfied. Then the linear mapping

$$
E(\bar{\Delta}) A E(\bar{\Delta}) \mapsto E_{w}\left(\Delta^{\prime}\right) \pi_{w}(A) E_{w}\left(\Delta^{\prime}\right)
$$


is well-defined and continuous with respect to the uniform topologies of both domain and image. Therefore, as a consequence of the previous paragraph, we infer that the composition of this map with the restriction of $T_{\bar{\Delta}}$ to $\mathfrak{A}_{1}(\mathcal{O})$ is continuous, when $\mathfrak{A}_{1}(\mathcal{O})$ is endowed with the $\sigma$-weak topology whereas the range carries the relative norm topology. The resulting map is explicitly determined as the restriction to $\mathfrak{A}_{1}(\mathcal{O})$ of

$$
\pi_{w, \Delta^{\prime}}: \mathfrak{A} \rightarrow \mathfrak{B}\left(\mathcal{H}_{w}\right) \quad A \mapsto \pi_{w, \Delta^{\prime}}(A) \doteq E_{w}\left(\Delta^{\prime}\right) \pi_{w}(A) E_{w}\left(\Delta^{\prime}\right) .
$$

If $\eta$ denotes a $\sigma$-weakly continuous functional on $\mathfrak{B}\left(\mathcal{H}_{w}\right)$, the same is true regarding $\eta_{\Delta^{\prime}}(.) \doteq \eta\left(E_{w}\left(\Delta^{\prime}\right) . E_{w}\left(\Delta^{\prime}\right)\right)$ for any bounded Borel set $\Delta^{\prime} \subseteq \mathbb{R}^{s+1}$, and moreover, due to strong continuity of the spectral measure, $\eta$ is the uniform limit of the net of functionals $\eta_{\Delta^{\prime}}$ for $\Delta^{\prime} \nearrow \mathbb{R}^{s+1}$. Given a $\sigma$-weakly convergent net $\left\{A_{\imath}: \imath \in J\right\} \subseteq \mathfrak{A}_{1}(\mathcal{O})$ with limit $A \in \mathfrak{A}_{1}(\mathcal{O})$, we conclude from the discussion in the preceding paragraph that

$$
\eta_{\Delta^{\prime}}\left(\pi_{w}\left(A_{\imath}-A\right)\right)=\eta\left(\pi_{w, \Delta^{\prime}}\left(A_{\imath}-A\right)\right) \underset{\imath \in J}{\longrightarrow} 0
$$

Therefore, by means of the estimate

$$
\begin{aligned}
& \left|\eta \circ \pi_{w}\left(A_{\imath}-A\right)\right| \leqslant\left|\eta\left(\pi_{w}\left(A_{\imath}-A\right)\right)-\eta_{\Delta^{\prime}}\left(\pi_{w}\left(A_{\imath}-A\right)\right)\right|+\left|\eta_{\Delta^{\prime}}\left(\pi_{w}\left(A_{\imath}-A\right)\right)\right| \\
& \quad \leqslant \| \eta-\eta_{\Delta^{\prime}}|||| \pi_{w}\left(A_{\imath}-A\right)||+\left|\eta_{\Delta^{\prime}}\left(\pi_{w}\left(A_{\imath}-A\right)\right)\right| \leqslant 2|| \eta-\eta_{\Delta^{\prime}}||+\left|\eta_{\Delta^{\prime}}\left(\pi_{w}\left(A_{\imath}-A\right)\right)\right|,
\end{aligned}
$$

it is easily seen that, upon selection of a suitable bounded Borel set $\Delta^{\prime}$, the right-hand side can be made smaller than any given bound for $\imath \succ \mathfrak{l}_{0}$ with an appropriate index $\mathfrak{l}_{0}$. This being true for any $\sigma$-weakly continuous functional $\eta$ on $\mathfrak{B}\left(\mathcal{H}_{w}\right)$ and arbitrary nets $\left\{A_{\imath}: \imath \in J\right\}$ in $\mathfrak{A}_{1}(\mathcal{O})$ converging to $A \in \mathfrak{A}_{1}(\mathcal{O})$ with respect to the $\sigma$-weak topology of $\mathfrak{B}(\mathcal{H})$, we have thus established that the restrictions of the representation $\pi_{w}$ to each of the unit balls $\mathfrak{A}_{1}(\mathcal{O})$ are $\sigma$-weakly continuous. According to [43, Lemma 10.1.10] this assertion extends to the entire local $C^{*}$-algebra $\mathfrak{A}(\mathcal{O})$, so that $\pi_{w}$ indeed turns out to be locally normal.

(ii) The arguments given above in the case of $\pi_{w}$ can be transferred literally to the representations $\pi^{\bullet}$ and $\pi_{\xi}, \xi \in \mathrm{X}_{0}$, in view of the relations (5.1b) and (5.4b) established in the proofs of Propositions 5.1 and 5.2, which substitute (3.46) used in the first part. The evident modifications to be applied include the restriction to local algebras $\mathfrak{A}^{\bullet}\left(\mathcal{O}_{k}\right)$ where $\mathcal{O}_{k}$ is a member of the countable family $\mathcal{R}^{c}$.

(iii) Complementary to the statements of the second part, [43, Lemma 10.1.10] exhibits that the representations $\pi^{\bullet}$ and $\pi_{\xi}, \xi \in X_{0}$, allow for unique $\sigma$-weakly continuous extensions $\bar{\pi}^{\bullet}$ and $\bar{\pi}_{\xi}$ onto the weak closures $\mathfrak{A} \bullet\left(\mathcal{O}_{k}\right)^{\prime \prime}$ [11, Corollary 2.4.15] of the local algebras, which, due to the Bicommutant Theorem [11, Theorem 2.4.11], coincide with the strong closures and thus, by the very construction of $\mathfrak{A} \bullet\left(\mathcal{O}_{k}\right), \mathcal{O}_{k} \in \mathcal{R}^{c}$, expounded in Section 4.1, contain the corresponding local $C^{*}$-algebras $\mathfrak{A}\left(\mathcal{O}_{k}\right)$ of the underlying quantum field theory. Now, due to the net structure of $\mathcal{O}_{k} \mapsto \mathfrak{A}\left(\mathcal{O}_{k}\right)$, the quasi-local $C^{*}$ algebra $\mathfrak{A}$ is its $C^{*}$-inductive limit, i. e. the norm closure of the *-algebra $\bigcup_{\mathcal{O}_{k} \in \mathfrak{R}^{c}} \mathfrak{A}\left(\mathcal{O}_{k}\right)$. As the representations $\bar{\pi}^{\bullet}$ and $\bar{\pi}_{\xi}, \xi \in X_{0}$, are altogether uniformly continuous on this *-algebra [48, Theorem 1.5.7], they can in a unique way be continuously extended to its completion $\mathfrak{A}$ [44, Chapter One, $\S 5,4 .(4)]$, and these extensions, again denoted $\bar{\pi}^{\bullet}$ and $\bar{\pi}_{\xi}$, respectively, are easily seen to be compatible with the algebraic structure of 
A. $\left(\bar{\pi}^{\bullet}, \mathcal{H}^{\bullet}\right)$ and $\left(\bar{\pi}_{\xi}, \mathcal{H}_{\xi}\right)$ are thus representations of this quasi-local algebra, evidently irreducible in the case of $\bar{\pi}_{\xi}$, and moreover locally normal, since, by construction, they are $\sigma$-weakly continuous when restricted to local algebras $\mathfrak{A}\left(\mathcal{O}_{k}\right)$ pertaining to the countable subclass of regions $\mathcal{O}_{k} \in \mathcal{R}^{c}$, and an arbitrary local algebra $\mathfrak{A}(\mathcal{O})$ is contained in at least one of these. The statement on uniqueness of the extensions is then an immediate consequence of the fact that they are uniquely determined by the property of being $\sigma$-weakly continuous on the local $C^{*}$-algebras $\mathfrak{A}\left(\mathcal{O}_{k}\right)$.

Regarding the disintegration of operators $\bar{\pi}^{\bullet}(A)$ for arbitrary $A \in \mathfrak{A}$, note that any operator $B \in \mathfrak{A}\left(\mathcal{O}_{k}\right)$ is the $\sigma$-weak limit of a sequence $\left\{B_{n}\right\}_{n \in \mathbb{N}}$ in $\mathfrak{A}_{r}^{\bullet}\left(\mathcal{O}_{k}\right)$ with $r=\|B\|$. For nets in $\mathfrak{A}_{r}^{\bullet}\left(\mathcal{O}_{k}\right)$ this statement is a consequence of Kaplansky's Density Theorem [54, Theorem II.4.8] in connection with [54, Lemma II.2.5] and the various relations between the different locally convex topologies on $\mathfrak{B}(\mathcal{H})$. The specialization to sequences is justified by [54, Proposition II.2.7] in view of the separability of $\mathcal{H}$. The operators $L \in \mathfrak{L}^{c}$ define fundamental sequences of measurable vector fields $\left\{|L\rangle_{\xi}: \xi \in \mathrm{X}_{0}\right\}$ (cf. [24, Section II.1.3, Definition 1]) and, as the operators $\pi^{\bullet}\left(B_{n}\right)$ are decomposable, all the functions

$$
h_{n}: X_{0} \rightarrow \mathbb{C} \quad \xi \mapsto h_{n}(\xi) \doteq\left\langle L_{1}\left|\pi_{\xi}\left(B_{n}\right)\right| L_{2}\right\rangle_{\xi}
$$

are measurable for arbitrary $L_{1}, L_{2} \in \mathfrak{L}^{c}$. The same is valid for the pointwise limit of this sequence [26, II.1.10]

$$
h: X_{0} \rightarrow \mathbb{C} \quad \xi \mapsto h(\xi) \doteq\left\langle L_{1}\left|\bar{\pi}_{\xi}(B)\right| L_{2}\right\rangle_{\xi},
$$

and that suffices, according to [24, Section II.2.1, Proposition 1], to demonstrate that $\left\{\bar{\pi}_{\xi}(B): \xi \in X_{0}\right\}$ is a measurable field of operators. As the sequence $\left\{\pi^{\bullet}\left(B_{n}\right)\right\}_{n \in \mathbb{N}}$ converges $\sigma$-weakly to $\bar{\pi}^{\bullet}(B)$ by assumption and since, moreover, $v\left(X_{0}\right)$ is finite and the family of operators $\left\{\pi_{\xi}\left(B_{n}\right): \xi \in X_{0}\right\}$ is bounded by $\|B\|$ for any $n$, we conclude with Lebesgue's Dominated Convergence Theorem applied to the decompositions of $\pi^{\bullet}\left(B_{n}\right)$ with respect to $\mathrm{X}_{0}$ (which differs from $\mathrm{X}$ only by a null set), that

$$
\begin{aligned}
\left\langle L_{1}\left|\pi^{\bullet}\left(B_{n}\right)\right| L_{2}\right\rangle=\int_{X_{0}} d v(\xi)\left\langle L_{1}\left|\pi_{\xi}\left(B_{n}\right)\right| L_{2}\right\rangle_{\xi} \\
\underset{n \rightarrow \infty}{\longrightarrow} \int_{X_{0}} d v(\xi)\left\langle L_{1}\left|\bar{\pi}_{\xi}(B)\right| L_{2}\right\rangle_{\xi}=\left\langle L_{1}\left|\bar{\pi}^{\bullet}(B)\right| L_{2}\right\rangle .
\end{aligned}
$$

If $W_{0}$ denotes the isometry which implements the unitary equivalence

$$
\left(\pi^{\bullet}, \mathcal{H}^{\bullet}\right) \simeq \int_{\mathrm{X}_{0}}^{\oplus} d v(\xi)\left(\pi_{\xi}, \mathcal{H}_{\xi}\right)
$$

and has all the properties of the operator $W$ introduced in Theorem 4.4, then, by density of the set $\left\{|L\rangle^{\bullet}: L \in \mathfrak{L}^{c}\right\}$ in $\mathcal{H}^{\bullet}$, we infer from (5.10) that

$$
W_{0} \bar{\pi}^{\bullet}(B) W_{0}^{*}=\int_{X_{0}}^{\oplus} d v(\xi) \bar{\pi}_{\xi}(B)
$$

This relation has been established under the presupposition that $B$ belongs to some local $C^{*}$-algebra $\mathfrak{A}\left(\mathcal{O}_{k}\right)$. Now, it is possible to reapply the above reasoning in the case of an 
arbitrary element $A$ of the quasi-local algebra $\mathfrak{A}$, which can be approximated uniformly by a sequence $\left\{A_{n}\right\}_{n \in \mathbb{N}}$ from $\bigcup_{\mathcal{O}_{k} \in \mathcal{R}^{c}} \mathfrak{A}\left(\mathcal{O}_{k}\right)$. In this way, (5.11a) is extended to all of $\mathfrak{A}$ so that we end up with the final equation

$$
W_{0} \bar{\pi}^{\bullet}(A) W_{0}^{*}=\int_{\mathrm{X}_{0}}^{\oplus} d v(\xi) \bar{\pi}_{\xi}(A), \quad A \in \mathfrak{A},
$$

demonstrating that indeed

$$
\left(\bar{\pi}^{\bullet}, \mathcal{H}^{\bullet}\right) \simeq \int_{\mathrm{X}_{0}}^{\oplus} d v(\xi)\left(\bar{\pi}_{\xi}, \mathcal{H}_{\xi}\right)
$$

Theorem 5.3 shows that, under the assumption of sensible phase space restrictions, no information on a physical system described by a normal state of bounded energy $\omega \in \mathcal{S}(\Delta)$ gets lost in the entirety of constructions presented in Chapters 3 and 4 . These have led us from $\omega$ via an associated particle weight with representation $\left(\pi_{w}, \mathcal{H}_{w}\right)$ of the quasi-local algebra $\mathfrak{A}$ to the induced restricted $\overline{\mathfrak{L}_{0}^{c}}$-particle weight $\left(\pi^{\bullet}, \mathcal{H}^{\bullet}, \mathfrak{A}^{\bullet}, \alpha^{\bullet}, \mathrm{P}^{c}, \overline{\mathfrak{L}_{0}^{c}}\right)$, which comprises a representation $\left(\pi^{\bullet}, \mathcal{H}^{\bullet}\right)$ of the algebra $\mathfrak{A}^{\bullet}$ allowing for a disintegration in terms of irreducible representations $\left\{\left(\pi_{\xi}, \mathcal{H}_{\xi}\right): \xi \in X_{0}\right\}$. Then, according to the preceding theorem, this disintegration is extendable in a unique fashion to one in terms of locally normal representations of the original algebra $\mathfrak{A}$ as expressed by (5.5). Now, due to the explicit construction in Theorem 4.3 of $\left(\pi^{\bullet}, \mathcal{H}^{\bullet}\right)$ from $\left(\pi_{w}, \mathcal{H}_{w}\right)$, the local normality of both of these representations implies that, actually, $\bar{\pi}^{\bullet}$ coincides with the restriction of $\pi_{w}$ to the subspace $\mathcal{H}^{\bullet}$ of $\mathcal{H}_{w}$. Thus we arrive at a partial reconstruction of the GNS-representation $\left(\pi_{w}, \mathcal{H}_{w}\right)$, which only depends on the initial choice of a subspace of the Hilbert space $\mathcal{H}_{w}$. Moreover, by Theorem 5.3, this entails a spatial disintegration of $\Delta$-bounded particle weights $\langle. \mid$.$\rangle according to$ the following reformulation of (5.5):

$$
\left(\pi_{w}, \mathcal{H}^{\bullet}\right) \simeq \int_{\mathrm{X}_{0}}^{\oplus} d v(\xi)\left(\bar{\pi}_{\xi}, \mathcal{H}_{\xi}\right)
$$




\section{Chapter 6}

\section{Disintegration Revisited: Choquet Theory}

The spatial disintegration as expounded in Chapter 4 suffered from a couple of awkward drawbacks, which, in our belief, are inessential concomitants of this special approach to a decomposition theory for particle weights and have no bearing on the physical significance of the concept proper. It should be noted in this connection that, to be able to apply the standard disintegration theory for representations made available in the literature on $C^{*}$-algebras, we had to fall back upon separable constructs and countable dense subsets thereof. As a consequence it had to be accepted, that a theory of disintegration could only be formulated in terms of restricted $\overline{\mathfrak{L}_{0}^{c}}$-particle weights. But these technical difficulties seem to be accidental, and the question obtrudes on us if it is possible to carry through a disintegration, in the course of which no need arises to leave the class of particle weights proper, which means that the disintegration can indeed be formulated in terms of pure particle weights. This is the topic of the present chapter, presenting the partial results we were able to produce in this direction to date.

As already noted in the remark following Definition 3.14, the totality of particle weights constitutes a positive proper convex cone when supplemented by the trivial form. This observation opens the way to an application of another concept of disintegration: the barycentric decomposition in the special form of a generalization of the well-known Theorem of Krein-Milman [42, Theorem 1.4.3]. This approach, initiated by Choquet and further developed by Bishop and de Leeuw [8, 21], is especially welladapted to the study of convex sets in infinite-dimensional spaces. An introduction to this theory can be found in [1,50] and also in [11, Section 4.1.2] where it is applied to get a decomposition of states on the quasi-local $C^{*}$-algebra $\mathfrak{A}$ in terms of pure ones. The mathematical structure in this case is easily accessible from the point of view of Choquet theory:

- The positive linear functionals on $\mathfrak{A}$ constitute a positive convex cone $\mathrm{K}$ in its topological dual $\mathfrak{A}^{*}$.

- The quasi-local algebra contains a unit $\mathbf{1}$, which defines a base of this cone when it is considered as a continuous linear functional on $\mathfrak{A}^{*}$, thus introducing a convex function which intersects $K$. 
- This convex base of $\mathrm{K}$ coincides with the set of states on $\mathfrak{A}$ and thus turns out to be compact with respect to the weak* topology [11, Propositon 2.3.11 and Theorem 2.3.15].

The situation is much more complicated when particle weights are considered. These do constitute a positive cone $W$ in the space of sesquilinear forms on the left ideal $\mathfrak{L}$ of localizing operators; but one of the key features in the construction of an algebra of detectors was the absence of a unit element, the existence of which would produce infinite values of the asymptotic functionals $\sigma$ arising in Chapter 3. The obvious questions to be answered are:

- What is an appropriate (metrizable) topology $\mathcal{T}$ to be introduced on $W$ to render relevant subsets compact?

- How can a convex base $B_{W}$ be fitted into the cone $W$ in a physically meaningful way and such that this base is compact with respect to the beforementioned topology?

In our approach the class with respect to which the disintegration is to be performed will be further restricted (cf. Definition 6.6 below).

The answer to the first of the above problems which we are going to present in this chapter is based on an effective control of the dislocalization of almost local operators combined with the Fredenhagen-Hertel compactness condition. To be more specific, a norm will be introduced on the space $\mathfrak{L}_{0}$ of almost local vacuum annihilation operators which in a way measures their deviation from being contained in a local algebra. Making use of this norm on the set $\mathfrak{L}_{0}(\Gamma)$ of those operators with energy-momentum transfer in a compact and convex subset $\Gamma$ of $\complement \bar{V}_{+}$, it can be shown that $\mathfrak{L}_{0}(\Gamma)$ is small in the $q_{\Delta}$-topology under the assumption of the Compactness Criterion of Fredenhagen and Hertel. By its very definition, the notion of almost locality as introduced in Definition 2.1 imposes a condition of rapid decrease on the norm difference between almost local operators and strictly local ones according to the growing extension of the localization regions. This contrives to introduce the following norms on $\mathfrak{L}_{0}$.

Definition 6.1. Let $m$ be an arbitrary natural number, then the equation

$$
Q^{m}\left(L_{0}\right) \doteq \sup _{r>0} \inf \left\{r^{m}\left\|L_{0}-L_{r}\right\|: L_{r} \in \mathfrak{A}\left(\mathcal{O}_{r}\right)\right\}, \quad L_{0} \in \mathfrak{L}_{0},
$$

defines a norm on the vector space $\mathfrak{L}_{0}$.

Remark. The seminorm properties of the mapping $Q^{m}$ are self-evident, they even hold for arbitrary almost local operators as arguments. To infer $L_{0}=0$ from the condition $Q^{m}\left(L_{0}\right)=0$ one has to restrict attention to vacuum annihilation operators. The latter equation means that the operator $L_{0}$ is contained in the norm closure of any local algebra $\mathfrak{A}\left(\mathcal{O}_{r}\right), r>0$. As these are $C^{*}$-algebras, hence uniformly closed, $L_{0}$ itself turns out to be a local operator, a property which can be reconciled with it being a vacuum annihilation operator only for $L_{0}=0$.

The information concerning the localization of the operator $L_{0} \in \mathfrak{L}_{0}$ embodied by the value of $Q^{m}\left(L_{0}\right)$ is highly dependent on the norm which $L_{0}$ carries as an element of the $C^{*}$-algebra $\mathfrak{A}$. Therefore we combine both topologies in the subsequent definition. 
Definition 6.2. For any natural number $m$ a norm on $\mathfrak{L}_{0}$ is defined by

$$
\left\|L_{0}\right\|_{m} \doteq\left\|L_{0}\right\|+Q^{m}\left(L_{0}\right), \quad L_{0} \in \mathfrak{L}_{0} .
$$

As announced above this topology is now to be related to the $q_{\Delta}$-seminorms on the subspace $\mathfrak{L}_{0}(\Gamma)$, where $\Gamma$ denotes a compact, convex subset of the complement of the forward light cone. Although we have the inequality (2.10) at our disposition, we want to reformulate it here in order to make explicit the dependence of the integrand on its right-hand side upon the bounded Borel set $\Delta$ and upon the energy-momentum transfer $\Gamma$. To this end one has to reapply the arguments given in the Appendix of [15].

Proposition 6.3. Let $\Delta$ be a bounded Borel set and $\Gamma$ a compact and convex subset of $\complement \bar{V}_{+}$. There exists a bounded Borel set $\Delta^{\prime}(\Delta, \Gamma) \subseteq \mathbb{R}^{s+1}$, depending on $\Delta$ and $\Gamma$ only, such that for any $L_{0} \in \mathfrak{L}_{0}(\Gamma)$ there holds the estimate

$$
q_{\Delta}\left(L_{0}\right)^{2} \leqslant N^{\prime}(\Delta, \Gamma) \int_{\mathbb{R}^{s}} d^{s} x\left\|E\left(\Delta^{\prime}(\Delta, \Gamma)\right)\left[\alpha_{x}\left(L_{0}\right), L_{0}{ }^{*}\right] E\left(\Delta^{\prime}(\Delta, \Gamma)\right)\right\|
$$

with a suitable constant $N^{\prime}(\Delta, \Gamma)$, which is again specified by the sets $\Delta$ and $\Gamma$.

Proof. In a first step it will be shown that, setting

$$
Q_{\boldsymbol{K}} \doteq \int_{\boldsymbol{K}} d^{s} x \alpha_{\boldsymbol{x}}\left(L_{0}{ }^{*} L_{0}\right)
$$

for any compact subset $\boldsymbol{K}$ of $\mathbb{R}^{s}$, the following estimate is in force for arbitrary bounded Borel sets $\Delta_{0}$ :

$$
\left\|E\left(\Delta_{0}\right) Q_{\boldsymbol{K}} E\left(\Delta_{0}\right)\right\| \leqslant N^{\prime \prime} \int_{\boldsymbol{K}-\boldsymbol{K}} d^{s} x\left\|E\left(\Delta^{\prime \prime}\right)\left[\alpha_{x}\left(L_{0}\right), L_{0}{ }^{*}\right] E\left(\Delta^{\prime \prime}\right)\right\|
$$

with a suitable constant $N^{\prime \prime}$ and an appropriate bounded Borel set $\Delta^{\prime \prime}$. If $\omega_{\Psi}$ denotes a state on $\mathfrak{B}(\mathcal{H})$ which is induced by a vector $\Psi \in E\left(\Delta_{0}\right) \mathcal{H}$ we can immediately adopt the inequalities of $[15$, p. 640] to get

$$
\begin{aligned}
& \omega_{\Psi}\left(Q_{\boldsymbol{K}}\right)^{2} \leqslant \omega_{\Psi}\left(Q_{\boldsymbol{K}} \cdot Q_{\boldsymbol{K}}\right) \\
& \begin{aligned}
\leqslant \omega_{\Psi}\left(Q_{\boldsymbol{K}}\right) \cdot \sup _{\boldsymbol{y} \in \mathbb{R}^{s}} \int_{\boldsymbol{K}-\boldsymbol{K}} d^{s} x\left\|L_{0} U(-\boldsymbol{y}) \Psi\right\|^{-1}\left\|\left[\alpha_{\boldsymbol{x}}\left(L_{0}\right), L_{0}{ }^{*}\right] L_{0} U(-\boldsymbol{y}) \Psi\right\| \\
\\
\quad+\int_{\boldsymbol{K}} d^{s} x \omega_{\Psi}\left(\alpha_{x}\left(L_{0}{ }^{*}\right) Q_{\boldsymbol{K}} \alpha_{x}\left(L_{0}\right)\right) .
\end{aligned}
\end{aligned}
$$

The integrand of the second term on the right-hand side is subject to the relation

$$
\omega_{\Psi}\left(\alpha_{x}\left(L_{0}{ }^{*}\right) Q_{K} \alpha_{x}\left(L_{0}\right)\right) \leqslant\left\|E\left(\bar{\Delta}_{0}+\Gamma\right) Q_{K} E\left(\bar{\Delta}_{0}+\Gamma\right)\right\| \cdot \omega_{\Psi}\left(\alpha_{x}\left(L_{0}{ }^{*} L_{0}\right)\right)
$$

with $\bar{\Delta}_{0}$ denoting the closure of $\Delta_{0}$. Upon insertion into (6.5a), removal of the resulting common factor $\omega_{\Psi}\left(Q_{K}\right)$ on both sides and passing to the supremum with respect to all unit vectors $\Psi \in E\left(\Delta_{0}\right) \mathcal{H}$, we get

$$
\left\|E\left(\Delta_{0}\right) Q_{\boldsymbol{K}} E\left(\Delta_{0}\right)\right\| \leqslant \int_{\boldsymbol{K}-\boldsymbol{K}} d^{s} x\left\|\left[\alpha_{x}\left(L_{0}\right), L_{0}{ }^{*}\right] E\left(\bar{\Delta}_{0}+\Gamma\right)\right\|+\left\|E\left(\bar{\Delta}_{0}+\Gamma\right) Q_{\boldsymbol{K}} E\left(\bar{\Delta}_{0}+\Gamma\right)\right\|,
$$


where use is made of the fact that all the vectors $L_{0} U(-\boldsymbol{y}) \Psi$ belong to the subspace $E\left(\bar{\Delta}_{0}+\Gamma\right) \mathcal{H}$ for arbitrary $\boldsymbol{y} \in \mathbb{R}^{s}$ and $\Psi \in E\left(\Delta_{0}\right) \mathcal{H}$. The preparatory estimate (6.4) is now established by complete induction on $n$, where this natural number is defined in dependence on the sets $\Delta_{0}$ and $\Gamma$ through the condition $\left(\bar{\Delta}_{0}+\Gamma_{n}\right) \cap \bar{V}_{+}=\emptyset$ (cf. the proof of Proposition 2.6 on page 9).

For $n=1$ we have, according to the spectrum condition, $E\left(\bar{\Delta}_{0}+\Gamma\right)=0$ so that (6.4) is trivially fulfilled since its left-hand side vanishes. Now assume that the condition $\left(\bar{\Delta}_{0}+\Gamma_{n+1}\right) \cap \bar{V}_{+}=\emptyset$ is valid, which, stated another way, means that the intersection of $\left(\bar{\Delta}_{0}+\Gamma\right)+\Gamma_{n}$ with the complement of $\bar{V}_{+}$is empty. As $\bar{\Delta}_{0}+\Gamma$ is a bounded Borel set we can apply the induction hypothesis for $n$, i. e. (6.4) with $\Delta_{0}$ replaced by $\bar{\Delta}_{0}+\Gamma$, to infer that there exists a bounded Borel set $\Delta_{0}^{\prime \prime}$ which satisfies

$$
\left\|E\left(\bar{\Delta}_{0}+\Gamma\right) Q_{\boldsymbol{K}} E\left(\bar{\Delta}_{0}+\Gamma\right)\right\| \leqslant N_{0}^{\prime \prime} \int_{\boldsymbol{K}-\boldsymbol{K}} d^{s} x\left\|E\left(\Delta_{0}^{\prime \prime}\right)\left[\alpha_{x}\left(L_{0}\right), L_{0}^{*}\right] E\left(\Delta_{0}^{\prime \prime}\right)\right\|
$$

for an appropriate constant $N_{0}^{\prime \prime}$. This estimate inserted into (6.5c) amounts to

$$
\begin{aligned}
& \left\|E\left(\Delta_{0}\right) Q_{\boldsymbol{K}} E\left(\Delta_{0}\right)\right\| \\
& \quad \leqslant \int_{\boldsymbol{K}-\boldsymbol{K}} d^{s} x\left\|\left[\alpha_{x}\left(L_{0}\right), L_{0}^{*}\right] E\left(\bar{\Delta}_{0}+\Gamma\right)\right\|+N_{0}^{\prime \prime} \int_{\boldsymbol{K}-\boldsymbol{K}} d^{s} x\left\|E\left(\Delta_{0}^{\prime \prime}\right)\left[\alpha_{x}\left(L_{0}\right), L_{0}^{*}\right] E\left(\Delta_{0}^{\prime \prime}\right)\right\|,
\end{aligned}
$$

from which to conclude the validity of (6.4) with suitable constant $N^{\prime \prime}=N_{0}^{\prime \prime}+1$ and proper bounded Borel set $\Delta^{\prime \prime}=\Delta_{0}^{\prime \prime} \cup\left(\left(\bar{\Delta}_{0}+\Gamma\right)+\Gamma-\Gamma\right)$ is an obvious task.

Now, having established (6.4), we can specialize it to $\Delta_{0} \doteq \Delta$ and pass to the limit $\boldsymbol{K} \nearrow \mathbb{R}^{s}$ as in the proof of Proposition 2.6, noting that

$$
Q_{\Delta, \boldsymbol{K}}^{\left(L_{0}^{*} L_{0}\right)}=E(\Delta) Q_{\boldsymbol{K}} E(\Delta)
$$

and that, due to almost locality of $L_{0}$, the integral on the right-hand side can be extended over all of $\mathbb{R}^{s}$. As a result, in view of Definition 2.8, one arrives at the desired inequality (6.3), where the formulation chosen makes explicit its dependence on $\Delta$ and $\Gamma$.

The formula (6.3) just established is to be applied in the sequel to produce an estimate of the seminorm $q_{\Delta}\left(L_{0}\right)$ for operators $L_{0} \in \mathfrak{L}_{0}(\Gamma)$ with compact and convex $\Gamma \subseteq \complement \bar{V}_{+}$in terms of the initial operator norm $\|$.$\| and of the norm Q^{m}($.$) introduced$ in Definition 6.1. In order to get a manageable result we specialize to the case $m=2 s$.

Lemma 6.4. Let $\Delta$ be a bounded Borel set and $\Gamma$ a compact and convex subset of the complement of $\bar{V}_{+}$. Then there exists a bounded Borel set $\Delta^{\prime \prime}(\Delta, \Gamma)$, depending on $\Delta$ and $\Gamma$, such that for any $L_{0} \in \mathfrak{L}_{0}(\Gamma)$ the estimate

$$
\begin{aligned}
q_{\Delta}\left(L_{0}\right) \leqslant N^{\prime}(\Delta, \Gamma)^{1 / 2}\left(a(s)\left\|L_{0}\right\|^{2}+\right. & \left.b(s)\left\|L_{0}\right\|+c(s)\right)^{1 / 2} . \\
& Q^{2 s}\left(L_{0}\right)^{1 / 4}\left\|E\left(\Delta^{\prime \prime}(\Delta, \Gamma)\right) L_{0} E\left(\Delta^{\prime \prime}(\Delta, \Gamma)\right)\right\|^{1 / 2}
\end{aligned}
$$

holds with suitable coefficients depending on the spatial dimension s. 
Proof. We have to calculate the integral on the right-hand side of (6.3) and, to do so, it is split into two parts according to $|\boldsymbol{x}|>R$ or $|\boldsymbol{x}| \leqslant R$ with an abitrary radius $R$ which is held fixed for the moment. For large $|\boldsymbol{x}|$ we use the estimate (2.2a) for the integrand and get in terms of the norm $Q^{2 s}$ :

$$
\begin{aligned}
& \left\|E\left(\Delta^{\prime}(\Delta, \Gamma)\right)\left[\alpha_{x}\left(L_{0}\right), L_{0}{ }^{*}\right] E\left(\Delta^{\prime}(\Delta, \Gamma)\right)\right\| \\
& \leqslant\left\|\left[\alpha_{x}\left(L_{0}\right), L_{0}^{*}\right]\right\| \leqslant 4\left\|L_{0}\right\|\left\|L_{0}-\left(L_{0}\right)_{2^{-1}|x|}\right\|+2\left\|L_{0}-\left(L_{0}\right)_{2^{-1}|x|}\right\|^{2} \\
& \leqslant 4\left\|L_{0}\right\| 2^{2 s}|\boldsymbol{x}|^{-2 s} Q^{2 s}\left(L_{0}\right)+22^{4 s}|\boldsymbol{x}|^{-4 s} Q^{2 s}\left(L_{0}\right)^{2} \text {. }
\end{aligned}
$$

Accordingly, the respective integral is subject to the inequality

$$
\begin{aligned}
& \int_{|x|>R} d^{s} x\left\|E\left(\Delta^{\prime}(\Delta, \Gamma)\right)\left[\alpha_{x}\left(L_{0}\right), L_{0}^{*}\right] E\left(\Delta^{\prime}(\Delta, \Gamma)\right)\right\| \\
& \quad \leqslant 2^{2 s+2}\left\|L_{0}\right\| Q^{2 s}\left(L_{0}\right) \int_{|x|>R} d^{s} x|x|^{-2 s}+2^{4 s+1} Q^{2 s}\left(L_{0}\right)^{2} \int_{|x|>R} d^{s} x|x|^{-4 s} .
\end{aligned}
$$

The integrand for small $|\boldsymbol{x}|$ is evaluated observing the spectral projections arising on the right-hand side of (6.3), which are abbreviated as $\Delta^{\prime} \doteq \Delta^{\prime}(\Delta, \Gamma)$ with closure $\overline{\Delta^{\prime}}$. This leads to

$$
\begin{aligned}
& \left\|E\left(\Delta^{\prime}(\Delta, \Gamma)\right)\left[\alpha_{x}\left(L_{0}\right), L_{0}{ }^{*}\right] E\left(\Delta^{\prime}(\Delta, \Gamma)\right)\right\| \\
& \leqslant\left\|E\left(\Delta^{\prime}\right) \alpha_{x}\left(L_{0}\right) E\left(\overline{\Delta^{\prime}}-\Gamma\right) L_{0}{ }^{*} E\left(\Delta^{\prime}\right)\right\|+\left\|E\left(\Delta^{\prime}\right) L_{0}{ }^{*} E\left(\overline{\Delta^{\prime}}+\Gamma\right) \alpha_{x}\left(L_{0}\right) E\left(\Delta^{\prime}\right)\right\| \\
& \leqslant\left\|E\left(\Delta^{\prime} \cup\left(\overline{\Delta^{\prime}}-\Gamma\right)\right) L_{0} E\left(\Delta^{\prime} \cup\left(\overline{\Delta^{\prime}}-\Gamma\right)\right)\right\|^{2}+\left\|E\left(\Delta^{\prime} \cup\left(\overline{\Delta^{\prime}}+\Gamma\right)\right) L_{0} E\left(\Delta^{\prime} \cup\left(\overline{\Delta^{\prime}}+\Gamma\right)\right)\right\|^{2} \\
& \quad \leqslant 2\left\|E\left(\Delta^{\prime \prime}(\Delta, \Gamma)\right) L_{0} E\left(\Delta^{\prime \prime}(\Delta, \Gamma)\right)\right\|^{2} \quad(6.9 a)
\end{aligned}
$$

where $\Delta^{\prime \prime}(\Delta, \Gamma) \doteq \Delta \cup(\bar{\Delta}+\Gamma) \cup\left(\Delta^{\prime} \cup\left(\overline{\Delta^{\prime}}-\Gamma\right)\right) \cup\left(\Delta^{\prime} \cup\left(\overline{\Delta^{\prime}}+\Gamma\right)\right)$ - the inclusion of $\Delta \cup$ $(\bar{\Delta}+\Gamma)$ into this definition being required at the very end of the present argumentation. The corresponding integral satisfies the inequality

$$
\begin{aligned}
\int_{|x| \leqslant R} d^{s} x\left\|E\left(\Delta^{\prime}(\Delta, \Gamma)\right)\left[\alpha_{x}\left(L_{0}\right), L_{0}{ }^{*}\right] E\left(\Delta^{\prime}(\Delta, \Gamma)\right)\right\| & \\
& \leqslant 2\left\|E\left(\Delta^{\prime \prime}(\Delta, \Gamma)\right) L_{0} E\left(\Delta^{\prime \prime}(\Delta, \Gamma)\right)\right\|^{2} \int_{|x| \leqslant R} d^{s} x .
\end{aligned}
$$

The integrals remaining in (6.8b) and (6.9b) are known from calculus (cf. [22, Section 4.11]):

$$
\begin{aligned}
\int_{|x|>R} d^{s} x|\boldsymbol{x}|^{-2 s} & =\omega_{s} \int_{R}^{\infty} d r r^{s-1} r^{-2 s}=s^{-1} \omega_{s} R^{-s}, \\
\int_{|x|>R} d^{s} x|\boldsymbol{x}|^{-4 s} & =\omega_{s} \int_{R}^{\infty} d r r^{s-1} r^{-4 s}=(3 s)^{-1} \omega_{s} R^{-3 s}, \\
\int_{|x| \leqslant R} d^{s} x & =\omega_{s} \int_{0}^{R} d r r^{s-1}=s^{-1} \omega_{s} R^{s},
\end{aligned}
$$

where the factor $\omega_{s}$ is defined via the $\Gamma$-function as

$$
\omega_{s} \doteq 2 \Gamma(s / 2)^{-1} \sqrt{\pi^{s}} .
$$


Collecting the results from (6.8b), (6.9b) and (6.10) one gets for the complete integral

$$
\begin{aligned}
& \int_{\mathbb{R}^{s}} d^{s} x\left\|E\left(\Delta^{\prime}(\Delta, \Gamma)\right)\left[\alpha_{x}\left(L_{0}\right), L_{0}{ }^{*}\right] E\left(\Delta^{\prime}(\Delta, \Gamma)\right)\right\| \\
& \leqslant \omega_{s} R^{s}\left(a^{\prime}(s) Q^{2 s}\left(L_{0}\right)^{2} R^{-4 s}+b^{\prime}(s)\left\|L_{0}\right\| Q^{2 s}\left(L_{0}\right) R^{-2 s}\right. \\
& \left.+c^{\prime}(s)\left\|E\left(\Delta^{\prime \prime}(\Delta, \Gamma)\right) L_{0} E\left(\Delta^{\prime \prime}(\Delta, \Gamma)\right)\right\|^{2}\right)
\end{aligned}
$$

with suitable $s$-dependent factors. So far the value of $R$ has been left open. To get the concise formula (6.7) we deliberately choose

$$
R^{2 s} \doteq\left\|E\left(\Delta^{\prime \prime}(\Delta, \Gamma)\right) L_{0} E\left(\Delta^{\prime \prime}(\Delta, \Gamma)\right)\right\|^{-2} Q^{2 s}\left(L_{0}\right),
$$

so that (6.11) simplifies to

$$
\begin{aligned}
& \int_{\mathbb{R}^{s}} d^{s} x\left\|E\left(\Delta^{\prime}(\Delta, \Gamma)\right)\left[\alpha_{x}\left(L_{0}\right), L_{0}{ }^{*}\right] E\left(\Delta^{\prime}(\Delta, \Gamma)\right)\right\| \\
& \quad \leqslant \omega_{s} R^{s}\left\|E\left(\Delta^{\prime \prime}(\Delta, \Gamma)\right) L_{0} E\left(\Delta^{\prime \prime}(\Delta, \Gamma)\right)\right\|^{2}\left(a^{\prime}(s)\left\|L_{0}\right\|^{2}+b^{\prime}(s)\left\|L_{0}\right\|+c^{\prime}(s)\right) .
\end{aligned}
$$

Inserting the square root of (6.12) into this estimate and carrying the result over to (6.3), we finally arrive at (6.7), where $\omega_{s}$ has been included in the definition of the coefficients.

Note, that the above argument is independent of the occurrence of $Q^{2 s}\left(L_{0}\right)=0$ or $\left\|E\left(\Delta^{\prime \prime}(\Delta, \Gamma)\right) L_{0} E\left(\Delta^{\prime \prime}(\Delta, \Gamma)\right)\right\|=0$, for in this case $q_{\Delta}\left(L_{0}\right)=0$, so that (6.7) is trivially fulfilled. This consequence is immediate from the norm property of $Q^{2 s}$. As to the second of the above conditions, it turns out to be important that we have included $\Delta \cup(\bar{\Delta}+\Gamma)$ into the definition of $\Delta^{\prime \prime}(\Delta, \Gamma)$. Thence the named assumption implies $\left\|E(\bar{\Delta}+\Gamma) L_{0} E(\Delta)\right\|=\left\|L_{0} E(\Delta)\right\|=0$, and $q_{\Delta}\left(L_{0}\right)=0$ is a result of Lemma 2.11.

Our next aim is to single out a convex subset in the class of all particle weights which turns out to be compact in a suitably chosen topology. To this end it will be assumed from now on that the underlying quantum field theory satisfies the FredenhagenHertel Compactness Criterion, under the assumption of which the following result can be established.

Proposition 6.5. In a quantum field theory which satisfies the Fredenhagen-Hertel Compactness Condition the subsequent mapping, defined for bounded Borel subsets $\Delta$ and compact, convex subsets $\Gamma$ of $\mathbb{R}^{s+1}$,

$$
S_{\Delta}^{\Gamma}: \mathfrak{L}_{0}(\Gamma) \rightarrow \mathfrak{B}(\mathcal{H}) \quad L_{0} \mapsto S_{\Delta}^{\Gamma}\left(L_{0}\right) \doteq L_{0} E(\Delta),
$$

sends balls in $\mathfrak{L}_{0}(\Gamma)$ of finite radius with respect to the norm \|\|\|\|$_{m}, m \in \mathbb{N}$, onto precompact subsets of $\mathfrak{B}(\mathcal{H})$ in its uniform topology.

Proof. Let $\mathfrak{L}_{0, R}^{m}(\Gamma)$ denote the closed $R$-ball, $R>0$, in $\mathfrak{L}_{0}(\Gamma)$ with respect to \|\|$_{\|} \|_{m}$. By Definition 6.2, the condition $\left\|L_{0}\right\|_{m} \leqslant R, L_{0} \in \mathfrak{L}_{0}(\Gamma)$, implies $Q^{m}\left(L_{0}\right)<R$, stating a property of uniform approximation. This means that, given $\varepsilon>0$, there exists a radius 
$r_{0}$, take e. g. $r_{0} \doteq(2 R / \varepsilon)^{-m / 2}$, such that to any $L_{0} \in \mathfrak{L}_{0, R}^{m}(\Gamma)$ we can find a local operator $\left(L_{0}\right)_{r_{0}} \in \mathfrak{A}\left(\mathcal{O}_{r_{0}}\right)$ with

$$
\left\|L_{0}-\left(L_{0}\right)_{r_{0}}\right\|<\varepsilon
$$

Again according to Definition 6.2, one also has $\left\|L_{0}\right\|<R$, so that the collection of local operators just introduced belongs to the closed ball of radius $R+\varepsilon$ in $\mathfrak{A}\left(\mathcal{O}_{r_{0}}\right)$. Now, the Fredenhagen-Hertel Compactness Condition ensues that there exists a finite number of operators $L_{k}, k=1, \ldots, N(\varepsilon)$, in this ball such that any $\left(L_{0}\right)_{r_{0}}$ satisfies the condition

$$
\left\|E(\bar{\Delta}+\Gamma)\left(\left(L_{0}\right)_{r_{0}}-L_{k}\right) E(\Delta)\right\|<\varepsilon
$$

for at least one $k$. Combining this with (6.14a), we see that for any $L_{0} \in \mathfrak{L}_{0, R}^{m}(\Gamma)$ there exists a suitable operator $L_{k} \in \mathfrak{A}\left(\mathcal{O}_{r_{0}}\right)$ with

$$
\left\|E(\bar{\Delta}+\Gamma)\left(L_{0}-L_{k}\right) E(\Delta)\right\| \leqslant\left\|L_{0}-\left(L_{0}\right)_{r_{0}}\right\|+\left\|E(\bar{\Delta}+\Gamma)\left(\left(L_{0}\right)_{r_{0}}-L_{k}\right) E(\Delta)\right\|<2 \varepsilon .
$$

It is an immediate consequence that finitely many elements from $\mathfrak{L}_{0, R}^{m}(\Gamma)$ can be selected, serving as centres of $4 \varepsilon$-balls which cover the set

$$
E(\bar{\Delta}+\Gamma) \mathfrak{L}_{0, R}^{m}(\Gamma) E(\Delta)=\mathfrak{L}_{0, R}^{m}(\Gamma) E(\Delta) .
$$

By arbitrariness of $\varepsilon$, we have thus established precompactness of the mapping $S_{\Delta}^{\Gamma}$ in the sense of the Proposition.

The results presented thus far have only laid down the groundwork for the topological considerations concerning the set of particle weights proper. For the moment we return here to the special continuity properties of the asymptotic functionals resulting from the limiting procedure expounded in Chapter 3. According to Proposition 3.11 in connection with (2.20) of Lemma 2.12, one has for any $L_{1}, L_{2} \in \mathfrak{L}$ and any $A \in \mathfrak{A}$ :

$$
\left|\sigma\left(L_{1}{ }^{*} A L_{2}\right)\right| \leqslant\|h\|_{\infty}\left\|E\left(\bar{\Delta}+\Gamma_{1}\right) A E\left(\bar{\Delta}+\Gamma_{2}\right)\right\| q_{\Delta}\left(L_{1}\right) q_{\Delta}\left(L_{2}\right) .
$$

Specializing now to operators $A$ from the unit ball of a local $C^{*}$-algebra and to vacuum annihilation operators $L_{1}$ and $L_{2}$ from the \|\|$\|_{2 s}$-unit balls of $\mathfrak{L}_{0}\left(\Gamma_{1}\right)$ and $\mathfrak{L}_{0}\left(\Gamma_{2}\right)$, respectively, with compact and convex $\Gamma_{k}$, we infer from (6.15) by use of Lemma 6.4 in connection with Definition 6.2 that there exist constants $C^{\prime}\left(\Delta, \Gamma_{1}\right)$ and $C^{\prime}\left(\Delta, \Gamma_{2}\right)$ such that

$$
\begin{aligned}
\left|\sigma\left(L_{1}{ }^{*} A L_{2}\right)\right| & \leqslant\|h\|_{\infty} C^{\prime}\left(\Delta, \Gamma_{1}\right) C^{\prime}\left(\Delta, \Gamma_{2}\right) . \\
\cdot & \left\|E\left(\bar{\Delta}+\Gamma_{1}\right) A E\left(\bar{\Delta}+\Gamma_{2}\right)\right\|\left\|L_{1} E\left(\Delta^{\prime \prime}\left(\Delta, \Gamma_{1}\right)\right)\right\|^{1 / 2}\left\|L_{2} E\left(\Delta^{\prime \prime}\left(\Delta, \Gamma_{2}\right)\right)\right\|^{1 / 2}
\end{aligned}
$$

with appropriate bounded Borel sets $\Delta^{\prime \prime}\left(\Delta, \Gamma_{1}\right)$ and $\Delta^{\prime \prime}\left(\Delta, \Gamma_{1}\right)$, depending on $\Delta$ and both of the compact sets $\Gamma_{1}$ and $\Gamma_{2}$. An inequality of type (6.16) can likewise be imposed on the corresponding sesquilinear form, which opens up the way to distinguish a certain subclass in the space $S$ of all sesquilinear forms on $\mathfrak{L} \times \mathfrak{L}$. 
Definition 6.6. The set $S^{b}$ of all sesquilinear forms $W(. \mid$. $)$ on $\mathfrak{L} \times \mathfrak{L}$ which are characterized by the existence of constants $C^{\prime}\left(\Delta, \Gamma_{1}\right), C^{\prime}\left(\Delta, \Gamma_{2}\right)$ and $C^{\prime \prime}$ such that the following condition (6.17) holds for any operator $A \in \mathfrak{A}_{1}(\mathcal{O})$ as well as for $L_{1} \in \mathfrak{L}_{0,1}^{2 s}\left(\Gamma_{1}\right)$ and $L_{2} \in \mathfrak{L}_{0,1}^{2 s}\left(\Gamma_{2}\right)$ with compact and convex $\Gamma_{l}$ is a subspace of $S$ :

$$
\begin{aligned}
& \left|W\left(L_{1} \mid A L_{2}\right)\right| \leqslant C^{\prime \prime} C^{\prime}\left(\Delta, \Gamma_{1}\right) C^{\prime}\left(\Delta, \Gamma_{2}\right) . \\
& \cdot\left\|E\left(\bar{\Delta}+\Gamma_{1}\right) A E\left(\bar{\Delta}+\Gamma_{2}\right)\right\|\left\|L_{1} E\left(\Delta^{\prime \prime}\left(\Delta, \Gamma_{1}\right)\right)\right\|^{1 / 2}\left\|L_{2} E\left(\Delta^{\prime \prime}\left(\Delta, \Gamma_{2}\right)\right)\right\|^{1 / 2} .
\end{aligned}
$$

Its intersection with the positive cone $W$ of all particle weights according to Definition 3.14 is again a positive proper convex cone, denoted $W^{b}$, which obviously comprises the particle weights induced by asymptotic functionals.

Due to (6.17), the space $S^{b}$ can be furnished with various seminorm topologies.

Definition 6.7. For any combination of bounded regions $\mathcal{O}$ with compact and convex $\Gamma_{1}$ and $\Gamma_{2}$, a seminorm $\mathcal{P}_{\Gamma_{1}, \Gamma_{2}}^{\mathcal{O}}$ can be introduced on $S^{b}$ by

$$
\mathcal{P}_{\Gamma_{1}, \Gamma_{2}}^{\mathcal{O}}(W(. \mid .)) \doteq \sup \left\{\left|W\left(L_{1} \mid A L_{2}\right)\right|: A \in \mathfrak{A}_{1}(\mathcal{O}), L_{1} \in \mathfrak{L}_{0,1}^{2 s}\left(\Gamma_{1}\right), L_{2} \in \mathfrak{L}_{0,1}^{2 s}\left(\Gamma_{2}\right)\right\} .
$$

The convex subset of $\mathrm{W}^{b}$, which is to be used from now on and will turn out to be compact when furnished with a suitable topology, is introduced again in view of (6.17).

Definition 6.8. $W_{c}^{b}$ is the convex set of all particle weights in $W^{b}$ which satisfy the inequality

$$
\begin{aligned}
& \left|W\left(L_{1} \mid A L_{2}\right)\right| \leqslant C^{\prime}\left(\Delta, \Gamma_{1}\right) C^{\prime}\left(\Delta, \Gamma_{2}\right) \cdot \\
& \quad\left\|E\left(\bar{\Delta}+\Gamma_{1}\right) A E\left(\bar{\Delta}+\Gamma_{2}\right)\right\|\left\|L_{1} E\left(\Delta^{\prime \prime}\left(\Delta, \Gamma_{1}\right)\right)\right\|^{1 / 2}\left\|L_{2} E\left(\Delta^{\prime \prime}\left(\Delta, \Gamma_{2}\right)\right)\right\|^{1 / 2}
\end{aligned}
$$

for all bounded regions $\mathcal{O}$ and all compact and convex $\Gamma_{1}$ and $\Gamma_{2}$. The difference between this condition and (6.17) is that the only $W$-dependent constant $C^{\prime \prime}$ has been omitted.

Remark. According to (6.16), all particle weights arising from asymptotic functionals with $\|h\|_{\infty} \leqslant 1$ satisfy (6.19) and are thus contained in $W_{c}^{b}$.

Now, as a consequence of the Compactness Criterion of Fredenhagen and Hertel, we know that there exist in each case finitely many operators in $\mathfrak{A}_{1}(\mathcal{O})$ as well as in $\mathfrak{L}_{0,1}^{2 s}\left(\Gamma_{l}\right), l=1,2$, serving as centres of $\delta$-balls to cover the sets $\mathfrak{L}_{0,1}^{2 s}\left(\Gamma_{l}\right) E\left(\Delta^{\prime \prime}\left(\Delta, \Gamma_{l}\right)\right)$ and $E\left(\bar{\Delta}+\Gamma_{1}\right) \mathfrak{A}_{1}(\mathcal{O}) E\left(\bar{\Delta}+\Gamma_{2}\right)$. These operators can be used to span finite-dimensional subspaces in $\mathfrak{L}_{0}\left(\Gamma_{1}\right)$ and $\mathfrak{A}(\mathcal{O}) \mathfrak{L}_{0}\left(\Gamma_{2}\right)$. The corresponding space of sesquilinear forms defined on these domains is again finite-dimensional, so that its unit ball with respect to the relative $\mathcal{P}_{\Gamma_{1}, \Gamma_{2}}^{\mathcal{O}}$-topology can be covered by a finite number of $\varepsilon$-balls ( note that bounded sets in finite-dimensional vector spaces are relatively compact). The restriction of any element $W$ of $W_{c}^{b}$ to the named subspaces of $\mathfrak{L}_{0}\left(\Gamma_{1}\right)$ and $\mathfrak{A}(\mathcal{O}) \mathfrak{L}_{0}\left(\Gamma_{2}\right)$ is thus contained in one of these balls. This in turn means, that we can even select a finite number of elements $W_{k} \in W_{c}^{b}, k=1, \ldots, N(2 \varepsilon)$, such that any element of $W_{c}^{b}$ is 
contained in a $2 \varepsilon$-ball around at least one of these chosen forms with respect to the aforementioned relative $\mathcal{P}_{\Gamma_{1}, \Gamma_{2}}^{\mathcal{O}}$-topology. But then it can be shown that the $3 \varepsilon$-balls with respect to the $\mathcal{P}_{\Gamma_{1}, \Gamma_{2}}^{\mathcal{O}}$-topology proper indeed cover all of $W_{c}^{b}$. To see this, let $W_{K}$ be the element pertaining to $W \in W_{c}^{b}$ and let $L_{l} \in \mathfrak{L}_{0,1}^{2 s}\left(\Gamma_{l}\right), l=1,2$, and $A \in \mathfrak{A}_{1}(\mathcal{O})$ be arbitrary. Then there exist operators $L_{l}^{\delta} \in \mathfrak{L}_{0,1}^{2 s}\left(\Gamma_{l}\right)$ and $A^{\delta} \in \mathfrak{A}_{1}(\mathcal{O})$ which satisfy

$$
\begin{array}{r}
\left\|\left(L_{l}-L_{l}^{\delta}\right) E\left(\Delta^{\prime \prime}\left(\Delta, \Gamma_{l}\right)\right)\right\|<\delta, \\
\left\|E\left(\bar{\Delta}+\Gamma_{1}\right)\left(A-A^{\delta}\right) E\left(\bar{\Delta}+\Gamma_{2}\right)\right\|<\delta .
\end{array}
$$

Now, making use of condition (6.19) on elements of $W_{c}^{b}$ in connection with (6.20) as well as of the defining property for $W_{K}$, we get

$$
\begin{aligned}
\left|W\left(L_{1} \mid A L_{2}\right)-W_{K}\left(L_{1} \mid A L_{2}\right)\right| \leqslant\left|W\left(L_{1} \mid A L_{2}\right)-W\left(L_{1}^{\delta} \mid A^{\delta} L_{2}^{\delta}\right)\right| & \\
+\left|W\left(L_{1}^{\delta} \mid A^{\delta} L_{2}^{\delta}\right)-W_{K}\left(L_{1}^{\delta} \mid A^{\delta} L_{2}^{\delta}\right)\right|+\mid W_{K}\left(L_{1}^{\delta} \mid A^{\delta} L_{2}^{\delta}\right) & -W_{K}\left(L_{1} \mid A L_{2}\right) \mid \\
& <2\left(2 \delta^{1 / 2}\right)+2 \varepsilon
\end{aligned}
$$

Since we are free to choose $\delta$ appropriately small in dependence on a given $\varepsilon>0$, this final relation shows, upon taking the supremum with respect to the operators appearing on the left-hand side, that for each $W \in W_{c}^{b}$ there exists at least one $W_{K} \in W_{c}^{b}$ such that $\mathcal{P}_{\Gamma_{1}, \Gamma_{2}}^{\mathcal{O}}\left(W-W_{K}\right)<3 \varepsilon$ in accordance with our statement.

As in Chapter 4 we want to pass at this point to countable families $\left\{\mathcal{O}_{n}\right\}_{n \in \mathbb{N}}$ and $\left\{\Gamma_{l}\right\}_{l \in \mathbb{N}}$ of bounded regions in space-time and of compact and convex subsets of $\complement \bar{V}_{+}$. By Definition 6.7 any triple taken from these sequences defines a seminorm on $S^{b}$. In this way $S^{b}$ can be topologized with a sequence $\left\{\mathcal{P}_{m}\right\}_{m \in \mathbb{N}}$ of seminorms and thereby becomes a locally convex (Hausdorff) space. This space is metrizable according to [44, Chapter Four, $\S 18,2$.(2)] and its topology can moreover be derived from the increasing sequence of seminorms

$$
\mathcal{R}^{k}(W) \doteq \max _{1 \leqslant m \leqslant k} \mathcal{P}_{m}(W), \quad W \in \mathrm{S}^{b}, \quad k \in \mathbb{N} .
$$

This countable system can then be used to define an (F)-norm [44, p. 163] on $S^{b}$ which furnishes this space with the same topology. It is given by

$$
\|W\|_{F} \doteq \sum_{k=1}^{\infty} \frac{1}{2^{k}} \frac{\mathcal{R}^{k}(W)}{1+\mathcal{R}^{k}(W)}, \quad W \in S^{b},
$$

and generates a translation-invariant metric [44, Chapter Four, § 18, 2.(3)]. Given $\varepsilon>0$ there exists, according to (6.23), an index $M$ such that for any $W, W^{\prime} \in \mathrm{S}^{b}$

$$
\begin{aligned}
\left\|W-W^{\prime}\right\|_{F}= & \sum_{k=1}^{\infty} \frac{1}{2^{k}} \frac{\mathcal{R}^{k}\left(W-W^{\prime}\right)}{1+\mathcal{R}^{k}\left(W-W^{\prime}\right)} \\
& \quad<\frac{\varepsilon}{2}+\sum_{k=1}^{M} \frac{1}{2^{k}} \frac{\mathcal{R}^{k}\left(W-W^{\prime}\right)}{1+\mathcal{R}^{k}\left(W-W^{\prime}\right)} \leqslant \frac{\varepsilon}{2}+\sum_{k=1}^{M} \frac{1}{2^{k}} \cdot \mathcal{R}^{M}\left(W-W^{\prime}\right) .
\end{aligned}
$$

A consequence of the preceding paragraph in combination with the definition (6.22) is the fact that $W_{c}^{b}$ can be covered by a finite number of balls with a given arbitrarily 
small radius with respect to the $\mathcal{R}^{k}$-topologies. It is then an immediate conclusion from the definitions involved that the sesquilinear functionals arising as limits with respect to $\|.\|_{F}$ of sequences in $W_{c}^{b}$ are again elements of $W_{c}^{b}$. This convex subset thus turns out to be closed. Since it has been seen above to be precompact, it is indeed compact in the $\|\cdot\|_{F}$-topology.

Proposition 6.9. The convex set $\mathrm{W}_{c}^{b}$ in the class of all particle weights is compact with respect to the metric derived from the $(F)$-norm $\|\mid\| \|_{F}$.

The above work has laid the foundation for an application of Choquet's Theorem [1, Corollary I.4.9] which tells us that any particle weight $\langle. \mid$.$\rangle in the metrizable compact$ convex set $W_{c}^{b}$ can be represented by a positive and normalized measure vanishing off its extreme boundary $\partial_{e} W_{c}^{b}$ :

$$
\langle\cdot \mid \cdot\rangle=\int_{\partial_{e} W_{c}^{b}} d v(\zeta)\langle\cdot \mid \cdot\rangle_{\zeta}
$$

The above result represents the present status of the Choquet approach to a disintegration theory for particle weights. The problem to be tackled at this point is the open question of how a base can be fitted into the cone $W^{b}$ which is completely contained in $W_{c}^{b}$. This would allow for a disintegration of a particle weight on this base in terms of extremal points, defining extremal rays of the cone $W^{b}$ and representing pure particle weights. On this foundation a complete theory in parallel to that developed for states on a $C^{*}$-algebra $\mathfrak{A}$ in [11, Sections 4.1 and 4.2] still awaits its completion. The advantage of this approach in comparison to the spatial disintegration presented in Chapter 4 is that, apart from the somewhat intricate topological considerations, it is more direct and the resulting pure particle weights are no longer subject to the restrictive Definition 4.1. On the other hand, the mathematical problems concerning convex sets in infinite-dimensional spaces are far from being trivial. Therefore, a lot of work remains to be done until eventually the particle content of a quantum field theory is seen to be encoded in the geometrical structure (the set of extreme rays) of a positive cone of particle weights. 


\section{Chapter 7}

\section{Summary and Outlook}

The present work is based on the general point of view that the concept of 'particles' is asymptotic in nature and simultaneously has to be founded by making appropriate use of the notion of locality. This reflects our conviction that the long-standing problem of 'asymptotic completeness' of quantum field theory, i.e., the question if a quantum field theoretic model can be interpreted completely in terms of particles, has to be tackled by the aid of further restrictions on the general structure, which essentially are of a local character. The question is, what the local structure of a theory should be in order that it governs scattering processes in such a way that asymptotically the physical states appear to clot in terms of certain entities named particles. The compactness and nuclearity conditions discussed in [18] and the references therein are examples of this kind of approach. We do not claim that they already give a complete answer, but believe that they indicate the right direction.

In this thesis we have constructed asymptotic functionals on a certain algebra of detectors giving rise to particle weights which can be interpreted as mixtures of particle states. A disintegration theory has been developed for restricted particle weights by means of a highly technical procedure in Chapter 4. This constitutes the basis for the definition of mass and spin even in the case of charged states [17]. We are convinced that the technicalities involved can be dissolved by future research. In this connection the analysis of concrete models may be helpful. Such investigations are already under way. They concern the Schwinger model [30] and an application of our formalism to quantum electrodynamics [27]. It is expected that some insight may be gained with respect to the open questions mentioned in the various chapters. E. g., the convergence problem in connection with Theorem 3.10 can perhaps be solved with additional information at hand, and the direct integral decomposition of Chapter 4 might get more manageable, unfolding the connection between the intrinsic energy-momenta pertaining to the irreducible representations and the geometrical energy-momenta (velocities) that stem from the asymptotic functionals.

So far we have considered single particle weights. Another field of future research is the inspection of coincidence arrangements of detectors as in [3]. In this respect, too, the analysis of concrete models is helpful.

As indicated by Chapter 5 and in view of the partial results presented in Chapter 6 phase space restrictions seem to be a key ingredient in the general analysis, in particular 
of the Choquet approach to disintegration. This theory is still in its initial stage. But, difficult as the mathematical problems concerning convex sets in infinite-dimensional spaces are, it deserves further efforts. Presumably, both the spatial disintegration and the Choquet decomposition will eventually turn out to be essentially equivalent, revealing relations similar to those encountered in the disintegration theory of states on $C^{*}$-algebras [11, Chapter 4]. Further studies have to disclose the geometrical structure of the positive cone of particle weights, as the particle content of a theory seems to be encoded in this kind of information. 


\section{Appendix A}

\section{Concepts of Differentiability}

Various notions of differentiability have to be used in this work and some of them take on a somewhat unusual shape. So it seems right to collect in this appendix a number of definitions and propositions, both to assign a precise meaning to the concepts proper and to their consequences as well as to fix the notation.

\section{A.1 Differentiation in Locally Convex Spaces}

Definition A.1. Let $\mathfrak{X}$ be a (real or complex) normed space and let $\mathfrak{V}$ be a locally convex space over the same field whose topology is defined by the family $\left\{q_{\lambda}: \lambda \in L\right\}$ of seminorms which separate the points of $\mathfrak{V}$. Suppose further that we are given an open subset $\mathfrak{G}$ of $\mathfrak{X}$.

(a) A mapping $F: \mathfrak{G} \rightarrow \mathfrak{V}$ is called differentiable at the point $\mathfrak{x} \in \mathfrak{G}$ if there exists a continuous linear mapping $T: \mathfrak{X} \rightarrow \mathfrak{V}$ such that for any vector $\mathfrak{h}$ in a certain $\mathfrak{o}$ neighbourhood $\mathfrak{U} \subseteq \mathfrak{X}$ the increment $F(\mathfrak{x}+\mathfrak{h})-F(\mathfrak{x})$ allows for the linearized approximation

$$
F(\mathfrak{x}+\mathfrak{h})-F(\mathfrak{x})=T \mathfrak{h}+R[F, \mathfrak{x}](\mathfrak{h}),
$$

where $R[F, \mathfrak{x}]$ is a mapping on $\mathfrak{U}$ to $\mathfrak{V}$ subject to the condition

$$
\lim _{\mathfrak{h} \rightarrow \mathfrak{0}}\|\mathfrak{h}\|^{-1} q_{\lambda}(R[F, \mathfrak{x}](\mathfrak{h}))=0
$$

for any seminorm $q_{\lambda}, \lambda \in L$. The linear operator $T$ occurring in (A.1a) is signified as $\mathfrak{D} F(\mathfrak{x})$ and called the derivative of $F$ at $\mathfrak{x}$.

(b) The mapping $F: \mathfrak{G} \rightarrow \mathfrak{V}$ is called differentiable if it is differentiable at any $\mathfrak{x} \in \mathfrak{G}$.

(c) The differentiable mapping $F: \mathfrak{G} \rightarrow \mathfrak{V}$ is called continuously differentiable if the mapping $\mathfrak{G} \ni \mathfrak{x} \mapsto \mathfrak{D} F(\mathfrak{x}) \mathfrak{h} \in \mathfrak{V}$, which exists by assumption, is continuous with respect to the locally convex topology of $\mathfrak{V}$ for any given $\mathfrak{h} \in \mathfrak{X}$.

Remark. The definition of the continuous linear operator $\mathfrak{D} F(\mathfrak{x})$ requires uniqueness of the corresponding $T$ in (A.1a), but this is easily established. Assume the existence of another o-neighbourhood $\mathfrak{U}^{\prime}$, a continuous linear operator $T^{\prime}: \mathfrak{X} \rightarrow \mathfrak{V}$ and a mapping 
$R^{\prime}[F, \mathfrak{x}]: \mathfrak{U}^{\prime} \rightarrow \mathfrak{V}$ which, upon insertion into (A.1a), represent the increment $F(\mathfrak{x}+\mathfrak{h})-$ $F(\mathfrak{x})$ such that $R^{\prime}[F, \mathfrak{x}]$ fulfills a condition analogous to (A.1b). Then

$$
T \mathfrak{h}-T^{\prime} \mathfrak{h}=R^{\prime}[F, \mathfrak{x}](\mathfrak{h})-R[F, \mathfrak{x}](\mathfrak{h}), \quad \mathfrak{h} \in \mathfrak{U} \cap \mathfrak{U}^{\prime} .
$$

Let $\mathfrak{y} \in \mathfrak{X}, \mathfrak{y} \neq \mathfrak{o}$, be arbitrary but fixed, then for $\alpha \in \mathbb{C} \backslash\{0\}$ small enough we infer from the above equation due to the linearity of both $T$ and $T^{\prime}$

$$
\begin{aligned}
q_{\lambda}\left(T \mathfrak{y}-T^{\prime} \mathfrak{y}\right)=q_{\lambda}\left(\alpha^{-1}\left(R^{\prime}[F, \mathfrak{x}](\alpha \mathfrak{y})-R[F, \mathfrak{x}](\alpha \mathfrak{y})\right)\right) \\
=\|\mathfrak{y}\|\|\alpha \mathfrak{y}\|^{-1} q_{\lambda}\left(R^{\prime}[F, \mathfrak{x}](\alpha \mathfrak{y})-R[F, \mathfrak{x}](\alpha \mathfrak{y})\right),
\end{aligned}
$$

where the right-hand side vanishes in the limit $\alpha \rightarrow 0$ for any seminorm $q_{\lambda}$, according to (A.1b). This yields $q_{\lambda}(T \mathfrak{y})=q_{\lambda}\left(T^{\prime} \mathfrak{y}\right)$, valid also for $\mathfrak{y}=\mathfrak{o}$, and as a consequence $T \mathfrak{y}=T^{\prime} \mathfrak{y}$ for any $\mathfrak{y} \in \mathfrak{X}$ since the seminorms $q_{\lambda}$ separate the points in $\mathfrak{V}$.

An immediate consequence of the presumed continuity of the linear operators $\mathfrak{D F}(\mathfrak{x})$, entering as derivatives the representation (A.1a) of the increment of $F$ at $\mathfrak{x}$, is the fact that differentiability implies continuity.

Corollary A.2. Let $\mathfrak{X}$ be a normed space and let $\mathfrak{V}$ be a locally convex space. If the mapping $F: \mathfrak{G} \rightarrow \mathfrak{V}, \mathfrak{G} \subseteq \mathfrak{X}$ open, is differentiable at the point $\mathfrak{x} \in \mathfrak{G}$ then it is also continuous in $\mathfrak{x}$.

The methods used in the standard theory of differentiable functions yield the following propositions when applied to the concept laid open in Definition A.1, the main modification being the occurrence of seminorms $q_{\lambda}$ on $\mathfrak{V}$ in (A.1b).

Proposition A.3 (Product Rule for Derivatives). Let $\mathfrak{X}$ be a normed space and $\mathfrak{G}$ an open subset of $\mathfrak{X}$.

(i) Suppose that $\mathfrak{V}$ is a locally convex space and that the mappings $F: \mathfrak{G} \rightarrow \mathfrak{V}$ and $f: \mathfrak{G} \rightarrow \mathbb{K}, \mathbb{K}$ the scalar field of both $\mathfrak{X}$ and $\mathfrak{V}$, are differentiable at $\mathfrak{x} \in \mathfrak{G}$. Then their product $f F$ is differentiable at this point, too, and the derivative at $\mathfrak{x}$ is given by

$$
\mathfrak{D}(f F)(\mathfrak{x}) \mathfrak{h}=\mathfrak{D} f(\mathfrak{x}) \mathfrak{h} F(\mathfrak{x})+f(\mathfrak{x}) \mathfrak{D} F(\mathfrak{x}) \mathfrak{h}, \quad \mathfrak{h} \in \mathfrak{X} .
$$

(ii) Let $\mathfrak{Y}$ be a normed algebra and assume that the mappings $F: \mathfrak{G} \rightarrow \mathfrak{Y}$ and $G: \mathfrak{G} \rightarrow$ $\mathfrak{Y}$ are differentiable at $\mathfrak{x} \in \mathfrak{G}$. Then their product $F G$ is differentiable at $\mathfrak{x}$, too, and the derivative is

$$
\mathfrak{D}(F G)(\mathfrak{x}) \mathfrak{h}=\mathfrak{D} F(\mathfrak{x}) \mathfrak{h} G(\mathfrak{x})+F(\mathfrak{x}) \mathfrak{D} G(\mathfrak{x}) \mathfrak{h}, \quad \mathfrak{h} \in \mathfrak{X}
$$

Proposition A.4 (Chain Rule for Derivatives). Let $\mathfrak{X}$ and $\mathfrak{Y}$ be normed spaces and let $\mathfrak{V}$ be a locally convex space. Assume further that the mapping $G: \mathfrak{G}_{1} \rightarrow \mathfrak{Y}$ is differentiable at $\mathfrak{x} \in \mathfrak{G}_{1}$ and that the mapping $F: \mathfrak{G}_{2} \rightarrow \mathfrak{V}$ is differentiable at $G(\mathfrak{x})$, where $\mathfrak{G}_{1}$ and $\mathfrak{G}_{2}$ are open subsets of $\mathfrak{X}$ and $\mathfrak{Y}$, respectively, and $G\left(\mathfrak{G}_{1}\right) \subseteq \mathfrak{G}_{2}$. Then the composition of $F$ and $G: F \circ G: \mathfrak{G}_{1} \rightarrow \mathfrak{V}$, exists and is differentiable at $\mathfrak{x}$ with a derivative connected to those of $F$ and $G$ through

$$
\mathfrak{D}(F \circ G)(\mathfrak{x})=\mathfrak{D} G(F(\mathfrak{x})) \circ \mathfrak{D} F(\mathfrak{x}) .
$$


The fundamental Mean Value Theorem which has to be formulated in the setting of Definition A.1 is based on the following two lemmas. Their proof as well as that of the theorem proper is an adaptation of the reasoning to be found in [37, Kapitel XX, Abschnitt 175].

Lemma A.5. Let $F:[a, b] \rightarrow \mathfrak{V}$ be a continuous mapping on the compact interval $[a, b] \subseteq \mathbb{R}$ to the locally convex space $\mathfrak{V}$ and suppose that it is differentiable on the interior of this set with $\mathfrak{D} F(x)=0$ for any $x \in] a, b[$. Then $F$ is constant on $[a, b]$.

Proof. Let $s$ and $t$ be arbitrary distinct points in $] a, b[$. We shall assume $s<t$ and want to show that $F(s)=F(t)$. Define $u \doteq 2^{-1}(t-s)$ and consider one of the seminorms $q_{\lambda}$ topologizing $\mathfrak{V}$. There are two possibilities:

$$
\begin{aligned}
& q_{\lambda}(F(u)-F(s)) \geqslant q_{\lambda}(F(t)-F(u)), \\
& q_{\lambda}(F(t)-F(u))>q_{\lambda}(F(u)-F(s)) .
\end{aligned}
$$

Depending on the actual situation we define an interval $] s_{1}, t_{1}\left[\subseteq[a, b]\right.$, choosing $s_{1} \doteq s$, $t_{1} \doteq u$ in case (A.2a) and $s_{1} \doteq u, t_{1} \doteq t$ in case (A.2b). Independent of this selection is the estimate

$$
q_{\lambda}(F(t)-F(s)) \leqslant q_{\lambda}(F(t)-F(u))+q_{\lambda}(F(u)-F(s)) \leqslant 2 q_{\lambda}\left(F\left(t_{1}\right)-F\left(s_{1}\right)\right) .
$$

The same procedure can then be applied to the interval $] s_{1}, t_{1}[$, to the resulting interval ]$s_{2}, t_{2}$ [ and so on. In this way a sequence of intervals $] s_{n}, t_{n}$ [ is constructed, which is decreasing with respect to the inclusion relation: $] s_{n+1}, t_{n+1}[\subseteq] s_{n}, t_{n}[$. Furthermore the lengths are explicitly known as $t_{n}-s_{n}=2^{-n}(t-s)$ and the estimate (A.3) can be generalized to

$$
q_{\lambda}(F(t)-F(s)) \leqslant 2^{n} q_{\lambda}\left(F\left(t_{n}\right)-F\left(s_{n}\right)\right) .
$$

There exists exactly one point $\left.u_{0} \in\right] a, b[$ belonging to all intervals of this sequence and by assumption $\mathfrak{D} F\left(u_{0}\right)=0$, so that for $h$ in a small 0-neighbourhood $\mathcal{U} \subseteq \mathbb{R}$ the increment of $F$ at $u_{0}$ is represented by

$$
F\left(u_{0}+h\right)-F\left(u_{0}\right)=h R(h)
$$

with a mapping $R: \mathcal{U} \rightarrow \mathfrak{V}$ satisfying

$$
\lim _{h \rightarrow 0} q_{\lambda}(R(h))=0 .
$$

Hence, given $\varepsilon>0$, there exists $N \in \mathbb{N}$ such that $q_{\lambda}\left(R\left(u_{0}-s_{n}\right)\right)$ and $q_{\lambda}\left(R\left(t_{n}-u_{0}\right)\right)$ are majorized by $(t-s)^{-1} \varepsilon$ for $n>N$. According to (A.5a) this implies

$$
\begin{aligned}
& q_{\lambda}\left(F\left(t_{n}\right)-F\left(s_{n}\right)\right) \leqslant q_{\lambda}\left(F\left(t_{n}\right)-F\left(u_{0}\right)\right)+q_{\lambda}\left(F\left(s_{n}\right)-F\left(u_{0}\right)\right) \\
& \leqslant\left|t_{n}-u_{0}\right| q_{\lambda}\left(R\left(t_{n}-u_{0}\right)\right)+\left|u_{0}-s_{n}\right| q_{\lambda}\left(R\left(u_{0}-s_{n}\right)\right) \\
& \quad \leqslant\left(t_{n}-u_{0}\right) \frac{\varepsilon}{t-s}+\left(u_{0}-s_{n}\right) \frac{\varepsilon}{t-s}=\left(t_{n}-s_{n}\right) \frac{\varepsilon}{t-s}=\frac{\varepsilon}{2^{n}},
\end{aligned}
$$


where we made use of the length formula for the interval $] s_{n}, t_{n}[$. From (A.4) one then infers

$$
q_{\lambda}(F(t)-F(s)) \leqslant 2^{n} \frac{\varepsilon}{2^{n}}=\varepsilon,
$$

so that, by arbitrariness of $\varepsilon$ and $q_{\lambda}$ together with the separation property of the seminorms, we see that $F(t)=F(s)=\mathfrak{v}_{0} \in \mathfrak{V}$. This relation holds for any $\left.s, t \in\right] a, b[$ and extends by the supposed continuity of $F$ to all of $[a, b]$, establishing $F \equiv \mathfrak{v}_{0}$ as stated.

Lemma A.6. Let $F:[a, b] \rightarrow \mathfrak{V}$ be a continuous mapping on the compact interval $[a, b] \subseteq \mathbb{R}$ to the locally convex space $\mathfrak{V}$ and define $G:[a, b] \rightarrow \overline{\mathfrak{V}}, \overline{\mathfrak{V}}$ the completion of $\mathfrak{V}$, through the integral

$$
G(x) \doteq \int_{a}^{x} d \vartheta F(\vartheta), \quad x \in[a, b]
$$

Then the mapping $G$ is differentiable for any $\left.x_{0} \in\right] a, b[$ and the action of the derivative $\mathfrak{D} G\left(x_{0}\right)$ as a linear operator on $\mathbb{R}$ is given by

$$
\mathfrak{D} G\left(x_{0}\right) h=h F\left(x_{0}\right), \quad h \in \mathbb{R} .
$$

Proof. By [26, II.6.2] $G$ is a well-defined $\overline{\mathfrak{V}}$-valued mapping on the compact interval $[a, b]$. For $\left.x_{0} \in\right] a, b\left[\right.$ and $h \in \mathbb{R}$ satisfying $x_{0}+h \in[a, b]$ we have

$$
G\left(x_{0}+h\right)-G\left(x_{0}\right)=\int_{x_{0}}^{x_{0}+h} d \vartheta F(\vartheta)
$$

hence

$$
G\left(x_{0}+h\right)-G\left(x_{0}\right)-h F\left(x_{0}\right)=\int_{x_{0}}^{x_{0}+h} d \vartheta\left(F(\vartheta)-F\left(x_{0}\right)\right) \doteq \rho(h) .
$$

Now by assumption, $\vartheta \mapsto q_{\lambda}\left(F(\vartheta)-F\left(x_{0}\right)\right)$ is continuous on the compact interval $I_{h}$ of integration for any of the defining seminorms $q_{\lambda}$ of $\mathfrak{V}$, and, according to [26, II.6.2 in connection II.5.4], one has for any $h$ the estimate

$$
|h|^{-1} q_{\lambda}(\rho(h)) \leqslant|h|^{-1}\left|\int_{x_{0}}^{x_{0}+h} d \vartheta q_{\lambda}\left(F(\vartheta)-F\left(x_{0}\right)\right)\right| \leqslant \max _{\vartheta \in I_{h}} q_{\lambda}\left(F(\vartheta)-F\left(x_{0}\right)\right),
$$

where the right-hand side vanishes in the limit $h \rightarrow 0$. Thus (A.7a) corresponds to the representation (A.1a) of Definition A.1 in terms of the increment $G\left(x_{0}+h\right)-G\left(x_{0}\right)$ with a residual term $\rho(h)$ satisfying (A.1b). This proves differentiability of $G$ on $] a, b[$ along with relation (A.6). 
Theorem A.7 (Mean Value Theorem). Let $\mathfrak{X}$ be a normed space and $\mathfrak{V}$ be a locally convex space. Let furthermore $F: \mathfrak{G} \rightarrow \mathfrak{V}, \mathfrak{G} \subseteq \mathfrak{X}$ open, be a continuously differentiable mapping (in the sense of Definition A.1) and consider $\mathfrak{x}_{0} \in \mathfrak{G}$ and $\mathfrak{h} \in \mathfrak{X}$ small enough so that $\mathfrak{x}_{0}+\vartheta \mathfrak{h} \in \mathfrak{G}$ for $0 \leqslant \vartheta \leqslant 1$. Then

$$
F\left(\mathfrak{x}_{0}+\mathfrak{h}\right)-F\left(\mathfrak{x}_{0}\right)=\int_{0}^{1} d \vartheta \mathfrak{D} F\left(\mathfrak{x}_{0}+\vartheta \mathfrak{h}\right) \mathfrak{h} .
$$

Proof. Given $\mathfrak{x}_{0} \in \mathfrak{G}$ and $\mathfrak{h} \in \mathfrak{X}$ as above we define two mappings $F_{1}$ and $F_{2}$ on the compact interval $[0,1]$ to $\mathfrak{V}$ respectively $\overline{\mathfrak{V}}$ through

$$
\begin{aligned}
s & \mapsto F_{1}(s) \doteq F\left(\mathfrak{x}_{0}+s \mathfrak{h}\right), \\
s \mapsto F_{2}(s) & \doteq \int_{0}^{s} d \vartheta \mathfrak{D} F\left(\mathfrak{x}_{0}+\vartheta \mathfrak{h}\right) \mathfrak{h} .
\end{aligned}
$$

From Lemma A.6 and Proposition A.4 we infer $\mathfrak{D} F_{2}(s)=\mathfrak{D} F\left(\mathfrak{x}_{0}+s \mathfrak{h}\right) \mathfrak{h}=\mathfrak{D} F_{1}(s)$ for any $s \in] 0,1\left[\right.$. This implies, according to Lemma A.5, that the mapping $F_{1}-F_{2}$ is constant on the interval $[0,1]$ (Note, that $F_{1}$ as well as $F_{2}$ are continuous.). Hence

$$
F\left(\mathfrak{x}_{0}\right)=F_{1}(0)-F_{2}(0)=F_{1}(1)-F_{2}(1)=F\left(\mathfrak{x}_{0}+\mathfrak{h}\right)-\int_{0}^{1} d \vartheta \mathfrak{D} F\left(\mathfrak{x}_{0}+\vartheta \mathfrak{h}\right) \mathfrak{h},
$$

which is just equation (A.8) re-written.

\section{A.2 Differentiation on Analytic Manifolds}

Being of a local nature, the concept of differentiability set out in Definition A.1 can be generalized to $\mathfrak{V}$-valued mappings on analytic manifolds in the following way.

Definition A.8. Let $\mathcal{M}$ be a (real or complex) analytic manifold of dimension $d$ and let $\mathfrak{V}$ be a locally convex space over the same field. Let furthermore $(\mathcal{U}, \phi)$ denote a local chart on $\mathcal{M}$, which means that $\phi(\mathcal{U}) \subseteq \mathbb{K}^{d}, \mathbb{K}=\mathbb{R}$ or $\mathbb{K}=\mathbb{C}$.

(a) The mapping $F: \mathcal{U} \rightarrow \mathfrak{V}$ is called differentiable (with respect to $\phi$ ) at $m_{0} \in \mathcal{U}$ if $F \circ \phi^{-1}: \phi(\mathcal{U}) \rightarrow \mathfrak{V}$ is differentiable at $\phi\left(m_{0}\right)$ in the sense of Definition A.1. The derivative is denoted $\mathfrak{D}_{\phi} F\left(m_{0}\right) \doteq \mathfrak{D}\left(F \circ \phi^{-1}\right)\left(\phi\left(m_{0}\right)\right)$.

(b) $F: U \rightarrow \mathfrak{V}$ is called (continuously) differentiable if $F \circ \phi^{-1}$ is (continuously) differentiable in the sense of Definition A.1.

(c) The mapping $F: \mathcal{N} \rightarrow \mathfrak{V}$ is called (continuously) differentiable if to any $m_{0} \in$ $\mathcal{M}$ there exists a local chart $(\mathcal{U}, \phi)$ containing $m_{0}$ such that $F\lceil\mathcal{U}$ is (continuously) differentiable with respect to $\phi$.

(d) Let $\left\{\boldsymbol{e}_{i}: i=1, \ldots, d\right\}$ be the canonical orthonormal basis of $\mathbb{K}^{d}$. Then $F: \mathcal{U} \rightarrow \mathfrak{V}$ is said to have continuous partial derivatives if there exist $d$ continuous mappings $F_{\phi}^{i}$ : $\mathcal{U} \rightarrow \mathfrak{V}$, such that the increment of $F$ in direction $\boldsymbol{e}_{i}$ at any $m_{0}=\phi^{-1}\left(\boldsymbol{t}_{0}\right) \in \mathcal{U}$ allows for the representation

$$
F \circ \phi^{-1}\left(\boldsymbol{t}_{0}+h \boldsymbol{e}_{i}\right)-F \circ \phi^{-1}\left(\boldsymbol{t}_{0}\right)=h F_{\phi}^{i}\left(m_{0}\right)+R\left[F \circ \phi^{-1}, \boldsymbol{t}_{0}\right](h)
$$


if $h \in \mathbb{K}$ is small enough, where the residual term satisfies

$$
\lim _{h \rightarrow 0}|h|^{-1} q_{\lambda}\left(R\left[F \circ \phi^{-1}, t_{0}\right](h)\right)=0
$$

for any seminorm $q_{\lambda}, \lambda \in L$.

(e) Higher derivatives of the mapping $F: \mathcal{U} \rightarrow \mathfrak{V}$ are defined recursively in terms of partial derivatives of the mappings $F_{\phi}^{i}, i=1, \ldots, d$, and, if they happen to exist, are denoted $F_{\phi}^{\kappa}$ for multi-indices $\kappa \doteq\left(k_{1}, \ldots, k_{d}\right) \in \mathbb{N}_{0}^{d}$ in an obvious fashion (for given $i$ let $F_{\phi}^{\kappa_{i}} \doteq F_{\phi}^{i}$ where all entries in $\kappa_{i}$ apart from $k_{i}=1$ vanish). $F: \mathcal{U} \rightarrow \mathfrak{V}$ is called $N$-fold (or infinitely often) continuously differentiable if the mappings $F_{\phi}^{\mathrm{K}}$ exist and are continuous for any $|\kappa| \doteq \sum_{i} k_{i} \leqslant N$ (or $\left.|\kappa|<\infty\right)$. These concepts apply equally to mappings $F$ defined on all of $\mathcal{M}$.

Remark. If $F$ is differentiable at $m_{0} \in \mathcal{U}$ with respect to the local chart $(\mathcal{U}, \phi)$ it is also differentiable with respect to any other local chart $(\mathcal{V}, \psi)$ containing $m_{0}$, and according to Proposition A.4 one has

$$
\mathfrak{D}_{\psi} F\left(m_{0}\right)=\mathfrak{D}_{\phi} F\left(m_{0}\right) \circ\left(\phi \circ \psi^{-1}\right)^{\prime}\left(\psi\left(m_{0}\right)\right)
$$

where $\left(\phi \circ \psi^{-1}\right)^{\prime}$ denotes the first derivative (Jacobi matrix) of the analytic function $\phi \circ \psi^{-1}: \psi(\mathcal{U} \cap \mathcal{V}) \rightarrow \phi(\mathcal{V} \mathcal{\mathcal { V }})$.

Strictly speaking, the definition of and notation for higher derivatives of a mapping $F: U \rightarrow \mathfrak{V}$ is justified only after the following two results are established.

Proposition A.9. $F: U \rightarrow \mathfrak{V}$ is continuously differentiable if and only if it has continuous partial derivatives in all directions $\boldsymbol{e}_{i}, i=1, \ldots, d$.

Proof. (i) If $F$ is continuously differentiable the mappings

$$
\mathcal{U} \ni m_{0} \mapsto F_{\phi}^{i}\left(m_{0}\right) \doteq \mathfrak{D}_{\phi} F\left(m_{0}\right) \boldsymbol{e}_{i}
$$

are continuous for any $i$; furthermore (A.10a) and (A.10b) correspond for each $i$ exactly to (A.1a) and (A.1b) of Definition A.1 setting $\mathfrak{h}=h \boldsymbol{e}_{i}$, so that the first part of the statement is almost trivial.

(ii) Let all the partial derivatives of $F$ exist as continuous mappings $F_{\phi}^{i}: \mathcal{U} \rightarrow \mathfrak{V}$, then, for small $\boldsymbol{h}=\sum_{i} h_{i} \boldsymbol{e}_{i} \in \mathbb{K}^{d}$, we have through an application of the Mean Value Theorm A.7 for any $m_{0}=\phi^{-1}\left(\boldsymbol{t}_{0}\right) \in \mathcal{U}$

$$
\begin{aligned}
& F \circ \phi^{-1}\left(\boldsymbol{t}_{0}+\boldsymbol{h}\right)-F \circ \phi^{-1}\left(\boldsymbol{t}_{0}\right) \\
& \quad=\sum_{i=1}^{d}\left[F \circ \phi^{-1}\left(\boldsymbol{t}_{0}+\sum_{j=1}^{i} h_{j} \boldsymbol{e}_{j}\right)-F \circ \phi^{-1}\left(\boldsymbol{t}_{0}+\sum_{j=1}^{i-1} h_{j} \boldsymbol{e}_{j}\right)\right] \\
& =\sum_{i=1}^{d} h_{i} F_{\phi}^{i}\left(m_{0}\right)+\sum_{i=1}^{d} \int_{0}^{1} d \vartheta h_{i}\left[F_{\phi}^{i} \circ \phi^{-1}\left(\boldsymbol{t}_{0}+\sum_{j=1}^{i-1} h_{j} \boldsymbol{e}_{j}+\vartheta h_{i} \boldsymbol{e}_{i}\right)-F_{\phi}^{i}\left(m_{0}\right)\right] .
\end{aligned}
$$


Due to continuity of the mappings $F_{\phi}^{i}$, the second term on the right-hand side multiplied with $|\boldsymbol{h}|^{-1}$ can be estimated by

$$
\begin{aligned}
|\boldsymbol{h}|^{-1} q_{\lambda}\left(\sum_{i=1}^{d} \int_{0}^{1} d \vartheta h_{i}\left[F_{\phi}^{i} \circ \phi^{-1}\left(\boldsymbol{t}_{0}+\sum_{j=1}^{i-1} h_{j} \boldsymbol{e}_{j}+\vartheta h_{i} \boldsymbol{e}_{i}\right)-F_{\phi}^{i}\left(m_{0}\right)\right]\right) \\
\leqslant|\boldsymbol{h}|^{-1} \sum_{i=1}^{d}\left|h_{i}\right| \max _{0 \leqslant \vartheta \leqslant 1} q_{\lambda}\left(F_{\phi}^{i} \circ \phi^{-1}\left(\boldsymbol{t}_{0}+\sum_{j=1}^{i-1} h_{j} \boldsymbol{e}_{j}+\vartheta h_{i} \boldsymbol{e}_{i}\right)-F_{\phi}^{i}\left(m_{0}\right)\right) \\
\leqslant \sum_{i=1}^{d} \max _{0 \leqslant \vartheta \leqslant 1} q_{\lambda}\left(F_{\phi}^{i} \circ \phi^{-1}\left(\boldsymbol{t}_{0}+\sum_{j=1}^{i-1} h_{j} \boldsymbol{e}_{j}+\vartheta h_{i} \boldsymbol{e}_{i}\right)-F_{\phi}^{i}\left(m_{0}\right)\right),
\end{aligned}
$$

where the last expression of the above inequality is seen to vanish in the limit $\boldsymbol{h} \rightarrow \boldsymbol{O}$ by assumption. Thus (A.13) in connection with (A.14) establishes continuous differentiability of the mapping $F: \mathcal{U} \rightarrow \mathfrak{V}$ with

$$
\mathfrak{D}_{\phi} F\left(m_{0}\right) \boldsymbol{h}=\sum_{i=1}^{d} h_{i} F_{\phi}^{i}\left(m_{0}\right), \quad \boldsymbol{h} \in \mathbb{K}^{d} .
$$

Proposition A.10. Assume that the mixed derivatives $F_{\phi}^{i j} \doteq\left(F_{\phi}^{i}\right)_{\phi}^{j}$ and $F_{\phi}^{j i} \doteq\left(F_{\phi}^{j}\right)_{\phi}^{i}$, $i, j \in\{1, \ldots, d\}$ of the mapping $F: \mathcal{U} \rightarrow \mathfrak{V}$ exist and are continuous on $\mathcal{U}$. Then they coincide:

$$
F_{\phi}^{i j}\left(m_{0}\right)=F_{\phi}^{j i}\left(m_{0}\right), \quad m_{0} \in \mathcal{U}
$$

Proof. For $m_{0}=\phi^{-1}\left(\boldsymbol{t}_{0}\right) \in \mathcal{U}$ and sufficiently small $h, k \in \mathbb{K}$ consider the following expression which involves two increments of $F \circ \phi^{-1}$ :

$$
F \circ \phi^{-1}\left(\boldsymbol{t}_{0}+h \boldsymbol{e}_{j}+k \boldsymbol{e}_{i}\right)-F \circ \phi^{-1}\left(\boldsymbol{t}_{0}+h \boldsymbol{e}_{j}\right)-F \circ \phi^{-1}\left(\boldsymbol{t}_{0}+k \boldsymbol{e}_{i}\right)+F \circ \phi^{-1}\left(\boldsymbol{t}_{0}\right) .
$$

By assumption on the existence and continuity of the mixed derivatives we can apply the Mean Value Theorem A.7 twice to the above expression: One can consider the increments with respect to $\boldsymbol{e}_{i}$ and apply the Mean Value Theorem to them first and afterwards to the resulting integrand which takes on the form of an increment with respect to $\boldsymbol{e}_{j}$, or one carries out the same procedure with the rôles of $\boldsymbol{e}_{i}$ and $\boldsymbol{e}_{j}$ interchanged. Upon division by $h k \neq 0$ this yields the integrals

$$
\begin{aligned}
\int_{0}^{1} d \vartheta \int_{0}^{1} d \vartheta^{\prime} F_{\phi}^{i j} \circ \phi^{-1}\left(\boldsymbol{t}_{0}+\vartheta h \boldsymbol{e}_{j}+\vartheta^{\prime}\right. & \left.k \boldsymbol{e}_{i}\right), \\
& =\int_{0}^{1} d \vartheta \int_{0}^{1} d \vartheta^{\prime} F_{\phi}^{j i} \circ \phi^{-1}\left(\boldsymbol{t}_{0}+\vartheta h \boldsymbol{e}_{j}+\vartheta^{\prime} k \boldsymbol{e}_{i}\right),
\end{aligned}
$$

for any $h, k \in \mathbb{K} \backslash\{0\}$. Specializing to sequences $\left\{h_{n}\right\}_{n \in \mathbb{N}}$ and $\left\{k_{n}\right\}_{n \in \mathbb{N}}$ in this set which converge to 0 , it is a consequence of Lebesgue's Dominated Convergence Theorem (cf. [26, II.5.6 and II.6.2]) that for $n \rightarrow \infty$ the left-hand side converges to $F_{\phi}^{i j}\left(m_{0}\right)$ whereas the right-hand side approaches $F_{\phi}^{j i}\left(m_{0}\right)$ in the locally convex topology of $\mathfrak{V}$. Since this topology separates the elements of $\mathfrak{V}$, we conclude that these limits coincide and get the assertion by arbitrariness of $m_{0} \in \mathcal{U}$. 


\section{A.3 Differentiation on Automorphism Lie Groups}

The concepts developed thus far can now be applied to the case where the underlying analytic manifold is a (real or complex) Lie group $\mathcal{G}$ acting via a strongly continuous group of automorphisms $\left\{\alpha_{g}: g \in \mathcal{G}\right\} \subseteq$ Aut $\mathfrak{B}$ on the $C^{*}$-algebra $\mathfrak{B}$. These automorphisms $\alpha_{g}$, when applied to a given element $B \in \mathfrak{B}$, define a $\mathfrak{B}$-valued mapping on $\mathcal{G}$, for which statements can be proved that go beyond the above results. In doing so we shall be concerned with the canonical coordinates $\left(\mathcal{U}_{0}, \phi_{0}\right)$ of the first kind around the neutral element $\mathbf{1}$ of $\mathcal{G}$ where $\mathbf{1}=\phi_{0}^{-1}(\boldsymbol{0})$ (cf. [55, Section 2.10]). Note also, that, for given $g \in \mathcal{G}$, the left and right translations $l_{g}$ and $r_{g}$ on $\mathcal{G}$ as well as their composition $i_{g}=l_{g} \circ r_{g^{-1}}$ are analytic diffeomorphisms, so that their application to $\left(\mathcal{U}_{0}, \phi_{0}\right)$ yields local charts around $g$ and $\mathbf{1}$, respectively (cf. [55, Section 2.1]).

Proposition A.11. Let $\mathcal{G}$ be a d-dimensional real or complex Lie group and let $\mathfrak{B}$ be a $C^{*}$-algebra. For given $B \in \mathfrak{B}$ define the mapping

$$
\Xi_{B}: \mathcal{G} \rightarrow \mathfrak{B} \quad g \mapsto \Xi_{B}(g) \doteq \alpha_{g}(B) .
$$

(i) $\Xi_{B}$ is continuously differentiable on $\mathcal{G}$ if and only if it is differentiable at $\mathbf{1} \in \mathcal{G}$.

(ii) If $\Xi_{B}$ is differentiable at $\mathbf{1} \in \mathcal{G}$, then $\Xi_{\alpha_{g^{\prime}}(B)}$ is differentiable for any $g^{\prime} \in \mathcal{G}$ and the mapping

$$
\mathcal{G} \times \mathcal{U} \ni\left(g^{\prime}, g\right) \mapsto \mathfrak{D}_{\phi} \Xi_{\alpha_{g^{\prime}}(B)}(g) \boldsymbol{h}
$$

is jointly continuous in $g^{\prime}$ and $g$ for any local chart $(\mathcal{U}, \phi)$ around $g$ and any $\boldsymbol{h} \in \mathbb{K}^{d}$.

Proof. (i) To prove the non-trivial part, suppose that $g \in \mathcal{G}$ is arbitrary but fixed. Then $\left(g \mathcal{U}_{0}, \phi_{g}\right), \phi_{g} \doteq \phi_{0} \circ l_{g^{-1}}$, is a local chart around $g$ with $\phi_{g}^{-1}=l_{g} \circ \phi_{0}^{-1}$. According to the definition of $\Xi_{B}$ we have

$$
\Xi_{B} \circ \phi_{g}^{-1}=\Xi_{B} \circ l_{g} \circ \phi_{0}^{-1}=\alpha_{g} \circ \Xi_{B} \circ \phi_{0}^{-1}
$$

and, since the automorphisms are norm-preserving, the assumed differentiability of the mapping $\Xi_{B} \circ \phi_{0}^{-1}$ at 0 carries over to $\Xi_{B} \circ \phi_{g}^{-1}$ which by Definition A.8 means that $\Xi_{B}$ is differentiable at $g=\phi_{g}^{-1}(\boldsymbol{0})$ :

$$
\mathfrak{D}_{\phi_{g}} \Xi_{B}(g)=\mathfrak{D}\left(\Xi_{B} \circ \phi_{g}^{-1}\right)(\boldsymbol{0})=\alpha_{g} \circ \mathfrak{D}\left(\Xi_{B} \circ \phi_{0}^{-1}\right)(\boldsymbol{0})=\alpha_{g} \circ \mathfrak{D}_{\phi_{0}} \Xi_{B}(\mathbf{1}) .
$$

In view of (A.11) this relation can be re-written with respect to an arbitrary local chart $(\mathcal{U}, \phi)$ on $\mathcal{G}$ containing $g$ :

$$
\mathfrak{D}_{\phi} \Xi_{B}(g)=\mathfrak{D}_{\phi_{g}} \Xi_{B}(g) \circ\left(\phi_{g} \circ \phi^{-1}\right)^{\prime}(\phi(g))=\alpha_{g} \circ \mathfrak{D}_{\phi_{0}} \Xi_{B}(\mathbf{1}) \circ \mathbf{M}^{\phi}(g),
$$

where the matrix elements of $\mathbf{M}^{\phi}(g) \doteq\left(\phi_{g} \circ \phi^{-1}\right)^{\prime}(\phi(g))$ are analytic in $g \in \mathcal{U}$. Since the automorphisms are norm-preserving and act stongly continuous on $\mathfrak{B}$, it is evident that application of the above operator to any vector $\boldsymbol{h} \in \mathbb{K}^{d}$ yields a continuous mapping on $\mathbb{K}^{d}$ to $\mathfrak{B}$, thus establishing continuous differentiability of $\Xi_{B}$ on $\mathcal{G}$ as stated. 
(ii) Let $g^{\prime} \in \mathcal{G}$ be arbitrary and consider the local chart $\left(\mathcal{U}_{0} g^{\prime}, \psi_{g^{\prime}}\right), \psi_{g^{\prime}} \doteq \phi_{0} \circ r_{g^{\prime}}$, around $g^{\prime}$ with inverse $\psi_{g^{\prime}}^{-1}=r_{g^{\prime}} \circ \phi_{0}^{-1}$. Then

$$
\Xi_{\alpha_{g^{\prime}}(B)} \circ \phi_{0}^{-1}=\Xi_{B} \circ r_{g^{\prime}} \circ \phi_{0}^{-1}=\Xi_{B} \circ \psi_{g^{\prime}}^{-1},
$$

so that the assumed differentiablity of $\Xi_{B}$ at $\mathbf{1}$ and thus, according to the first part, at $g^{\prime}$ with respect to the local chart $\left(\mathcal{U}_{0} g^{\prime}, \Psi_{g^{\prime}}\right)$ implies differentiability of $\Xi_{\alpha_{g^{\prime}}(B)}$ at $\mathbf{1}$. By an application of (A.15) we have

$$
\mathfrak{D}_{\phi_{0}} \Xi_{\alpha_{g^{\prime}}(B)}(\mathbf{1})=\mathfrak{D}_{\Psi_{g^{\prime}}} \Xi_{B}\left(g^{\prime}\right)=\alpha_{g^{\prime}} \circ \mathfrak{D}_{\phi_{0}} \Xi_{B}(\mathbf{1}) \circ \mathbf{N}\left(g^{\prime}\right),
$$

where the matrix elements of $\mathbf{N}\left(g^{\prime}\right) \doteq\left(\phi_{g} \circ \psi_{g^{\prime}}^{-1}\right)^{\prime}(\boldsymbol{0})$ are analytic in $g^{\prime}$. This in turn can, again by use of (A.15), be generalized to any $g \in \mathcal{G}$ lying in the local chart $(\mathcal{U}, \phi)$ :

$$
\mathfrak{D}_{\phi} \Xi_{\alpha_{g^{\prime}}(B)}(g)=\alpha_{g} \circ \mathfrak{D}_{\phi_{0}} \Xi_{\alpha_{g^{\prime}}(B)}(\mathbf{1}) \circ \mathbf{M}^{\phi}(g)=\alpha_{g g^{\prime}} \circ \mathfrak{D}_{\phi_{0}} \Xi_{B}(\mathbf{1}) \circ \mathbf{N}\left(g^{\prime}\right) \circ \mathbf{M}^{\phi}(g),
$$

an expression which is obviously continuous in both variables $g^{\prime}$ and $g$ when applied to an arbitrary element $\boldsymbol{h}$ of $\mathbb{K}^{d}$.

Remark. Note, that in the case of differentiability of $\Xi_{B}$ the mapping $g \mapsto \mathfrak{D}_{\phi} \Xi_{B}(g)$ need not be continuous in the operator-norm topology of the Banach space of linear operators on $\mathbb{K}^{d}$ to $\mathfrak{B}$, since the automorphism group $\left\{\alpha_{g}: g \in \mathcal{G}\right\} \subseteq$ Aut $\mathfrak{B}$ is only supposed to be strongly continuous.

Consider those operators $B \in \mathfrak{B}$ for which the mapping $\Xi_{B}$ is continuously differentiable on $\mathcal{G}$. According to Proposition A.11 this is equivalent to differentiability at $\mathbf{1}$ with respect to the canonical coordinates $\left(\mathcal{U}_{0}, \phi_{0}\right)$. Therefore one can define mappings $\delta^{i}$ corresponding to the partial derivatives of $\Xi_{B}$ at $\mathbf{1}$ (cf. (A.12)) by

$$
\delta^{i}(B) \doteq \mathfrak{D}_{\phi_{0}} \Xi_{B}(\mathbf{1}) \boldsymbol{e}_{i}, \quad i=1, \ldots, d .
$$

Since $\Xi_{B}$ depends linearly on $B$, it is easily seen that

$$
\begin{aligned}
\delta^{i}\left(B_{1}+B_{2}\right) & =\delta^{i}\left(B_{1}\right)+\delta^{i}\left(B_{2}\right), \\
\delta^{i}\left(\lambda B_{1}\right) & =\lambda \delta^{i}\left(B_{1}\right),
\end{aligned}
$$

for any $\lambda \in \mathbb{K}$ and $B_{1}, B_{2}$ in $\mathfrak{B}$ subject to Proposition A.11. Moreover, $\Xi_{B_{1} B_{2}}=\Xi_{B_{1}} \Xi_{B_{2}}$, so that Proposition A.3 yields

$$
\delta^{i}\left(B_{1} B_{2}\right)=\delta^{i}\left(B_{1}\right) B_{2}+B_{1} \delta^{i}\left(B_{2}\right) .
$$

Equations (A.18a) through (A.18c) show that the mappings $\delta^{i}$ act as derivations of the $C^{*}$-algebra $\mathfrak{B}$ (cf. [24, Chapter III.9] and [48, Section 8.6]). Their domains are certain subalgebras which are invariant under transformations from the automorphism group $\left\{\alpha_{g}: g \in \mathcal{G}\right\}$, since by (A.16) for any $g^{\prime} \in \mathcal{G}$ and any $B \in \mathfrak{B}$ with differentiable $\Xi_{B}$ one has

$$
\delta^{i}\left(\alpha_{g^{\prime}}(B)\right)=\mathfrak{D}_{\phi_{0}} \Xi_{\alpha_{g^{\prime}}(B)}(\mathbf{1}) \boldsymbol{e}_{i}=\alpha_{g^{\prime}}\left(\mathfrak{D}_{\phi_{0}} \Xi_{B}(\mathbf{1}) \mathbf{N}\left(g^{\prime}\right) \boldsymbol{e}_{i}\right)=\sum_{j=1}^{d} \mathbf{N}_{j i}\left(g^{\prime}\right) \alpha_{g^{\prime}}\left(\delta^{j}(B)\right) .
$$


Let $\mathrm{l}_{M}$ denote the $M$-tuple $\left(i_{1}, \ldots, i_{M}\right)$ with integer entries $1 \leqslant i_{l} \leqslant d$, then the corresponding products of derivations $\delta^{i_{M}} \doteq \delta^{i_{M}} \ldots \delta^{i_{1}}$ act as linear operators on certain subspaces of $\mathfrak{B}$ which are again invariant with respect to $\left\{\alpha_{g}: g \in \mathcal{G}\right\}$, possibly the trivial space $\{0\}$ (note, that in general the derivations will not commute). Making use of the concepts of differentiability introduced above together with the fact that left and right translations act as analytic diffeomorphisms on the group $\mathcal{G}$, it is a matter of elementary considerations to establish the following connection between products $\delta^{{ }_{M}}$ of the above kind and the partial derivatives of the mapping $\Xi_{B}$ indexed by multi-indices $\kappa:$

$$
\begin{aligned}
& \Xi_{B, \phi}^{\kappa}(g)=\sum_{\mathfrak{l}_{M}, M \leqslant|\kappa|} C_{\kappa, l_{M}}^{\phi}(g) \alpha_{g}\left(\delta^{\mathfrak{l}_{M}}(B)\right), \\
& \delta^{\mathrm{l}_{M}}(B)=\sum_{\kappa,|\kappa| \leqslant M} D_{\mathrm{l}_{M}, \boldsymbol{\kappa}}^{\phi_{0}}(\mathbf{1}) \Xi_{B, \phi_{0}}^{\kappa}(\mathbf{1}) .
\end{aligned}
$$

Here the real or complex functions $C_{\mathrm{\kappa}, l_{M}}^{\phi}$ and $D_{\mathrm{l}_{M}, \mathrm{\kappa}}^{\phi_{0}}$ are analytic on the respective charts $(\mathcal{U}, \phi)$ and $\left(\mathcal{U}_{0}, \phi_{0}\right)$, containing $g$ and $\mathbf{1}$ respectively. Implicit in (A.20a) and (A.20b) is the perception that the mapping $\Xi_{B}$ is $N$-fold (or infinitely often) continuously differentiable if and only if the operator $B$ belongs to the domain of all $\delta^{L^{M}}$ for $M \leqslant N$ (or any $M<\infty)$.

We formulate these results in the following definition and subsequent proposition.

Definition A.12. Let $\delta^{i}, i=1, \ldots, d$, denote the partial derivations pertaining to the mappings $\mathcal{G} \ni g \mapsto \Xi_{B}(g)=\alpha_{g}(B) \in \mathfrak{B}$ for certain $B \in \mathfrak{B}$ via

$$
\delta^{i}(B) \doteq \mathfrak{D}_{\phi_{0}} \Xi_{B}(\mathbf{1}) \boldsymbol{e}_{i}
$$

For given $N \in \mathbb{N}$ the domain of arbitrary $N$-fold products $\delta^{l_{N}}$ of these derivations is an invariant subspace of $\mathfrak{B}$ with respect to the automorphism group $\left\{\alpha_{g}: g \in \mathcal{G}\right\}$ and denoted $\mathcal{D}^{(N)}(\mathfrak{B})$ : the space of $N$-fold differentiable operators. The elements of the space $\mathcal{D}^{(\infty)}(\mathfrak{B}) \doteq \bigcap_{N \in \mathbb{N}} \mathcal{D}^{(N)}(\mathfrak{B})$ in turn are called infinitely often differentiable with respect to $\left\{\alpha_{g}: g \in \mathcal{G}\right\}$. Accordingly, the resulting operators $\delta^{\boldsymbol{l}_{N}}(B)$ are designated as the derivatives of $B \in \mathfrak{B}$, if this element happens to lie in their domain.

Proposition A.13. For given $B \in \mathfrak{B}$ the mapping

$$
\Xi_{B}: \mathcal{G} \rightarrow \mathfrak{B} \quad g \mapsto \Xi_{B}(g) \doteq \alpha_{g}(B)
$$

is $N$-fold or infinitely often continuously differentiable if and only if the operator $B$ belongs to $\mathcal{D}^{(N)}(\mathfrak{B})$ respectively $\mathcal{D}^{(\infty)}(\mathfrak{B})$.

\section{A.4 Differentiable Linear Mappings}

In this section a special notion of differentiability for linear mappings on a locally convex space $\mathfrak{V}$ is introduced, which is motivated by the following result that is valid under the assumption of continuity. 
Proposition A.14. Let $\mathfrak{X}$ be a (real or complex) normed space, $\mathfrak{G} \subseteq \mathfrak{X}$ open, and let $\mathfrak{V}$ and $\mathfrak{W}$ be locally convex spaces over the same field $\mathbb{K} \doteq \mathbb{R}$ or $\mathbb{K} \doteq \mathbb{C}$ with topologies defined by the families $\left\{q_{\lambda}: \lambda \in L\right\}$ and $\left\{q_{\mu}^{\prime}: \mu \in M\right\}$ of seminorms separating the points of $\mathfrak{V}$ and $\mathfrak{W}$, respectively. If $F: \mathfrak{G} \rightarrow \mathfrak{V}$ is differentiable at the point $\mathfrak{x} \in \mathfrak{G}$ and $\Psi: \mathfrak{V} \rightarrow \mathfrak{W}$ is a continuous linear mapping then the composition

$$
\Psi \circ F: \mathfrak{G} \rightarrow \mathfrak{W}
$$

is differentiable at $\mathfrak{x}$, too, and its derivative is given by

$$
\mathfrak{D}(\Psi \circ F)(\mathfrak{x})=\Psi \circ \mathfrak{D} F(\mathfrak{x}) .
$$

If $F$ is differentiable on all of $\mathfrak{G}$ the same holds true for $\Psi \circ F$ and (A.22) is valid for any $\mathfrak{x} \in \mathfrak{G}$.

Proof. By assumption on $F$ (relations (A.1a) and (A.1b)) in connection with linearity of $\Psi$, the increment of $\Psi \circ F$ at $\mathfrak{x}$ allows for the representation

$$
(\Psi \circ F)(\mathfrak{x}+\mathfrak{h})-(\Psi \circ F)(\mathfrak{x})=\Psi \circ \mathfrak{D} F(\mathfrak{x}) \mathfrak{h}+\Psi(R[F, \mathfrak{x}](\mathfrak{h})),
$$

where

$$
\lim _{\mathfrak{h} \rightarrow 0}\|\mathfrak{h}\|^{-1} q_{\lambda}(R[F, \mathfrak{x}](\mathfrak{h}))=0
$$

for any seminorm $q_{\lambda}, \lambda \in L$. But, due to continuity of $\Psi$, there exist to any seminorm $q_{\mu}^{\prime}$ on $\mathfrak{W}$ a finite number of seminorms $q \lambda_{i}$ on $\mathfrak{V}, i=1, \ldots, N$, and a positive constant $C_{\mu}$ such that for any $\mathfrak{v} \in \mathfrak{V}$

$$
q_{\mu}^{\prime}(\Psi(\mathfrak{v})) \leqslant C_{\mu} \max _{1 \leqslant i \leqslant N} q_{\lambda_{i}}(\mathfrak{v}),
$$

and therefore

$$
0 \leqslant\|\mathfrak{h}\|^{-1} q_{\mu}^{\prime}(\Psi(R[F, \mathfrak{x}](\mathfrak{h}))) \leqslant C_{\mu} \max _{1 \leqslant i \leqslant N}\left(\|\mathfrak{h}\|^{-1} q_{\lambda_{i}}(R[F, \mathfrak{x}](\mathfrak{h}))\right) \underset{\mathfrak{h} \rightarrow \mathfrak{o}}{\longrightarrow} 0 .
$$

This is just the formulation of (A.1b) for $\Psi \circ F$ and thus proves, according to (A.23), differentiability of this mapping at $\mathfrak{x}$ together with (A.22). The remainder of the assertion is a trivial consequence.

The above results can easily be generalized to $\mathfrak{V}$-valued mappings on an analytic manifold $\mathcal{M}$.

Corollary A.15. Let $\mathcal{M}$ be a (real or complex) analytic manifold of dimension $d$ and let $\mathfrak{V}$ and $\mathfrak{W}$ be locally convex spaces over the same field. If $F: \mathcal{U} \rightarrow \mathfrak{V}$ is differentiable at the point $m_{0} \in \mathcal{U},(\mathcal{U}, \phi)$ a local chart on $\mathcal{M}$, and $\Psi: \mathfrak{V} \rightarrow \mathfrak{W}$ is a continuous linear mapping then

$$
\Psi \circ F: \mathcal{U} \rightarrow \mathfrak{W}
$$

is differentiable at $m_{0}$, and its derivative is given by

$$
\mathfrak{D}_{\phi}(\Psi \circ F)\left(m_{0}\right)=\Psi \circ \mathfrak{D}_{\phi} F\left(m_{0}\right) .
$$

Accordingly, $\Psi \circ F$ is differentiable on all of $\mathcal{M}$ in case that $F$ is. 
Proposition A.14 motivates the following definition which does no longer depend on the assumption of continuity.

Definition A.16. (a) Let $\mathfrak{X}$ be a normed space and $\mathcal{F}$ a family of differentiable mappings on $\mathfrak{X}$ with values in a locally convex space $\mathfrak{V}$. A linear mapping $\Psi$ on $\mathfrak{V}$ to the locally convex space $\mathfrak{W}$ is called $\mathcal{F}$-differentiable if and only if $\Psi \circ F: \mathfrak{X} \rightarrow \mathfrak{W}$ is differentiable on $\mathfrak{X}$ for any $F \in \mathcal{F}$ with

$$
\mathfrak{D}(\Psi \circ F)(\mathfrak{x})=\Psi \circ \mathfrak{D} F(\mathfrak{x}), \quad \mathfrak{x} \in \mathfrak{X} .
$$

(b) Let $\mathcal{M}$ be an analytic manifold and let $\mathfrak{V}, \mathfrak{W}$ and $\Psi$ be as above. Assume furthermore that $\mathcal{F}$ is a family of differentiable $\mathfrak{V}$-valued mappings on $\mathcal{X}$. Then $\Psi$ is called $\mathcal{F}$-differentiable if and only if $\Psi \circ F: \mathcal{M} \rightarrow \mathfrak{W}$ is differentiable on $\mathcal{M}$ for any $F \in \mathcal{F}$ and

$$
\mathfrak{D}_{\phi}(\Psi \circ F)\left(m_{0}\right)=\Psi \circ \mathfrak{D}_{\phi} F\left(m_{0}\right)
$$

for any chart $(\mathcal{U}, \phi)$ around the arbitrary element $m_{0} \in \mathcal{M}$. 


\section{Appendix B}

\section{A Lemma on Norm-Separable $C^{*}$-Algebras}

The following result is an adaptation of [43, Lemma 14.1.17] to our needs.

Lemma B.1. Let $\mathfrak{A}$ be a unital $C^{*}$-subalgebra of $\mathfrak{B}(\mathcal{H})$, where the Hilbert space $\mathcal{H}$ is separable. There exists a norm-separable $C^{*}$-algebra $\mathfrak{A}^{0}$, containing the unit element $\mathbf{1}$, that lies strongly dense in $\mathfrak{A}$.

Proof. Let $\left\{\phi_{n}\right\}_{n \in \mathbb{N}}$ be a dense sequence of non-zero vectors in $\mathcal{H}$ and let $\mathfrak{M} \doteq \mathfrak{A}^{\prime \prime}$ denote the von Neumann algebra generated by $\mathfrak{A}$. According to von Neumann's Density Theorem, $\mathfrak{M}$ coincides with the strong closure $\mathfrak{A}^{-}$of the algebra $\mathfrak{A}$, which by assumption acts non-degenerately on $\mathcal{H}$ (cf. [24, Section I.3.4], [11, Corollary 2.4.15]).

First we assume the existence of a separating vector for $\mathfrak{M}$, which is thus cyclic for $\mathfrak{M}^{\prime}$ [24, Section I.1.4]. Then any normal functional on $\mathfrak{M}$ is of the form $\omega_{\psi, \psi^{\prime}}\lceil\mathfrak{M}$ with $\psi, \psi^{\prime} \in \mathcal{H}\left[54\right.$, Theorem V.3.15]. Choose operators $A_{j, k} \in \mathfrak{A}_{1}$ satisfying

$$
\omega_{\phi_{j}, \phi_{k}}\left(A_{j, k}\right) \geqslant \| \omega_{\phi_{j}, \phi_{k}}\left\lceil\mathfrak{M} \|-2^{-1},\right.
$$

which is possible due to Kaplansky's Density Theorem [48, Theorem 2.3.3]. Let $\mathfrak{A}^{0}$ denote the norm-separable $C^{*}$-algebra generated by the unit element 1 together with all the operators $A_{j, k}, j, k \in \mathbb{N}$, and select a normal functional $\omega_{\xi, \theta}$ on $\mathfrak{M}$ with the properties $\| \omega_{\xi, \theta}\left\lceil\mathfrak{A}^{0} \|=0\right.$ and $\| \omega_{\xi, \theta}\lceil\mathfrak{M} \|>0$. Without loss of generality we can assume $\| \omega_{\xi, \theta}\left\lceil\mathfrak{M} \|=1\right.$. To any $\varepsilon>0$ there exist vectors $\phi_{j}, \phi_{k}$ from the dense sequence in $\mathcal{H}$ rendering $\left\|\phi_{j}-\xi\right\|$ and $\left\|\phi_{k}-\theta\right\|$ small enough so that

$$
\|\left(\omega_{\xi, \theta}-\omega_{\phi_{j}, \phi_{k}}\right)\lceil\mathfrak{M} \|<\varepsilon .
$$

Making use of (B.1) we then get the estimate

$$
\begin{aligned}
\varepsilon>\|\left(\omega_{\xi, \theta}-\omega_{\phi_{j}, \phi_{k}}\right)\left\lceil\mathfrak{M}\|\geqslant\|\left(\omega_{\xi, \theta}-\omega_{\phi_{j}, \phi_{k}}\right)\left(A_{j, k}\right) \|\right. \\
\quad=\left\|\omega_{\phi_{j}, \phi_{k}}\left(A_{j, k}\right)\right\| \geqslant \| \omega_{\phi_{j}, \phi_{k}}\left\lceil\mathfrak{M} \|-2^{-1},\right.
\end{aligned}
$$

which in connection with (B.2) implies

$$
\| \omega_{\xi, \theta}\left\lceil\mathfrak { M } \| \leqslant \| ( \omega _ { \xi , \theta } - \omega _ { \phi _ { j } , \phi _ { k } } ) \left\lceil\mathfrak { M } \| + \| \omega _ { \phi _ { j } , \phi _ { k } } \left\lceil\mathfrak{M} \|<2 \varepsilon+2^{-1} .\right.\right.\right.
$$


By arbitraryness of $\varepsilon$ we infer $\| \omega_{\xi, \theta}\left\lceil\mathfrak{M} \| \leqslant 2^{-1}\right.$ in contradiction to the assumption that $\omega_{\xi, \theta}\left\lceil\mathfrak{M}\right.$ be normalized. Thus, $\omega_{\xi, \theta}\left\lceil\mathfrak{A}^{0}=0\right.$ implies $\omega_{\xi, \theta}\lceil\mathfrak{M}=0$, i. e. any normal functional on $\mathfrak{M}$ annihilating $\mathfrak{A}^{0}$ annihilates $\mathfrak{M}$ as well. Now, since the $C^{*}$ algebra $\mathfrak{A}^{0}$ acts non-degenerately on $\mathcal{H}$, von Neumann's Density Theorem tells us that its strong and $\sigma$-weak closures coincide with $\mathfrak{A}^{0^{\prime \prime}}=\mathfrak{A}^{0^{-}}$, and this in turn is equal to the von Neumann algebra $\mathfrak{M}$; for the existence of an element $A \in \mathfrak{M}$ not contained in $\mathfrak{A}^{0^{-}}$would, by the Hahn-Banach-Theorem, imply a $\sigma$-weakly continuous (normal) functional that vanishes on $\mathfrak{A}^{0}$ but not on $A \in \mathfrak{M} \backslash \mathfrak{A}^{0^{-}}$in contradiction to the above result.

Now suppose that there does not exist a separating vector for the von Neumann algebra $\mathfrak{M}=\mathfrak{A}^{-}$. Then the sequence

$$
\left(\left(n\left\|\phi_{n}\right\|\right)^{-1} \phi_{n}\right)_{n \in \mathbb{N}} \subseteq \underline{\mathcal{H}} \doteq \bigoplus_{n=1}^{\infty} \mathcal{H}
$$

is such a vector for the von Neumann algebra $\mathfrak{M} \doteq\left(\bigoplus_{n=1}^{\infty} \mathfrak{l}\right)(\mathfrak{M})$, where $\mathfrak{l}$ denotes the identity representation of $\mathfrak{M}$ in $\mathcal{H}$. The result of the preceding paragraph thus applies to the $C^{*}$-algebra $\underline{A} \doteq\left(\bigoplus_{n=1}^{\infty} \mathfrak{l}\right)(\mathfrak{A})$ of operators on the separable Hilbert space $\underline{\mathcal{H}}$ which is weakly dense in $\underline{\mathfrak{M}}: \underline{\mathfrak{A}}^{-}=\underline{\mathfrak{M}}$. We infer that there exists a norm-separable $C^{*}$-subalgebra $\underline{\mathfrak{A}}^{0}$ of $\underline{\mathfrak{A}}$ including its unit $\underline{\mathbf{1}} \doteq(\mathbf{1})_{n \in \mathbb{N}}$, which is strongly dense in $\underline{\mathfrak{A}}$. Now, $\underline{\mathfrak{l}} \doteq \bigoplus_{n=1}^{\infty} \mathrm{l}$ is a faithful representation of $\mathfrak{A}$ on $\underline{\mathcal{H}}$ and its inverse $\underline{\mathfrak{t}}^{-1}: \underline{\mathfrak{A}} \rightarrow \mathfrak{A}$ is a faithful representation of $\mathfrak{A}$ on $\mathcal{H}$ which is continuous with respect to the strong topologies of $\underline{\mathfrak{A}}$ and $\mathfrak{A}$. Therefore $\mathfrak{A}^{0} \doteq \underline{\mathfrak{t}}^{-1}\left(\underline{\mathfrak{A}}^{0}\right)$ is a norm-separable $C^{*}$-subalgebra of $\mathfrak{A}$, containing the unit element $\mathbf{1}$ and lying strongly dense in $\mathfrak{A}$. 


\section{Bibliography}

[1] Alfsen, E. M. Compact Convex Sets and Boundary Integrals. Springer-Verlag, Berlin, Heidelberg, New York, 1971.

[2] ArAKi, H. Einführung in die Axiomatische Quantenfeldtheorie I. Vorlesung, Eidgenössische Technische Hochschule, Zürich, Wintersemester 1961/62. Ausgearbeitet von K. Hepp und F. Riahi.

[3] Araki, H., And HaAg, R. Collision cross sections in terms of local observables. Commun. Math. Phys. 4 (1967), 77-91.

[4] ARveson, W. An Invitation to $C^{*}$-Algebras. Springer-Verlag, New York, Heidelberg, Berlin, 1976.

[5] Asimow, L., AND Ellis, A. J. Convexity Theory and its Applications in Functional Analysis. Academic Press, Inc., London, New York, 1980.

[6] BARUt, A. O., And RąCZKA, R. Theory of Group Representations and Applications, 2nd rev. ed. Państwowe Wydawnictwo Naukowe-Polish Scientific Publishers, Warszawa, 1980.

[7] Baumgärtel, H., And Wollenberg, M. Causal Nets of Operator Algebras. Akademie-Verlag, Berlin, 1992.

[8] Bishop, E., AND DE LeEuw, K. The representation of linear functionals by measures on sets of extreme points. Ann. Inst. Fourier (Grenoble) 9 (1959), 305331.

[9] Borchers, H.-J. Translation group and spectrum condition. Commun. Math. Phys. 96 (1984), 1-13.

[10] Borchers, H.-J., AND Buchholz, D. The energy-momentum spectrum in local field theories with broken Lorentz-symmetry. Commun. Math. Phys. 97 (1985), 169-185.

[11] Bratteli, O., And Robinson, D. W. Operator Algebras and Quantum Statistical Mechanics 1, 2nd ed. Springer-Verlag, New York, Berlin, Heidelberg, 1987.

[12] Bratteli, O., And Robinson, D. W. Operator Algebras and Quantum Statistical Mechanics 2, 2nd ed. Springer-Verlag, Berlin, Heidelberg, New York, 1997. 
[13] Buchiolz, D. Gauss' law and the infraparticle problem. Phys. Lett. B 174 (1986), 331-334.

[14] BuchHolz, D. On particles, infraparticles, and the problem of asymptotic completeness. In VIIIth International Congress on Mathematical Physics (Marseille, 1986), M. Mebkhout and R. Sénéor, Eds., World Scientific, pp. 381-389.

[15] BuchHolz, D. Harmonic analysis of local operators. Commun. Math. Phys. 129 (1990), 631-641.

[16] Buchholz, D., D’ Antoni, C., And Longo, R. Nuclear maps and modular structures II: Applications to quantum field theory. Commun. Math. Phys. 129 (1990), 115-138.

[17] Buchholz, D., And Porrmann, M. To be published.

[18] BuchHolz, D., AND Porrmann, M. How small is the phase space in quantum field theory? Ann. Inst. Henri Poincaré - Physique théorique 52 (1990), 237-257.

[19] Buchholz, D., Porrmann, M., And Stein, U. Dirac versus Wigner: Towards a universal particle concept in local quantum field theory. Phys. Lett. B 267 (1991), 377-381.

[20] Buchholz, D., AND Wichmann, E. H. Causal independence and the energylevel density of states in local quantum field theory. Commun. Math. Phys. 106 (1986), 321-344.

[21] Choquet, G. Existence et unicité des représentations intégrales au moyen des points extrémaux dans les cônes convexes. Séminaire BOURBAKI 9 (December 1956), 139-01-139-15.

[22] Courant, R., And John, F. Introduction to Calculus and Analysis - Volume II. John Wiley \& Sons, New York, London, Sydney, Toronto, 1974.

[23] DiRAC, P. A. M. The Principles of Quantum Mechanics, 4th ed. At The Clarendon Press, Oxford, 1958.

[24] Dixmier, J. Von Neumann Algebras. North-Holland Publishing Co., Amsterdam, New York, Oxford, 1981.

[25] Dixmier, J. C $C^{*}$-Algebras, rev. ed. North-Holland Publishing Co., Amsterdam, New York, Oxford, 1982.

[26] Fell, J. M. G., AND Doran, R. S. Representations of *-Algebras, Locally Compact Groups, and Banach *-Algebraic Bundles - Volume 1. Academic Press, Inc., San Diego, London, 1988.

[27] Fredenhagen, K., AND FREund, J. Work in progress.

[28] Fredenhagen, K., And Hertel, J. Zwei Sätze über Kompaktheit. Unpublished manuscript, 1979. 
[29] Fröhlich, J., Morchio, G., AND STrocchi, F. Charged sectors and scattering states in quantum electrodynamics. Ann. Phys. 119 (1979), 241-284.

[30] GRÜNING, A. Ladungssektoren positiver Energie im Hochenergielimes des Schwingermodells. Master's thesis, Universität Göttingen, 1999.

[31] HaAg, R. Local relativistic quantum physics. Physica 124A (1984), 357-364.

[32] HAAG, R. Local Quantum Physics, 2nd rev. and enl. ed. Springer-Verlag, Berlin, Heidelberg, New York, 1996.

[33] HAaG, R., AND KASTLER, D. An algebraic approach to quantum field theory. J. Math. Phys. 5 (1964), 848-861.

[34] HaAg, R., And Swieca, J. A. When does a quantum field theory describe particles? Commun. Math. Phys. 1 (1965), 308-320.

[35] Halmos, P. R. Measure Theory, 12th ed. D. Van Nostrand Company, Inc., Princeton, New Jersey, Toronto, Melbourne, London, 1968.

[36] Helgason, S. Groups and Geometric Analysis. Academic Press, Inc., Orlando, London, 1984.

[37] Heuser, H. Lehrbuch der Analysis - Teil 2, 8th ed. B. G. Teubner, Stuttgart, 1993.

[38] Hewitt, E., And Ross, K. A. Abstract Harmonic Analysis II. Springer-Verlag, Berlin, Heidelberg, New York, 1970.

[39] Hewitt, E., And Stromberg, K. Real and Abstract Analysis, 2nd ed. Springer-Verlag, Berlin, Heidelberg, New York, 1969.

[40] Jantscher, L. Distributionen. Walter de Gruyter, Berlin, New York, 1971.

[41] JARChOW, H. Locally Convex Spaces. B. G. Teubner, Stuttgart, 1981.

[42] Kadison, R. V., And Ringrose, J. R. Fundamentals of the Theory of Operator Algebras - Volume I. Academic Press, Inc., New York, London, 1983.

[43] Kadison, R. V., And Ringrose, J. R. Fundamentals of the Theory of Operator Algebras - Volume II. Academic Press, Inc., Orlando, London, 1986.

[44] Köthe, G. Topological Vector Spaces I, 2nd rev. ed. Springer-Verlag, Berlin, Heidelberg, New York, 1983.

[45] Nachbin, L. The Haar Integral. D. Van Nostrand Company, Inc., Princeton, New Jersey, Toronto, New York, London, 1965.

[46] Nussbaum, A. E. Reduction theory for unbounded closed operators in Hilbert space. Duke Math. J. 31 (1964), 33-44.

[47] Pedersen, G. K. Measure theory for $C^{*}$ algebras. Math. Scand. 19 (1966), 131-145. 
[48] Pedersen, G. K. C $C^{*}$-Algebras and their Automorphism Groups. Academic Press, Inc., London, New York, San Francisco, 1979.

[49] Peressini, A. L. Ordered Topological Vector Spaces. Harper \& Row Publishers, New York, Evanston, London, 1967.

[50] Phelps, R. R. Lectures on Choquet's Theorem. American Book, Van Nostrand, Reinhold, New York, Toronto, London, Melbourne, 1966.

[51] ReEH, H., AND Schlieder, S. Bemerkungen zur Unitäräquivalenz von Lorentzinvarianten Feldern. Nuovo Cimento 22 (1961), 1051-1068.

[52] SchmüDgen, K. Unbounded Operator Algebras and Representation Theory. Birkhäuser Verlag, Basel, Boston, Berlin, 1990.

[53] Schroer, B. Infrateilchen in der Quantenfeldtheorie. Fortschr. Phys. 11 (1963), $1-32$.

[54] TAKesaki, M. Theory of Operator Algebras I. Springer-Verlag, New York, Heidelberg, Berlin, 1979.

[55] Varadarajan, V. S. Lie Groups, Lie Algebras, and Their Representations, 1st Springer ed. Springer-Verlag, New York, Berlin, Heidelberg, Tokyo, 1984.

[56] Weidmann, J. Lineare Operatoren in Hilberträumen. B. G. Teubner, Stuttgart, 1976.

[57] Wigner, E. P. On unitary representations of the inhomogeneous Lorentz group. Ann. of Math. 40 (1939), 149-204. 


\section{Acknowledgements}

My sincere gratitude is due to Prof. Dr. Detlev Buchholz for his support and forbearance. I not only learned a lot from his views on theoretical physics and its relationship to mathematics, but his ideas also constituted the basis on which I could erect my contributions to the topic presented in this thesis.

I should furthermore like to thank Prof. Dr. Klaus Fredenhagen for his immediate readiness to write the additional report on this work.

Financial support by the Deutsche Forschungsgemeinschaft is gratefully acknowledged which I obtained from the Graduiertenkolleg at the II. Institut für Theoretische Physik of the University of Hamburg.

I want to thank AnnA for all her support and encouragement, and for unrepiningly sharing the burden of my strain in the final stages of this project.

Eventually, I want to express my deepest gratitude to my parents for their patience and confidence. I am afraid that I shall be unable to pass back even only part of what they have done for me. 
German translation of the quotation on page 53

\section{DIODOROS: Griechische Weltgeschichte IV, 59 (5)}

(nach der Übersetzung von Otto Veh)

Theseus beseitigte auch bei Eleusis den Kerkyon, der die Passanten zum Ringkampf veranlaßte und den, der unterlag, umbrachte. Sodann tötete er auch den Prokrustes, wie er hieß, der am sogenannten Korydallos in Attika hauste. Der nötigte die vorüberziehenden Wanderer, sich auf ein Bett niederzulegen und war einer zu lang, dann schlug er ihm die herausragenden Körperteile ab; denen aber, die kleiner waren, zog er die Füße in die Länge, weshalb er den Namen Prokrustes erhielt. 


\section{Lebenslauf}

Name Martin Porrmann

Geburtsdatum 25. Januar 1962

Geburtsort Wolfenbüttel, Niedersachsen

28. August 1968-19. Juli 1972 Besuch der Wilhelm-Raabe-Schule in Wolfenbüttel

31. August 1972-20. Mai 1981 Besuch des Theodor-Heuss-Gymnasiums in Wolfenbüttel

20. Mai 1981 Abitur

WiSe 1981/82-WiSe 1983/84 Grundstudium der Physik und Philosophie an der Freien Universität Berlin

9. Februar 1984 Vorprüfung zur Diplomprüfung in Physik

SoSe 1984-WiSe 1988/89 Hauptstudium der Physik an der Universität Hamburg

23. Januar 1989 Diplom-Physiker, Titel der Diplomarbeit: 'Ein verschärftes Nuklearitätskriterium in der lokalen Quantenfeldtheorie'

ab SoSe 1989 Arbeit an der hier vorgelegten Dissertation

1. Oktober 1989-30. September 1992 Wissenschaftlicher Mitarbeiter an der Universität Hamburg

ab SoSe 1997 Fortsetzung der Arbeit an der Georg-August-Universität zu Göttingen

1. April 1997-30. April 1998 Wissenschaftlicher Mitarbeiter an der Universität Göttingen 